Prepared for:

U.S. Department of Energy

Office of Health and Environmental Research Washington, D.C. 20545

Prepe red by:

A.V. Nero

A.J. Gadgil

W.W. Nazaroff

and

K.L. Revzan

Under Contract No. DE-AC03-76SF00098

\section{Indoor Radon and Decay Products: Concentrations, Causes, and Control Strategies}

November 1990

DOE/ER- - 0480P

DE91 004941

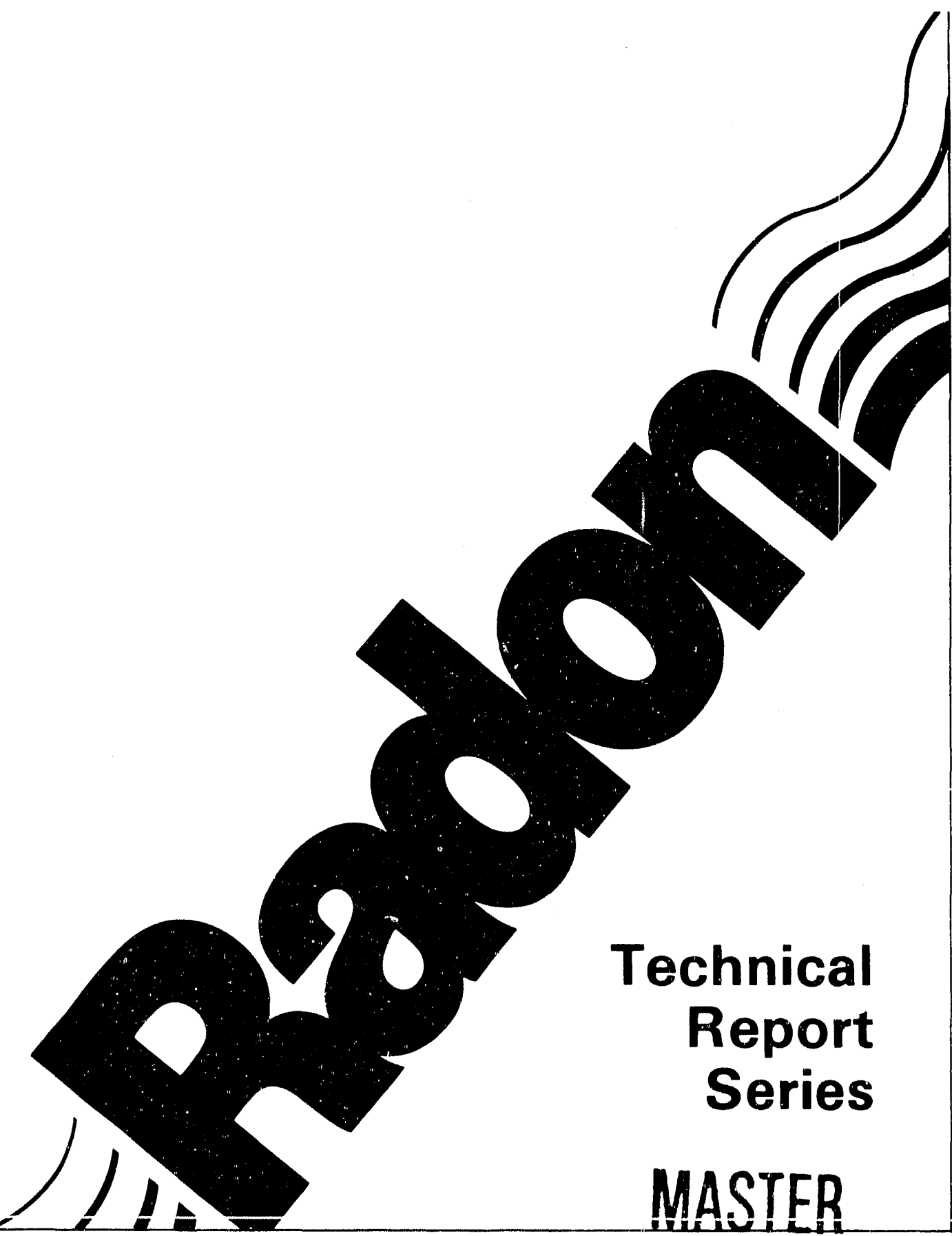




\section{Preface}

"Indoor Radon and Decay Products: Conrentrations, Causes, and Control Strategies," by Anthony V. Nero, Jr., Ashck J. Gadgil, William W. Nazaroff, and Kenneth L. Revzer is another in the ongoing technical report series that addresses various aspects of the DOE Radon Research Program. It provides an overview of what is known about the behavior of radon and its decay products in the indoor environment and examines the manner in which several important classes of factors - structural, geological, and meteorological - affect indoor radon concentrations. Information on U.S. indoor radon concentrations, currently available monitoring methods and novel radon control strategies are also explored.

The authors are members of the Indoor Environmental Program at Lawrence Berkeley Laboratory, which has for more than a decade studied the behavior and concentrations of radon in homes. Dr. Nero and his colleagues have in the past performed analyses and assessments of data and strategies on indoor radon that provide the basis for both the current radon research and radon stet policy. The authors are well known investigators and analysts in this field.

This report thus constitutes an important addition to the publications of the Radon Research Program of the Department of Energy's Office of Health and Environmental Research. Opinions and recommendations expressed in the text are those of tie authors. Information on the Radon Research Program and its publication may be obtained by writing to:

Susan L. Rose, Ph.D.

Radon Program Manager, ER-73

Office of Health and Environmental Research

Department of Energy, GTN

Washington, D.C. 20585 
Summary and Perspective on the Issues of Indoor Radon $\ldots \ldots \ldots \ldots \ldots \ldots \ldots$.

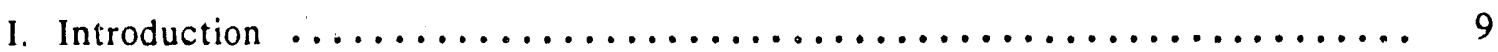

A. Historical perspective .............................. 9

B. Characteristics of radon and its decay products $\ldots \ldots \ldots \ldots \ldots \ldots \ldots, 13$

C. Behavior of radon indoors: general principles $\ldots \ldots \ldots \ldots \ldots \ldots \ldots \ldots$

II. Appraisal of concentrations in U.S. homes and other buildings ......... 19

A. Studies indicating the concentration distribution in U.S. housing ...... 21

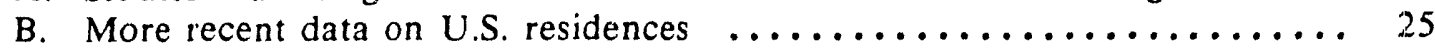

C. The occurrence of high concentrations or exposures $\ldots \ldots \ldots \ldots \ldots \ldots . \ldots 31$

D. Residential concentrations in other countries $\ldots \ldots \ldots \ldots \ldots \ldots \ldots \ldots \ldots . \ldots . \ldots$

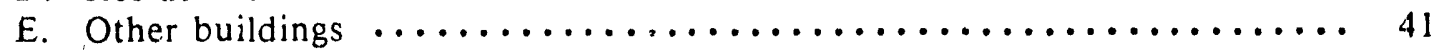

III. Factors affecting indoor concentrations $\ldots \ldots \ldots \ldots \ldots \ldots \ldots \ldots \ldots \ldots \ldots .42$

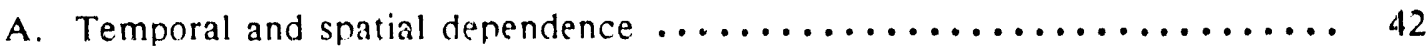

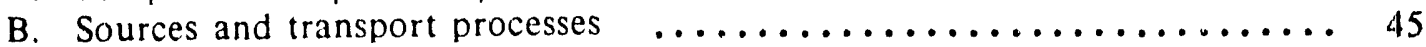

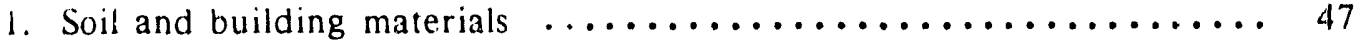

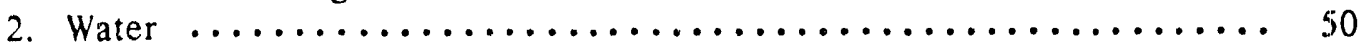

3. Summary of source contributions $\ldots \ldots \ldots \ldots \ldots \ldots \ldots \ldots \ldots \ldots \ldots$

C. Capabilities for predicting indoor concentrations $\ldots \ldots \ldots \ldots \ldots \ldots \ldots$. 51

1. House or site models ......................... 54

2. Predictive models for the housing stock $\ldots \ldots \ldots \ldots \ldots \ldots \ldots \ldots, 58$

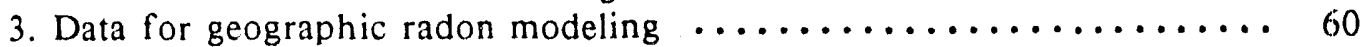

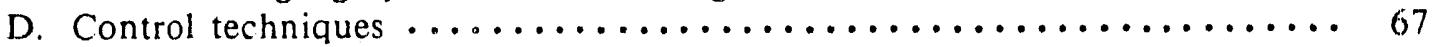

1. Techniques that reduce entry of radon from soil ............ 67

2. Detailed studies of radon entry and the influence of control techniques 68

3. Other types of control techniques $\ldots \ldots \ldots \ldots \ldots \ldots \ldots \ldots \ldots . \ldots 9$

IV. Techniques and protocols for monitoring indoor concentrations $\ldots \ldots \ldots \ldots \ldots$.

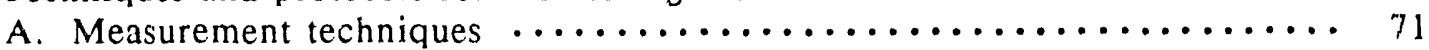

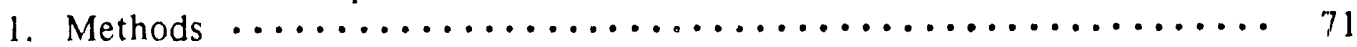

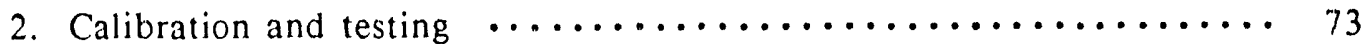

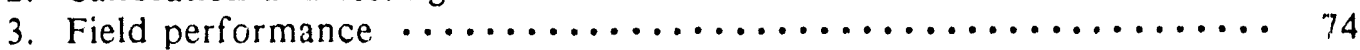

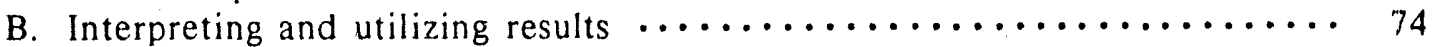

1. Techniques and strategies for identifying high-radon homes $\ldots \ldots \ldots 75$

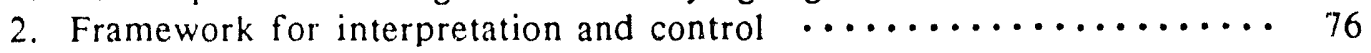

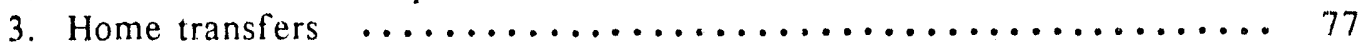

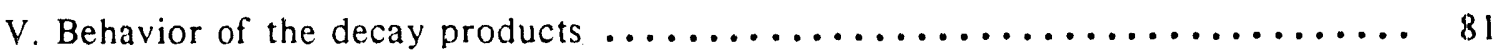

A. General considerations ................................ 81

B. The Jacobi model ................................ 82

C. Radioactive decay, recoil, and ventilation $\ldots \ldots \ldots \ldots \ldots \ldots \ldots \ldots \ldots \ldots$

D. Attachment ..................................... 85

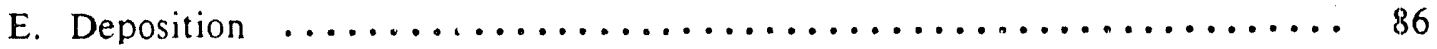

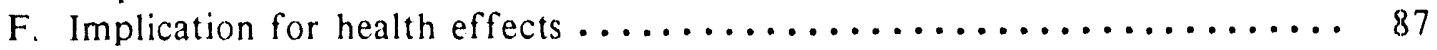

G. Effect of air-cleaning devices $\ldots \ldots \ldots \ldots \ldots \ldots \ldots \ldots \ldots \ldots \ldots \ldots \ldots$

H. Recent research on the nature and behavior of unattached ${ }^{218} \mathrm{P}_{0} \ldots \ldots .89$ 
VI. Strategies for controlling indoor concentrations $\ldots \ldots \ldots \ldots \ldots \ldots \ldots \ldots$. 91

A. Elements of control strategies $\ldots \ldots \ldots \ldots \ldots \ldots \ldots \ldots \ldots \ldots \ldots \ldots$ 91

1. Risk as a basis for specifying objectives ................. 94

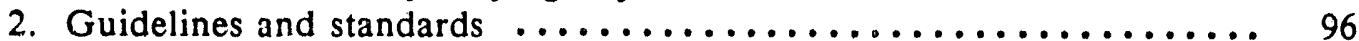

3. Identifying houses with excessive concentrations $\ldots \ldots \ldots \ldots \ldots . . . .97$

4. Selecting control techniques $\ldots \ldots \ldots \ldots \ldots \ldots \ldots \ldots \ldots \ldots . \ldots \ldots$. 99

B. Exploring policies for reducing exposures $\ldots \ldots \ldots \ldots \ldots \ldots \ldots \ldots \ldots, 102$

1. Form and implications of current policies $\ldots \ldots \ldots \ldots \ldots \ldots \ldots \ldots 102$

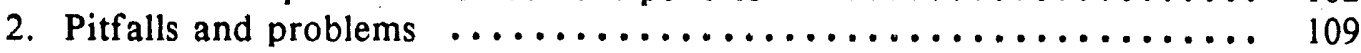

3. Moving towards a coherent policy $\ldots \ldots \ldots \ldots \ldots \ldots \ldots \ldots \ldots \ldots \ldots 11$

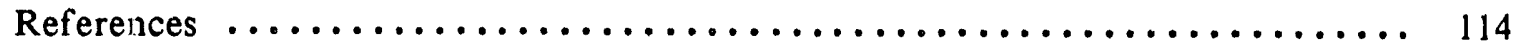

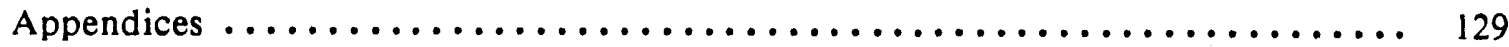

A. Radioactive quantities and units of measure $\ldots \ldots \ldots \ldots \ldots \ldots \ldots \ldots \ldots$

B. Ventilation rates: distribution and dependence $\ldots \ldots \ldots \ldots \ldots \ldots \ldots \ldots 131$

C. Outdoor concentrations of radon and its decay products $\ldots \ldots \ldots \ldots \ldots \ldots 133$

D. Radon 220 and its decay products $\ldots \ldots \ldots \ldots \ldots \ldots \ldots \ldots \ldots \ldots \ldots \ldots \ldots \ldots \ldots \ldots \ldots$ 
Since the mid-1980s, the importance of indoor radon as a potential health risk for the general public has finally come to be recognized. As a result, research efforts have become more extensive than they were before, and substantial efforts to reduce concentrations in U.S. homes have begun. This increased activity makes necessary a concerted effort to understand the growing amount of information available from research, monitoring, and control efforts and to assure that future research efforts and control strategies take proper account of this understanding. It is evident, however, from recent history that more effort must be devoted to utilizing, and more care to interpreting, the growing amount of information that is available.

The purpose of this report is to provide an introduction to the behavior of radon 222 and its decay products, based primarily on research of the last fifteen years, and to review certain important aspects of the radon question about which there has been significant misunderstanding. We therefore include 1) an appraisal of the concentration distribution in U.S. homes, 2) an examination of the utility and limitations of popular monitoring techniques and protocols, and 3) a discussion of the key elements of strategies for controlling radon levels in homes. It is useful here to summarize the main results from this review of radon and decay-product behavior, as well as to provide some perspective on the three key issues just mentioned.

Since 1980 , there have been approximately 15 international meetings devoted substantially or solely to the question of indoor radon, as well as many meetings focussing on narrow aspects of the question and numerous professional society meetings within the context of which indoor radon has been considered. In addition, hundreds of scientific articles on various aspects of indoor radon have appeared in a wide range of journals during the last dozen or so years. Such a wealth of meetings and articles reflects the substantial amount of research conducted since the mid-1970s on the occurrence, behavior, health effects, and control of radon and its decay products in the indoor environment. This research has already provided a reasonably comprehensive overall picture of indoor radon and has laid the groundwork for more effective identification of high-radon areas and houses and for more efficient reduction of indoor exposures where necessary.

Research on radon has included study of both the physical behavior and the biological effects of radon and its decay products. This report reviews only the former, but it is worth noting that research on the health effects of radon - including epidemiological studies of miner exposures and lung cancer, laboratory studies of animals, and theoretical studies of how radon's decay products flow into and are deposited in the lung - present a more or less convergent picture of the health risks associated with decay-product exposures. We are therefore in a reasonable position to make estimates of the risk from indoor exposures.

Thus, living for a lifetime at radon concentrations of, say, $740 \mathrm{~Bq} / \mathrm{m}^{3}(20 \mathrm{pCi} / \mathrm{l})$ causes doses in the range that apparently increased the risk of lung cancer among miners, causing an added chance of contracting this disease of 5 or $10 \%$. Living a lifetime in a $150 \mathrm{~Bq} / \mathrm{m}^{3}(4 \mathrm{pCi} / 1)$ house constitutes a proportionately smaller estimated risk, say 1 or $2 \%$. This is in the range of risks that we accept routinely from accidents in our homes or cars, but substantially greater than those from pollutants that are regulated in outdoor environmental media (air and water). Furthermore, these risks are the average of the estimates for smokers and nonsmokers, while in fact the preponderance of evidence suggests that the risks for smokers are significantly larger, and those for nonsmokers significantly smaller, than the percentages cited.

A difficulty, in any case, with estimating risks at radon concentrations of $150 \mathrm{~Bq} / \mathrm{m}^{3}$ and lower is that the epidemiological data do not provide direct evidence of health effects at the moderate exposures involved, in particular among the general public. On the other hand, the current understanding of dose-response relationships, especially for the alpha radiation from radon decay products, suggests the prudent course of presuming that the proportionately smaller lung 
cancer risks estimated for these exposures actually occur. A complication is that, based on epidemiological evidence, it is thought that the risk from radon is, to a greater or lesser degree, synergistic with that of smoking, so that most of the risk estimated for radon exposures of the general public occurs among smokers, who even without radon incur much greater risks (a $25 \%$ or greater chance of dying from smoking a pack and a half of cigarettes per day) knowingly and voluntarily. (It is thus ironic that exposures at $150 \mathrm{~Bq} / \mathrm{m}^{3}$ are of ten said to be equivalent in risk to smoking half a pack of cigarettes per day, a factor-of-ten overestimate for those who don't smoke.) Still, health risk estimates provide one of the main bases for determining whit radon concentrations might be deemed excessive and hence for focussing research and control efforts.

\section{Radon behavior}

Although it has long been known that radon occurs in all atmospheres, indoors and outdoors, a key initial finding of researchers was that indoor levels are considerably higher than those outdoors, simply because the radon enters into a much smaller atmosphere - i.e., the volume contained by the building interior. A primary result of early studies was that a very wide range of concentrations occurs indoors, ranging from a few $\mathrm{Bq} / \mathrm{m}^{3}$ (comparable to the typical outdoor level of $9 \mathrm{~Bq} / \mathrm{m}^{3}$, or $0.25 \mathrm{pCi} / \mathrm{l}$ ) to many thousands of $\mathrm{Bq} / \mathrm{m}^{3}$ (more than $100 \mathrm{pCi} / \mathrm{l}$ ). At about the same time it became clear that the major source of indoor radon was not usually the building materials or water supp'ies, such as had been studied intensively in Sweden and Maine, respectively, but the ground under the house, where radon is generated from radium that is naturally present in the soil. Such studies, completed by 1980 , led to experimental and theoretical research, as well as practical control programs (e.g., in Canada), that indicated that radon entered houses primarily by being carried in from the ground along with air that is drawn from the soil by small pressure differences between the house interior and the ground. These pressure differences are generated by the temperature differences that occur when indoor air is warmer than that outdoors, by wind-driven air flow around the structure, and - in some cases - by the building's heating or cooling system. It is these pressure differences that also cause general infiltration of air through the above-ground building shell, thus exchanging air between the indoor and outdoor atmospheres and providing the major means of ventilating small structures. This infiltration serves, then, to remove radon and other indoor-generated pollutants from the indoor atmosphere.

Although even now not understood in detail, this overall picture of radon production and entry provides a broad basis (1) for understanding the entry of radur into indoor environments, as well as the manner in which the wide range of indoor concentrations depends on soil, structural, and meteorological factors, and (2) for developing effective and efficient means for reducing entry where necessary. A principal result from the overall research on radon entry and removal is that whether or not a building has high or low indoor concentrations is determined primarily by the rate at which radon enters the structure, rather than by the ventilation rate. Programs to reduce energy use in buildings, for example by using weatherstripping and caulking, therefore appear to have had only a small effect on induor levels - to the point of being immeasurable in practice. Still, the same type of meteorological and structural factors affect both radon entry and energy losses through infiltration, and extreme forms of ventilation reduction certainly can affect indoor levels substantially. Research on radon entry, removal, and control thus needs to be conducted in the context of a full understanding of building design and operation. Hence the Department of Energy's support of research on indoor radon since the 1970s.

Experimental and theoretical studies of radon entry and removal thus provide an understanding of how indonr radon concentrations depend on the various classes of factors - whether geological, structural, or meteorological - that determine them. This understanding, together with calculational models and practical experience, can lead to more efficient identification of areas that tend to have high radon concentrations, as well as individual houses with excessive levels, and to better understanding and design of the control techniques that can be applied in various circumstances. 


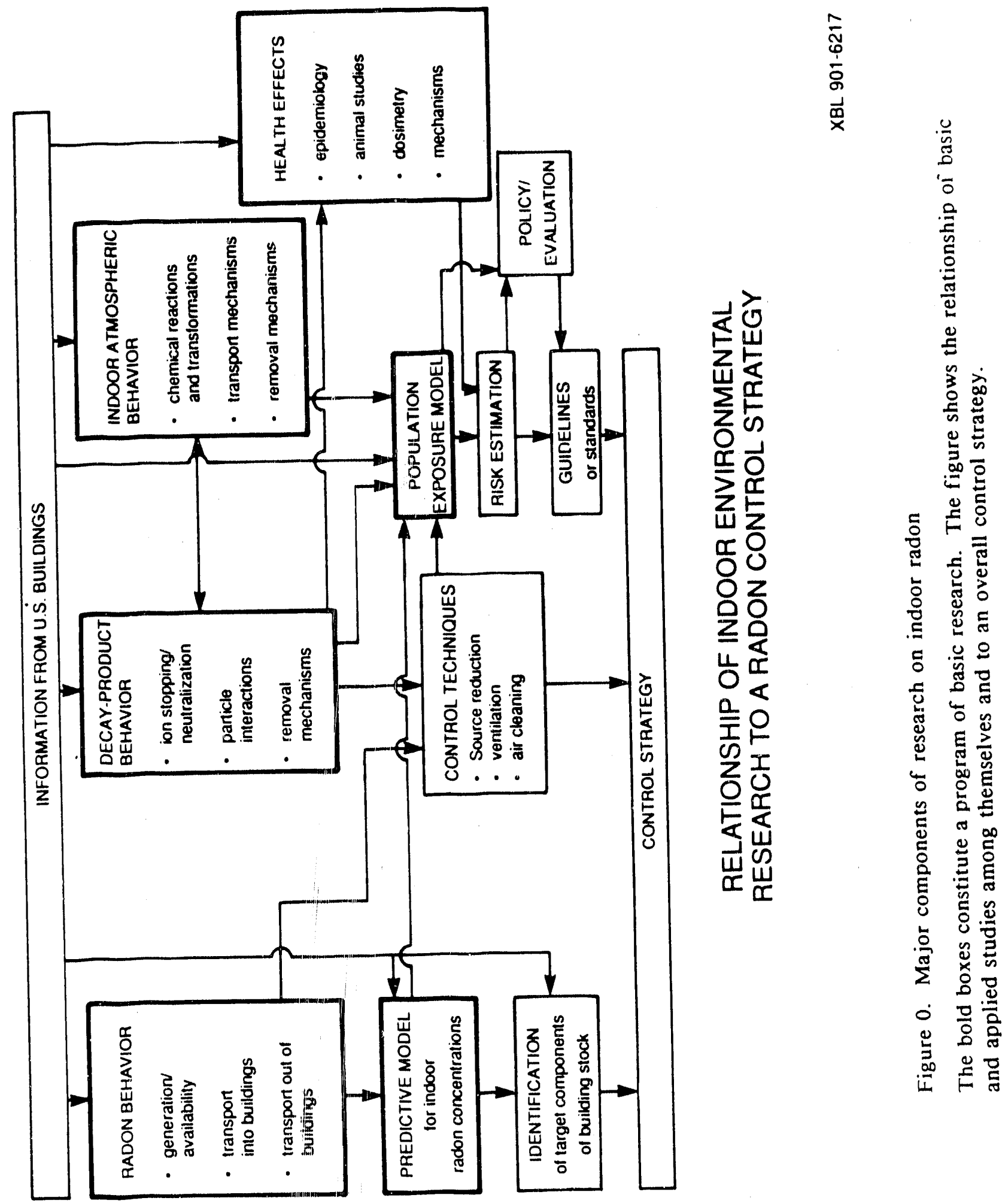


It is these two capabilities, identification and control, together with assessment of the health risks and a perspective on acceptable risks, that constitute the major elements needed for formulating an effective overall strategy for control of indoor radon.

Thus far, a concerted effort has not been constituted to develop these essential elements systematically or to examine the underlying premises and objectives in controlling the risk from radon (or other indoor pollutants). Indeed, even for the more elementary tasks of properly interpreting monitoring data or adopting appropriate monitoring protocols, much of what we know from basic studies of radon behavior has not been utilized. Before examining these issues, we turn to research on the behavior of the decay products.

\section{Decay-product behavior}

Decades ago it was realized that it is the short-lived decay products of radon, rather than the radon itself, that can deposit in the lung, thereby exposing the lining of the tracheobronchial tract to alpha particles from decay of the polonium members of the radon decay chain. It is this exposure that is thought to lead to the development of the excess numbers of lung cancers experienced by underground miners. Key work of the last two decades has been to understand what happens to the decay products after they are formed in the indoor atmosphere, both in terms of their attachment to preexisting particles in the air and their deposition on walls $\mathrm{cr}$ removal by ventilation or air cleaners, and in terms of the degree to which the decay products in different forms, either attached or not, deposit on and irradiate the lung lining when breathed. Aspects of this work include 1) basic studies of how the decay products behave in the air, from the time of their formation from radioactive decay to their attachment, deposition, or removal, 2 ) associated studies of the effect of various removal techniques, including ventilation and air cleaning, on the airborne concentrations of decay products in various forms, and 3 ) experimental and theoretical studies of deposition of decay products in the lung and the associated exposure of cells in the lung lining that can be transformed to a cancerous state.

These efforts have led to a functionally useful theoretical description of the behavior of the decay products indoors, based on an understanding of the characteristics of the radon decay series and on experimental determination of concentration ratios in various environmental conditions. Although further development is still needed to take account of the detailed physical and chemical behavior of the decay products and of the influence of air movement and other indoor environmental conditions, this theoretical picture is sufficient to lead to several important conclusions. First, both the unattached decay products (thought now actually to be molecular agglomerates formed rapidly around the decay products after they appear in the air from radioactive decay) and the attached form (resulting from attachment of these agglomerates to particles in the air) are important in determining the dose to the lung. That is, both deposit to a significant degree onto the lining of the tracheobronchial tract.

Secondly, although it is the airborne concentration of decay products that causes the dose of concern, the radon concentration appears to provide an equally good measure of this dose. For example, decay-product concentrations are relatively low compared with radon when the concentration of airborne particles is low, since this leads to more rapid deposition of decay products on the room walls (because the unattached molecular agglomerates are more highly mobile). However, a higher dose to the lung (in particular, the bronchial epithelium) per unit activity can result because the unattached decay products that remain in the air deposit in the lung with higher efficiency than decay products at ached to particles. As a result, air-cleaning methods that remove particles and decay products from the air, while able to reduce the total decay product concentration substantially, appear to have relatively little effect on the dose to the lung. This approach is therefore not ordinarily recommended as a means of reducing indoor levels. Rather, the preferred method for control is to reduce the entry of the radon itself into the indon " environment. 
Finally, an understanding of decay-product characteristics is a principal basis for the theoretical models for deposition and dose in the lung, such as have been used to understand the potential effects of air cleaning just mentioned. These models also provide a primary basis for our present understanding that exposure to decay products in homes can lead to similar doses, and hence presumably the same size risks, as exposures that occurred in mines.

\section{Radon concentrations in homes}

A key issue, then, is what radon concentrations occur in homes. By 1980 , it was clear that most houses have concentrations of tens of $\mathrm{Bq} / \mathrm{m}^{3}$ (say, 0.2 to $2 \mathrm{pCi} / \mathrm{l}$ ), but that significant numbers have substantially higher levels, with some having even thousanas of $\mathrm{Bq} / \mathrm{m}^{3}$ (say, $100 \mathrm{pCi} / \mathrm{l}$ ). In fact, increasing amounts of data in the early 1980s suggested that hundreds of thousands of U.S. homes migh: have levels of hundreds of $\mathrm{Bq} / \mathrm{m}^{3}$ (i.e., $10 \mathrm{pCi} / 1$, give or take a factor of two or three). The occurrence of average and high indoor concentrations was clarified considerably as a result of a 1984 analysis of earlier data and a year-iong survey in physics faculty's housing that began that same year. Both of these studies yielded an average concentration, predominantly in single-family housing, of about $55 \mathrm{~Bq} / \mathrm{m}^{3}(1.5 \mathrm{pCi} / \mathrm{l})$ and about $6 \%$ of houses exceeding $150 \mathrm{~Bq} / \mathrm{m}^{3}$ $(4 \mathrm{pCi} / 1)$. The studies indicated that 1 or $2 \%$ of houses have levels greater than $300 \mathrm{~Bq} / \mathrm{m}^{3}(8$ $\mathrm{pCi} / \mathrm{l})$ and, if the results are extrapolated, suggested that perhaps $0.1 \%$ of houses have levels exceeding $740 \mathrm{~Bq} / \mathrm{m}^{3}(20 \mathrm{pCi} / 1)$, the level at which occupants receive annual exposures comparable to the limit for miners.

Since the discovery of levels up to tens of thousands of $\mathrm{Bq} / \mathrm{m}^{3}$ (i.e., more than $1000 \mathrm{pCi} / \mathrm{l}$ ) in eastern Pennsylvania in late 1984, a much larger number of homes have been monitored than previously. Unfortunately, the resulting data have of ten been published or publicized in a way that leads to an exaggerated picture of indoor radon concentrations, in particular of average concentrations and the fraction of houses having elevated levels. This has occurred, as discussed below, not because the monitoring techniques employed are unreliable in themselves, but because they have been employed in ways that do not indicate the concentrations that people are actually exposed to or their frequency distribution. Thus published accounts of recent large amounts of data suggest that the average cuncentration in houses is abou: $150 \mathrm{~Bq} / \mathrm{m}^{3}(4 \mathrm{pCi} / \mathrm{l})$, which is two or three times the actual average, that 20 or $30 \%$ of houses have levels greater than $150 \mathrm{~Bq} / \mathrm{m}^{3}$, about four times the actual percentage, and that $1 \%$ have levels greater than $740 \mathrm{~Bq} / \mathrm{m}^{3}(20 \mathrm{pCi} / 1)$, while the real percentage appears to be a factor of ten lower.

These accounts have led to a substantial distortion of the general perception of indoor radon levels. This, in turn, has led to action programs aimed at the bulk of U.S. homes, which - ironically - can delay the formulation of programs focussed on finding and fixing houses with genuinely high levels. One contributor to this exaggerated perception of the problem, and to the formulation of overreaching rather than focussed efforts, is a sorresponding exaggeration of the risks entailed from radon exposures and of their significance, a fundamental issue in formulating control strategies. However, the simple misinterpretation and misrepresentation of monitoring data can contribute substantially to distorting the objectives and design of strategies for controlling excessive levels. It may be hoped that results from a national survey now being conducted by the Environmental Protection Agency (EPA) will reduce this difficulty.

\section{Monitoring techniques, protocols, and interpretation}

Techniques suitable for large-scale monitoring efforts have been available for several years. It is important to employ these techniques in protocols that are suitable for the intended purpose and to interpret the results properly. A major difficulty in portrayal of the radon problem in recent years has been the use of monitoring protocols that are suitable, at best, for screening purposes. Often, though, the results are used as though they were an actual indicator of human 
exposures, for example, by comparing them with the remedial action guideline of $150 \mathrm{~Bq} / \mathrm{m}^{3}(4$ $\mathrm{pCi} / \mathrm{l})$ recommended by the EPA. However the presently recommended screening protocol is a short-term measurement, placed in the basement if there is one, while the guideline applies to the average concentration to which occupants are actually exposed throughout the year. Even the average concentration in basements approaches or exceeds the action guideline, numerically. But a house with $150 \mathrm{~Bq} / \mathrm{m}^{3}\left(4 \mathrm{pCi} / \mathrm{l}\right.$ ) in the basement is likely to have about $50 \mathrm{~Bq} / \mathrm{m}^{3}$ (about 1.5 $\mathrm{pCi} / \mathrm{l})$ as an average concentration in the primary living space, where the guideline normally applies. Such differences account for much of the great discrepancy noted above in estimates of the percentage of homes with concentrations greater than such levels of concern as $150 \mathrm{~Bq} / \mathrm{m}^{3}(4 \mathrm{pCi} / \mathrm{l})$ and $740 \mathrm{~Bq} / \mathrm{m}^{3}(20 \mathrm{pCi} / \mathrm{l})$. Avoiding such misinterpretation requires that such screening data not be used for assessing average exposures or for indicating the probability of high concentrations.

However, a more fundamental problem persists, i.e., that any short-term screening protocol ought to be altered either in where the detector is deployed or in interpretation of the results, or both. Since the concern about radon has to do with human exposures, the better place to deploy a detector is in the primary living space. As presently designed, the raw result from the screening protocol typically gives a substantial overestimate of the concentration for one particular class of houses, i.e., those having basements. Since this large overestimation does not occur for other classes of houses, a substantial distortion is introduced into the general screening methodology, one that artificially introduces a much higher proportion of houses with basements into the follow-up pool than of houses built with a crawl space or a slab-on-grade foundation. Even for the latter substructure types, because the screening protocol is usually specified as a short-term winter test, concentrations also tend to be overestimated, but to a degree that varies from one part of the country to another (depending on variations in winter to annual-average concentrations that depend on environmental and other factors).

The major part of these discrepancies can be reconciled by monitoring in primary living space. However, it is also necessary to develop simple calculational methods that take account of the house design and location, and of data from the geographic area where the monitoring is being performed, to convert the raw monitoring result to an estimate of the annual-average concentration to which the occupants are actually exposed. This estimate can then sensibly be compared with the remedial action guideline. In this way, occupants will not be frightened when in fact levels are low, and remedial efforts can be focussed (and money expended) on the houses that actually have elevated levels.

A particular difficulty is that of real estate transactions, where a decision about remedial action is of ten wanted in a very short time, much shorter than the year-long monitoring that ought ordinarily to be the basis for a final decision on such action. For the house transfer situation, an interpretive protocol such as that just mentioned is almost essential to avoid inappropriate efforts and fears in large numbers of homes. This interpretive model would be a simplified representation, expressed in a simple computer model, of the current understanding of how soil, structural, and meteorological factors (for the house and area being considered) would be expected, in view of local monitoring experience, to affect indoor concentrations. It would yield the proportion between the long-term living-space concentration and the result from the monitoring protocol being used. Converting from the raw result to at least an estimate of the real concentration could substantially improve how decisions are made in real estate transactions. The situation would also be improved substantially by a better understanding of what radon levels actually indicate a need for action, an understanding that underlies development of an overall control strategy. 
Elements of strategies for controlling indoor radon

A national strategy for controlling indoor radon levels entails three major elements, each a complex system in itself. These are 1) a system of guidelines for limiting indoor concentrations or for controlling the factors that affect indoor levels, 2) a methodology for finding buildings with high concentrations, presumably including means for identifying the geographic areas where high concentrations have a higher-than-average probability of occurring, and 3 ) a decision framework for choosing the measures that will be applied to individual structures or, for the case of buildings yet to be constructed, classes of structures. These three elements are closely linked and their details depend substantially both on the growing scientific understanding of radon behavior and, just as importantly, on how we decide to deal with the risks of indoor pollutants in general.

Our general perspective on indoor risks critically affects the risk-limiting objectives that are chosen for indoor radon and, hence, the concentration-limiting recommendations or other measures that might be undertaken to limit public exposures. However, the risks from radon and other indoor pollutants are different in size and nature than those we experience in other contexts, as a result of which simple comparisons can lead to anomalous and of ten counterproductive results. Thus risks of cancer estimated for indoor pollutants tend to be much greater than those estimated for pollutants in outdoor air and water supplies. This fact might suggest the need for urgent, large-scale efforts. On the other hand, indoor risks are comparable in size to risks encountered in occupational settings, are in the same range or lower than accident risks in our homes and cars, and are far lower than the risks associated with cigarette smoking - which complicates consideration of radon considerably, since most of the risk from radon is thought to occur in association with cigarette smoking.

An essential element in formulating risk-limiting objectives for radon and other indoor pollutants is therefore an examination of the premises for isk avoidance or acceptability in this and other settings. The issues of individual control, knowiledge, cost, and benefit differ in each of these settings, which accounts for the fact that risk limits are substantially different in different circumstances. Appreciation of such differences also accounts, in part, for the fact that radon limits for new and existing housing can differ substantially from each other and from one country to another. Finally, from a practical point of view, considering for the moment only concentration limits (rather than other means - such as building codes - that can be used to limit exposures), only modest changes in numerical guidelines can have substantial effects on the number of houses affected. A guideline of $150 \mathrm{~Bq} / \mathrm{m}^{3}$ affects about $6 \%$ of single family houses, but would have substantial local differences - for example, it appears likely that no more than 1 out of a hundred California homes exceed this guideline. On the other hand, a limit of $740 \mathrm{~Bq} / \mathrm{m}^{3}(20 \mathrm{pCi} / \mathrm{l})$ appears to affect only about $0.1 \%$ of single-family houses nationwide, and probably a negligible percentage in California.

As a result, the precise guideline and the geographic area in question can affect drastically the method for identifying homes exceeding the guideline. If an area has been found, using a proper monitoring protocol, to have $20 \%$ of homes exceeding the guideline, then a recommendation that every home be monitored may be appropriate. On the other hand, if the percentage exceeding the guideline appears to be only one or a few percent, then a combination of surveys and modeling to identify areas that have a proportionately higher percentage of high concentrations appears appropriate. Utilization of the understanding of factors affecting indoor concentrations and of improved monitorıng techniques and protncols can contribute substantially to such efforts. Once such areas, and individual houses having high concentrations, are identified, one can utilize an appropriate decision matrix for determining what technical measures to employ, how to perform follow-up measurements, etc. 
The critical dependence of the design and cost effectiveness of a radon control strategy on an overall risk perspective and on the resulting risk-limiting objectives and associated concentration limits suggests urgent attention to this question, as well as continued substantial efforts to improve our basic understanding of radon and decay product behavior. Given the information from miner studies, as well as some consideration of estimated risks and of how society and individuals consider risk, it appears that homes having concentrations exceeding $740 \mathrm{~Bq} / \mathrm{m}^{3}(20 \mathrm{pCi} / \mathrm{l})$ or so should be a primary focus of initial control efforts. Individuals in these homes suffer significant risks, the estimates of which are supported fairly directly by epidemiological and other health evidence. This effort would entail finding and fixing approximately 100,000 homes across the United States. While this would be a substantial undertaking, and indeed would constitute one of the nation's major environmental efforts, it would not significantly affect the average exposure to indoor radon. Its sole objective would be to prevent individuals from suffering excessive risks, i.e., risks that are larger than most of us would accept in many circumstances. In addition, it would provide information relevant to identifying houses with only moderately high concentrations, and to lowering them efficiently, and it would identify areas where radon-specific building codes might be formulated to avoid high concentrations in new houses.

Extending the remedial effort to all homes with levels greater than $150 \mathrm{~Bq} / \mathrm{m}^{3}(4 \mathrm{pCi} / \mathrm{l})$ expands the efforts approximately fifty-fold, i.e., to perhaps 4 million homes. If successful, such a large-scale effort would reduce the (estimated) radon-related risks of virtually all individuals even smokers - to below those we routinely accept, such as the $2 \%$ individual risk of dying in car accidents. In addition, it would reduce the total number of lung cancers estimated to be associated with indoor radon by about 10 or $20 \%$, affecting primarily smokers. The last qualification is important, since the total number of cancers avoided by this multi-billion dollar commitment would be about $1 \%$ of the mortality caused by smoking itself. In terms of cost effectiveness, the commitment per premature death avoided would be much less than that for many environmental efforts, but much greater than measures related to accidents (such as smoke detectors or seat belts), and of course very much greater than the cost to avoid a smoking-related death.

Finally, to achieve the reduction of indoor levels to outdoor levels of $10 \mathrm{~Bq} / \mathrm{m}^{3}$ or so $(0.2$ or $0.3 \mathrm{pCi} / 1$ ) would require, over the long term, very substantial financial commitments in every home in the country, totalling hundreds of billions of doilars. This would, in effect, be aimed at constructing an impermeable shield against radon from the earth (as well as from water and building materials). These large differences among several possible goals make clear the importance of a concerted and systematic approach to the design of a strategy for controlling radon, including consideration of the basic premises and objectives for limiting risk in the indoor environment. 


\section{INTRODUC'TION}

\section{A. Historical Perspective}

The radiation dose from inhaled decay products of radon 222 is the dominant component of natural radiation exposures of the general population. Monitoring in various countries yields average residentiai ${ }^{222} \mathrm{Rn}$ concentrations ranging from 10 to $100 \mathrm{~Bq} / \mathrm{m}^{3}(0.3-3 \mathrm{pCi} / \mathrm{l}){ }^{1}$ For a country such as the United States, with an average residential concentration of about $40 \mathrm{~Bq} / \mathrm{m}^{3}(1 \mathrm{pCi} / \mathrm{l})$, the average lifetime risk of lung cancer caused by exposure to radon decay products is estimated to be about $0.4 \%$, causing, then, on the order of 10,000 cases of lung cancer annually ainong the U.S. population of 245 million. (See, for example, Lubin and Boice 1989.) As illustrated in Figure 1 - showing data from the United States that is discussed in section II.A - this risk is more significant than that received on the average from all other natural radiation sources or fiom medical exposures. Further, the dose from radon's decay produsts exceeds by a factor of 10 to 100 the average doses from nuclear power or weapons testing.

Probably more important is the fact that in any country or region indoor levels a factor of 10 or more higher than the average sometimes occur. In fact, it has been the common experience of the radon research community since the late $1970 \mathrm{~s}$ that ${ }^{322} \mathrm{R} n$ concentrations in the range of 200 to $2000 \mathrm{~Bq} / \mathrm{m}^{3}(5-54 \mathrm{pCi} / \mathrm{l})$ are found with startling frequency. And, although it might be thought that the estimated lung cancer risk associated even with an ordinary concentration such as $40 \mathrm{~Bq} / \mathrm{m}^{3}$ is very large compared with many environmental insults of concern (Nero 1988), living for prolonged periods at concentrations above 200 $\mathrm{Bq} / \mathrm{m}^{3}$ leads to estimated individual lifetime risks exceeding 1 or $2 \%$. The highest values found - far greater $2000 \mathrm{~Bq} / \mathrm{m}^{3}$ - have risks even larger than those from cigarette smoking. However, unlike smokers, those living in unusually high radon concentrations are rarely aware of the large risks they may thereby be suffering. (An important quaification for these estimates of radon risk, though, is that they average the risk for smokers and nonsmokers, while the evidence suggests that the risk for smokers is higher than that given, and that for nonsmokers is lower. See NAS 1988.)

The principal basis for present concern about exposures to radon's decay products is the experience with lung cancer incidence among underground miners. High fatality rates observed among miners as early as the sixteenth century were only later ascribed to lung cancer. And it was only after recognition in the 1950s that excessive lung cancer rates were occurring among uranium miners in the United States and elsewhere that examination of exposure history versus incidence indicated a relationship between added risk of lung cancer and exposures to ${ }^{222} \mathrm{Rn}$ decay products. Broadly speaking, results from a number of studies conducted among various miner groups, uranium and otherwise, have shown roughly consistent results. In the meantime, substantial efforts have been devoted to lowering exposure rates. Nonetheless, even current occupational limits - while keeping exposures much lower than before controls were implemented - are high enough that exposure at the limit still entails a significant risk and, indeed, one that is larger than the risk associated with exposure at the occupational limit for most other hazardous substances in the workplace.

This paper uses SI units throughout, except in examination of certain of the U.S. concentration data (where the original data are given in traditional units and conversion is difficult or awkward). See Appendix A for a discussion of quantities ard units. 


\section{Comparison To Other Radiation Exposures}

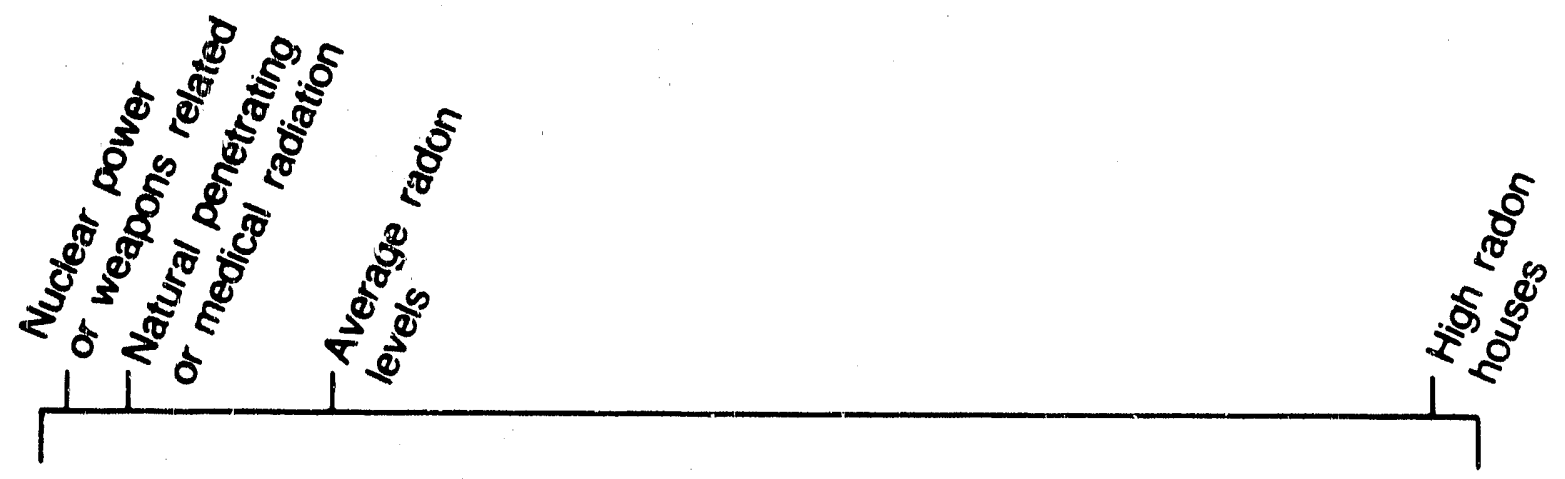

\section{${ }^{222} \mathrm{Rn}$ Concentration $\left(\mathrm{Bq} / \mathrm{m}^{3}\right)$}

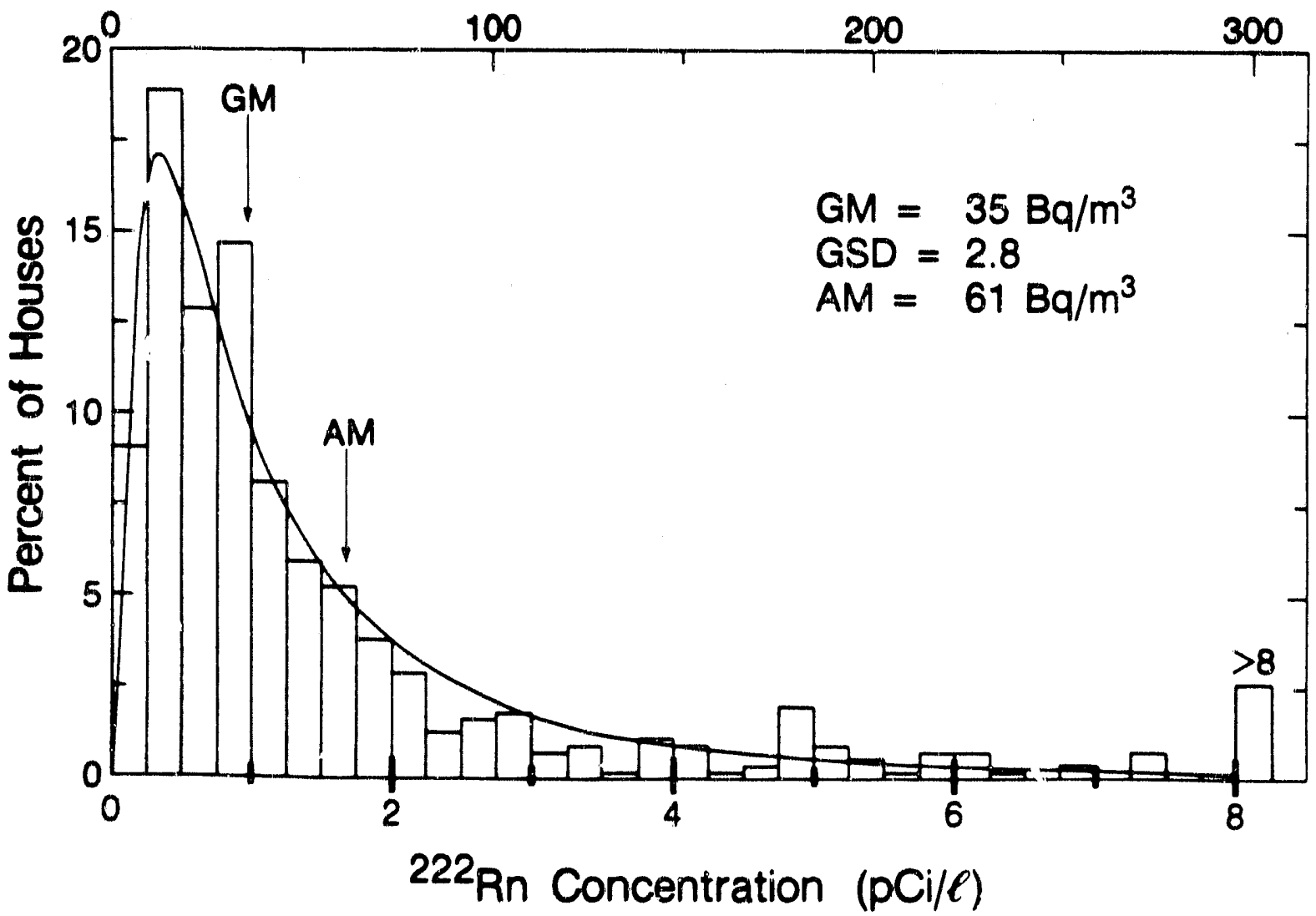

XBL $8411-4824 A$

Figure 1. Probability distribution of ${ }^{222} \mathrm{Rn}$ concentrations in U.S. homes

These data result from direct aggregation of 19 sets of data, totalling 552 homes, from the United States. The smooth curve is a lognormal function with the indicated parameters (Nero et al. 1986). The upper scale indicates approximately the relative effective doses from radon and other sources of radiation exposure. 
The experience gained in the uranium mines provided, not only information on health risks, but also the initial tools for understanding the occurrence, behavior, and control of radon and its decay products in the more general environment. The various isotopes of radon ${ }^{222} \mathrm{Rn}$ being the most important - are present to a greater or lesser degree in all environmental media - air, water, and soil - arising naturally from the radioactive decay of radium, whose isotopes are members of the decay series originating with uranium and thorium, primordial constituents of the earth's crust.

The initial focus of environmental studies, however, was still exposures resulting from industrial processes, primarily the mining and milling of uranium, that increased the accessibility of radon to the outdoor atmosphere or to indoor environments. A principal example has been high ${ }^{222} \mathrm{Rn}$ concentrations inside homes and other buildings in the vicinity of Grand Junction, Colorado, that were built on or with radium-rich tailings from uranium milling. Another case, in Canada, is that of the mining communities of Port Hope, Uranium City, Elliot Lake, and Bancroft, where remedial action programs were underiaken because of the possibility that indoor concentrations were unusually high because of local mining or milling activities. A comparable example is observation of higher-than-average indoor concentrations in homes built on lands in Florida that have been disturbed as the result of mining phosphate ores. These ores typically have elevated concentrations of the uraniurn series, and resulting waste products have high concentrations of radium. Although exposure limits were set by the United States and Canada for each of these cases, it is now clear that the concentrations found in these communities - while higher than average - are no higher than those occurring in many other areas due merely to radon from the ground or, in some cases, from building components using materials with natural concentrations of radionuclides.

Indication of the potential significance of radon in the general building stock came with the realization in the 1970s of the health implications of very high concentrations in Swedish homes built using light-weight concretes incorporating alum shale as aggregate. This shale had extremely high radium content, causing high radon emanation rates from the finished concrete. This, together with low ventilation rates prompted by the interest in reducing energy use, resulted in high airborne concentrations in this segment of the housing stock. Ironically, it has subsequently been found in Sweden that the bulk of radon in the total housing stock comes from the ground. Furthermore, monitoring in various countries has indicated that even average indoor concentrations are significant from the point of view of environmental risks. And, indeed, while changes in the ventilation rate can affect the indoor concentration significantly, the primary determinant of whether or not a particular indoor environment has high levels is, for many classes of buildings, the rate of radon entry.

Becausc of the apparent health implications, the early work on indoor concentrations has given rise to a broad range of research characterizing ${ }^{222} \mathrm{R} n$ and its decay products indoors. This work has included significant monitoring programs in homes, investigation of the sources of indoor radon, study of the behavior of the decay products, and development of techniaues for controlling indoor concentrations. In addition, radiobiologists and epidemiologists have begun to apply dosimetric and dose-response data explicitly to the problam of environmental exposures. These international efforts have resulted in an extensive literature, including the major collections indicated in Table 1 and hundreds of other papers that appear in a wide variety of journals and conference proceedings. 
Table 1

Major collections containing indoor radon articles

Natural Radiation Environment III, proceedings of Symposium, Houston, Texas, April 1978 (Edited by T. F. Gesell and W.M. Lowder) - Technical Information Center (CONF-780422), Springfield, Virginia, 1980.

Radon and Radon Daughters in Urban Communities Associated with Uranium Mining and Processing, proceedings of three AECB Workshops in Ontario, Canada, 1978-1980 - Atomic Energy Control Board, Ottay:/a Canada, 1978-1980.

Assessment of Radon and Daughter Exposure and Related Biological Effects, proceedings of Specialist Meeting, Rome, Italy, March 1980. (Edited by G.F. Clements, A.V. Nero, F. Steinhausler, M.E. Wrenn) - RD Press, Salt Lake City, Utah, 1982.

Natural Radiation Environment, proceedings of Second Special Symposium on Natural Radiation Environment, Bombay, India, January 1981. (Edited by K.G. Vohra, U.C. Mishra, K.C. Pillai, and.S. Sadasivan) - Wiley Eastern Limited, New Delhi, 1982.

Indoor Air Pollution, proceedings of the International Symposium on Indoor Air Pollution, Health and Energy Conservation, Amherat, Massachusetts, October 1981. (Edited by J. Spengler, C. Hollowell, D. Moschandreas, O. Y'snger) - Special Issue of Environment International 8 (Nos 1-6), 1982.

Indoor Radon (Edited by A.V. Nero and W.M. Lowder) - Special Issue of Health Physics 45 (No 2), August 1983.

Radon - Radon Progeny Measurements, proceedings of International Meeting, Montgomery, Alabama, August 1981 - U.S. Environmental Protection Agency (EPA 520/5-83/021), Washington, 1983.

Indoor Exposure to Natural Radiation and Associated Risk Assessment, proceedings of International Seminar, Anacapri, Italy, October 1983. (Edited by G.F. Clemente, H. Eriskat, M.C. O'Riordan, J. Sinnaeve) Special Issue of Radiation Protection Dosimetry 7 (Nos 1-4), 1984.

Exposure to Enhanced Natural Radiation and Its Regulatory Implications, proceedings of Seminar, Maastricht, Netherlands, March 1985. (Edited by B. Bosnjakovic, P.H. van Dijkum, M.C. O'Riordan, and J. Sinnaeve) - Special Issue of Science of the Total Environment 45, October 1985.

Indoor Radon, proceedings of APCA International Specialty Conference, Philadelphia, Pennsylvania, February 1986 - Air Pollution Control Association (SP-54), Pittsburgh, 1986.

Indoor Air Quality, based on the Third International Conference on Indoor Air Quality and Climate, Stockholm, August 1984. (Edited by B. Berglund, U. Bergland, T. Lindvall, J. Spengler, and J. Sundell) - Special Isoue of Environment International 12 (Nos. 1-4), 1986; more of the radon papers are in Radon, Passive Smoking, Particulates and Housing Epidemiology, Vol. 2 of Indoor Air (Edited by B. Berglund, T. Lindvall, and J. Sundell), Swedish Council for Building Research, Stockholm, 1984.

Radon and its Decay Products: Occurrence, Properties and Health Effects, proceedings of ACS Symposium, New York City, New York, April 1986. (Edited by P. Hopke) - American Chemical Society Symposium Series 331, Washington, 1987.

Indoor Radon II, proceedings of APCA International Specialty Conference, Cherry Hill, New Jersey, April 1987 Air Pollution Control Association (SP-60 and Supplement), Pittsburgh, 1987.

Indoor Air ' 87 proceedings of the Fourth International Conference on Indoor Air Quality and Climate, Vol. 2, Berlin, August 1987. (Edited by B. Seifert, H. Esdorn, M. Fischer, H. Ruden, and J. Wegner) - Institute for Water, Soll and Air Hygiene, Berlin, 1987; full papers to appear in special issue of Environment International.

Natural Radioactivity proceedings of the Fourth International Symposium on the Natural Radiation Environment, Lisbon, December 1987. (Edited by A.O. de Bettencourt, J.P. Galvao, W. Lowder, M. Olast, and J. Sinnaeve) - Special issue of R.adiation Protection Dosimetry 24 (nos 1-4), 1988.

Proceedings: the 1988 Symposium on Radon and Radon Reduction Technology, Denver, October 1988. Environmental Protection Agency Report EPA-600/9-89-006, Research Triangle Park, 1989

\section{Major Reviews}

Health Risk of Radon and Other Internally Deposited Alpha Emitters (Committee on the Biological Effects of lonizing Radiation) - National Academy Press, Washington, 1988.

Indoor Pollutants (Committee on Indoor Pollutants) - National Academy Press, Washingion, 1981.

Ionizing Radiation: Sources and Biological Effects (United Nations Scientific Committee on the Effects of Atornic Radiation) - United Nations, New York, 1982.

Evaluation of Occupational and Environmental Exposures to Radon and Radon Daughters in the United States Report No. 78, National Council on Radiation Protection and Measuremerits, Bethesda, MD., 1984.

Radon and Its Decay Products in Indoor Air (edited by W.W. Nazaroff and A.V. Nero)-Wiley, New York, 1988 
The present report serves as a broad introduction to our growing understanding of radon and its decay products in indoor air. It also reviews several critical issues related to exposure assessment and formulation of control straiegies. Specifically, we examine what we know about the concentrations and behavior of radon and its decay products, as weil as the utility of various monitoring techniques and the key elements of strategies for controlling indoor consentrations. A much more detailed review of the behavior (and health effects) of radon and its decay products is contained in Nazaroff and Nero (1988).

While the present paper serves as an introduction to the behavior of radon and its decay products, its utility lies equally in the examples it gives of important questions where the recent explosion of activity on radon has not been accompanied by corresponding care in the critical examination, interpretation, and evaluation of results. In our view, this difficulty has arisen partly from a lack of familiarity with the understanding that has arisen from the research of the las! decade, and partly from a tendency to interpret and act on the basis of preconceptions developed in other fields and contexts. The first causes repetition of past work or misinterpretation of current results, and the second leads to inappropriate and of ten counterproductive action. We hope the key issues we examine will illustrate the importance of unusual care in dealing with an exposure that causes, after all, risks that are estimated to be far larger than those often considered in "environmental" settings. This fact, taken alone, may lead to control programs that are more extensive and expensive than any previjus program of environmental control. But, in fact, control objectives and strategies should be devised also in the knowledge that these "large" risks estimated from indoor radon are, ironically, no larger than other risks that the public accepts daily as a matter of course. In general, the formulation of sensible strategies for dealing with indoor air quality requires development of a more complete perspective on environmental risk than has heretofore been available (Nero 1988).

\section{B. Characteristics of Radon and Its Decay Products}

A principal characteristic of radon that gives it more radiological significance than earlier members of the uranium (and thorium) decay chains is the fact that it is a noble gas. As such, once it is formed in radium-bearing material, a radon atom is relatively free to move, provided it first reaches the material's pore space (typically by recoil due to the parent radium atom's emission of an alpha particle). Once in the pore space, macroscopic transport of radon is possible, either by molecular diffusion or by flow of the fluid in the pore space. Radon can therefore reach air or water to which humans have access, provided that transport is sufficiently rapid to be completed before the radon decays.

Radon 222 - formed in the ${ }^{238} \mathrm{U}$ decay chain (Figure 2) from decay of ${ }^{226} \mathrm{Ra}$ - is the most important radon isotope because it has the longest half life, 3.8 days. This is long enough that much of the ${ }^{222} \mathrm{Rn}$ formed either in building materials or in the ground within approximately a meter of building understructures can reach the indoor environment. Similarly, much of the ${ }^{222} \mathrm{Rn}$ formed within about a meter of the earth's surface reaches the outdoor atmosphere, although this has less radiological significance than that reaching indoor environments, which have relatively small volumes compared with the contributing source material. In some cases, whether for the indoor or outdoor environment, radon from much larger distances than a meter can be important if high-permeability transport routes (such as gravelly soil or fissures in the ground) are available.

In contrast, although about as much ${ }^{220} \mathrm{Rn}$ (sometimes called thoron) activity is 'formed (in this case from decay of ${ }^{224} \mathrm{Ra}$, a member of the thorium 232 decay chain - see Appendix $D$ on $\left.{ }^{220} \mathrm{Rn}\right)$, substantially less reaches air because its short half life $(56 \mathrm{~s})$ limits the distance it 


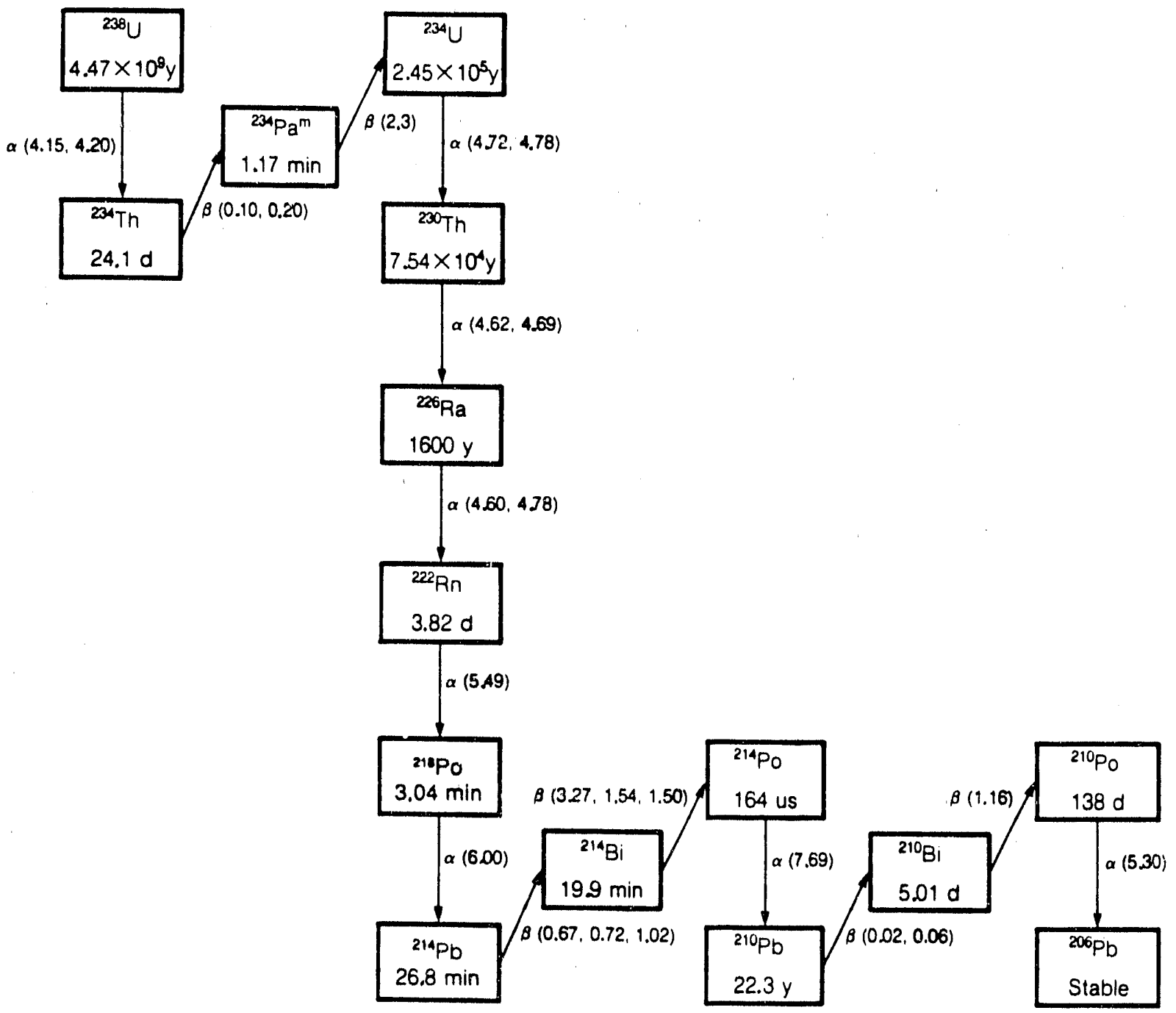

XBL 871.9206A

Figure 2. Uranium 238 decay chain, including ${ }^{222} \mathrm{Rn}$ and its decay products

Radon 222 , its parent ${ }^{226} \mathrm{Ra}$, and its decay products are members of the ${ }^{238} \mathrm{U}$ decay series. Airborne concentrations of ${ }^{218} \mathrm{Po},{ }^{214} \mathrm{~Pb}$, and ${ }^{214} \mathrm{Bi}$ are of prime radiological interest due to their potential for retention in the lung, leading to subsequent irradiation by the alpha decays of $218 \mathrm{Po}$ and $214 \mathrm{Po}$. Half-lives (in boxes) and alpha energies (in MeV) are from Browne and Firestone (1986), except for the ${ }^{218}$ Po half-life (from Martz et al. 1989). Beta end-point energies (in MeV) are from NCRP (1985), except for ${ }^{234} \mathrm{Th}$ and ${ }^{234} \mathrm{~Pa}^{\mathrm{m}}$, from Lederer and Shirley (1978). See Appendix D for comparable ${ }^{232} \mathrm{Th}$ decay chain (given in Figure 24). 
can travel before decay. ${ }^{2}$ Finally, very little of another radon isotope, ${ }^{219} \mathrm{Rn}$, is present in air because the ${ }^{235} \mathrm{U}$ decay chain, of which it is a member, has a natural abundance that is a factor of 100 lower and because of ${ }^{219} \mathrm{Rn}$ 's short half life $(4 \mathrm{~s})$.

The second important characteristic of radon is that it decays to radionuclides that are both chemically active and relatively short-lived. As indicated in Figure 2, the four radionuclides following decay of ${ }^{222} \mathrm{Rn}$ have half lives of less than 30 minutes, so that - if collected in the lung on being inhaled - they are likely to decay to ${ }^{210} \mathrm{~Pb}$ before removal by lung clearance mechanisms. (Similarly, ${ }^{220} \mathrm{Rn}$ begins a series of relatively short-lived isotopes; the most significant dose arises from the inhalation of ${ }^{212} \mathrm{~Pb}$, which has a half life of 10.6 h.)

The radiation released on decay of the short-lived decay products imparts the lung dose to which increased risk of lung cancer is attributer:. The alpha radiation from the polonium isotnpes contributes the radiologically significant dose, primarily because alpha particles deposit their energy within such a small thickness of tissue. As a result, the alpha energy is deposited in the relatively sensitive lung lining and also has a dense deposition pattern, which has much greater biological impact.

The concentration of (short-lived) decay products in air is not ordinarily given in terms of individual decay-product concentrations, but rather by a collective concentration that is normalized to the amount of alpha decay energy that will ultimately result from the mixture of decay products that is present. This quantity is the "equilibrium-equivalent" decayproduct concentration (EEDC), ${ }^{3}$ the amount of each decay product necessary to collectively have the same potential alpha energy concentration (PAEC) that is actually present. (The decay-product concentration can also be given in terms of the PAEC itself. See Appendix $A$ on quantities and units.) The ratio of EEDC to radon concentration is the equilibrium factor, equal to 1 if radon and all its decay products are in equilibrium (and therefore have the same radioactivity concentration), but in the range of $0.2-0.6$ for most indoor atmospheres and somewhat higher outdoors.

For a given indoor radon concentration, the concentrations of the decay products can vary over a substantial range, since they are removed from the air, not only by radioactive decay, but also by ventilation and by reactions with the structure and its furnishings. An additional and important manifestation of their chemical activity is the fact that the decay

Although indoor concentrations of the ${ }^{220} \mathrm{Rn}$ gas itself are ordinarily much less than those of ${ }^{222} \mathrm{Rn}$, scattered data (cf. Appendix on ${ }^{220} \mathrm{Rn}$ ) suggest that, at least in buildings with average or below-average ${ }^{222} \mathrm{Rn}$ concentrations, the potential alpha energy concentration (PAEC) of ${ }^{220} \mathrm{Rn}$ decay products - - while ordinarily less tan that from ${ }^{222} \mathrm{Rn}$ decay products - - can be a significant fraction of the total PAEC. Unfortunately, too little information is available to assess reliably the overall prevalence or importance of ${ }^{220} \mathrm{Rn}$ and its decay products, except to say that its PAEC has rarely, if ever, been found to approach the higher levels found for ${ }^{222} \mathrm{Rn}$ indoors. (And, in any case, the effective dose per unit PAEC is thought to be far smaller for the ${ }^{220} \mathrm{Rn}$ decay series than for the ${ }^{222} \mathrm{Rn}$ series; cf. James (1988).) As discussed later, however, results from measurements of indoor concentrations are consistent with a picture where ${ }^{222} \mathrm{Rn}$ transport is dominated by pressure driven flow, and ${ }^{220} \mathrm{Rn}$ transport by diffusion.

3 This quantity is of ten called the equilibrium-equivalent radon concentration, a practice that is avoided here because it is actually a measure of the decay-product concentration. 
products can form small airborne agglomerates and can attach to previously existing particles. Such characteristics of the airborne decay products affect the rate at which they deposit on the walls and furnishings, the pattern and degree of deposition in the lung, and ultimately - the magnitude and distribution of the associated radiation dose.

\section{Behavior of Radon Indoors: General Principles}

The indonr concentration of radon and its decay products, or of any other airborne pollutant, depends on three factors: the entry or production rate from various sources, the ventilation rate, and the rates of chemical or physical transformation or removal. Because of its relatively long half life and lack of chemical activity, ${ }^{222} \mathrm{Rn}$ itself acts much like a stable pollutant whose indoor concentration is determined by only two factors, the entry rate and the ventilation rate. In contrast, the behavior of the decay products is much more complex, deprnding on the radon that is present, the ventilation rate, and the interplay among radioactive decay, chemical reactivity, particle concentiations, and the nature of the boundary layer between the indoor atmosphere and the surfaces that enclose it. Nonetheless, as a practical matter, the decay-product concentration is indicated approximately by the radon concentration, which is determined by source and ventilation characteristics. The influence of these factors on indoor concentrations is discussed here briefly. In addition, Appendix B treats the question of ventilation rates per se.

In the observation of high concentrations in Swedish homes in the 1970s, both excessive entry rates and decreased ventilation rates appeared to be important contributors. These observations constituted a signal that scientists in other countries should investigate radon concentrations in their own building stocks. It was also in this same period that extensive research on other aspects of indoor air quality was beginning (see citations in Table 1), because of the discovery that other classes of pollution could occur indoors at higher levels than outdoors. For example, the byproducts of combustion - such as carbon monoxide, nitrogen oxides, and particulates - are the primary pollutants from the point of view of outdoor air quality. But concentrations are of ten much higher indoors, due to the presence of gas stoves, kerosene space heaters, and other appliances that are not vented effectively to the outdoors (Girman et al. 1982, Spengler and Sexton 1983). Similarly, although organic chemicals of various kinds are regulated in outdoor air and around toxic waste dumps, concentrations can be much higher indoors, again because of the presence of indoor sources (Miksch et al. 1982, McCann et al. 1987). In the United States - as well as in other countries - much of the research on indoor air quality began because of the fear that energy conservation measures might raise levels of such pollutants. This led, for example, to a substantial program of research supported by the U.S. Department of Energy (DOE), beginning in 1976.

The basis of this concern may be seen by considering the steady-state concentration of a nonreactive gas entering an interior space from effectively internal sources (including the ground) at a fixed rate per unit volume, $S_{V}$. The concentration in this case is easily shown to be

$$
I=\left(S_{V}+I_{o} \lambda_{v}\right) /\left(\lambda_{v}+d\right)
$$

where $\lambda_{v}$ is the ventilation rate, $I_{0}$ is the concentration in outdoor air, and $d$ is the decay rate of the gas (if applicable). For typical ventilation rates, greater than $0.1 \mathrm{~h}^{-1}$, the decay rate of ${ }^{222} \mathrm{Rn}\left(0.0076 \mathrm{~h}^{-1}\right)$ can be ignored in this equation, so that $1-I_{0}=S_{y} / \lambda_{v}$. This simplifies even further, to $I=S_{v} / \lambda_{v}$, when - as is often the case - the outdoor concentration is much smaller than the indoor. (Note that $\lambda_{v}$ and $d$ are rate constants, i.e., the fraction removed per unit time.) 
This expression does not encompass recently understood and important aspects of radon behavior, including time dependent and interactive phenomena, as discussed in later sections. Moreover, as is obvious, it cannot be used to describe the behavior of radon decay products, whose concentrations are determined by a number of interdependent reaction rates. Nonetheless, it is useful for specific purposes. An obvious example is that, for a fixed source strength, $S_{V}$, a decrease in the ventilation rate - e.g., to save energy will result in a corresponding increase in the indoor concentration.

However, the greater importance of source strengths in determining indoor radon concentraticns became clear in initial studies of indoor concentrations and ventilation rates. Earlier work had already indicated significant concentrations of ${ }^{222} \mathrm{R} n$ and its decay products in U.S. homes (George and Breslin 1980); and subsequent work had confirmed that, with supply of differing amounts of mechanical ventilation in a given house, the radon concentration varied as the inverse of the ventilation rate (Nazaroff et al. 1981). But results from simultaneous ${ }^{222} \mathrm{Rn}$ and ventilation-rate measurements in several groups of U.S. homes showed no apparent correlation between these two parameters (Nero et al. 1983). As shown in Figure 3, the radon concentrations and ventilations rates for each sample group showed an approximately order-of-magnitude range; for the combined sample of about 100 homes, the concentration showed a significantly larger variability than the ventilation rate. If the ventilation rate $\lambda_{v}$ and source strength $S_{v}$ were independent in this sample of houses, and if the range of concentrations observed were caused primarily by differences in ventilation rate, then - because $I=S_{y} / \lambda_{y}$ takes a linear form, $\ln I=\ln S-\ln \lambda_{y}$, on a logarithmic plot - the data points of Figure 3 would cluster around a straight line with slope -1 . No such correlation is apparent. The relative range and independence of these two quantities suggested that the source strength was the dominant determinant of the wide range of concentrations observed in U.S. housing.

Such indications prompted substantial work in understanding the size and variability of radon entry rates, as discussed below. However, it is important to emphasize that other factors still play an important role: ventilation rates vary substantially within the housing stock, which is of course one of the major incentives for past and current efforts to increase the efficiency of energy use in buildings. (In the United States, an added incentive is that even the average ventilation rate - see Appendix B - is relatively high compared with rates in many countries.) And, even for a given radon concentration, the concentrations and physical state of the decay products - which account for the health effects of principal concern - can vary significantly. We turn now to an appraisal oi concentrations in U.S. homes, and a brief review of concentrations elsewhere, af ter which we will examine more closely the factors affecting indoor levels. Concentrations of ${ }^{220} \mathrm{Rn}$ and its decay products are reviewed briefly in Appendix D. 


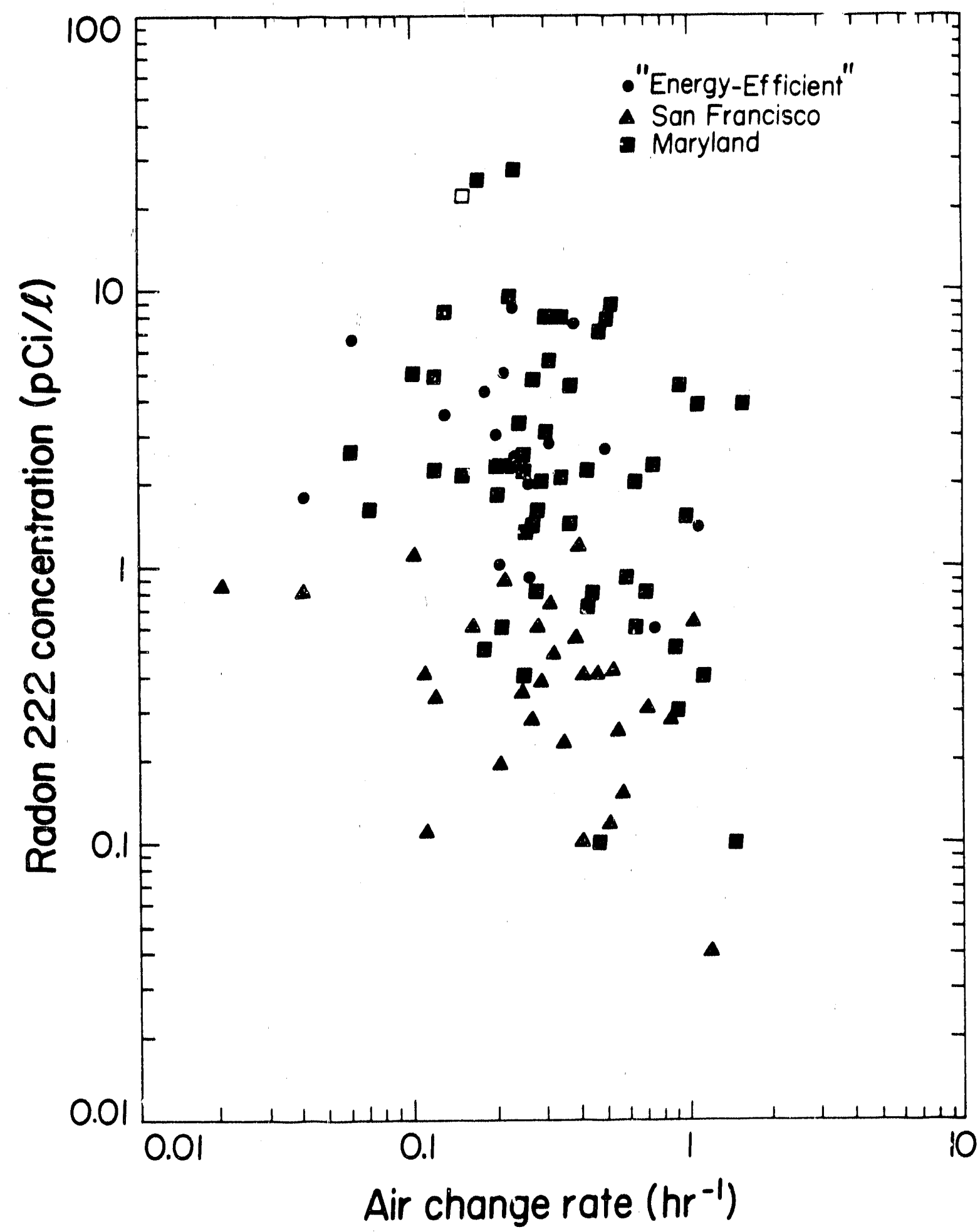

XEL $818-1115$

Figure 3. Radon 222 concentrations and air change rates measured in 98 U.S. residences

The results shown are from three survey groups: "energy-efficient" houses in the United States and (one) in Canada; conventional houses in the San Francisco area; and conventional houses in a community in rural Maryland (data from Nero et al. 1983). 


\section{APPRAISAL OF CONCENTRATIONS IN U.S. HOMES AND OTHER BUILDINGS}

Since identification of ${ }^{222} \mathrm{Rn}$ and its decay products as potentially important indoor pollutants, a large number of efforts have been undertaken actually measuring concentrations in homes. In a few countries, mostly European, these efforts have included surveys in statistically-designed samples of the housing stock. This has not yet occurred in the United States (though a national survey sponsored by the U.S. Environmental Protection Agency (EPA) is now under way). However, there have been many studies and much monitoring conducted in the United States, and - taken together - results to date provide a useful appreciation of residential radon concentrations even in the United States.

Interestingly, the most useful indication of the distribution of concentrations in the United States arises from studies undertaken prior to the explosion of activity since the discovery of extremely high indoor levels in eastern Pennsylvania and New Jersey. This is true because the earlier results offer tise possibility of estimating the annual-average concentration in living space without the problem of oversampling in areas known to have hign concentrations. This contrasts with the more than a million measurements performed since 1985, either taken in houses where the owners request monitoring (which tends to concentrate monitoring in locales known to have high radon levels indoors) or performed using short-term detectors, of ten placed in basements (which therefore does not indicate levels to which the occupants are typically exposed). Unfortunately, the public - and even many scientists - have been misled by the careless use of such data (which can, however, be useful for their own purposes).

Data are less complete for commercial and public buildings, although a variety of information can be brought to bear to suggest the approximate scale of radon concentrations in such environments.

This section briefly reviews the results of indoor radon surveys, primarily those performed in residences. No attempt is made to mention the many specific studies that have been performed. In particular, for residences - the focus of large numbers of studies - only the few surveys or analyses that are representative of a large segment of the housing stock are discussed explicitly. Results from a significant number of individual surveys may be found in the major references given in Table 1 and in numerous individual journal articles and reports. The studies indicative of concentrations in particular countries are summarized in Table 2.

It is also worth noting the tendency of the research community to measure radon concentrations in survey efforts, rather than decay-product concentrations. This tendency arises largely from the availability of reasonably reliable and very simple integrating etchedtrack radon monitors (see, for example, Alter and Fleischer 1981), a significant contrast to the state of decay-product monitoring. (An alternative technique being used in many shorter-term studies, and in screening measurements recommended by the EPA, is based on collection of radon for a few days in charcoal canisters, as discussed in Section IV; see, for example, Cohen and Cohen 1983.) Fortunately, given a reasonable understanding of the relationship between radon and its decay products and an awareness of the fact that the decay-product-to-radon ratio (typically in the range of 0.2 to 0.8 ) does not vary as widely as radon concentrations, measurement of the radon is a reasonable indicator of decayproduct concentrations and is a very effective tool in survey efforts. This is analogous to the situation for many other pollutants: for example, although hea!th effects associated with $\mathrm{NO}_{2}$ exposures may have a substantial dependence on peak (rather than average) concentrations, an integrating sampler can be a very effective survey instrument, provided 


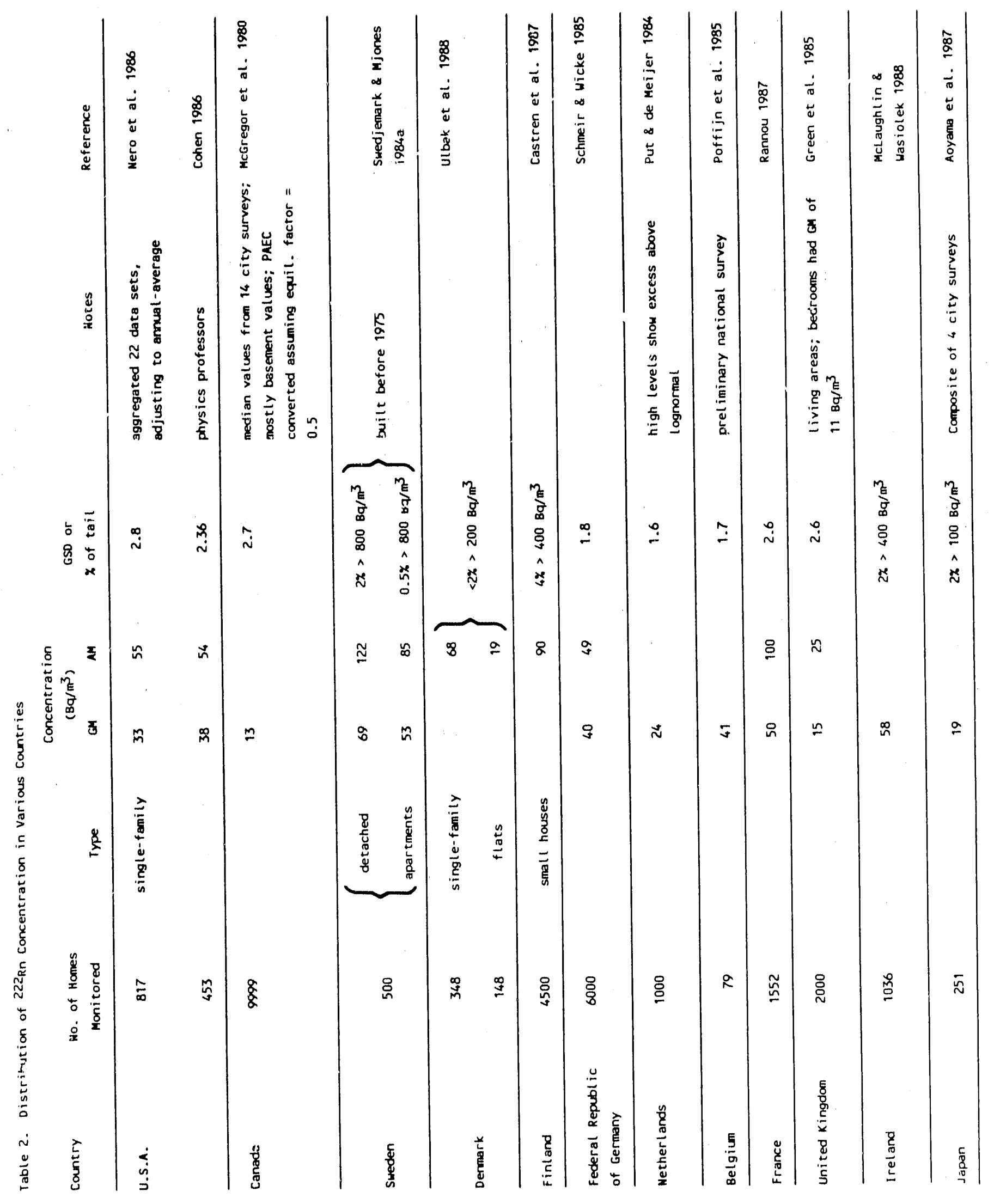


that associated studies examine relationships between average and peak concentrations under well-characterized conditions. As seen below, another incentive for emphasizing the radon concentrations per se is that this parameter appears to be a relatively direct indicator for use in strategies for identifying and controlling excessive concentrations and, as discussed elsewhere (James 1988), it may actually be a more consistent indicator of dose than the decay-product concentration - at least based on current dosimetric models.

\section{A. Studies Indicating the Concentration Distribution in U.S. Housing}

Despite a broad range of U.S. efforts to characterize indoor radon, including a substantial number of studies that have included measurements in existing U.S. homes, there has been no direct broad-scale determination of the concentrations to which the U.S. population is exposed. The numerous U.S. studies have varied significantly in incentives, scientific objectives, selection of homes, and measurement procedures. The nature of the results from these studies therefore vary significantly, as do the conclusions that may be drawn from them. An obvious solution to this difficulty would be to carry out measurements in a valid statistical sampling of U.S. homes. From even an approximate evaluation of the data already in hand, it is clear that measurements in on the order of 1000 homes would determine the mean concentration very nccurately and ascertain the fraction of homes at high concentrations (say, 5 times the mean) to a reasonable accuracy. Such a survey involving several thousand homes - is now being conducted by the EPA.

However, regardless of the potential importance of such an effort, the data already available are quite substantial, including tens of data sets from a wide variety of studies undertaken years ago and one data set obtained from a national sampling with a year-long measurement. The results from these earlier efforts constitute a reasonable basis for considering the scope of the radon problem in the United States and, hence, for formulating an effective strategy for controlling excessive exposures to radon decay products.

It had been clear for some time before the discovery of very high indoor concentrations on the "Reading Prong" in Pennsylvania that a systematic analysis of available data would have considerable value, and such an analysis was performed on data available as of 1984-1985 (Nero et al. 1986). This analysis explicitly considered important differences between various surveys and aggregated the resulting data sets, using both nonparametric and lognormal representations to yield a nominal radon distribution for single-family houses (the dominant type of residence) in the United States. The analysis handled the diverse natures of the total of 38 data sets included by (1) discriminating between those that were undertaken because high concentrations were expected, and those that were not, (2) normalizing data taken in the winter to an approximate annual-average concentration, since this is the result of principal interest for exposure (and risk) assessment, and (3) weighting the different data sets by number of houses, region, and population, in order to examine the sensitivity of the resulting distribution to aggregation method.

Figure 1 showed the probability distribution obtained from direct aggregation of 19 of the data sets for which there was no prior expectation of high radon concentrations and for which individual data were available. This particular aggregation, while not suitable for drawing conclusions about annual-average concentrations, indicates the substantial conformance of indoor radon concentrations to a lognormal representation, a result that has been observed in many individual studies.

The analytical effort examined a total of 38 data sets, corresponding to different areas (usually a state or urban area) of the United States. A significant number of these sets 
arose from monitoring efforts that were prompted by some prior knowledge of a potential for elevaten concentrations. However, the main conclusions of the analysis were based on 22 data sets apparently lacking such prior indication. In either case (i.e., the 38 sets or the 22-set grouping), the geometric mean (GM) concentrations from the individual sets ranged from 11 to $210 \mathrm{~Bq} / \mathrm{m}^{3}$ (actually, $0.3-5.7 \mathrm{pCi} / 1$, since all of these studies used the traditional units). Geometric standard deviations (GSDs) ranged from 1.3 to 4 among all 38 sets. The results of aggregating these sets in different manners are fairly insensitive to the manners of aggregation, with the main distinction being simply that aggregations utilizing the full 38 sets yielded a somewhat higher aggregate mean than those including only the 22 "unbiased" sets.

The overall result, relying on the 22-set aggregations (and including a normalization of data taken only during heating seasons to obtain an estimate of year-long averages; see Section III.A) is a distribution of annual-average ${ }^{222} \mathrm{Rn}$ concentrations that averages $55 \mathrm{~Bq} / \mathrm{m}^{3}(1.5$ $\mathrm{pCi} / \mathrm{l})$ and that has a long tail with $1-3 \%$ of homes exceeding $300 \mathrm{~Bq} / \mathrm{m}^{3}(8 \mathrm{pCi} / \mathrm{l})$. The analysis indicates that $7 \%$ (or about 4 million) of the U.S. single-family housing stock have concentrations greater than $150 \mathrm{~Bq} / \mathrm{m}^{3}(4 \mathrm{pCi} / 1)$. Alternatively, the distribution can be expressed as a lognormal function with a GM of $33 \mathrm{~Bq} / \mathrm{m}^{3}(0.9 \mathrm{pCi} / 1)$ and a GSD of 2.8 , as shown in Figure 4. (The distribution is represented by a straight line, the form which a lognormal distribution takes when one plots the logarithm of the concentration versus the percentage of homes having less than that concentration on the probability scale indicated.)

This result can only be associated with the portion of the housing stock consisting of singlefamily houses, since $99 \%$ of the data are drawn from such houses. These are, however, the dominant element of the U.S. housing stock, and the results indicate that on the order of a million homes in the United States have annual-average ${ }^{222} \mathrm{Rn}$ concentrations of $300 \mathrm{~Bq} / \mathrm{m}^{3}$ or more.

Another interesting observation from this analysis is that the geometric means of the "unbiased" 22 sets are themselves lognormally distributed, with a GSD of 2.0. This index demonstrates the substantial variability of mean indoor radon concentrations from one area to another. This variability has substantial importance in interpreting data on indoor radon concentrations - or in representation of the frequency distribution of concentrations - and in formulating control strategies. It is discussed below (Sertion C) in the context of estimating the fraction of the housing stock having high concentrations.

The distributional parameters indicated above have, of course, significant uncertainties, partly due to statistical limitations and partly because no systematic method was employed in selecting the homes to be monitored. The mere fact that the data are from groups of homes selected in 22 (or 38 ) different areas suggests that the results have some utility, though no estimate of the uncertainty due to potential bias in selecting houses can be made. The statistical uncertainty can be estimated merely by noting that 22 independent results, with a GSD of about 2, can be shown to imply an uncertainty in the aggregate mean of approximately $15 \%$. The uncertainty due to sampling bias would raise the total uncertainty above this level. It is also worth noting that the 22 sets included a total of 817 homes (of which 552 are shown in Figure 1), but this number cannot be used as the basis for estimating the statistical uncertainty, since they were grouped by region and weighted as groups in the aggregation method employed (Nero et al. 1986).

At about the same time that this analysis was being performed, an important survey was being undertaken on a nationwide basis, performing an integrated year-long measurement in the homes of 453 physics faculty at 101 colleges and universities across the United States 


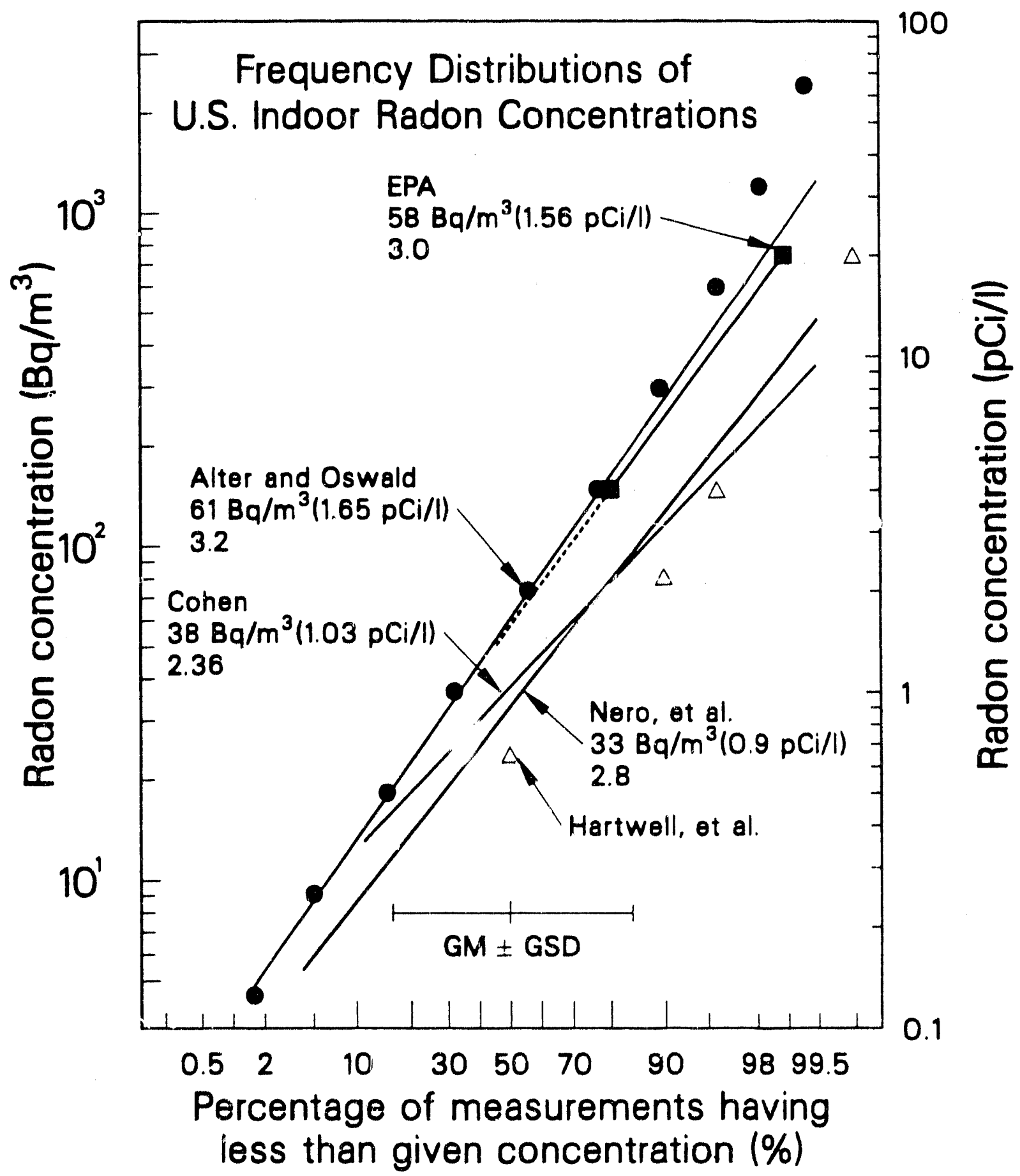

XC. $8711-11463$

Figure 4. U.S. radon distributions

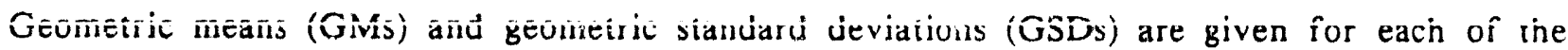
lognormal functions; see text for discussion. 
(Cohen 1986). The ${ }^{222} \mathrm{Rn}$ concentrations obtained average $54 \mathrm{~Bq} / \mathrm{m}^{3}(1.47 \mathrm{pCi} / \mathrm{l})$ and can be represented by a lognormal distribution admirably well, with a GM and GSD of 38 $\mathrm{Bq} / \mathrm{m}^{3}$ and 2.36 , respectively (cf. Figure 4 ). This implies that $0.8 \%$ of homes have concentrations greater than $300 \mathrm{~Bq} / \mathrm{m}^{3}$, at the low end of the range estimated from the analysis of Nero et al. (1986). On the other hand, the fraction above $150 \mathrm{~Bq} / \mathrm{m}^{3}, 6 \%$, is very close to the earlier result. Although a study of homes of physics faculty cannot be construed to be a representative sampling of U.S. homes (a problem suffered also by the aggregate analysis just discussed), this study has the advantage that the homes are widely distributed across the United States and that the measurement protocol determined directly a year-long average concentration. The averages from the 101 institutions are themselves lognormally-distributed, with a GM of $46 \mathrm{~Bq} / \mathrm{m}^{3}$ and a GSD of 1.75 . Interestingiy, the highest values of geographical averages from both this study and Nero et al. (1986) occurred in the state of North Dakota, in particular in cities in the Red River Valley.

A significant number of the earlier studies analyzed in Nero et al. (1986) measured the concentration of decay products instead of radon (though the primary grouping of 22 sets relied almost entirely on etched-track detectors). One of these in particular measured both concentrations averaged over week-iong periods, thereby determining directly an effective equilibrium ratio, which was found to range from 0.31 to 0.82 among the houses examined (George and Breslin 1980). As will be evident from the measurements from other countries, discussed below, there is significant evidence that aquilibrium ratios tend to average about 0.4 among samples of housing, with significant variability from one time to another, from one house to another, and perhaps some differences from one region or country to another.

We note another important study that was undertaken prior to 1985 , but the results of which have only begun to be available recently, namely a statistically-designed survey of radon concentrations in more than 2000 homes in New York State (Hartwell et al. 1987). The annual-average living space concentrations resulting from that work are illustrated in Figure 4, along with the distributional forms from the work discussed above. The New York results are, for the most part, slightly lower than the tentative nationwide distributions from Nero et al. (1986) and Cohen (1986), as indicated by either the distribution in the figure or the average for the New York results, about $42 \mathrm{~Bq} / \mathrm{m}^{3}(1.1 \mathrm{pCi} / \mathrm{l})$. On the other hand, aggregation of the several New York data sets that appear among the 22-set grouping of Nero et al. (1986) yields an average of about $40 \mathrm{~Bq} / \mathrm{m}^{3}(1 \mathrm{pCi} / 1)$, similar to the average from the New York State survey (which, however, yielded annual-average concentrations). And the direct average of concentrations in the 16 New York homes of physics professors monitored by Cohen $(1986)$ is $30 \mathrm{~Bq} / \mathrm{m}^{3}(0.8 \mathrm{pCi} / \mathrm{l})$. These comparisons indicate that, at least for the one state-wide survey that is designed to yield annual-average concentrations in the living space, the rejults are in the same range as those that served as a partial basis for the U.S. distribution discussed earlier.

At present, it is the analysis of Nero et a!. (1986) and the data of Cohen (1986) that provide our present appreciation of the U.S. concentration distribution in residences, and principally in single-family houses. As noted above, these efforts both yield average concentrations of $55 \mathrm{~Bq} / \mathrm{m}^{3}$ and substantial conformance to a lognormal distribution. As seen in Figure 4 , the percentage of homes having more than $150 \mathrm{~Bq} / \mathrm{m}^{3}(4 \mathrm{pCi} / 1)$, the EPA's primary guideline for remedial action (USEPA 1986), is about $6 \%$ for either distribution. The two distributions diverge significantly as the concentration increases to higher levels, so that the percentages having concentrations greater than, say, $300 \mathrm{~Bq} / \mathrm{m}^{3}(8 \mathrm{pCi} / 1)$ are approximately $1.8 \%$ and $0.8 \%$. respectively. from Nero et al. (1986) and Cohen (1986). Extrapolating to $740 \mathrm{~Bq} / \mathrm{m}^{3}(20 \mathrm{pCi} / \mathrm{l})$ yields, respectively, $0.13 \%$ and $0.02 \%$, a substantial difference, but in 
either case a small percentage. These results contrast strongly with those from the large data bases of indoor radon concentrations that have been accumulated subsequent (and due) to the discovery of very large concentrations in homes on the Reading Prong geologic formation in eastern Pennsylvania. As we will see below, the large fractions of homes of ten cited to exceed either the EPA guideline or $740 \mathrm{~Bq} / \mathrm{m}^{3}$ (at which level residents would receive annual exposures comparable to the occupational limit) are almost always wrong, resulting from surveys or data compilations that do not yield annual-average concentrations in the living spaces of representative sets of homes.

\section{B. More recent data on U.S. residences}

By the early 1980s it had been clear that indoor radon levels were high enough to warrant real concern. Hence the monitoring efforts cited in the previous section. Not only did it already appear that the bulk of U.S. houses had concentrations in the range of 20 to 100 $\mathrm{Bq} / \mathrm{m}^{3}$ (i.e., about 0.5 to $3 \mathrm{pCi} / \mathrm{l}$ ), thus indicating all average toward the central part of this range, but a significant number of homes had already been found to have much higher concentrations, though not in a representative survey. For example, of perhaps three or four thousand measurements in U.S. homes that the research community had performed by 1984 , between 10 and 20 had yielded results in the range of 2000 to $6000 \mathrm{~Bq} / \mathrm{m}^{3}$ (about $5 \mathrm{c}$ to $150 \mathrm{pCi} / \mathrm{l})$, an extraordinarily high proportion having about 100 times the average. This caused speculation about when the first home having 1000 times the average level would be found. It happened within a year, is: eastern Pennsylvania!

Since very high concentrations had already been found in eastern Pennsylvania (Sachs et al. 1982), this was something of a rediscovery. But it galvanized the political and regulatory community into finally paying substantial attention to the problem of indoor radon. It has also resulted in very large numbers of measurements being performed in U.S. homes, more than a million by the date of this publication.

Unfortunately, this very large amount of monitoring has not greatly improved our appreciation of the frequency distribution. In fact, due to the way that information has been presented to the public, and even to the scientific community, a substantial distortion has occurred. As a result, many have a greatly inflated notion of what average concentrations are and of how frequentily homes have levels exceeding the EPA guideline or higher levels, such as the $740 \mathrm{~Bq} / \mathrm{m}^{3}$ that corresponds roughly to the limit of $4 \mathrm{WLM} / \mathrm{y}$ that applies to exposure rates in occupational settings. It is therefore important to examine certain of the large data sets that have become available, in order to understand how they have been misinterpreted or misused. Furthermore, it is useful to see what new information can be obtained from these data, in spite of their deficiencies for general assessment purposes.

As noted above, the monitoring technique that is most suitable for determining the average radon concentration in a structure over a substantial period is to deploy an etched-track detector, which can remain in place even for a full year, though they are most frequently used for a period of a month to several months. By 1986, the use of such detectors had resulted in a substantial data base, and the results from approximately 60,000 measurements in U.S. homes were examined to indicate a nationwide distribution (Alter and Oswald 1987). The average of these results was found to be $266 \mathrm{~Bq} / \mathrm{m}^{3}(7.2 \mathrm{pCi} / \mathrm{l})$, but 50,000 of these results arose from only 6 states, including some where monitoring programs have been undertaken because of the existence of high concentrations. Removing these states yields an average, for the 10,251 remaining results, of $158 \mathrm{~Bq} / \mathrm{m}^{3}(4.26 \mathrm{pCi} / \mathrm{l})$. Two alternative methods of obtaining a more representative selection or weighting yield averages of 152 and 
$153 \mathrm{~Bq} / \mathrm{m}^{3}$. The authors find this convergence striking, assert that the results provide a rough "exposure" estimate, and conclude that the national "exposure" exceeds that cited previously, e.g., the $55 \mathrm{~Bq} / \mathrm{m}^{3}$ average indoor concentration from Nero et al. (1986). It is striking, in fact, that the average from Alter and Oswald (1987) approximates the remedial action limit of $150 \mathrm{~Bq} / \mathrm{m}^{3}$ recommended by the EPA.

In point of fact, even the three restricted data sets are likely to have irremovable overrepresentation of homes with high concentrations, simply because of repetitive sampling in homes or areas where high levels are found and the fact that such oversampling has not been removed from the data set. Furthermore, the parameter of interest for indoor exposures is the annual-ave iage concentration in the living space, and the data set of Alter and Oswald (1987) has not been corrected to yield annual-average values and has not been edited on the basis of location. As an illustration of the outcome of using such data, we indicate here (and in Table 3 ) the probable scale of error in using the results as in Alter and Oswald (1987).

A major difficulty is that a large portion of recent measurements have been performed in basements that are not primary living space. Many U.S. homes have basements, especially in the areas where a great deal of monitoring has been performed (such as eastern Pennsylvania and New Jersey), and the protocol recommended by the EPA for "screening" specifies monitoring in basements where they exist (USEPA 1986). However, concentrations in basements average approximately twice those on the first floor during the winter, with an even larger ratio during the summer (Cohen 1987 and Hartwell et al. 1987). In fact, even Alter and Oswald (1987) describe results from a subset of their measurements in which location was recorded, finding that $44 \%$ were taken in basements and have an average concentration 2.0 times the average from the non-basement readings.

If we attempt to correct an apparent average of $155 \mathrm{BG} / \mathrm{m}^{3}(4.2 \mathrm{pCi} / 1)$ to a living-space average assuming that $44 \%$ have results that are a factor of 2 higher than the living-space value, we find a corrected average of $108 \mathrm{~Bq} / \mathrm{m}^{3}(2.9 \mathrm{pCi} / \mathrm{l})$, a large change. It is even more difficult, and probably impossible, to estimate the effect of oversampling in areas or houses where high concentrations are known or suspected. We note that a mere $25 \%$ reduction of the average yields $81 \mathrm{~Bq} / \mathrm{m}^{3}(2.2 \mathrm{pCi} / 1)$; as will be discussed elsewhere, there are indications from the etched-track data themselves and from comparison with the EPA data discussed below that the effect of oversampling is at least this large. Finally, due to the nature of monitoring programs and recommendations, a preponderance of measurements are performed during winter only. As indicated in Section III.A, annual-average concentrations have been found to average approximately $72 \%$ of winter values based on four surveys (Nero et al. 1986). Given probable mixes of seasons in which the etched-track measurements were taken, the annual-average concentrations may therefore be 80 or $90 \% \mathrm{cf}$ the value above, i.e., approximately $69 \mathrm{~Bq} / \mathrm{m}^{3}(1.9 \mathrm{pCi} / \mathrm{l})$.

The above estimate is not intendied to represent an actual corrected value, because there is not enough information to adjust the recults quantitatively. However, these considerations are enough to indicate that, based on the results from Alter and Oswald (1987), an average of less than $75 \mathrm{~Bq} / \mathrm{m}^{3}(2 \mathrm{pCi} / 1)$ - and therefore consistent with the earlier results - is probable, and that the values cited in the paper itself give a wholly misleading indication of public exposures.

Another major result of Alter and Oswald (1987) is the implication that large numbers of homes have high concentrations; e.g., $23 \%$ of the 10,251 data in the "U.S. minus 6 states" data set exceed $150 \mathrm{~Bq} / \mathrm{m}^{3}$, the EPA remedial action recommendation. These results are also plotted on Figure 4, where we see that a lognormal function with a $\mathrm{GM}$ of $61 \mathrm{~Bq} / \mathrm{m}^{3}$ 
Table 3. Comparative characteristics of U.S. radon distributions

\begin{tabular}{lccccc} 
& Sample Size & $\begin{array}{c}\mathbf{G M} \\
\left(\mathbf{B q} / \mathbf{m}^{3}\right)\end{array}$ & GSD & $\begin{array}{c}\text { AM } \\
\left(\mathbf{B q} / \mathbf{m}^{3}\right)\end{array}$ & $\begin{array}{c}\text { "Corrected" } \\
\mathbf{A M}\left(\mathbf{B q} / \mathbf{m}^{3}\right)\end{array}$ \\
\hline $\begin{array}{l}\text { Nero et al. } \\
1986\end{array}$ & 817 & 33 & 2.8 & 55 & \\
Cohen 1986 & 453 & 38 & 2.36 & 54 & $70 \pm 35 ?^{\mathrm{a}}$ \\
$\begin{array}{l}\text { Alter and } \\
\text { Oswald 1987 }\end{array}$ & 10,251 & 64 & 3.48 & 158 & \\
USEPA 1987 & 10,000 & $(58)^{\mathrm{b}}$ & $(3.0)^{\mathrm{b}}$ & 110 & $55^{\mathrm{c}}$
\end{tabular}

a Raw average corrected by factors of 0.75 for overrepresentation of high-concentration homes and 0.85 for approximate correction from winter to annual-average concentrations in majority of homes. Result is then divided by 1.44 to correct for $44 \%$ of data taken in basements (based on Alter and Oswald 1987), where concentrations are approximately two times higher than in living space.

b Lognormal parameters for lognormal function drawn between EPA data points, as on Figure 4.

c Raw average corrected by factor of 0.72 (from Nero et al. 1986), since all results were in winter, and divided by 1.44 , assuming same fraction of basements as in Alter and Oswald (1987). 
( $1.65 \mathrm{pCi} / \mathrm{l}$ ) and a GSD of 3.2 fits the points up to $150 \mathrm{~Bq} / \mathrm{m}^{3}$ very well, but that there is a very large excess of results with higher concentrations, especially at very high values. The considerations discussed above would appear to account for the fact that this curve is substantially above those of earlier studies, as well as for a substantially inflated percentage of houses exceeding $150 \mathrm{~Bq} / \mathrm{m}^{3}$.

Results of considerable interest have also arisen from recent efforts of the EPA in conjunction with a number of states. For example, during the winter of 1986-87, charcoal detectors were deployed in statistically chosen samples of homes in nine states, plus another state in which volunteers were relied on. The results of surveys in these ten states were reported in a press conference in August, 1987 and in associated materials (USEPA 1987). From the press materials, one finds the following information: the number of homes monitored in the main sample varied from 190 to 1787 for the ten states, totaling approximately 10,000. (Additional samples were taken on a volunteer basis, in at least one case to investigate areas thougnt to have a potential for higher concentrations than average.) The average concentration for the main sample was $110 \mathrm{~Bq} / \mathrm{m}^{3}(3.0 \mathrm{pCi} / 1) ; 20 \%$ of measurements exceeded $148 \mathrm{~Bq} / \mathrm{m}^{3}(4.0 \mathrm{pCi} / 1)$ and approximately $1.1 \%$ exceeded $740 \mathrm{~Bq} / \mathrm{m}^{3}$ $(20 \mathrm{pCi} / 1)$. Measurements were taken following the EPA screening protocol and using 2day sampling during the heating season. A large proportion of the sampling was performed in basements. Thus these results do not represent annual-average concentrations to which people are exposed. Nonetheless, the press reports, in accordance with the EPA press release, compared these results directly with the EPA action guideline and indicated that $21 \%$ of (the total of) 11,600 homes exceeded the guideline. This conclusion is obviously wrong, based on an inappropriate use of the data, but unfortunately has had a large role in forming public and policy perceptions of the problem of indoor radon.

The data, however, could be useful in other respects. On Figure 4 are plotted (solid squares) the probabilities corresponding to 148 and $740 \mathrm{~Bq} / \mathrm{m}^{3}$. The lognormal function passing through these points has a GM of $58 \mathrm{~Bq} / \mathrm{m}^{3}(1.56 \mathrm{pCi} / 1)$ and a GSD of 3.0 , for which the corresponding arithmetic mean is $106 \mathrm{~Bq} / \mathrm{m}^{3}(2.9 \mathrm{pCi} / 1)$. This is close enough to the actual average reported to suggest, based on the limited information available (EPA 1987), that this lognormal function may be a useful representation of the data. If so, it is interesting to note that lognormality is maintained at least up to the region of $740 \mathrm{~Bq} / \mathrm{m}^{3}$. This contrasts strongly with the data of Alter and Oswald, and others, who report raw or uncontrolled data, but is consistent in this respect with the data of Nero et al. (1986) and Cohen (1986). It is important, in this context, to realize that - for the most part - the EPA data arise from a statistical sampling, thus avoiding the over-representation of high areas that is characteristic of most of the recent large data sets.

Tiat the EPA distribution as a whole is higher that the earlier results is not surprising, considering the monitoring protocol. As an illustration (Table 3), if the same correction from basement concentrations as above is made to the arihmetic mean of $110 \mathrm{~Bq} / \mathrm{m}^{3}$, and the resulting $76 \mathrm{~Bq} / \mathrm{m}^{3}$ is corrected to an annual-average value using the factor of 0.72 from Nero et al. (1986), an average of $55 \mathrm{~Bq} / \mathrm{m}^{3}$ is found. The precise agreement with earlier results is fortuitous, but indicates that - if proper adjustments could be made agreement would be quite satisfactory. Furthermore, these adjustments would dramatically reduce the fractions above 148 and $740 \mathrm{~Bq} / \mathrm{m}^{3}$, probably to the vicinity of previous results, which were that perhaps $6 \%$ of homes exceed $148 \mathrm{~Bq} / \mathrm{m}^{3}$ and perhaps $0.1 \%$ exceed 740 $\mathrm{Bq} / \mathrm{m}^{3}$.

The EPA data also give the first useful indication of the shape of the distribution at even higher concentrations, at least for these ten states. Extrapolating the lognormal representation (of Figure 4) to high levels indicates that approximately $0.01 \%$, i.e., a single 
home out of the 10,000 , ought to have exceeded $3700 \mathrm{~Bq} / \mathrm{m}^{3}(100 \mathrm{pCi} / \mathrm{l})$. The number actually found in the EPA/state surveys was three, which is reasonably close. If adjustments were made to annual-average concentrations in living spaces, one would expect from this data set that a lower number, i.e., fewer than 3 in 10,000 homes, would exceed $3700 \mathrm{~Bq} / \mathrm{m}^{3}$. This contrasts strongly with the results of Alter and Oswald (1987) and others who find much higher fractions exceeding this level, basically tecause their data are not representative. The fact that most data are also taken with the EPA screening protocol suggests that the fraction of houses having annual-average living-space concentrations greater than this level will be even lower than the upper limit indicated here.

This is not to suggest that the extreme high-concentration tail of the distribution of U.S. concentrations does not exceed a lognormal representation. The ten states in the initial EPA survey may turn out to have less variance among them than the U.S. as a whole. The question of the high concentration tail is examined in more detail below, but it is worth mentioning at this point that some of the 1987 EPA data have been reported in more detail, including the actual frequency distributions and comparisons of certain subsets (RoncaBattista et al. 1988) for five of the ten states. One of the interesting observations from these results is that, although the data differ substantially from one state to another, examination of results from basements versus first floors (in houses without basements) indicates that the differences between these states may arise primarily from differences in the fractions of houses having basements. Furthermore, if parallel sets of data from Alabama are any indication, results from volunteers are on the average about $50 \%$ greater than results from randomly selected houses, for reasons that are not understood.

In 1988, the EPA announced the results from surveys in seven more states, unfortunately in a manner that was open to wide and substantial misinterpretation (USEPA 1988). Thus it was said that in these states nearly one in three homes had screening levels exceeding the EPA guideline, and that "over 200,000 homes in these states will have levels giteater than 20 $\mathrm{pCi} / 1$, a level that exceeds current health-protection standards for uranium miners." However, the press release and resulting stories did not indicate 1) that most of the homes with screening levels above $150 \mathrm{Ba} / \mathrm{m}^{3}(4 \mathrm{pCi} / 1)$ will be found not to exceed the EPA guideline, which pertains to annual-average concentrations in living areas, and 2) that a very small fraction of the 200,000 homes cited will actually be found to cause annual exposures exceeding those for uranium miners. Because of such omissions, the public release of information on the surveys the EPA has conducted in cooperation with the states has given a highly misleading impression of the nature and scope of the indoor radon problem. Unfortunately this impression is reinforced by the manner in which some scientists also represent the current understanding, examples being the paper on the etchedtrack data base discussed above (Alter and Oswald 1987) and subsequent representations of these data (Alter and Oswald 1989).

We mention one other major data base, that developed by Cohen and coworkers at Pittsburgh. There the "Radon Project" has performed very large numbers of measurements using charcoal sampling techniques, and the results reported using various geographic scales, e.g., as presented in Cohen (1987). In a more recent table of "Radon Levels by States and Counties," the results of some 38,872 measurements in living areas are reported, averaging $122 \mathrm{~Bq} / 3.3 \mathrm{pCi} / 1)$, with a $\mathrm{GM}$ of $61 \mathrm{~Bq} / \mathrm{m}^{3}(1.7 \mathrm{pCi} / 1)$ and with no report of the GSD (Cohen 1309$)$. Approximately $17 \%$ of the results exceed $148 \mathrm{~Bq} / \mathrm{m}^{3}(4 \mathrm{pCi} / 1)$, while $2 \%$ exceed $740 \mathrm{~Bq} / \mathrm{m}^{3}(20 \mathrm{pCi} / \mathrm{l})$. While the derailed data, given by county (or even by ZIP code in an alternative form of the table), may be useful in determining relative concentrations, the resilts as a whole appear to be substantially higher in concentration than those reviewed in the previous section, although not as great as the etched-track data just discussed or the results from the EPA/state surveys. In particular, the average is 
approximately twice that from the earlier studies discussed above, and the fractions greater than 150 or $740 \mathrm{~Bq} / \mathrm{m}^{3}$ are also considerably greater than in those studies.

An illuminating comparison may be made on the basis of the data for the state of New York, for which Cohen (1989) reports an average of $70 \mathrm{~Bq} / \mathrm{m}^{3}(1.9 \mathrm{pCi} / \mathrm{l})$, with $9 \%$ greater than $4 \mathrm{pCi} / 1\left(148 \mathrm{~Bq} / \mathrm{m}^{3}\right)$. This contrasts with the average of $41 \mathrm{~Bq} / \mathrm{m}^{3}(1.1 \mathrm{pCi} / \mathrm{l})$, with $4.2 \%$ exceeding $4 \mathrm{pCi} / 1\left(148 \mathrm{~Bq} / \mathrm{m}^{3}\right)$, available from the New York State survey (Hartwell et al. 1987). These differences occur, presumably, because the "Radon Project" data are not representative of the housing stock.

Finally, results have recently become available from two major studies undertaken in New Jersey as a result of the Reading Prong discoveries. First, an epidemiological study of lung cancer among women in New. Jersey included radon concentration measurements in homes of approximately 400 cases and 400 controls (NJSDH 1989). For most homes, measurements included both a year-long etched-track measurement and a short-term charcoal sampling (in basements where present). Although the average concentration is not given for either set of results, only 8 of the year-long concentration results exceeded $148 \mathrm{~Bq} / \mathrm{m}^{3}(4 \mathrm{pCi} / 1)$, i.e., about $1 \%$. This contrasts strongly with the charcoal results, where $13 \%$ of the measurements exceeded $148 \mathrm{~Bq} / \mathrm{m}^{3}(4 \mathrm{pCi} / \mathrm{l})$ and illustrates the degree to which the prevalence of high concentrations can be overestimated by using the EPA screening protocol.

The second recent New Jersey study to be mentioned is a broad study of concentrations in houses, intended to characterize the occurrence of radon in New Jersey homes and to examine correlations with causative factors (CDM 1989). The survey component of the study yielded results from approximately 6000 homes, with the intensity deliberately chosen to be greater in areas on New Jersey where geology indicated concentrations ought to be higher. The average concentration of the raw data, obtained using charcoal canisters and a screening protocol, was $190 \mathrm{~Bq} / \mathrm{m}^{3}(5.2 \mathrm{pCi} / \mathrm{l})$, with $32.5 \%$ of the results exceeding 148 $\mathrm{Bq} / \mathrm{m}^{3}(4 \mathrm{pCi} / 1)$. If the results from different areas are weighted according to population, the statewide average was $90 \mathrm{~Bq} / \mathrm{m}^{3}(2.5 \mathrm{pCi} / 1)$ and the percentage exceeding $148 \mathrm{~Bq} / \mathrm{m}^{3}(4$ $\mathrm{pCi} / 1$ ) was $14 \%$ (as estimated in NJSDH 1989). Thus the fraction above the EPA action level, as measured in a screening protocol, is similar in these two studies, and - in turn - is much greater than the $1 \%$ of homes found in NJSDH (1989) to exceed the action level when annual-average concentrations are measured in the living space. (On the other hand, the two studies yield substantially different, albeit small, proportions of homes exceeding 740 $\mathrm{Bq} / \mathrm{m}^{3}(20 \mathrm{pCi} / 1)$ using a screening protocol - i.e., $0.1 \%$ from the epidemiological study and $1.6 \%$ from the statewide radon characterization. This discrepancy may arise in part from changes in population distribution since the time when the case-control study subjects occupied the houses that were monitored.) Finally, the fact that the characterization study yields an average concentration of about $90 \mathrm{~Bq} / \mathrm{m}^{3}(2.5 \mathrm{pCi} / 1)$, based on a screening protocol that typically yields values averaging 2 or 3 times the annual-average living-space concentration, suggests that the average concentration to which New Jersey residents are exposed in their homes is in the vicinitv of $40 \mathrm{~Bq} / \mathrm{m}^{3}$ (about $1 \mathrm{pCi} / 1$ ). This is similar to the results from the New York survey discussed in the previous section, and less than the tentative national average of $55 \mathrm{~Bq} / \mathrm{m}^{3}$. This occurs in spite of the fact that parts of New Jersey are known to have some of the highest indoor concentrations in the United States, and in fact in the world, as discussed in the next section.

A comparison of the charcoal ar.d etched-track results from the New Jersey epidemiological study gives a disconcerting result for the ratio of these two types of data. That is, for the lower concentrations, the "screening" results (from a charcoal canister in the basement) exceed the year-long living-space measurements (taken with an etched-track detector) by approximately a factor of 2 . However, for the "screening" results exceeding 150 and 300 
$\mathrm{Bq} / \mathrm{m}^{3}$ (i.e., 4 and $8 \mathrm{pCi} / \mathrm{l}$ ), the difference grows to a factor of 5 , indicating that, at least for the homes examined in this study, high results from the screening protocol tend to be grossly exaggerated.

\section{The occurrence of high concentrations or exposures}

The previous sections provide a basic picture of the distribution of indoor radon concentrations, i.e., that the average concentration in single-family residences is approximately $55 \mathrm{~Bq} / \mathrm{m}^{3}$, that about $6 \%$ of houses have concentrations exceeding 150 $\mathrm{Bq} / \mathrm{m}^{3}$, and roughly $1 \%$ have levels above twice this value. Limited data suggest that as the level of concern increases, the fraction of houses above it falls very rapidly, so that the fraction of houses with levels exceeding $740 \mathrm{~Bq} / \mathrm{m}^{3}$ appears to be only about $0.1 \%$. Monitoring data also show a marked dependence of the distribution on geographic area, so that the broad distribution just indicated does not indicate the much greater occurrence of high values that may occur in specific areas. In this section, we examine the extent to which we can understand, on present evidence, the high-concentration tail of the national distribution and the variability of local distributions from one place to another. We also indicate the potentially great difference between the distribution of concentrations in homes and the frequency distribution of human exposures. All of these questions have substantial implications for formulating risk-limiting objectives and for designing control strategies, the subject of Section VI.

The various efforts discussed above, although adequate for indicating the frequency distribution of low to moderately high concentrations, do not permit an unequivocal estimate of the frequency of occurrence of homes having very high concentrations. Even for the percentage of houses with concentrations exceeding only $740 \mathrm{~Bq} / \mathrm{m}^{3}(20 \mathrm{pCi} / 1)$, estimates are difficult because, first, the data of Nero et al. (1986) and Cohen (1986) do not include enough houses to provide sound estimates of occurrence at such levels and, secondly, the other data bases that are available, as discussed in the previous section, are not based on annual-average concentrations in living space. Thus, as noted above, the Nero et al. (1986) and Cohen (1986) results suggest the fraction above this level to be 0.13 and $0.02 \%$, respectively, while the EPA 1987 result of $1.1 \%$ must be disregarded, considering the monitoring protocol employed. The other survey cited, that in New York (Hartwell et al. 1987), is relevant, since it had a distributional form that was only slightly different from those of Nero et al. (1986) and Cohen (1986) - as seen in Figure 4. The New York survey found only $0.2 \%$ of homes to have concentrations exceeding $740 \mathrm{~Bq} / \mathrm{m}^{3}$, in contrast with the 1987 EPA results or the even larger fractions of 3 or $4 \%$ from the Alter and Oswald (1987) data base and $2 \%$ from Cohen (1989).

The uncertainty in estimates becomes even larger for fractions of houses at higher levels, e.g., above $3700 \mathrm{~Bq} / \mathrm{m}^{3}(100 \mathrm{pCi} / \mathrm{l})$. Although a number of houses with heating season values of this magnitude had been found prior to $1984-85$, it was the intense monitoring in eastern Pennsylvania, following discovery of the house having of the order of 100,000 $\mathrm{Bq} / \mathrm{m}^{3}$, that led to identification of numerous houses with levels greater than $3700 \mathrm{~Bq} / \mathrm{m}^{3}$. These efforts have, in turn, led to the impression tlat a substantial number of U.S. houses have such high levels, in contrast to the approximately 2 in a million that would be estimated by extrapolating the distribution of Nero et al. (1986) to such high concentrations. Such extrapolation would only be valid if the lognormal distribution were an adequate representation of the actual distribution even for such high levels, a possibility for which there is no direct evidence, considering the relatively small number of homes treated in Nero et al. (1986) and Cohen (1986).

The only evidence that gives a direct quantitative indication of the probability of such high 
levels is the data from the EPA surveys where, as noted above, 3 of 10,000 data reported in 1987 (USEPA 1987) exceeded $3700 \mathrm{~Bq} / \mathrm{m}^{3}$, whereas a direct extrapolation of the data at lower levels suggusted about $0.01 \%$ should exceed this level. Of the 11,000 measurements reported in 1988 (USEPA 1988), five exceeded this level, i.e., $0.05 \%$. In contrast, approximately $0.5 \%$ of the full data base of Alter and Oswald (1987) exceed $3700 \mathrm{~Bq} / \mathrm{m}^{3}$, a full order of magnitude greater than the EPA results, presumably reflecting the substantial oversampling of high concentration houses in that data base. And, based on the charcoal data of Cohen and coworkers, $0.1 \%$ of house have been estimated to exceed this level (Cohen 1987b). However, the EPA results provide, in effect, an upper bound on the fraction having such levels, because these data are from monitoring using the EPA's screening protocol, so that they are short-term measurements, about half taken in basements. It would therefore not be surprising if, typically, the EPA results must be divided by approximately 2 to yield the annual-average value in the living space, in which case the number in their 1987 sample remaining above $3700 \mathrm{~Bq} / \mathrm{m}^{3}$ would be zero, since none of the 8 houses cited above had concentrations exceeding twice this value. Given the statistics, and a bit of speculation, it would be fair to say that the EPA results suggest that on the order of 1 house out of 20,000 may have annual-average living-space concentrations exceeding $3700 \mathrm{~Bq} / \mathrm{m}^{3}$.

This still permits the possibility that the national distribution diverges from a lognormal distribution at very high concentrations. In fact, even the 2000-house survey in New York (Hartwell et al 1987) indicates such a divergence, in that the fraction of houses exceeding $740 \mathrm{~Bq} / \mathrm{m}^{3}(20 \mathrm{pCi} / \mathrm{l})$ is greater than would be expected from an extrapolation from the lower points shown in Figure 4. To put it another way, the cumulative probability plot curves upward as the concentration increases, indicating an excess of houses with high concentrations as compared with a lognormal distribution, for which the data would fall along a straight line. A similar form has been observed in many circumstances, raising the possibility that a simple lognormal form - with parameters such as those found by Nero et al. (1986) or Cohen (1986) - that adequately represents the data for the vast bulk of houses may not suffice for the very small portion of residences that have very high concentrations and may, in fact, substantially underestimate the number of such residences.

This possibility must be considered, especially in view of the seemingly large number of houses with high concentrations that have been found in the area of the Reading Prong, primarily in eastern Pennsylvania and New Jersey. Furthermore, even the Pennsylvania data from the Reading Prong area alone suggest a distributional form that has a rising slope for higher concentrations on a cumulative probability plot. An example of such data is given in Figure 5, which shows results from a Pennsylvania mailing of etched-track devices to homes in the Reading Prong area; detectors were placed for three months, primarily in the winter (of 1985-86), in basements where there was one (Granlund 1988). Although this figure does not provide detailed information on the distributional form, particularly at lower concentrations, it is clear that the slope of the curve increases in the region of the highest concentrations. Thus, although the line, with a GSD of 2.9 , adequately represents the central probability region, its use would substantially underestimate the fraction of homes at very high levels (and overestimate the fraction at very low levels). Note that the median value from this survey is approximately $200 \mathrm{~Bq} / \mathrm{m}^{3}(5.4 \mathrm{pCi} / 1)$, considerably greater than the geometric mean of $33 \mathrm{~Bq} / \mathrm{m}^{3}(0.9 \mathrm{pCi} / 1)$ from Nero et al. (1986), reflecting both the higher-than-average concentrations in the Reading Prong and the fact that most of these detectors were placed in basements. (Note also that the GSD is similar to that from Nero et al. 1986, in contrast to other, more well-known Pennsylvania results with substantially higher GSDs, but that were not acquired in as careful a manner.) 


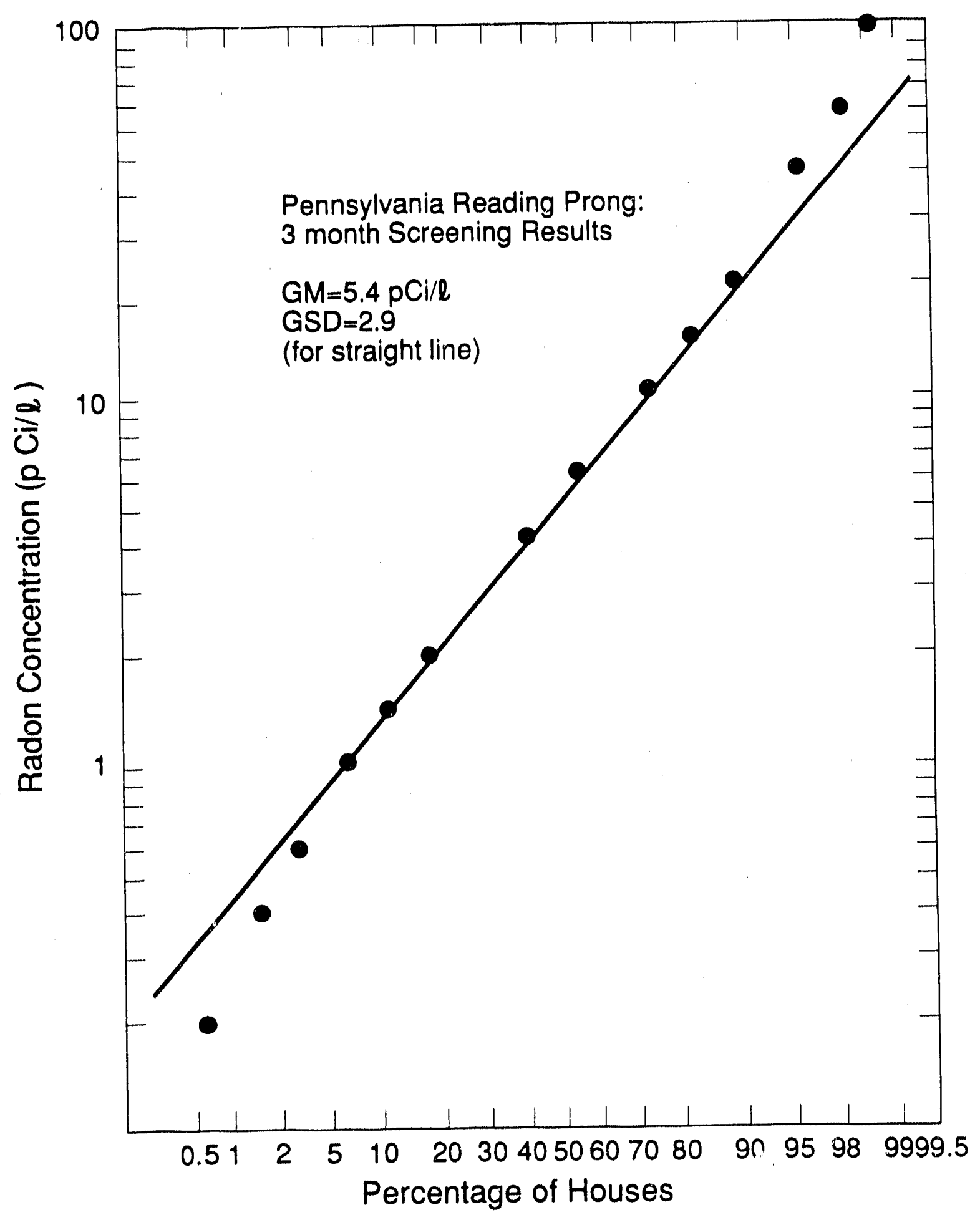

XBL 901-269

Figure 5. Distribution of ("screening") radon concentrations from Pennsylvania.

Etched track detectors were placed for approximately three months, pri: iarily in winter, and in basements where present (Granlund 1988). Approximately 17,000 home were monitored. 
Such observed excesses of high concentrations have given rise to speculation of varying kinds, one being that a supralognormal form is characteristic of areas having high concentrations. However, a counterexample is provided by the case of Clinton, New Jersey, where very high concentrations were found during 1986 (Osborne 1987). The data from Clinton are shown on Figure 6, where it is seen that they are fit well by a straight line with the lognormal parameters indicated. (Note, in this case, the very large GM and GSD.)

An alternative hypothesis is that such local or regional surveys effectively add (or "mix") two or more subpopulations that together yield a supralognormal form, the questions then being why and of what form. For example, it has been proposed (Goble and Socolow 1988) that the tail of regional or national distributions may decrease in proportion to some power of the concentration, which - if true - would imply a substantial greater fraction of the total population exposure occurring among occupants of high-concentration houses than is implied by the essentially exponential form of the tail of a lognormal distribution. As a result, the form of the tail can have significant implications for understanding risks, for designing epidemiological studies, or for formulating strategies to limit risks.

On the other hand, some type of supralognormal form is consistent with the hypothesis that indoor concentrations are well represented, in general, by lognormal functions, and that the supralognormal form results from what is in effect the addition (or "mixture") of two - or in any case a small number of - subpopulations that individually are adequately represented by single lognormal functions. This is consistent with the general hypothesis - based on the "central limit theorem" from statistics (Aitchison and Brown 1957) - that a lognormal form results when, for example, the parameter of interest is effectively the product of a large number of underlying parameters, which themselves may be distributed normally (i.e., according to a Gaussian distribution). Considering that we know, based on our physical understanding (as discussed in Section III) that the indoor concentration is in fact determined by a wide range of factors, some multiplicative and some additive, it is not surprising to find that distributional forms of ten fit lcgnorrnal functions well. In fact, it is also known that some of the causative factors are lognuimally distributed, as illustrated by the lognormal distribution of uranium and thorium concentrations in most rock types (Wollenberg and Smith 1987).

This picture also accounts for the fact that the lognormal function may serve well at any geographic scale, whether town, city, state, or nation. At any of these scales, the observable - the indoor radon concentration - is determined by a large number of underlying factors (especially in view of the fact that each primary factor, such as the soil radium concentration, is itself determined by an underlying complex of factors), but the range or particular distribution of the underlying factors differ from one geographic scale or location to another. In this picture, then, the distribution for a large region can be considered to be the sum (or mixture) of the distributions representing the smaller areas that make up that region. And, in the limit of large numbers of subareas, the regional (or national) distribution can be expected to be represented, in first order, by a single lognormal distribution - just as is true of distributions for smaller areas. On the other hand, as one acquires more and more data, so that the regional/national distribution is more precisely determined, one may find evidence of divergence from a single lognormal, particularly in the extreme tail, simply because that tail can be influenced greatly by the occurrence of one or a few areas having extraordinary concentrations.

Thus, one might expect that, when a distributional form is found, with increasing amounts of data, to diverge in the tail from a lognormal form, it can be reasonably represented in second order by a sum of two lognormal functions. One test of this hypothesis is to examine the largest data sets available that have been developed in well-defined areas 


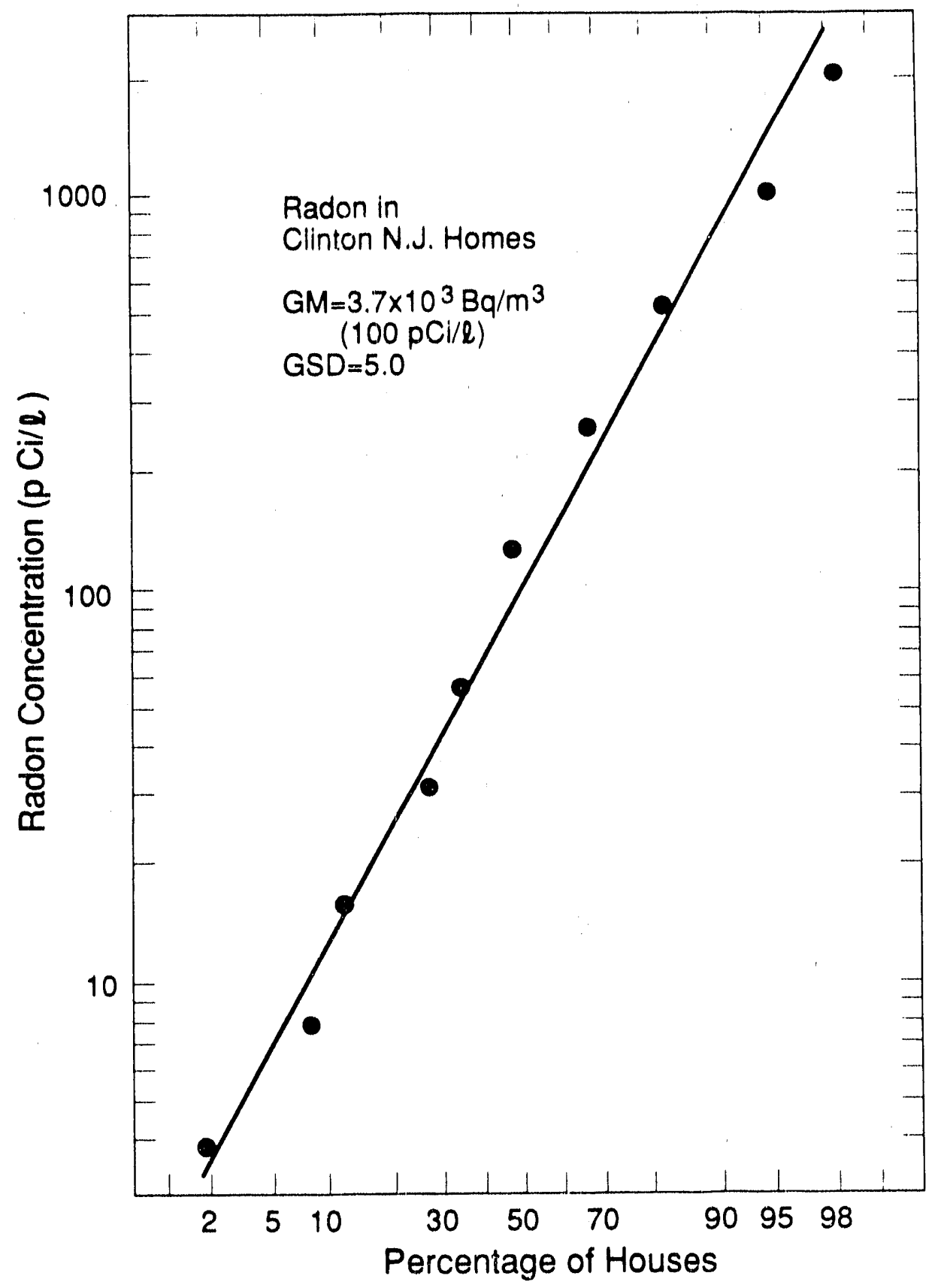

XBI $901-212$

Figure 6. Distribution of radon concentrations in a neighborhood of Clinton, New Jersey.

Data are from short-term monitoring, using charcoal collectors, in approximately March of 1986 (Osborne 1987). 103 houses were monitored. 
and with well-defined protocols. Perhaps the largest such set is data from the Bonneville Power Administration's Residential Weatherization Program (BPA 1986). This program has performed tens of thousands of measurements, using etched-track detectors, in homes in the Pacific Northwest, so that for some states there are many thousands of data, permitting an unusually precise examination of the distributional form.

As an illustration, roughly 18,000 data from Washington, in the form acquired from BPA by Lawrence Berkeley Laboratory, are shown in Figure 7 . Consistent with many other observations, the cumulative probability curve rises with respect to a straight line as the concentration increases above the high-probability region (around 50\%). On the othe: hand, at extremely high concentrations, this rise moderates, indicating a more complete and complex distributional form than can be recognized from smaller numbers of measurements. The solid curve in fact is the sum of two lognormal distributions, a dominant one (95\%) with a $\mathrm{GM}$ of $19 \mathrm{~Bq} / \mathrm{m}^{3}(0.5 \mathrm{pCi} / 1)$, somewhat smaller than average, plus a small amount $(5 \%)$ of a lognormal function having a $\mathrm{GM}$ of $116 \mathrm{~Bq} / \mathrm{m}^{3}(3.1 \mathrm{pCi} / 1)$, six times as large as that of the dominant component, and with a GSD that is slightly larger. This sum resembles the form of the observed distribution very closely, suggesting that the distribution is, in effect, the suin of two subsets, each represented well by a lognormal function.

As noted, the question of the detailed form of the frequency distribution is related to the fact that the distribution at a large scale can be considered to be the sum of distributions for smaller areas. These local distributions vary substantially from one arca to another, as indicated by the broad studies of Nero et al. (1986) and Cohen (1986) and by many local studies, such as the screening study of the Reading Prong in Pennsylvania and the survey focused on Clinton, New Jersey, for which the resulting data sets were given in Figures 5 and 6. It should be emphasized that these data sets were all taken in the winter, in basements where they existed. Nonetheless, they are striking, considering that in both areas monitoring identified at least one house with concentrations on the order of $100,000 \mathrm{~Bq} / \mathrm{m}^{3}$, and in the Clinton neighborhood studies, half the houses had concentrations exceeding 7500 $\mathrm{Bq} / \mathrm{m}^{3}$.

The EPA/state surveys discussed in the previous section also demonstrate the strong variability with location. Ironically, the EPA data released in 1988 (USEPA 1988) included identification of an area "similar in severity to the Reading Prong," i.e., Minnesota and North Dakota. It was, in fact, in the Red River Valley, at the border between these states, that both Nero et al. (1986) and Cohen (1986) found the highest U.S. concentrations to occur.

Another survey of interest because it also indicates an area with concentrations comparable to those in the Reading Prong and the Red River Valley, or other parts of Minnesota and North Dakota, was performed as part of studies in the Pacific Northwest (Turk et al. 1989a). There homes in the Spokane River Valley were found to have a distribution with a $\mathrm{GM}$ of $240 \mathrm{~Bq} / \mathrm{m}^{3}(6.5 \mathrm{pCi} / \mathrm{l})$ and a GSD of 3.1 ; the average concentration was $490 \mathrm{~Bq} / \mathrm{m}^{3}$ $(13.2 \mathrm{pCi} / 1)$. In contrast with the data from the Reading Prong, these data are taken predominantly in living areas (as opposed to basements), so that they exceed the values from the Reading Prong significantly, though this area represents a smaller population. In both cases, the data are taken in the heating season.

The main result of survey efforts is thus to provide a reasonably good appreciation of the concentration distribution, including the probability of houses exceeding various levels of concern. Furthermore, the substantial variability of concentrations with geographic area is evident, so that the fraction of homes above any given level of concern will vary greatly from one area to another. This is especially true of very high concentrations, but even 


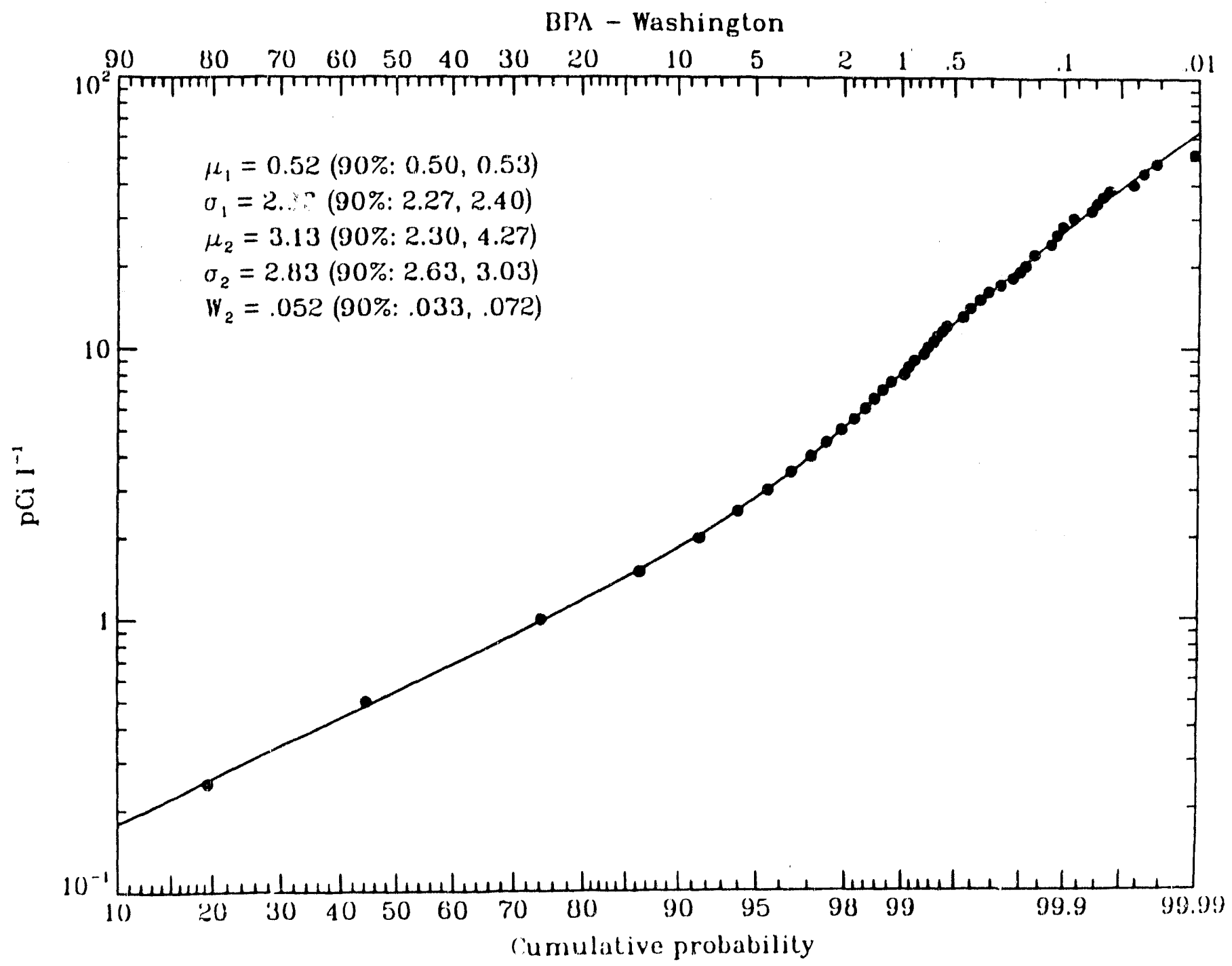

XBL 901-213

Figure 7. Distribution of radon concentrations in the state of Washington.

Approximately 18,000 data were accumulated as part of BPA's Residential Weatherization Program (BPA 1986) and are fit well by the indicated combination of lognormal distributions (where the first distribution has $95 \%$ of the weight). The parameters, $\mu$ and $\sigma$, are the GM and GSD, respectively, of the two lognormal distributions being added. $W_{2}$ is the weight of the second. 
applies to levels barely above the EPA guideline of $150 \mathrm{~Bq} / \mathrm{m}^{3}$. Thus, while very large fractions, of the order of a third, of homes in areas such as the Reading Prong or the Red and Spokane River Valleys are found to exceed an annual-average living space concentration of $150 \mathrm{~Bq} / \mathrm{m}^{3}$, in other areas the fraction is very small, no more than $1 \%$. For example, results from a survey among its employees by the Los Angeles Times indicate that only $0.7 \%$ of Los Angeles-area houses have concentrations exceeding $150 \mathrm{~Bq} / \mathrm{m}^{3}$ (Los Angeles Times 1988). These differences greatly affect the nature of strategies to limit exposures.

This is especially true in view of the fact that the distribution of human exposures is certainly very different from the distribution of concentrations, simply because people tend to live in several (or even many) houses during their lives. Their lif otime exposures thus arise from an average of the concentrations in these various dwellings. As an illustration, Figure 8 contrasts the concentration distribution resulting from the analysis of Nero et al. (1986) with the distribution of average concentrations to which people would be exposed during their lives if every person moved every seven years to a house chosen randomly from all houses in the U.S. This is an extreme assumption, but provides an effective lower bound on the breadth of the exposure distribution, whereas the concentration distribution provides an effective upper bound, i.e., the distribution of average concentrations to which people would be exposed if they all lived in the same house during their entire lifetimes. The two results are substantially different, simply because averaging across houses tends to reduce the probability of very low or very high exposures. Thus the average exposure assuming no movement results in $6 \%$ or $7 \%$ of the population experiencing lifetime average concentrations exceeding the EPA guideline, while the assumption of uniform random movement results in only $0.6 \%$ of the population experiencing such average concentrations. These limits bound the distribution of actual exposures, which we can speculate is more likely to have on the order of $2 \%$ of people exceeding the EPA guideline during their lifetimes. This is, of course, far smaller than the impression one would obtain from press reports, which suggest that 20 or $30 \%$ of the population are receiving exposures of this magnitude. The difference between the concentration and exposure distributions is likely to be even more drastic when one considers higher levels of concern, e.g., $740 \mathrm{~Bq} / \mathrm{m}^{3}(20$ $\mathrm{pCi} / 1)$.

\section{Residential concentrations in other countries}

Substantial radon monitoring efforts have also occurred in the countries other than the United States, as summarized in Table 2. One of the earliest large surveys of indoor radon concentrations was conducted in Canada, where approximate information on indoor levels has been obtained by performing grab-sample measurements in a large number $(9,999)$ of homes (McGregor et al. 1980). Rather than being a representative sampling, homes were selected from 14 of Canada's largest cities, therefore directly representing a large portion of the housing stock, but not the housing of Canada as a whole. The geometric mean EEDC varied from 3 to $13 \mathrm{~Bq} / \mathrm{m}^{3}$ among these cities, the median being $7 \mathrm{~Bq} / \mathrm{m}^{3}$. The median GSD was about 2.7. These results cannot, however, be thought of as representing the living space of these homes, since the preferred sampling site was chosen to be the basement and monitoring was done in summer. (The ${ }^{222} \mathrm{Rn}$ concentrations were also measured, but are suspect in view of the very large GSD obtained, having a median of 4.0 among the 14 cities, and the fact that measurements were performed in basements.)

Significant efforts to determine indoor radon concentrations have been conducted in numerous European countries. In several of these, efforts have been designed to provide representative information on concentrations in the housing stock as a whole. The results 


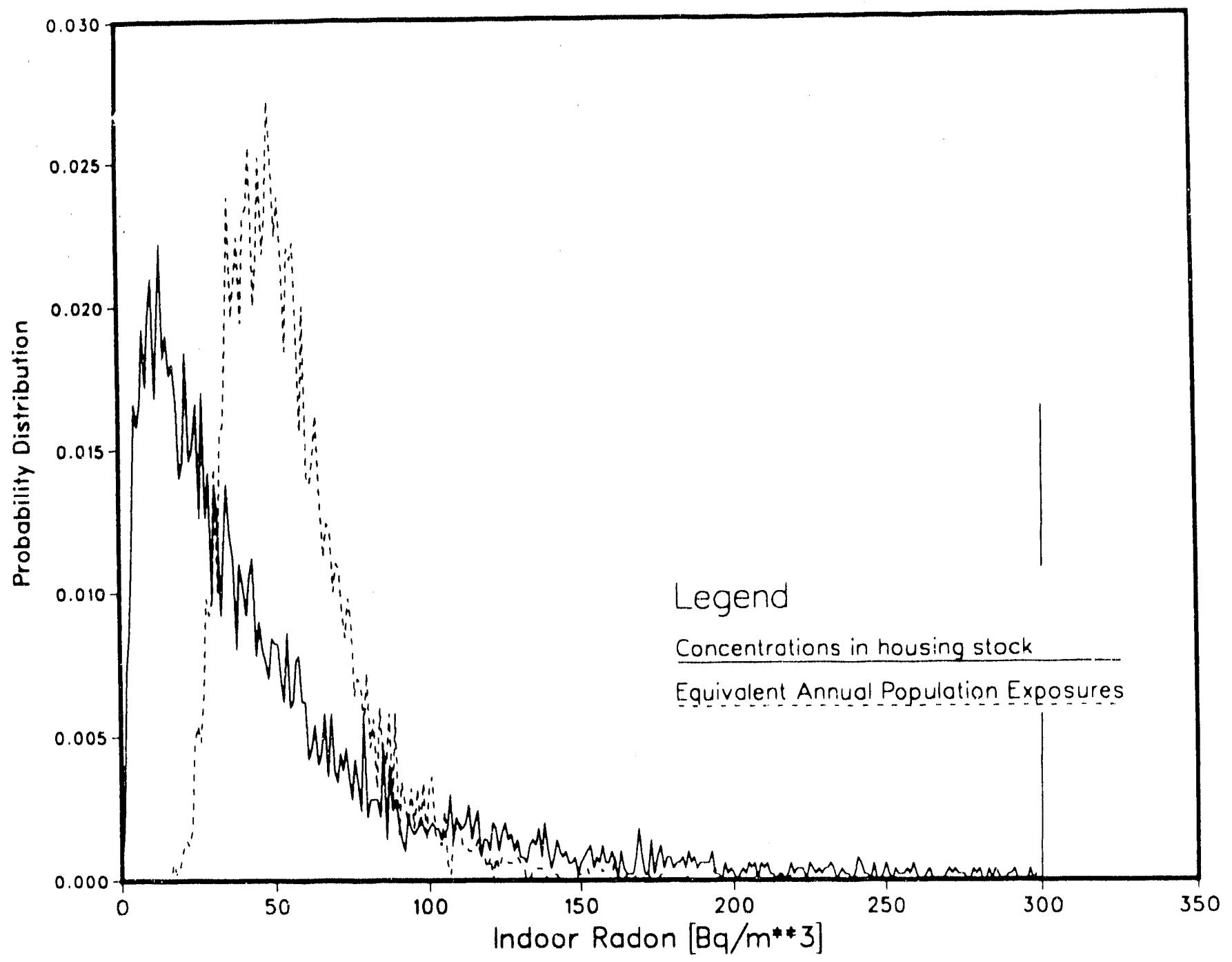

XBI $901-215$

Figure 8. Comparison of the distributions of indoor concentrations and lifetime exposures.

The solid curve is a concentration distribution (based on Nero et al. 1986) with $\mathrm{GM}=33 \mathrm{~Bq} / \mathrm{m}^{3}$ and GSD $=2.8$. The dashed is calculated using Monte Carlo sampling from the concentration distribution, assuming everyone moves to a randomly selected house every 7 years. (The simulation is for 5000 lives, each of 82 years.) 
from studies designed more or less to meet this objective differ to a significant, but understandable, degree from one country to another.

Sweden, a principal in inciting the wide interest in radon, has conducted a country-wide survey, utilizing two-week passive ${ }^{222} \mathrm{Rn}$ measurements in some 500 homes built before 1975 (Swedjemark and Mjones, 1984a). The results averaged $122 \mathrm{~Bq} / \mathrm{m}^{3}\left(\mathrm{GM} 69 \mathrm{~Bq} / \mathrm{m}^{3}\right.$ ) for 315 detached houses and $85 \mathrm{~Bq} / \mathrm{m}^{3}\left(\mathrm{GM} 53 \mathrm{~Bq} / \mathrm{m}^{3}\right)$ for 191 apartments. The distributions are approximately lognormal, with $10 \%$ of the results over $266 \mathrm{~Bq} / \mathrm{m}^{3}$ (detached) or $187 \mathrm{~Bq} / \mathrm{m}^{3}$ (apartments). About $2 \%$ of detached homes and $0.5 \%$ of apartments are estimated to have ${ }^{222} \mathrm{Rn}$ concentrations exceeding $800 \mathrm{~Bq} / \mathrm{m}^{3}$ (comparable to the Swedish remedial action level of $400 \mathrm{~Bq} / \mathrm{m}^{3}$ EEDC, assuming a typical equilibrium factor of 0.5). A supplementary study of detached houses built since 1975 yielded a GM similar to that for the larger study but with a smaller fraction at high concentrations (Swedjemark and Mjones, 1984b).

Results from measurements in about 4500 homes in Finland (not randomly sampled) yields an $\mathrm{AM}$ of about $90 \mathrm{~Bq} / \mathrm{m}^{3}$, with about $4 \%$ and $1.4 \%$ exceeding 400 and $800 \mathrm{~Bq} / \mathrm{m}^{3}$, respectively, similar to the Swedish single-family results (Castren et al. 1987); a strong geographical dependence has been observed in Finland. Results from a national survey in Denmark show somewhat lower concentrations in that country, e.g., average concentrations in single-family dwellings and apartments of 68 and $19 \mathrm{~Bq} / \mathrm{m}^{3}$, respectively (Ulbak et al. 1988). Less than $2 \%$ of houses are estimated to have annual-average concentrations exceeding $200 \mathrm{~Bq} / \mathrm{m}^{3}$. Results from 1500 homes in Norway (selected to examine geographical variability) suggest an $A M$ of 80 to $100 \mathrm{~Bq} / \mathrm{m}^{3}$, with about $1 \%$ exceeding 800 $\mathrm{Bq} / \mathrm{m}^{3}$ (Stranden 1987); an equilibrium factor if 0.5 appears to be representative.

Results from a survey of about 6000 homes in the Federal Republic of Germany using etched-track ${ }^{222} \mathrm{Rn}$ detectors yield a GM of $40 \mathrm{~Bq} / \mathrm{m}^{3}$ and GSD of 1.8 (Schmeir and Wicke 1985). In spite of the fact that a larger number appeared at higher concentrations than indicated by these lognormal parameters, only $10(0.2 \%)$ of the homes sampled had concentrations exceeding $500 \mathrm{~Bq} / \mathrm{m}^{3}$. Other results from the FRG indicate an equilibrium factor of 0.3 indoors and 0.4 outdoors (Keller and Folkerts 1984).

A survey mounted in the United Kingdom has measured ${ }^{222} \mathrm{Rn}$ concentrations in about 2000 homes, yielding a GM of $15 \mathrm{~Bq} / \mathrm{m}^{3}$ and GSD of 2.6 for living areas $\left(11 \mathrm{~Bq} / \mathrm{m}^{3}\right.$ and 2.5 for bedrooms). The ratio of summer to winter concentrations was found to be 0.51 in living areas and 0.53 in bedrooms. Concentrations substantially higher than these overall values have bee. iound in specific areas, such as Cornwall (Green et al. 1985). A national survey of about 1000 homes in the Republic of Ireland gave an average of $58 \mathrm{~Bq} / \mathrm{m}^{3}$, with about $2 \%$ of homes having concentrations exceeding $400 \mathrm{~Bq} / \mathrm{m}^{3}$ (McLaughlin and Wasiolek 1988).

Measurement of ${ }^{222} \mathrm{Rn}$ concentrations in about 1000 dwellings in the Netherlands gave a distribution well represented (except for an excess of high concentrations) by a lognormal function with GM $24 \mathrm{~Bq} / \mathrm{m}^{3}$ and GSD 1.6 (Put and de Meijer 1984).

In Europe, it is also important to realize that many of the countries mentioned have identified areas or portions of the housing stock with exceptionally high concentrations. As noted earlier, it was housing with concretes using alum shale as aggregate that drew initial attention to indoor radon as a problem, but more recently radon from the ground has been identified as the primary source in Swedish housing, and this varies substantially from one area to another. In Finland, a study on the sourh coast found averages in the 31 locales surveyed to range from 95 to $1200 \mathrm{~Bq} / \mathrm{m}^{3}$, with the total sample averaging $370 \mathrm{~Bq} / \mathrm{m}^{3}$ and $12 \%$ of houses excepding $800 \mathrm{Bg} / \mathrm{m}^{3}$ (Castren et a!. 1985) - considerably higher than the 
national parameters mentioned above. As a final example, the average from Cornwall was $390 \mathrm{~Bq} / \mathrm{m}^{3}$, fifteen times the national average for the United Kingdom (Green et al. 1985).

More limited information is available from a number of other countries. Except for special local areas, none of the results exceed the distribution found for the Scandinavian countries. And some approach the considerably lower levels characteristic of the outdoors, with average concentrations in the vicinity of $10 \mathrm{~Bq} / \mathrm{m}^{3}$.

\section{E. Other Buildings}

Relatively few data are available - particularly in published form - on radon concentrations in buildings other than residences. However, the same range of radon sources contributing to residential radon also contribute - to a greater or lesser degree - to levels in other buildings. Hence, it is to be expected that the minimum concentration observed in such buildings is the outdoor level - averaging about $10 \mathrm{~Bq} / \mathrm{m}^{3}$ of ${ }^{222} \mathrm{Rn}$, as indicated in Appendix C - but that higher levels, sometimes even into the range of those in residences, should be the norm. To the extent that structures such as hospitals or of fice buildings have a larger number of stories and higher ventilation rates than residences, we would expect lower concentrations.

Only a small number of measurements in other buildings - whether of fices, stores, hospitals, o" schools - have actually been performed, and virtually no statistically meaningful data have been developed. It is, nonetheless, worth mentioning a few substantial studies from the United States as examples. One of these was conducted in the Pittsburgh, Pennsylvania area, using grab-sample measurements taken during occupancy (an important issue if ventilation systems are turned off during unoccupied hours) in schools, stores, and other public and commercial buildings (Cohen et al. 1984). Average concentrations for various groups of buildings, many including on the order of a hundred measurements, were in the vicinity of $15-20 \mathrm{~Bq} / \mathrm{m}^{3}$, slightly higher than the outdoor concentrations measured (about $10 \mathrm{~Bq} / \mathrm{m}^{3}$ ), and much less than the annual-average concentrations found in a local survey of residential levels, averaging about $96 \mathrm{~Bq} / \mathrm{m}^{3}$. A survey of 38 office buildings in the Pacific Northwestern United States found concentrations to average 11,26 , and 44 $\mathrm{Bq} / \mathrm{m}^{3}$ for the three cities included (Turk et al. 1986). These results, obtained with integrating samplers, are somewhat lower than comparable residential concentrations in the respective areas.

Only scant information has been developed for other buildings in recent years. The notable exception is that of schools, for which the EPA recently released preliminary information on school-room concentrations during a news conference during April 1989 (USEPA 1989a), during which they recommended that schools monitor every room that is used frequently on the basement or ground-floor levels. Of approximately 3000 classrooms monitored (in 16 states) in gathering this preliminary information, $10 \%$ of the rooms tested had concentrations exceeding $150 \mathrm{~Bq} / \mathrm{m}^{3}$, and $3 \%$ gave results exceeding $740 \mathrm{~Bq} / \mathrm{m}^{3}$. These results were based on two-day charcoal collection, but other details of the monitoring protocol were not given, a critical question for schools, since these generally have mechanical ventilation systems that are of ten turned off outside of school operating hours. It is evident from other data that the EPA results cannot be used to draw general conclusions. For example, a survey of hundreds of school rooms in California found none to exceed $150 \mathrm{~Bq} / \mathrm{m}^{3}$ (California Department of Health Services 1989). 


\section{FACTORS AFFECTING INDOOR CONCENTRATIONS}

The concentration of radon in homes and other buildings varies substantially from one area to another, from one structure to another, and even within the same structure. Likewise, in the same building there is of ten a substantial variation with time on various temporal scales, i.e., season to season, week to week, and on a daily or hourly basis. Understanding the nature and origin of such variability is important as a basis for evaluating the scope of the radon problem (and indeed simply for interpreting monitoring data) and for formulating effective strategies for control. We first examine the dependence of concentration on time, and to a lesser extent on location, within the same structure. We then examine the sources and transport of radon, as a basis for understanding where high concentrations are likely to occur and for designing effective techniques for reducing the rate of entry of radon into the indoor atmosphere.

\section{A. Temporal and Spatial Dependence}

The previous section reviewed U.S. monitoring data, indicating not only the variability of indoor concentrations from one area to another, but also the critical dependence of concentration measurements on the time period and location of monitoring. The question of monitoring techniques and protocols is the subject of a later section, but we here review briefly some of the primary information on temporal and spatial dependencies, both to understand better the interpretation of monitoring data and as a further indication of the importance of differences in sources and transport processes in determining the indoor concentration.

Numerous measurements of radon in dwellings have indicated the substantial variation with time of ${ }^{222} \mathrm{Rn}$ and its decay products. This variability occurs with time of day, weather conditions, or season. Without ascribing any cause to this variability - which is certainly related to environmental or operational parameters affecting radon entry or removal (or behavior of the decay products) - we here indicate the nature of time variability by example.

One of the earlier examples of short-term variability was obtained using continuous ${ }^{222} \mathrm{Rn}$ monitors in a New Jersey house, measuring basement and upper floor concentrations over a period of weeks (Spitz et al. 1980). A significant diurnal dependence was observed, consistent with later measurements, which however sometimes showed a stronger difference between maxima and minima.

Subsequent work has examined the dependence of concentrations on various factors, directly measuring not only the concentration, but also source parameters and environmental factors affecting radon entry and ventilation rates (Nazaroff et al. 1985). An example of such realtime measurements is shown in Figure 9. Detailed examination of such correlations is important for understanding radon entry and removal, as discussed later in Section $B$.

More recent studies of radon entry have provided further information on the variability of indoor concentrations over different time scales. For example, Figures 10 and 11 show data from one of 14 New Jersey houses in which measurements examining the effectiveness of control techniques were performed over a period of approximately one year. Figure 10 shows data in which short-term variations can be seen, while Figure 11 shows results from the same house after averaging over 3 -day periods. In either case, substantial variability is evident.

It is also known that substantial seasonal variability occurs, as is of ten seen between winter and year-long measurements. For example, Table 4 shows a compa-ison of winter and annualaverage results from four different studies from Nero et al. (1986), giving the average and lognormal parameters. In these studies, the average of measured annual-average concentrations 


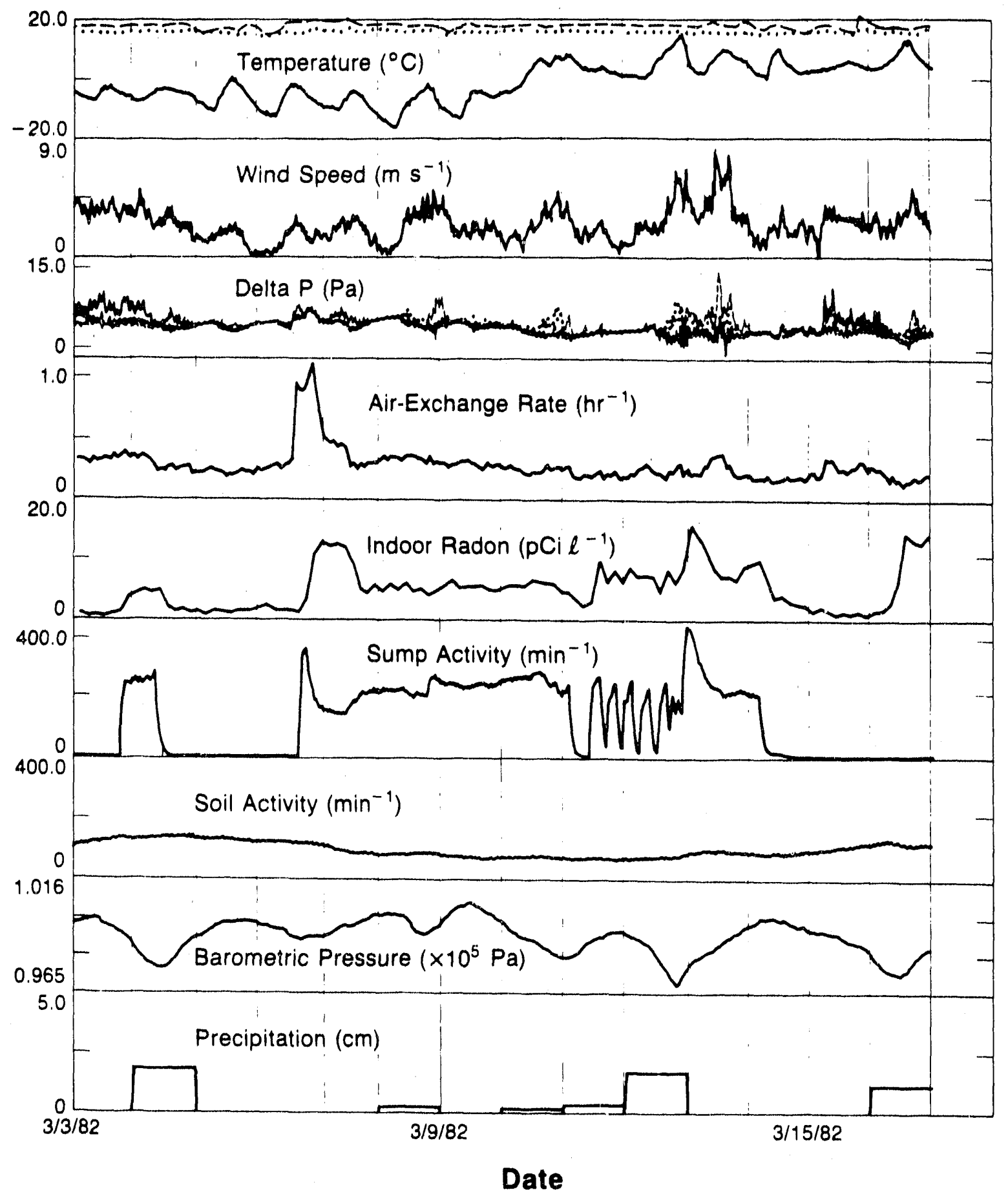

XBL $839-3147$

Figure 9. Variability of ${ }^{222} \mathrm{Rn}$ concentration, ventila!ion rate, and envirunmental parameters in a house in a basement.

These data were accumulated over a two-week period during a several-month experiment examining the dependence of radon entry on environmental factors (Nazaroff et al. 1985). 


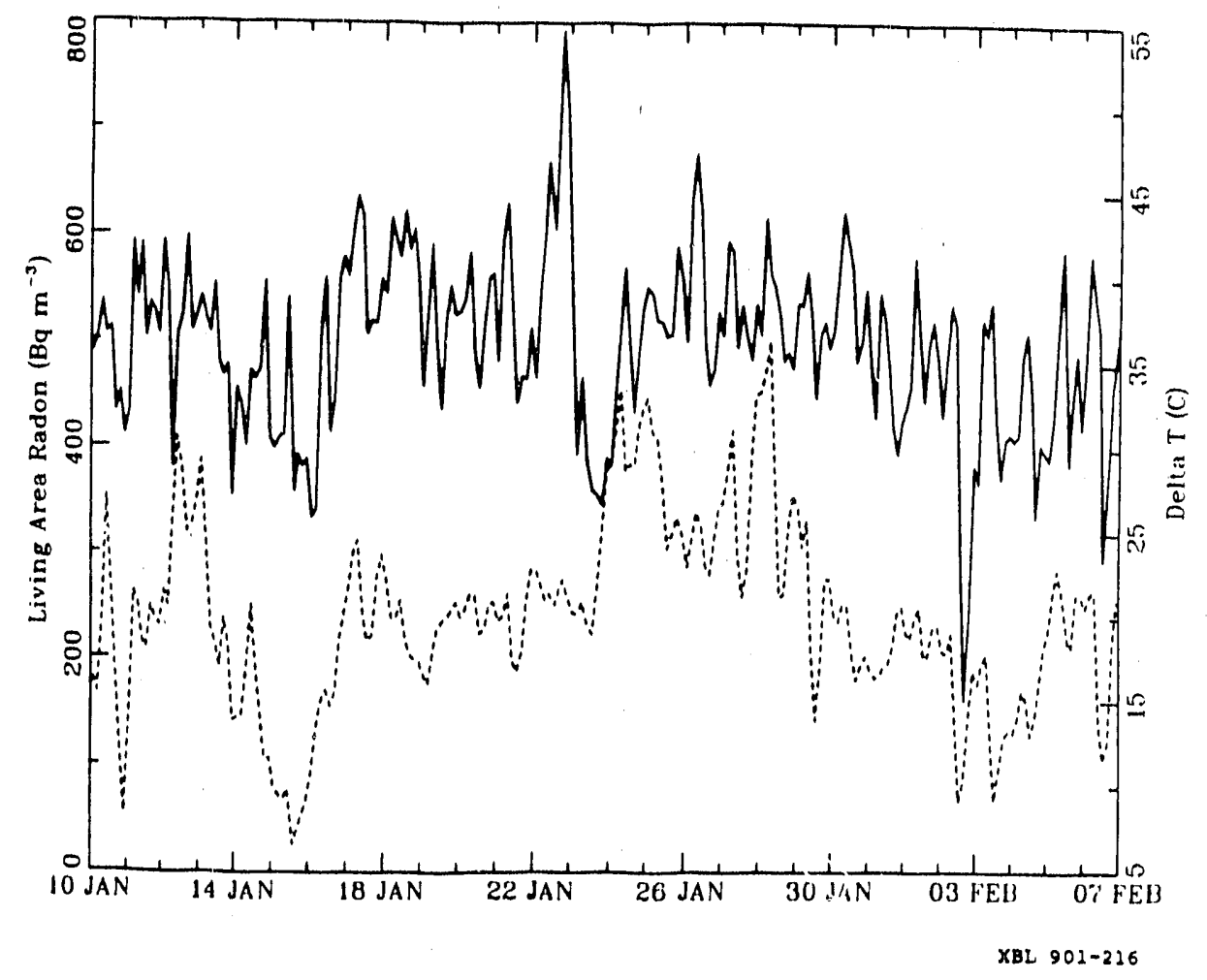

Figure 10. Radon concentration in the living space as a function of time.

Data are from a house, studied in New Jersey (cf. Revzan 1989). Also shown (dashed line) is the temperature difference between indoors and outdoors.

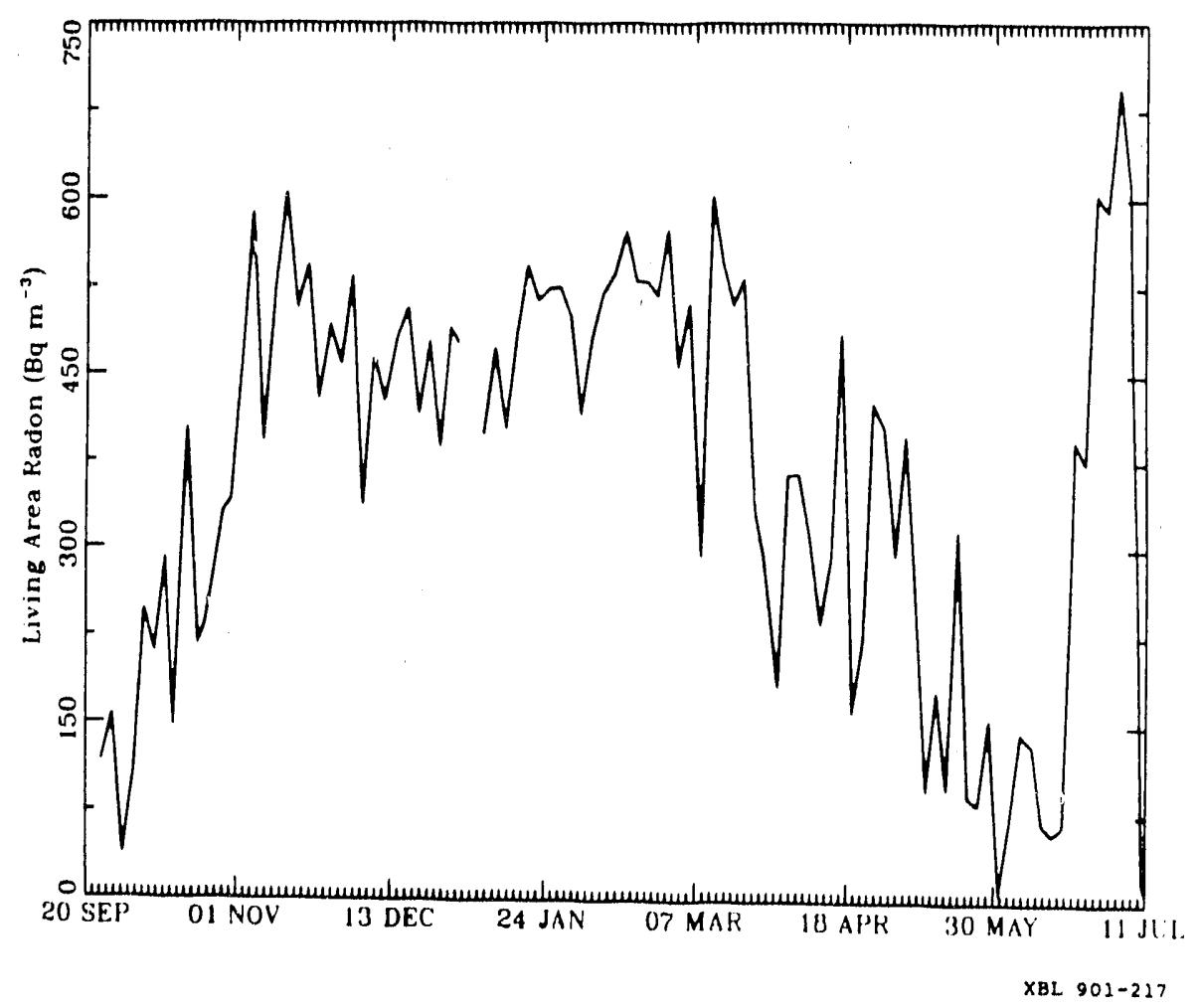

Figure 11. Radon concentration in the living space as a function of time, averaged over three-day
periods.

Data are from the same house as in Figure 10. 
is less than the average winter values (although the difference is modest in the case of Maine). If we calculate the ratio of annual-average concentrations to winter concentrations, this is found to average 0.72 . This difference has important implications for the potential interpretation of the large-scale data sets acquired primarily since 1985, discussed previously. Equally significant, in this case for assessing the prevalence of higher-than-average concentrations, is that the width of the annual-average distribution is also smaller, as given by the GSD. Assuming the GSD was the same, and that the entire distribution was merely scaled in proportion to the average in going from winter to annual-average, could thus overestimate the fraction of homes having concentrations above levels of concern such as $150 \mathrm{~Bq} / \mathrm{m}^{3}$ and $740 \mathrm{~Bq} / \mathrm{m}^{3}$. In a more recent study in Colorado, Borak et al. (1989) found - using etchedtrack detectors - the ratio of measured annual concentration to the average of summer and winter measurement results to have a mean of $1.0 \pm 0.3$. On the other hand, the GSDs for winter and summer were similar to that for year-long measurements.

The New York State survey (Hartwell et al. 1987) gives an indication of the effects of seasonal differences, based on a statistical sample. This effort gave an average of $41 \mathrm{~Bq} / \mathrm{m}^{3}(1.1 \mathrm{pCi} / 1)$ for year-long measurements in the living space, in contrast to $52 \mathrm{~Bq} / \mathrm{m}^{3} \quad(1.4 \mathrm{pCi} / \mathrm{l})$ for 2 month winter measurements. This yields a ratio of 0.79 , in the range from Nero et al. (1986). Percentages greater than $148 \mathrm{~Bq} / \mathrm{m}^{3}(4 \mathrm{pCi} / \mathrm{l})$ were $4.2 \%$ and $5.0 \%$ for year-long and winter measurements, rtspectively, showing only a slight difference. The results of Borak et al. (1989) show a greater difference, i.e., a winter to annual ratio of approximately 0.65 and percentages greater than $150 \mathrm{~Bq} / \mathrm{m}^{3}$ of $50 \%$ for winter and $20 \%$ for annual measurements.

Turning to spatial differences, the New York State study found a substantial difference between basement and living-space concentrations, with basement values averaging $100 \mathrm{~Bq} / \mathrm{m}^{3}$ $(2.7 \mathrm{pCi} / 1)$, based on year-long measurements, approximately 2.5 times the living-space average. This factor is generally consistent with other information. For example, Alter and Oswald (1987) found a ratio of 2.0, as noted above, although this is generally for shorter-term measurements. And Cohen (1989) finds an average in basements of $249 \mathrm{~Bq} / \mathrm{m}^{3}(6.7 \mathrm{pCi} / \mathrm{l})$, a factor of 2.0 times the average from living-space measurements. The New York ratio may be larger than these because it is a comparison of year-long measurements and because, whereas the living-space value tends to change substantially between winter and summer, basement values are thought to be more stable. Thus, for example, the ratio basement (year-long) to living-space (winter) measurements is 1.9 , quite close to the other results cited. Finally, there is significant evidence that, for houses with higher than average concentrations, basement concentrations may exceed those in living areas by more than a factor of 2 , an example being the factor of 5 differential for higher-concentration houses, found in NJSDH 1989.

\section{B. Sources and Transport Processes}

Radon arises from trace concentrations of radium in the earth's crust, and indoor concentrations depend on access of this radon to building interiors. Radon can enter directly from soil or rock, via utilities such as water (and, in principle, natural gas) that carry radon, or from crustal materials that are incorporated into the building structure in the form of concrete, rock, and brick. The relative importance of these pathways depends on the circumstances, but it has become clear that the first - direct ingress from the soil - ordinarily dominates the higher indoor concentrations that have been observed in homes.

Indications of this arose in early investigations of radon in U.S. houses, when it was found that measurements of radon emanating from structural materials could not account for observed indoor concentrations, based un estimates of the air exchange rate (George and Breslin 1980). Moreover, practical experience in reducing concentrations in the Canadian mining communities made it clear that the major entry route was through the house understructure, at least in the houses in which remedial measures were attempted (Scott 1988). 


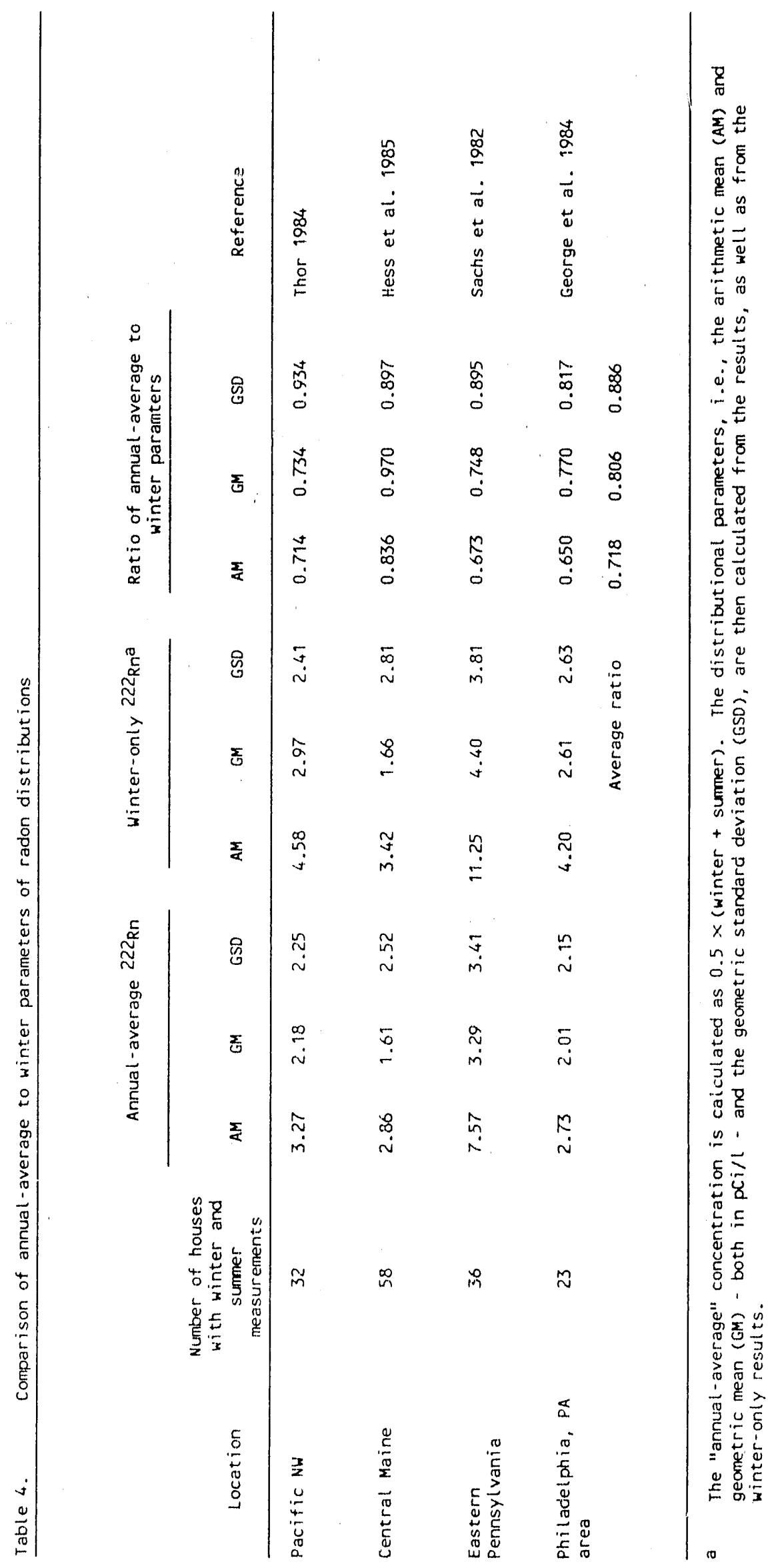


A clearer picture emerged with availability of information on the distribution of entry rates inferred from direct measurements of radon concentration and ventilation rate (such as the data shown in Figure 3). Figure 12 shows such entry-rate distributions from various countries, as well as indicating the potential contribution of various sources. Although building materials were first suspected as the major source in the U.S., based on the experience in Europe (e.g., Hildingson 1982), the initial U.S. results strongly suggested that the soil must be the major source (Nero et al. 1983). Understanding how the rate of radon entry could be approximately equal to the unimpeded flux from the ground (i.e., in the absence of the house) has been a major focus of research on radon entry, in both North America and Europe.

This section discusses briefly the current state of knowledge of radon sources and transport. Far more comprehensive treatments are given in Nazaroff et al. (1988), Stranden (1988), and Nazaroff et al. $1987 \mathrm{~b}$.

\section{Soil and Building Materials}

Understanding the radon mass balance for a building requires specific consideration of the various sources. As indicated in Figure 12, a median (or GM) entry rate for U.S. single-family houses appears to be in the vicinity of $20 \mathrm{~Bq} \mathrm{~m}^{-3} \mathrm{~h}^{-1}\left(0.5 \mathrm{pCi}^{-1} \mathrm{~h}^{-1}\right)$. Based on emanation rate measurements from U.S. concretes (Ingersoll 1983), one might expect emissions from this source to account for a median of about 2 to $3 \mathrm{~Bq} \mathrm{~m} \mathrm{~m}^{-3} \mathrm{~h}^{-1}$, far below the rate observed. On the other hand, the potential contribution from unattenuated soil flux, a median of about $25 \mathrm{~Bq} \mathrm{~m} \mathrm{~m}^{-3} \mathrm{~h}^{-1}$ (based on Wilkening et al. 1972), corresponds much more closely with the indoor observations. However, houses have understructures that might be expected to impede substantially the ingress of radon, at least by diffusion, the main mechanism for causing the observed fluxes from uncovered soil. As discussed in detail in Nero and Nazaroff (1984) and in Nazaroff et al. (1988), although transport via diffusion accounts well for observed fluxes from building materials and exposed soil (and could account for small fluxes from the soil through some understructure materials, such as concrete), diffusion cannot account for the total entry rates observed in single-family houses. Another mechanism must account for the efficiency with which radon from soil enters such homes. It appears that this mechanism is bulk flow of soil gas driven by small pressure differences between the lower part of the house interior and the outdoors.

As discussed in Appendix B on ventilation rates, pressure differences of only a few pascal i.e., on the order of only a ten thousandth of an atmosphere - arise from winds and indooroutdoor temperature differences and are the cause of ventilation in homes during seasons when the windows are closed. These same pressure differences can, in principle, drive the small flows of soil gas needed to account for the observed rate of radon entry into homes: soil gas contains enough radon that, on the average, unly $0.1 \%$ of infiltrating air would have to be drawn from the soil to account for observed indoor concentrations (Nero and Nazaroff 1984).

Work during the 1980 s has been aimed at characterizing directly and systematically the potential for pressure differences to cause entry of radon via soil gas, probably through imperfections and penetrations in the house understructure that permit passage of the relatively small amount of soil gas required. The prototype of such a work was a study of radon entry in a single family house with a basement (see Figure 9) that analyzed the entry rate versus the ventilation rate, measured over a period of months, and concluded that entry could usefully be represented by a sum of two components: one - the smaller - independent of ventilation rate, much as diffusion would be, and a larger term proportional to ventilation rate, as pressuredriven flow might be (Nazaroff et al. 1985). Moreover, the observed pressure and soil parameters appeared consistent with the soil-gas flow rate that was implied by the measured concentrations and ventilation rates. 


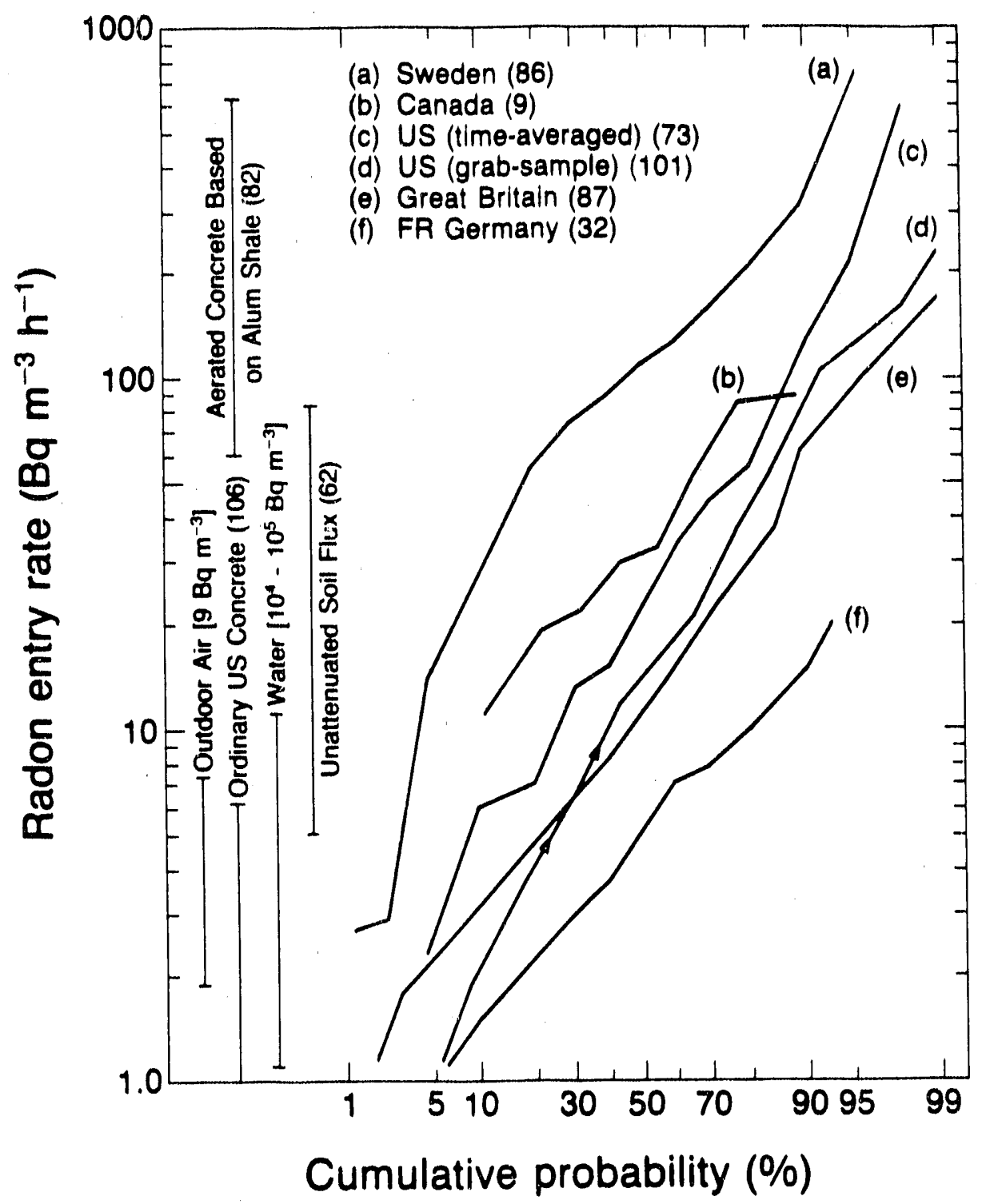

XOL $842 \cdot 10053$

Figure 12. Cumulative frequency distributions of radon entry rates in dwellings.

The entry rate is determined from the product of simultaneously measured radon concentration and ventilation rate. The number of residences in each sample is indicated in parentheses. The references from which these results are taken are: (a) Hildingson (1982); (b) Smith (1979); (c) Nazaroff et al. (1981), Nazaroff et al. (1985), Nazaroff and Doyle (1985), Doyle et al. (1984), Nazaroff et al. (1983), Nazaroff (1983b); (d) Nero et al. (1983); (e) Cliff (1978); and (f) Wicke (1979). The bars at the left indicate the range of contributions expected from a variety of sources, with assumptions indicated in brackets. For each source, we have assumed a single-story house of wood-frame construction with a $0.2 \mathrm{~m}$-thick concrete-slab floor. The floor area and ceiling height are assumed to be $100 \mathrm{~m}^{2}$ and $2.4 \mathrm{~m}$, respectively; water usage is assumed to be $1.2 \mathrm{~m}^{3}$ per day, with a use-weighted transfer efficiency for radon to air of 0.55 ; and the ventilation rate is assumed to be in the range $0.2-0.8 \mathrm{~h}^{-1}$. References for estimates of source contributions are: outdoor air (Gesell 1983); U.S. concrete (Ingersoll 1983); alum-shale concrete (United Nations Scientific Committee on the Effects of Atomic Radiation 1982); water (Nero and Nazaroff 1984); and soil flux (Wilkening et al. 1972). (Figure taken from Nazaroff and Nero 1984.) 
Although other research has sometimes suggested that diffusion provides the dominant contribution to entry rate (e.g., Holub et al. 1985), such results usually appear questionable (as indicated, for example, by the follow-on correspondence given with Holub et al. 1985), and the preponderance of other work (e.g., Bruno 1983 and Akerblom et al. 1984) indicates the importance of soil-gas flow.

This is also indicated by work following the initial studies just mentioned. Perhaps most significant, experiments have directly observed, in houses with basements, the underground depressurization implied by this picture, and have monitored underground soil-gas movement by'injecting and monitoring tracers (Nazaroff et al. 1987). These observations have been confirmed in subsequent work, including a different kind of experiment, i.e., practical experience with remedial measures in eastern Pennsylvania (Henschel and Scott 1986), the Spokane River Valley (Turk et al, 1989a,b), and other areas with high radon concentrations, using techniques of the kind discussed in Scott (1988). See the more detailed discussion of control techniques in Section III.D. Such work is confirming and providing new information on the flow of radon-bearing air through house understructures. It is interesting to note that these results may also have significant implications for entry of other pollutants from the soil (Nazaroff et al. 1987, Kliest et al. 1989, Garbesi and Sextro 1989).

Finally, the fundamental picture of radon entry suggested by this work has been complemented by increasingly sophisticated modeling of radon entry, beginning with simulations described in DSMA (1983). Later, analytical representations of entry and removal were found useful by Mowris and Fisk (1988) for examining the influence of exhaust ventilation (which artificially depresses the pressure inside the structure) on indoor levels. Arvela et al. (1988) have, using similarly simple approaches, attempted to predict ndoor concentrations by modeling the manner that entry and removal are influenced by metcorological factors. Loureiro (1987) used a 3-dimensional finite-difference model to simulate radon entry assuming a constant pressure difference, permitting the soil type in the immediate vicinity of the basement to differ from that further away. In parallel, Schery et al. (1988) have continued to develop the basic formalism for transport through porous fractured media (cf. Nazaroff et al. 1988).

The next step is to extend modeling to consider 1) more heterogeneous configurations in respect to substructure and soil, 2) time-dependent effects (both short-and long-term), and 3) two-phase effects involving the interaction of water and soil gas. For example, Narasimhan et al. (1989) have begun to examine the effects of transient air flow theoretically. A more complete discussion of the objectives and and state of modeling, including its use for understanding the effectiveness of control techniques and for simulating the distribution as a function of geographic or numerical variables, is given in Section III.C.

The studies of houses with basements have given results that may also apply in large part to slab-on-grade structures, where the pressure difference generated can still draw soil gas through any penetrations in or around the slab. However, few direct measurements in such structures have been reported. A notable exception is the case of Clinton, NJ, where very high concentrations were discovered (Osborne 1987), as seen in Figure 6, and where some of the houses were wholly or partially slab-on-grade. (However, it should be noted that these homes had sub-slab heating ducts whose presence contributed substantially to radon entry.)

The other understructure type of substantial importance is the crawl space, which to some extent isolates the interior from the soil - at least in respect to pressure-driven flow between the two. Limited measurements of the transport efficiency of radon through crawl spaces yield the result that a substantial portion of the radon leaving uncovered soil manages to enter the interior, even if vents are open to permit natural ventilation of the space (Nazaroff and Doyle 1985).

In retrospect, this is not entirely surprising, since the stack effect will tend to draw infiltrating 
air in to the home from the crawl space, which can retain radon from the soil in conditions where winds are not sufficient to flush it to the outdoors via the vents. Furthermore, for structures where the vents are sealed shut, e.g., to save energy, it is conceivable that the crawl space still provides sufficient connection between the house interior and the soil that pressuredriven flow can enhance the flux from the soil above levels associated merely with diffusion; the work reported in Nazaroff and Doyle (1985) may have observed this effect. Another observation from this study is that energy conservation efforts that focus on tightening the floor above a crawl space can significantly reduce infiltration rates, while reducing radon entry a corresponding amount, as a result of which indoor concentrations are little affected. The modeling approach of Mowris and Fisk (1988), together with the basic understanding of infiltration (see Appendix B), affords direct means for assessing the effect of tightening measures to reduce either entry or air infiltration.

Thus sufficient mechanisms exist to account for the substantial amount of radon that appears to enter single-family homes from the soil, apparently without great regard to substructure type. However, this does not imply that other sources of radon are unimportant. As discussed elsewhere (Stranden 1988), it is clear that materials utilized in a building structure can contribute substantial indoor concentrations, although this is not usually the case (even for natural stone that has higher-than-average radium content). Moreover, in buildings that are relatively isolated from the ground, such as multi-story apartment buildings, indoor concentrations are expected to be lower than average - as if of ten the case in central European dwellings - and to arise primarily from the building materials and from radon in outdoor air.

\section{Water}

Probably more important than buildings materials, as a source of radon in certain parts of the housing stock, is domestic water drawn from underground sources. Surface waters have radon concentrations too small to affect indoor levels when used domestically, but ground water is in a good position to accumulate radon generated within the earth's crust. As a result, very high radon concentrations can sometimes be found in associated water supplies. For example, a survey of ${ }^{222} \mathrm{Rn}$ concentrations in well-water in Maine found a range of $7 \times 10^{2}$ to $7 \times 10^{6}$ $\mathrm{Bq} / \mathrm{m}^{3}$ (20 to $\left.180,000 \mathrm{pCi} / 1\right)$, while wells in granitic areas were found to average $8 \times 10^{6}$ $\mathrm{Bq} / \mathrm{m}^{3}$ (Hess et al. 1983); and the average concentration in water from drilled wells in Finland has been found to be $6.3 \times 10^{5} \mathrm{~Bq} / \mathrm{m}^{3}$ (Asikainen and Kahlos 1980). With normal water use, the radon entering indoor air from water with such high concentrations can be expected to be significant.

Examinations of the overall potential contribution of water-borne radon to indoor concentrations have tended to be no more sophisticated than to consider typical water use rates, house volumes, and ventilation rates, yielding a ratio of radon in air to radon in the water supply of about $10^{-4}$. However, it is possible to make a more realistic estimate of the distribution of the air-to-water ratio, using more detailed distributional information (rather than just averages) on water use rates, efficiency of radon release from domestic water used in various ways, house volumes, and ventilation rates. Such an analysis of U.S. data for singlefamily houses yields a ratio with a lognormal distribution having a GM of $0.65 \times 10^{-4}$ and a GSD of 2.88 , as discussed by Nazaroff et al. (1987b). Taken together with recently developed data on ${ }^{222} \mathrm{Rn}$ in public water supplies (Horton 1985), such a distribution permits quantitative assessment of the contribution of public water supplies to indoor radon concentrations. The result of such an assessment is that such supplies contribute an average of approximately 1 $\mathrm{Bq} / \mathrm{m}^{3}(0.03 \mathrm{pCi} / \mathrm{l})$ in the $30 \%$ of U.S. homes served by ground water, i.e., only about $2 \%$ of the average indoor concentration in U.S. single-family homes.

However, the very high water-borne concentrations that are sometimes found - particularly from private wells - must be expected to contribute much larger airborne concentrations in 
the homes affected. Using the few data that are available for the approximately $18 \%$ of the U.S. population using private wells, the airborne contribution from water for this segment of the housing stock can be estimated to average about $20 \mathrm{~Bq} / \mathrm{m}^{3}$ (Nazaroff et al. 1987b). About $10 \%$ of the houses served by these wells (totaling about $2 \%$ of the U.S. housing stock) are estimated to have indoor concentrations from water of $40 \mathrm{~Bq} / \mathrm{m}^{3}$ or more. Although these estimates for radon from private wells cannot be regarded to be reliable, they suggest that the portion of the population using private wells may be experiencing significantly higher radon exposures than average, particularly in areas with high radon activity in water.

A recent report of results of radon monitoring in water from private wells, although not from statistically designed surveys, seems to confirm these indications, although a somewhat smaller contribution from water is suggested. The results gave state-wide average concentrations in private-wel! ater ranging from about $10,000 \mathrm{~Bq} / \mathrm{m}^{3}$, indicating a contribution to indoor air averaging about $1 \mathrm{~Bq} / \mathrm{m}^{3}$ in such states, to about $500,000 \mathrm{~Bq} / \mathrm{m}^{3}$ in several states, implying an average contribution of about $50 \mathrm{~Bq} / \mathrm{m}^{3}$ (comparable to the national average) in houses with private wells in such states (Viiz 1989). The average of statewide averages was approximately $125,000 \mathrm{~Bq} / \mathrm{m}^{3}$, implying an average waterborne contribution, in houses with private wells, of $10-15 \mathrm{~Bq} / \mathrm{m}^{3}$, slightly lower than suggested above. In addition, there may be a bias toward high-concentration houses, considering the manner of distribution of the kits. Nonetheless, these results confirm the potentially significant contribution of radon from private-well water, particularly in the fraction of houses with water-borne raden concentrations that are significantly higher that the statewide averages. Such results also suggest that, overall, waterborne radon may contribute about $5 \%$ of the about $50 \mathrm{~Bq} / \mathrm{m}^{3}$ average radon concentration in U.S. homes.

\section{Summary of Source Contributions}

We can thus point to the major sources of radon present in indoor environments. For singlefamily houses and other structures of one or two stories, the ground constitutes the principal source, but with a noticeable portion entering with outdoor air, i.e., on the order of $10 \mathrm{~Bq} / \mathrm{m}^{3}$ (cf. Appendix $C$ ). However, there are circumstances in which the building materials or domestic water supplies are important. In contrast, in high-rise buildings, including apartments, the ground is of lesser importance, and the amount of radium-bearing building materials per unit volume is larger, so that outdoor air and the building materials are typically the dominant contributors, although the ground and water supplies can be important in some cases. Other potential sources, such as natural gas ordinarily contribute negligible amounts by comparison (Gesell 1973, Johnson et al. 1973).

For a specific set of assumptions, characteristic of U.S. buildings, Table 5 indicates the approximate average contribution of various sources to the indoor concentration for singlefamily buildings and (to the extent data are available) for apartments. For comparison, the observed concentrations are also given. Note that, considering the uncertainties involved, the presumed contributions are consistent with the observed levels, i.e., an average of $55 \mathrm{~Bq} / \mathrm{m}^{3}$ in single-family houses and a substantially smaller concentration in high-rise apartments.

\section{Capabilities for predicting indoor concentrations}

Development of predicti e models of the indoor radon concentration is useful, and in fact, essential for several purposes. The overall (national) distribution of the average indoor radon concentration in the existing housing stock can be determined by appropriate survey efforts, but in fact much more information is required. This inciudes $i j$ the deveiopment of mucieis that represent research results in such a way as to simulate the radon concentration in a given house as a function of time in a given house (diurnal and seasonal variations), and that can be 
Table 5. Approximate Contributions of Various Sources to Observed Average Radon Concentrations. ${ }^{\mathrm{a}}$

\begin{tabular}{lcccc} 
& \multicolumn{2}{c}{$\begin{array}{c}\text { Single-Family } \\
\text { Houses }\end{array}$} & \multicolumn{2}{c}{$\begin{array}{c}\text { High-Rise } \\
\text { Apartments }\end{array}$} \\
\cline { 2 - 5 } & $\mathrm{Bq} / \mathrm{m}^{3}$ & $(\mathrm{pCi} / \mathrm{l})$ & $\mathrm{Bq} / \mathrm{m}^{3}$ & $(\mathrm{pCi} / \mathrm{l})$ \\
\hline $\begin{array}{l}\text { Soil potential (based on flux } \\
\quad \text { measurements) }\end{array}$ & 50 & $(1.5)$ & $>0$ & $(>0)$ \\
$\begin{array}{l}\text { Water (public supplies) } \\
\text { Building materialsc }\end{array}$ & 0.4 & $(0.01)$ & 0.4 & $(0.01)$ \\
Outdoor air & 2 & $(0.05)$ & 4 & $(0.1)$ \\
\hline Observed indoor concentrations & 10 & $(0.25)$ & 10 & $(0.25)$ \\
\hline
\end{tabular}

a In each case, the arithmetic mean is shown, based on entry rates (or, in the case of outdoor air, concentrations) discussed in the text and on an air exchange rate of $0.5 \mathrm{~h}^{-1}$.

b Indicated water contribution applies to $80 \%$ of U.S. population served by public supplies. Contribution may average $15 \mathrm{~Bq} / \mathrm{m}^{3}$ in homes using private wells, with even higher cuntribution in high-activity areas.

c The contribution of building materials in single-family houses corresponds to a slightly smaller geometric mean entry rate than the 2 to $3 \mathrm{~Bq} \mathrm{~m}^{3} \mathrm{~h}^{-1}$ estimated in the text because not all houses are slab-on-grade or one-story. A higher cortribution to apartment air is suggested on the presumption that, on the average, high-rise apartments have a larger amount of radium-bearing material per unit volume than do single-family homes. 
be used to evaluate the effectiveness of control techniques, as well as to interpret monitoring results, and (2) the cormulation of operational models to estimate the expected distribution in housing stock subsets, depending on the type of construction, geographic area, etc.

Predictive models are desirable for

- estimating population risk from indoor radon exposures,

- assessment of relative costs of mitigation per avoided statistical death, for various control measures, and relative to measures to reduce risks from other (non-radon) causes,

- identification of population segments at high risk, and setting in motion policies to reduce these risks,

- estimation of impacts of changes in demographic patterns, the building stock and building operation (of ten owing to energy conservation measures), changes in occupant behavior, in terms of changes in risk to the population, and the costs of control measures,

- providing guidance for conducting house-to-house surveys of indoor radon concentrations (when to measure, where to measure),

- interpreting monitoring results, and

- identifying data gaps and imperfect understanding of the physical mechanisms involved in radon generation, transport, entry and removal.

Predictive models of exposure must be based on predictive models of concentrations, folding in data on time-activity and mobility patterns of the population whose exposure is to be evaluated.

Predictive models of indoor radon concentration span a wide range of model types, both in terms of complexity and in terms of specificity. At one end of the specificity spectrum would be a model of a particular house (with or without a basement) sitting on the soil, and incorporating submodels that attempt to simulate the physical phenomena occurring in terms of soil-gas transport and entry into the house, including the subsequent behavior of radon (and perhaps its decay products) in the various zones of the house, until their removal from the indoor air by decay and ventilation (and perhaps plateout and deposition in the lung). The specific model may be simple or complex, in terms of how much of the detail is modeled, as suggested in the models referred to above (Section III.B). At the other end of the spectrum of specificity would be a predictive model that attempts to estimate the overall distribution of indoor radon concentrations in U.S. homes, on an annual-average basis. Again the level of complexity could vary, ranging from a single general distribution to a model that incorporates and indicates the dependence on climate, soil properties, regional construction styles, etc.

The two types of models described above are based on different approaches to the problem. The first model is based on modeling the physical processes involved in the house in detail and on verification of the sub-models with detailed measurements and adequate samples of intermediate data. Such models result from, and are directly associated with, the basic experimental work on radon (and decay product) behavior. The second approach is based on surveys of underlying factors - and to some extent of indoor radon concentrations - in adequately large samples of the housing stock, developing distributions to represent the entire housing stock of interest. In fact, the above two examples describe the two extreme cases; in practice, a combination of both the approaches in some judicious measure is usually adapted. 
To enable the modeling effort to fulfill the expectations described above, the sampling survey approach is inadequate for prediction of impacts of various changes in building stock and its use and operation, because the underlying understanding of the causative mechanisms is missing; on the other hand the mechanistic model, though rich in its analytical detail of the causative mechanisms, is inadequate of itself to answer questions regarding exposures and risks to the overall population because data on distribution of housing constructions, soil types, and other explanatory variables can be obtained only from surveys.

\section{House or site models}

Progress on models for individual houses (or sites) is illustrated by the work discussed in the previous section, i.e., DSMA (1983), Mowris and Fisk (1988), Loureiro (1987), and so on. A detailed predictive model of indoor radon concentrations in a house would require development and integration of the several sub-models. Figure 13 illustrates the structure of such a model for a general indoor pollutant where the indoor concentration is determined by mass balance relationships involving sources, ventilation and reactions, which - in turn - are influenced by building design, meteorology, occupant behavior and, of course, innate characteristics of the sources of each pollutant. Radon-specific aspects of such a structure are shown in Figure 14. Major components of a radon model, and an indication of our current understanding of the physical processes, include:

- By far the dominant mode of entry of radon into houses is pressure-driven flow of radon-bearing soil gas into the house, as discussed in the previous section. For modeling this phenomenon, which is the most important entry route from the viewpoint of understanding high indoor radon concentrations, at least a three component model is needed: 1) a model of how the soil moisture content, permeability and emanating fraction of radium are distributed beneath and in the vicinity of the house, 2) a model of the house-soil interface in terms of occurrence and extent of any horizontal gaps between the slab and the underlying soil or (more commonly) existence and characterization of any layer of highly permeable gravel under the slab, the extent and character of cracks and openings in the slab and basement walls to the soil, any layer of uncompacted soil near the basement walls, existence and extent of a small gap (owing to soil subsistence and shrinkage) between the basement walls and the adjacent soil, construction details of the basement slab and walls and any drainage system, and 3) a model of the physical phenomena that cause the pressure differences for driving the soil gas into the house, such as the indoor-outdoor temperature difference, operation of appliances that exhaust house air to the outside (thus contributing to the induced pressure difference), wind-induced pressures owing to its interaction with the soil and house surfaces, and the influence of barometric pressure fluctuations on the soil and house pressure fields. This structure is indicated in Figure 15.

- The radon concentration in the water used in the house will be primarily determined by whether the water supply is from a private well and the radium concentration in the soil with which the ground-water in the well has had contact. The radon that enters the house from the use of such water depends on the amount of water use and on the kind of use to which the water is put. (For example, use of this water for flushing a toilet releases about 3 times less radon per unit of water use than from taking a shower (Nazaroff et al. 1987b).

- Diffusion of radon into indoor air owing to building materials is determined by the diffusivity of radon and the radium content in the materials (Stranden 1988). Similar diffusive entry from bare soil (in the absence of a basement slab) is controlled by the radium concentration, the emanating fraction of radium, and the diffusivity of radon in the soil. 


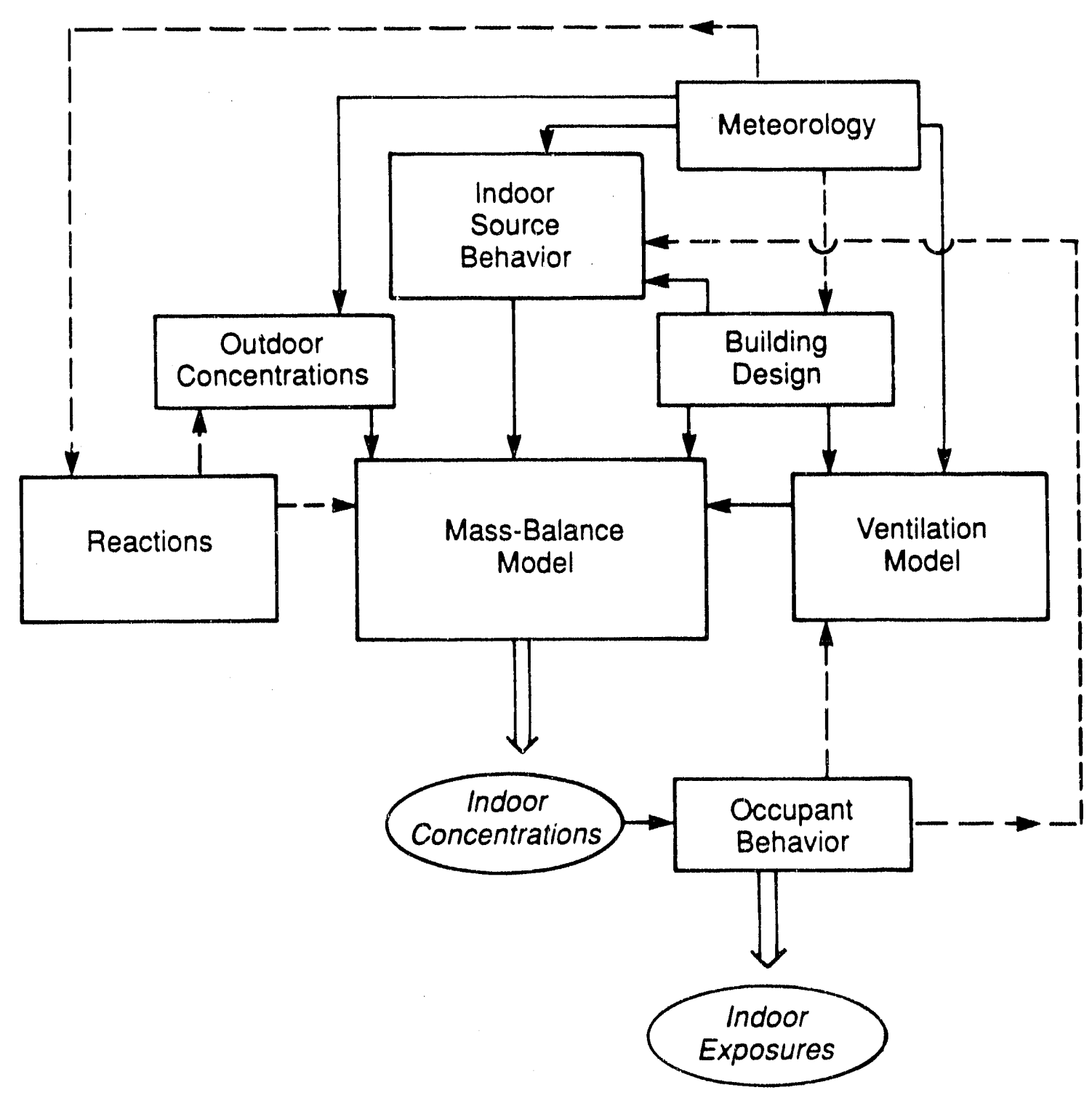

XBL $901-6216$

Figure 13. General design of a physical model for the indoor air quality in a house.

Concentrations of a specific substance are determined by competing source and removal terms as indicated and, in principle, may exhibit variability with both location and time. (Reactions may also include those with other indoor pollutants, not indicated.) 


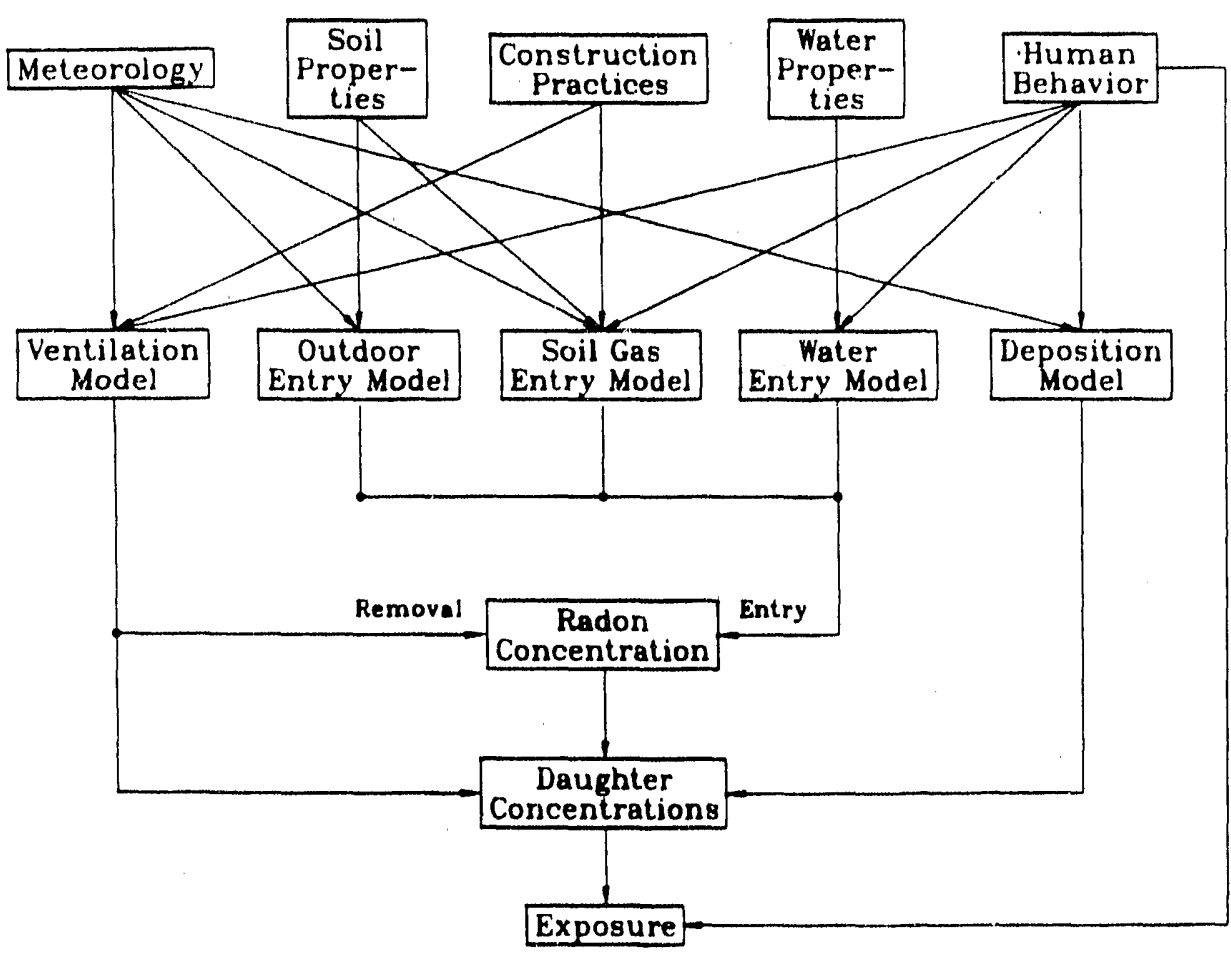

XBL $902-214$

Figure 14. Design for a physical model of the radon concentration in a house.

The indoor radon concentration may be calculated from a variety of sub-models which, in turn, utilize a variety of physical (and human) data.

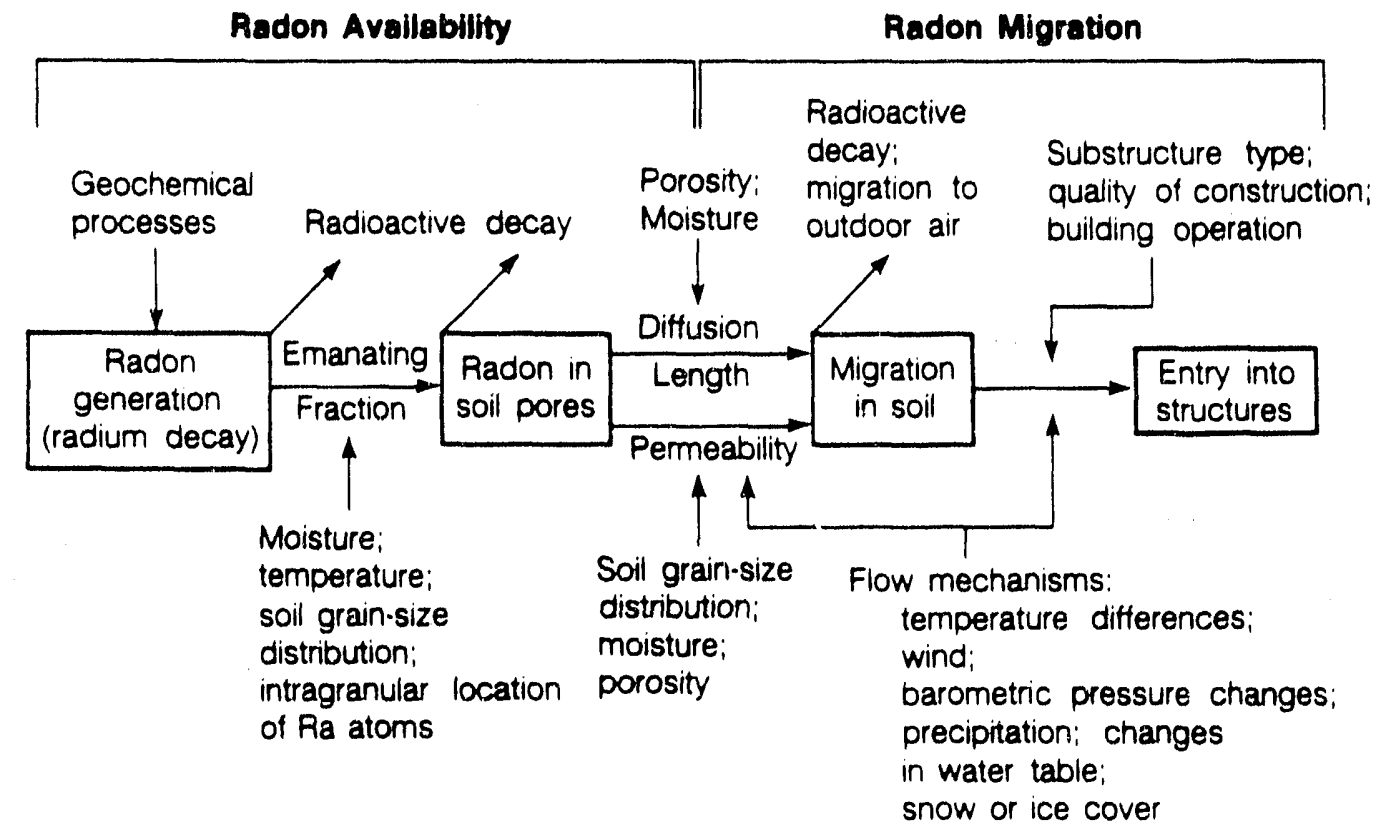

XBL8510.11933

Figure 15. Overall design for understanding or modeling the entry of radon from the soil.

Radon entry alone, if represented completely in a model, involves a complex of processes and information affecting radon generation and transport. 
- The subsequent behavior of radon that enters the house via various routes is determined by the interzonal convection and by ventilation rates in the various zones; both of these are influenced by infiltration and temperature-induced pressure differences, which also influence the pressure-driven entry rate of radon via the soil gas, so the behaviors of these sub-models are not really separable. See Appendix B for a brief discussion of ventilation and associated models.

- The behavior of decay products of radon in the indoor air further depends on the concentration and size distribution of indoor aerosol which they encounter. (This topic is treated separately in Section V.)

- Finally, the exposure of house occupants to radon decay products is influenced not only by their occupancy patterns, but also by their own operation of the house, such as thermostat settings, operation of whole-house or kitchen exhaust fans, use of aircleaners to remove indoor aerosols, activities that generate indoor aerosol, etc.

It seems likely that only large numerical models would be able to handle the simulation and integration of the detailed sub-models that is expected from the above description of a complete house model. A further difficulty is that there are considerable gaps in our present ability to describe the above phenomena mathematically. For example, the interaction of the external wind field with the soil surface and the house, which influences the soil-gas entry into the house (and may influence radon concentrations in the soil gas itself), is poorly understood and hence difficult to model.

Furthermore, there are often practical problems in obtaining input data for some phenomena that we believe we understand and can model adequately. For example, even though sophisticated and detailed models of three dimensional, soil-gas transport driven by an externally imposed pressure field exist (Loureiro 1987, Narasimhan et al. 1989), it is not clear how, in a practical situation, one would go about obtaining a "sufficiently" detailed and accurate map of distribution of relative permeability and emanating fraction of radium in the soil near and beneath the house to enable one to predict with confidence the behavior of the entry sub-model. Often the test of "sufficiently" adequate data is met only by comparing prediction with experiment, and obtaining more and more data till the predictions are consistent with observations. This problem also applies to the input data limitation on interzone convection, and the soil-house interface.

On the other hand, the more complete development of such models does offer a framework for representing our increasing understanding of the entry and removal of radon, and hence is a necessary feature of research in this area. Furthermore, as this understanding becomes more firm, it is hoped that simplified forms of such models can serve effectively in key applications. The most obvious is to improve the design of control techniques appropriate ti various situations. The more subtle is to use the models as a basis for interpreting monitoriag data and, indeed, as a basis for the housing stock models discussed below, which develop statistical representations of the distribution of indoor concentrations as a function of geographical, structural, and other variables desired.

Furthermore, an intermediate approach to modeling, as one alternative to a fully deterministic modeling approach just described, is to develop a stochastic model of indoor air quality, where the driving forces are only statistically known. Another alternative - with obvious practical applications - is to leave out the house model altogether, and concentrate on the potential of the site to provide a high rate of radon entry. An example of the first approach (though not applied to radon as yet) is provided by Haghighat et al. (1988). An example of the second approach is provided by the attempts to provide measures or simple indices for characterizing sites with respect to their radon source potential. The purpose of such characterization is not to predict indoor radon concentrations and exposures as such, but to predict locations where 
control measures or modified construction practices would be desirable. Since it now seems clear that high radon entry rates into houses can occur also from soils having ordinary radium concentrations but with high relative permeability (Nazaroff and Sextro 1989), these indices have attempted to combine these two governing parameters (emanating fraction of radium and relative permeability) to obtain a single and simple index for radon source potential of homogeneous soils (DSMA 1983, Nazaroff and Sextro 1989, Kunz 1988, Kunz et al. 1989, and Yokel 1989). While the index is a useful measure to characterize sites where the soil is relatively homogeneous, sites with considerable inhomogeneities abound in the Northeast. At these sites, channeling of soil gas along strata or shear zones of high relative permeability passing near the house sub-structure appear to provide pathways for high rates of soil-gas entry into some houses, while their neighboring houses have much lower entry rates.

A third alternative is to build a model based on physical understanding of the major phenomena, but attempt to determine the values of the unknown parameters by short term "diagnostic" tests and procedures. This approach attempts to model indoor radon concentrations by focusing on building up a manageable simulation structure by making judicious simplifying assumptions. A recent attempt (Revzan 1989) focused on two New Jersey houses would serve as a good example of the state of the art in this direction. The model incorporates distinct models of soil-gas transport, its entry and removal from the basement, and its entry and removal from the upstairs living space. The occupant behavior, geological and structural factors are not modeled, but derived from fitting the observed data to observed concentrations over a limited period under constraints of the sub-models. The derived parameters and the sub-models are then used to predict radon concentrations for other periods. Similar short-term diagnostic methods to predict long-term performance have been recently developed successfully for residential energy use by Subbarao and Balcomb (1988). There is hope that a similar approach can be developed and validated for indoor radon concentrations. Another diagnostic effort, aimed at characterizing the sub-slab structure (in terms of communication and permeability) for predicting effectiveness of sub-slab ventilation technique in reducing indoor radon concentrations, is described by Matthews et al. (1989).

\section{Predictive Models for the Housing Stock}

As discussed in the previous section, predictive models for housinz stocks are based primarily on a physical model, combined with data on relevant parameters from statistical samples of the housing population. Figure 16 illustrates the major components of such a model, which would yield the indoor concentration for the entire housing stock, but - more importantly - as a function of house type, geographic area, etc. It could also be used to assess the effectiveness of proposed control strategies. The "kernel" of such a model is some form of the physical models discussed above.

Assuming the existence of a physical model, the key problem in predictive modeling for the housing stock is the development of a data structure representing the key characteristics of the housing stock. Although some of the relevant information is available in the form desired, this is the exception, rather than the rule. And there has certainly not been any survey to acquire all the required information in a single statistical sampling of the housing stock. This information would include key structural and operational characteristics, relevant geological parameters, and associated meteorological data, to mention the major classes of data required. At present, it is necessary - for such modeling applications - to piece together partial information from different sources, in the absence of a consistent or complete set of the information required.

To some extent, these deficiencies can be alleviated by intercomparison of derived and secondary data sources obtained from a number of related surveys that contribute 


\section{ELEMENTS OF A GENERAL \\ INDOOR POLLUTANT EXPOSURE MODEL}

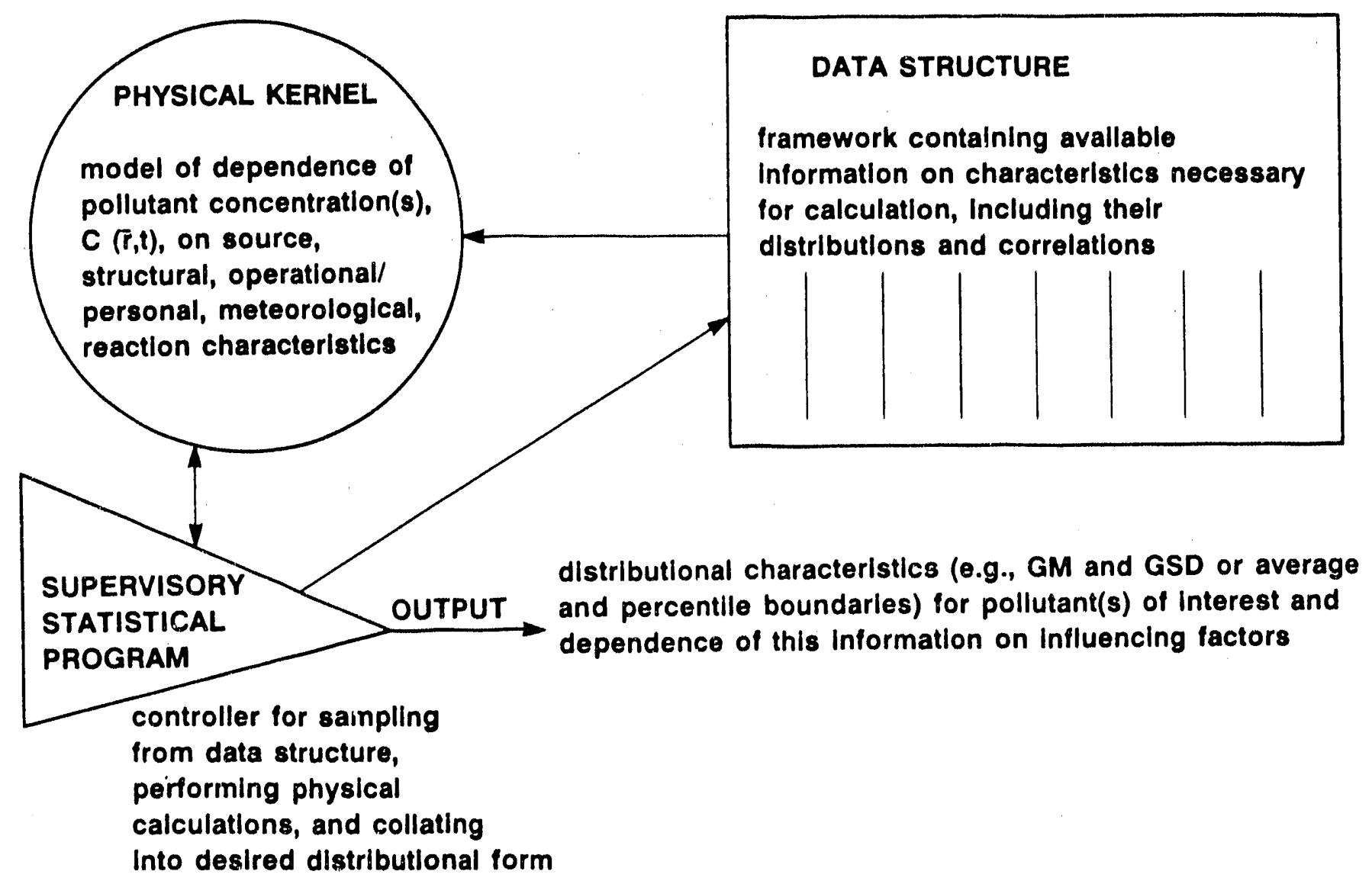

XPL $331-8003$

Figure 16. The basic elements of a predictive model for the housing stock.

These elements include a physical kernel (derived from models such as those indicated in Figs. 13 and 14), a data structure for characterizing factors affecting the indoor level, and a supervisory and statistical module for organizing calculations and results into the form required. 
to the information that the model attempts to represent. In fact, such intercomparisons can lead to identification of data-gaps, survey errors, and inadequacies in the present understanding of the physical phenomena involved. Examples of such surveys would be those providing data on distribution of air-exchange rates, housing construction types, soil permeabilities and radium concentrations, personal exposure measurements, concentration surveys, experimental and demographic data on lung cancer rates, and so on.

For example, from an examination of the survey data on distribution of indoor racion concentrations, soil relative permeabilities, and radium concentrations discussed below (Section 3 ), it appears that our present understanding of the radon entry and removal mechanisms is inadequate to explain in detail the narrowness of the radon concentration distribution relative to the breadth of distributions of soil radium concentrations and soil relative permeabilities, as discussed in the next section. A preliminary analysis of BPA data from 84 houses (including soil properties, indoor radon concentrations, and air-exchange rates) leads to similar conclusions.

A major example of this general approach to modeling is the work of Traynor et al. (1989), who have constructed a model for assessing residential concentrations of combustion-generated pollutants, in particular $\mathrm{CO}, \mathrm{NO}_{2}$, and respirable suspended particulates. There is some hope that development of predictive models for the case of radon and other pollutants will be eased by the acquisition of data from the (roughly) 5000-home national radon survey being conducted by the EPA. This would provide a basic data set on house characteristics, including even appliances, that will be the first self-consistent set of information serving as a basis for predictive modeling. Furthermore, the indoor radon concentrations will automatically be available for testing and validating the model. It is important to realize that the results on house characteristics will also serve as a primary resource for predictive modeling for other indoor pollutants. (Unfortunately, however, the associated questionnaire was not designed to include a few key questions that would be very helpful for these other pollutants.)

Finally, predictive models of exposures in the housing stock can be built up not only based on the physical survey results, but incorporating the ongoing process of increased public awareness, homeowner testing, development of public policy, implementation and effectiveness of remedial measures, and resulting changes in population risks and statistical deaths (Small and Peters 1988). Though such attempts are relatively new, they represent a potentially fruitful line of investigation in attempting to analyze the impact of various public policy options on the population exposure and risk.

\section{Data for geographic radon modeling}

Radon arises primarily from radium present in the ground, as a result of which numerous attempts have been made to correlate indoor concentrations with the radium content of soil and other relevant parameters. One of the major lines of work has been the use of a "radon index number," such as discussed above, as an indicator of the indoor radon potential. Such indices typically involve both the radium concentration and the permeability, since the latter is one index of the transport of soil gas indoors.

In addition, there have been a number of studies attempting simply to examine the correlation between radium concentration and indoor radon levels, an example being that of Kothari and Han (1984). With the upsurge of interest in means to identify high-radon areas or sites, these efforts have increased, of ten with application in known high-radon areas (cf., for example, Reimer and Gunderson 1989 and Duval et al. 1989).

One natural resource for such efforts, and one that could serve admirably in the context of a geographic predictive model, is the data from the National Uranium Resource Evaluation 
(NURE), as part of which the National Aerial Radiometric Reconnaissance (NARR) measured the gamma radiation from the top $0.5 \mathrm{~m}$ of soil or rock of the United States as an indicator of uranium concentration.

In an early examination of the utility of that data for purposes of assessing indoor radon on a geographic basis, Moed et al. (1985) examined the data for California and the Pacific Northwest. Their report provides a basic description of the NARR data, as well as information on concentrations in the areas mentioned and results from ground-truth testing of the data in selected areas of the Pacific Northwest. More recently, the data have been reformulated into a more generally useful data base (Revzan et al. 1988), some results of which are worthy of description.

\section{The NARR Database of Surficial Radium Content}

The NARR survey was carried out by seven subcontractors, each of which was responsible for a number of National Topographic Map Series quadrangles of $2^{\circ}$ longitude by $1^{\circ}$ latitude or $140-200$ by $112 \mathrm{~km}$. Data were collected along primary flightlines, separated by intervals of 5 $\mathrm{km}$ or less in regions of interest and $10 \mathrm{~km}$ in others, and along tie lines, running perpendicular to the primary lines and separated by intervals of 20-30 km. Counting rates for gamma rays of interest, among them that of ${ }^{214} \mathrm{Bi}$, a decay product of radon, were recorded at intervals of 1 second, so that each datum represents the radiation from an area whose diameter is on the order of $50-100 \mathrm{~m}$. The flightline itself is covered completely, with considerable overlap between adjacent points, but there is obviously considerable missing information for the territory between flightlines. The spectrometers were calibrated using concrete pads containing known concentrations of the nuclides of interest. The data given as ppm equivalent uranium, which we express as $\mathrm{Bq} \mathrm{kg}^{-1}$ or $\mathrm{pCi}^{-1}$ radium, are actually better indicators of porespace radon than they are of uranium or radium in soil granules.

Although the complete NARR data are available on magnetic tapes, the variety of formats used and the large storage requirements make use of the data inconvenieni at best. To create a database which could be retained on a single storage device, the east-west flightlines were divided into 125 intervals, each covering $0.016^{\circ}$ of longitude, and the north-south flightlines into 80 intervals, each covering $0.025^{\circ}$ of latitude; each interval represents roughly $1.6 \mathrm{~km}(1$ $\mathrm{mi})$. Although the complete data can only support a resolution of $10 \mathrm{~km}$, since that is the usual flightline spacing, the higher resolution of the database may prove useful if a technique for using the tie lines as a basis for interpolation is developed.

The reduced data are stored by quadrangle in ASCII files. For each interval, the stored data include: the central longitude and latitude, the flightline number and direction, the Federal Information Processing System code for the state and county in which the point lies, and the arithmetic mean and standard deviation of $\mathrm{Ra}\left(\mathrm{pCi}^{-1}\right), \mathrm{Th}(\mathrm{ppm}), \mathrm{K}$ (percent), atmospheric ${ }^{214} \mathrm{Bi}$ (equivalent $\mathrm{pCi}^{-1} \mathrm{Ra}$ ) and gross gamma (counts). The subcontractor's code for the rock or soil type is also stored; codes used by different subcontractors are not identical, so that the usefulness of the geological information is limited. For each quadrangle, a separate file contains information on the subcontractor, calibration of detectors, and aircraft type, averages of the nuclide concentrations, temperature, and atmospheric pressure for each flightline, and a list of the states and counties covered by the main data file, with the number of data points lying in each. The entire database requires approximately 350 megabytes of storage.

\section{A U.S. Radium Map}

Figure 17 is a map of the contiguous 48 states on which each dot represents the average of the stored $(1.6 \mathrm{~km})$ radium concentrations for the surrounding $20 \times 20 \mathrm{~km}$ area. Data from primary and tie lines are treated identically. The radius of the dot indicates the magnitude of 


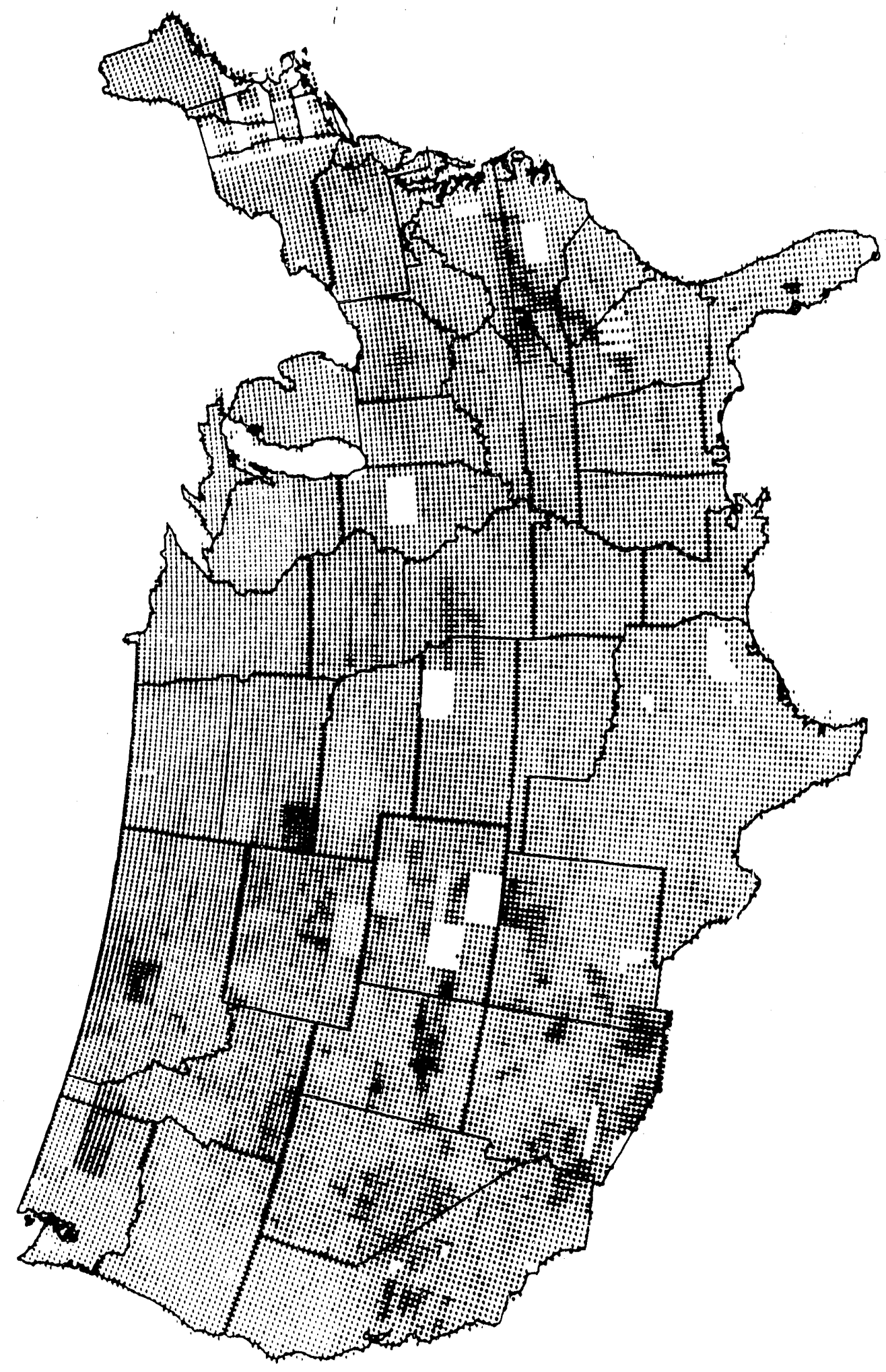

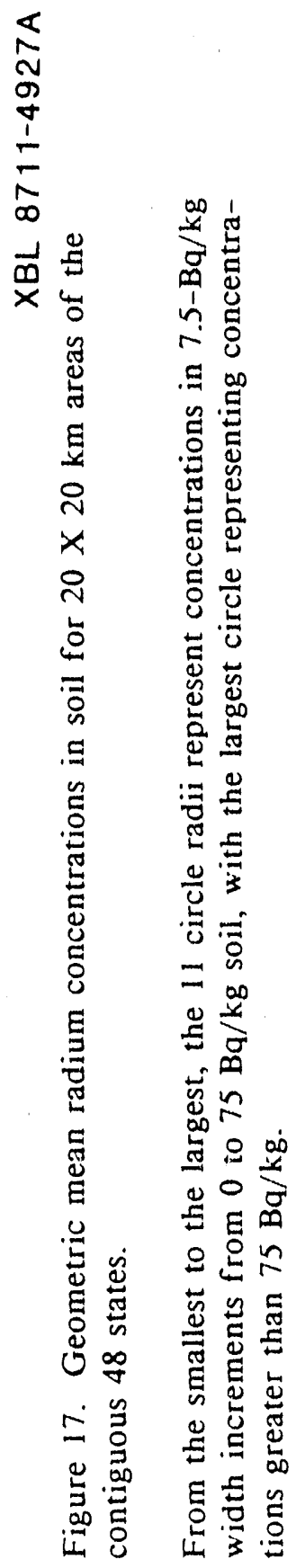


the concentration (see caption). Blank areas occur where data were not available. Areas of the map with higher radium concentration appear darker than those of low concentration.

The map reveals that certain areas appear sufficiently different in character from their surroundings to lead to suspicion that there might be problems involved in attempting to compare data from subcontractors using different detection systems, despite their use of the same calibration pads. The problem is perhaps most clearly exhibited in two quadrangles of central south Wyoming and central north Colorado which appear lighter in shading than adjacent quads. In both, all of the $20 \times 20 \mathrm{~km}$ averages are in the lowest range; the surroundings are at least one range higher. The means of $R a$ for the two anomalous quadrangles are 6.3 and $8.1 \mathrm{~Bq} \mathrm{~kg}^{-1}$, while the mean $\mathrm{Ra}$ of the six surrounding quadrangles in $33.3 \mathrm{~Bq} \mathrm{~kg}{ }^{-1}$; so that the problem appears to be with the uranium calibration alone. However, the subcontractor used a detection system with identical calibration factors, presumably the same system, in a number of other quadrangles, none of which stands out from its surruundings. The cause of the Wyoming-Colorado anomaly remains unclear.

Other poszible calibration anomalies appear in southwestern South Dakota (a single quad surveyed by a different subcontractor from the surrounding quads), eastern Washington and western Idaho (two quads, each flown by a different subcontractor), and San Jose, California, which will be discussed below. The average radium concentration of the territories surveyed by individual subcontractors ranges from 20 to $32 \mathrm{~Bq} \mathrm{~kg}^{-1}$, but the variation may be explained, at least in part, by the different areas of the country flown by each; the question of the importance of subcontractor performance remains open. The problem of smoothing out artificial discontinuities in a $\mathrm{Ra}$ map is currently under investigation (e.g., Duval 1989).

The following areas, which the map reveals as having noticeably high radium concentrations compared to their surroundings, have also been found to have high radon concentrations in homes (Nero et al. 1986): eastern Pennsylvania-northern New Jersey (Reading Prong), eastern Tennessee-western North Carolina, eastern Washington-western Idaho (Spokane-Cour d'Alene), and western Florida (phosphate-bearing land). Apart from Florida, where the situation is well understood, and Tennessee, the mean levels of radium in these areas are not extremely high, and, in any case, the nature of the relationship between the means of radium in soil and radon in houses remains to be established.

For the sake of completeness, it is worth mentioning the regions which are known to have high radon concentrations but do not appear, according to this data reduction, to have high radium levels and those which, conversely, have high radium concentrations but in which high radon levels have not, at least as yet, been found. In the former category there is just one region: the Red River valley of North Dakota. While the map shows the valley to be somewhat higher in radium than the territory to the east and west, the level is the same as that of the territory to the south, so that it is difficult to infer from radium concentrations alone that this might be a region of interest. In the latter category, there are a number of obvious examples: central California, southern Arizona-New Mexico, a number of regions in the Rocky Mountain states, central New Hampshire-southern Maine, and most conspicuously, southwestern South Dakota. Some of these are known to be areas in which uranium deposits occur; some, as suggested, may stand out because of calibration problems; some may be worthy of specific monitoring efforts.

\section{Mapping of Quadrangles}

The quadrangle is the most natural mapping scale between the U.S. as a whole and the very detailed studies which will be necessary for regions of particular interest. Maps of soil radium for individual quadrangles can be useful in analyzing discontinuities which appear to the U.S. map, in pointing out discontinuities not apparent on the larger scale map, and in identifying smaller regions of high concentration. Since there are fewer than 500 quadrangles, the number 
of maps is large but not unmanageable.

A quadrangle of particular interest is San Jose, located entirely in California between longitudes $120^{\circ}$ and $122^{\circ} \mathrm{W}$ and between latitudes $37^{\circ}$ and $38^{\circ} \mathrm{N}$, which appears on the U.S. map as a region having radium concentrations generally in the third or fourth range, i.e., between 40 and $80 \mathrm{~Bq} \mathrm{~kg}^{-1}$. The elevated radon concentrations which are suggested by these figures have not been reported, although it is not clear that sufficient measurements have been made to have discovered them. A radium map of the primary flightline data for this quadrangle at the scale of $10 \times 10 \mathrm{~km}$, using the same technique used in the creation of the U.S. map, is provided as Figure 18, whose caption provides details. The map reveals three regions of elevated concentrations with the possibility of a fourth beginning at the right bottom. The regions of high concentrations are surrounded by areas of much lower levels. A similar map of the tie line data differs markedly in character.

The occurrence of temperature inversions in the area suggests that examining the relationship between the corrected radium level and the temperature during data collection might be productive. As it turns out, the San Jose quadrangle exhibits a fairly high positive statistical correlation between the average temperature for the period of data collection for a primary (east-west) flightline and the average radium concentration ieported for that flightline (Pearson correlation 0.78 ; probability of the absence of correlation 0.000 ). The positive correlation does not appear for the tie lines, nor does it appear for the other nuclides for either the primary or tie lines; neither does it appear for any of the surrounding quadrangles. Subsequent ground truth studies (Wollenberg and Revzan 1989) indicate that the high radium concentrations suggested by the NARR data for the San Jose area are misleading.

The appearance of quadrangles whose radium concentrations are discontinuous with the surrounding quadrangles (cf. Fig. 17), or are in some other way anomalous, suggests that corrective measures be taken wher the data are actually used. The simplest of these measures, which might be used where a calibration problem is suspected, is to apply a correction factor so that the transition to the surrounding quadrangles is smooth (Duval 1989). A somewhat more sophisticated approach is to use the data from the tie lines, weighted to adjust for the smaller number of points if desired, together with the primary flightline data, to create a new set of values for the quadrangle; mathematical techniques to carry out this procedure, which involve interpolation and smoothing, are readily available. Finally, it is possible to create contour maps from the individual data points. Such maps can be compared with similar maps for adjoining quadrangles, and the data for one or more of the quadrangles can be adjusted to produce a smooth transition.

The Statistics of Radium in the Soil, Indoor Radon, and Other Factors

On the basis of the 22,172 averages used for the $20 \times 20 \mathrm{~km}$ map, the geometric mean (GM) radium concentration for the U.S. mapping is $25 \mathrm{~Bq} \mathrm{~kg}^{-1}$, the geometric standard deviation (GSD) is 1.6, the arithmetic mean (AM) is $27 \mathrm{~Bq} \mathrm{~kg}^{-1}$, and the arithmetic standard deviation (ASD) is $12 \mathrm{~Bq} \mathrm{~kg}^{-1}$. These parameters are useful for making comparisons among regions, but they are misleading in that each datum is treated as if it were itself an observation rather than as an average of data which are themselves averages of original observations. Since the data base retains the number of observations and the AM and ASD for each $1.6 \mathrm{~km}$ flightline datum, one can calculate an ASD for each $20 \times 2.0 \mathrm{~km}$ region and then an ASD for the contiguous U.S. On the basis of the original $24,591,855$ measurements, this number is $21 \mathrm{~Bq}$ $\mathrm{kg}^{-1}$. Calculation of the GM and the GSD on the same basis involves approximations, since the GM and GSD of the observations have not been retained in the reduced database. If data are lognormally distributed, the $A M$ and ASD may be used to calculate a GM and GSD, yielding $21 \mathrm{~Bq} \mathrm{~kg} \mathrm{~g}^{-1}$ and 2.0 , respectively. As a check on this result, a GM and GSD may be calculated for each $1.6 \mathrm{~km}$ datum, after which overall parameters may be calculated from these 
3

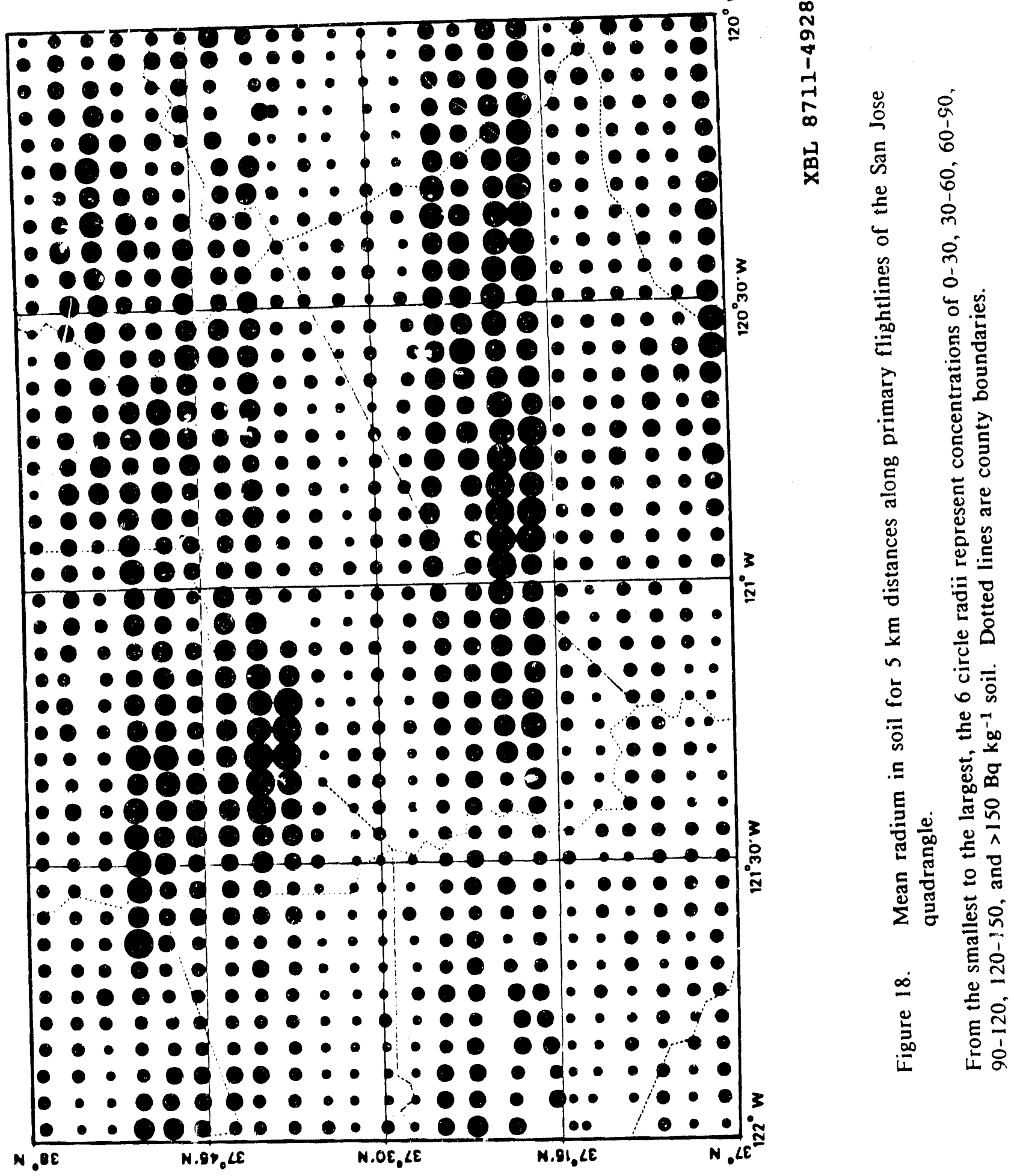


numbers: the GM on this basis is $22 \mathrm{~Bq} \mathrm{~kg}^{-1}$ and the GSD is : gain 2.0.

If we now consider that each original observation itself represents an integration of several huncired square feet and that large areas of the country remain unobserved due to the relatively large distances between flightlines, it is apparent that the actual GSD of observations of radium in soil made at ground level, each representing an area the size of a house, s likely to be somewhat greater than 2.0. The GSD of measurements of radon concentration in houses in the U.S. is approximately 2.8 (Nero et al. 1986). Suppose that the indoot adon concentration is the product of the soil gas radon concentration, represented by the radium concentration, and some other lognormally distributed factor or combination of factors. Given the fact that the logarithms of GSDs add in quadrature, two factors contributing equally to the variation in radon would each have a GSD of 2.1 , which is very close to the calculated GSD for radium. The observed variation in radium in soil may thus account for roughly half the observed variation in indoor radon.

A more comprehensive view of the factors leading to the observed distribution of radon concentrations must take account not only of the radium concentration, but also of variability in permeability and substructure characteristics, as well as differences in ventilation rate associated with varying characteristics of the building shell. In fact, it has been observed (Grimsrud 1988) that the "leakage area" of structures (see Appendix B for discussion) also has a distribution with a GSD not far from 2.0. Given the uncertainties involved, it is useful to observe that, if the grand distribution of radon concentrations has a GSD of about 3.0, this would be accounted for by three underlying factors, each varying independently of the other with individual GSDs of 1.9. This is similar to the GSDs just mentioned for the radium concentration and the leakage area, but has the difficulty that it is much smaller than the GSD associated with soil permeabilities.

As discussed in Nazaroff et al. (1988) and elsewhere, soil permeabilities vary over a very wide range, in fact by a factor of about a million from coarse sand or gravel to fine clays. Given that a noticeable fraction of underlying natural soils is associated with these extreme values, it appears that the overall distribution of soil permeabilities must have a GSD of about 10 or 15 , very much larger than the other variances involvet in the issue of indoor radon. Thus if the full variability of soil permeability were expressed in a linear way in the observed concentrations, then the concentration distribution would also have a GSD of 10 or more, which is clearly not the case.

This leads to the conclusion that the indoor concentration is not linearly dependent on soil permeabilities, a conclusion that is of course suggested by other evidence. That is, at very low permeabilities, diffusion of radon (rather than soil-gas movement) must be the dominant transport mechanism, and at very high permeabilities, other factors (including the integrity of the understructure itself) must limit the extent to which extreme values of permeability are expressed as high indoor concentrations. The precise manner in which the full range of permeabilities is "compressed," in term of its expression in radon concentrations, is not understood, but that this occurs is clear. One of the main objectives of the physical modeling of radon entry discussed above is to understand the interaction of these factors in yielding the indoor concentration, as a basis for understanding the occurrence of high concentrations and how remedial measures can reduce them. It is in the context of predictive models that physical models can be utilized for understanding the association of high concentrations with different geographic areas or housing characteristics.

Unfortunately, although the radium data are available in a form suitable for such modeling and assessment purpose, the same is not true for nther information In narticular, informatinn on other key parameters, such as soil permeability and house characteristics - while available to some degree - is not formulated into consistent data bases that can be used easily for evaluation purposes. Thus, for example, Soil Conservation Service maps provide some 
indicators of water permeability, which in turn may be related to permeability to gas flow, but this information has not become available in a form that is easily used, other than potentially for assessing the radon potential of localities. Similarly, some degree of information is available on housing characteristics, but not from a consistent survey that provides, of itself, a dependable basis for assessing indoo: concentrations. As noted above, the EPA's national radon survey may alleviate this situation significantly.

For the present, improvements in our capabilities for representing transport processes are the key to understanding the occurrence of high indoor radon concentrations, the effectiveness of control techniques, and the potential for formulating models that can be used, not only to identify areas and structure types with a high radon potential, but to assess the effectiveness of control strategies aimed at reducing exposures.

\section{Control Techniques}

In principle, indoor concentrations of radon and its decay products are influenced by entry rates, ventilation rates, and other removal or transformation mechanisms, the last category affecting primarily the decay products. It has become clear that the main influence on indoor concentrations - and hence the main contributor to the overall variance - is the entry rate, with a somewhat smaller dependence on ventilation rates. As for the influence of decayproduct interactions, although there is clearly some variability in the equilibrium factor, it is typically in the vicinity of 0.4 , with almost all indoor environments within a factor of two of this.

Just as variability in the entry rate is the major contributor to the observed variance in indoor concentrations, so techniques that limit the entry rate are found to have the greatest potential for effecting large reductions in indoor concentrations, although techniques that provide greater ventilation (or even that remove radon or its decay products from the indoor air by cleaning methods) can be useful in some circumstances. We here review briefly some of the evidence and current research on methods to reduce entry of radon into the indoor atmosphere. More thorough discussions appear in such works as Nazaroff and Nero 1988 and NCRP 1989.

\section{Techniques that reduce entry of radon from the soil.}

Consistent with the observation that most racion enters single-family houses via the flow of soil gas from the ground through the substructure, radon entry can be reduced by sealing entry routes or by altering the pressure differential between indoors and the soil that drives the transport of soil gas. Such methods are reviewed in Scott $(1988 ;$, but we here mention some of the more important work reported on such techniques.

An initial and important bodv of work was presented in a series of three conferences sponsored by the Atomic Energy Control Board in 1978-80 (AECB 1978-80), where a number of efforts employing methods for sealing or altering the pressure field were described. Similarly, efforts at Grand Junction and elsewhere, where elevated indoor levels associated with the indiscriminate use of uranium mill tailings had been disccvered, prompted work on control techniques, one example being the work of Culot and collaborators on sealants (Culot et al. 1978).

With the discovery of high radon concentration in the general building stock beginning in the late 1970s, efforts to reduce concentrations in such structures began in earnest by the early i9ôs, atihuoush - civen to this day - such efforts are not reported very effectively in the literature. Notable exceptions are studies of control techniques in selected small groups of houses. Turk et al. $(1989 a, b)$ report on control techniques in a group of homes in the 
Spokane River Valley, where - beginning in 1985 - they examined the effectiveness of techniques that either pressurize or depressurize the layer of soil immediately beneath the basement slab, as well as the use of basement overpressurization, air-to-air heat exchangers, crawlspace ventilation, and sealant techniques (particularly in conjunction with subslab ventilation). In general, it was subslab ventilation (especially used in a pressurization mode) that effected the most substantial reductions in indoor concentrations. This work has included follow-up studies of previously installed remedial systems (Prill et al. 1989).

A series of meetings sponsored by the EPA since 1986 have included a number of reports on control techniques, although these are sometimes almost anecdotal. An exception is a report of Henschel and Scott (1986) on remedial measures in a group of homes in Pennsylvania, where a variety of soil-ventilation techniques were employed in homes with concrete block-wall basements. Other papers at the same meeting (APCA 1986) and others (APCA 1987 and USEPA 1989b), as well as the international meetings listed in Table 1 , have included substantial work on control techniques. Papers, for example, by Erickson (1987) and Cliff et al. (1987) are indicative of the efforts in European countries. Finally, the effectiveness of the methods indicated above for even extreme indoor levels is demonstrated by the reduction of high levels in Clinton, New Jersey (Osborne 1987).

A basic question is the longevity of control systems. Experience with the systems installed in Spokane indicates the need for some degree of care in avoiding degradation of performance or defeat of the systems (Prill et al. 1989), observations that also come out of the work of Nitschke et al. (1988).

Finally, an area recently receiving more attention, on the part of both governmental agencies and those who develop building codes, is new housing. Ultimately, it is expected that, in areas where radon concentrations tend to be significantly higher than average, new buildings ought to be constructed so that controls are easily implemented where necessary. The DOE, the EPA, several States, and professional organizations have activities in this area. Several reports of new-home construction techniques appeared recently in USEPA $1989 \mathrm{~b}$.

\section{Detailed studies of radon entry and the influence of control techniques}

In some cases, groups of homes appearing to require remedial measures have been used to investigate in detail the mechanisms for radon entry and the influence on these processes of different types of control techniques. A principal example of this approach is a group of 14 homes selected in New Jersey, of which 7 served as an initial group on which instrumentation for continuous real-time monitoring of radon concentrations and related environmental variables was installed (Sextro et al. 1987). Shortly thereafter, an additional group of 7 homes was instrumented, in which a somewhat different regime of experimentation was instituted (see, for example, Hubbard et al. 1988). These efforts had several related objectives: (1) to investigate the dynamics of the entry process (cf. Revzan 1989 for such an analysis), (2) to study the effectiveness of remedial techniques and to observe, in the process, their influence on entry mechanisms, and (3) to examine the effectiveness of diagnostic measurements and procedures in characterizing entry and in choosing associated remedial techniques (cf Turk et al. 1987). Analysis of the results from the studies in this group of homes continues, with the homes also serving as a test bed for continuing projects (e.g., Sextro et al. 1989 and Hubbard et al. 1989).

Some studies have also been undertaken to examine transport processes, and related control techniques, in controlled situations. A principal example is an experimental study currently under way, where small basement-like structures are being emplaced in well-characterized and reiativeiy uniform sites, with an array of instrumentation in the soii and structure to examine the influence of environmental conditions on radon generation, transport, and entry over year- 
long periods of time (Fisk et al. 1989). In parallel a substantial program developing improved simulation models is underway, in part to represent adequately the dependence of the observed variations with time, moisture conditions, and soil parameters.

\section{Other types of control techniques}

In some cases, the major contributor to high indoor levels is not a high rate of entry from the soil or, even when it is, it is deemed necessary to reduce indoor levels by means other than reducing entry from the soil. One class of such cases is that where radon in water is a major contributor to the indoor concentration. The basic approaches to reducing entry via water supplies in such cases are to hold the water long enough to permit substantial decay of radon (which has a four-day half life), to aerate the water to effect transfer to air outside the indoor atmosphere, or to filter the water, e.g., by using a charcoal trap. Papers on these topics appear in USEPA 1989b. A significant difficulty with the use of charcoal is the accumulation of longlived radon decay products, which then pose a radiation hazard because of their gamma activity. Although, as discussed above, it appears that domestic water supplies account for only about $5 \%$ of observed indoor concentrations on the average, a sufficient number of homes have elevated levels (at least by the EPA standard) due to entry via water supplies, that significant attention must be given to means for controlling such entry.

A more generally significant question is the potential for using increased ventilation as a means for controlling indoor levels. Given that in only a small portion of cases are excessively low ventilation rates the major cause of elevated indoor concentrations, this approach is ordinarily not the most effective one. On the other hand, increased ventilation can provide for modest decreases in indoor concentrations on a long-term basis, can be used (in extreme cases) as an interim measure for reducing levels substantially (i.e., until a permanent solution is implemented) and, in structures where unusual building methods are employed to reduce infiltration far below the average, mechanical ventilation is typically advisable, not only for avoiding high radon concentrations but for preventing excessive levels of a variety of other indoor pollutants.

The role of general ventilation techniques in the control of indoor pollutants is reviewed in Fisk et al. (1984). The effectiveness of mechanical ventilation using air-to-air heat exchange to recover energy that would otherwise be lost for control of radon concentrations was studied by Nazaroff et al. (1981), and the overall performance of such systems with respect to energy was evaluated by Fisk and Turiel (1983). More recently, the general effect of mechanical ventilation on indoor radon levels, including potential increases in concentrations associated with exhaust ventilation, were evaluated theoretically (Mowris and Fisk 1988).

Finally, the remaining approach tor reducing indoor concentrations is to employ methods that remove radon or its decay products from indoor air itself. Generally, the removal of radon gas, by for example charcoal cleaning, has not been deemed to be as effective as reduction of radon entry, although some effort has been devoted to evaluating this method (Bocanegra and Hopke 1987).

Much more attention has been given to the use of filtration or electrostatic precipitation on the removal of decay products, since it has been established that marked reduction in the EEDC can be effected using such techniques. Such work is reviewed briefly in Section $V$ and more thoroughly elsewhere (e.g., in Jonassen and McLaughlin 1988). As discussed there, because of the particulars of decay-product behavior, including attachment to airborne aerosols and the differing deposition rates of attached versus unattached decay products, the large reductions that are seen in EEDC are not thought to be reflected in correspondingly large reductions in the dose to the lung. In fact, according to the dosimetric calculation of James (1988), the radon concentration itself may be a better indicator of dose than the EEDC. In any case, 
because of the ambiguities in effect of air-cleaning methods, this is not the approach of choice in reducing doses in homes having high radon concentrations. 


\section{TECHNIQUES AND PROTOCOLS FOR MONITORING INDOOR CONCENTRATIONS.}

The availability of techniques for measuring concentrations of radon and its decay products is a clear prerequisite for understanding the occurrence and behavior of these radionuclides in the indoor environment. Measurement capabilities are also necessary for purposes more specifically associated with risk assessment and control strategies. This section briefly indicates the techniques available for monitoring in houses, not for research on radon or decay-product behavior, but primarily for purposes of public policy or action.

\section{A. Measurement Techniques}

Measurement of the airborne concentration of radon or its decay products is based on detection of the radiation associated with radioactive decay. This radiation includes, not only the alpha and beta radiation indicated in Figure 2, but $\mathrm{X}$ or gamma radiation that is the immediate product of decay from the "excited" state in which it is left to its "ground" or unexcited state. Any of these forms of radiation is capable of creating ions in materials through which it passes, and measurement methods are based on detection of this ionization or its aftermath.

In spite of this uniformity in the basic principle of detection, practical measurement techniques take widely different forms, substantially affecting their range of application. Specif ic techniques are designed for measuring the concentration of radon, the EEDC, or indeed - the concentration of individual decay products. Some techniques are suitable for short-term measurements, where a "grab" sample of air is taken, or for continuous real-time measurements, where repeated samples are taken. Other techniques are designed for intermediate to long-term sampling, where only the average concentration is sought. Some techniques may be self-contained, requiring the presence of a moderate to large piece of equipment at the site of measurement, while other techniques deploy a small sampler or detector, which must be returned to a central analytical laboratory for readout. Finally, some techniques are inexpensive or easy to use, while others are suitable for use only in the context of experiments carried out by experienced scientists.

Measurement techniques are treated in a number of specific instrumentation reviews (Budnitz 1974, George 1980, Nazaroff 1988a, NCRP 1988). We here mention only the techniques suitable for wide-scale use and the efforts to calibrate and test them.

\section{Methods}

Widely-used techniques that are suitable for use in monitoring large numbers of houses include the following:

Grab-sample monitoring for either ${ }^{222} \mathrm{Rn}$ or its decay products can be performed using highly portable self-contained instruments that use a scintillation material to measure alpha particles emitted in a small collection cell (for ${ }^{222} \mathrm{Rn}$ ) or from a filter (for the decay products). The entire measurement is completed within some tens of minutes, therefore only giving the concentration at a specific time. Considering the potential variability of concentration with time (cf. Section III.A), this does not give a direct measure of the average exposure rate in a home.

A charcoa! sampler with dimensions on the order of $10 \mathrm{~cm}$ can be used to collect ${ }^{222} \mathrm{Rn}$ 
over a period of a day to a week, then sealed and returned to a laboratory, where the gamma radiation from decay products contained in the sampler can be measured (Cohen and Cohen 1983, Cohen and Nason 1986). This technique is not suitable for more than one week because late in this period much of the ${ }^{222} \mathrm{Rn}$ collected at the beginning will be gone because of its 4-day half life.

An etched-track detecter with dimensions of only about $3-4 \mathrm{~cm}$ (or, in some versions, more) can be placed in a home for periods from weeks to a year, then returned to a laboratory where the plastic detector material is etched to show tracks left by the passage of alpha particles from the decay of ${ }^{222} \mathrm{Rn}$ and it decay products (Alter and Fleisher 1981). As ordinarily deployed, such detectors have a filter permitting entry of ${ }^{222} \mathrm{Rn}$, but not its decay products (for which it is difficult to calibrate the device). This technique has the advantage of giving a long-term average concentration, which - however - might be considered a disadvantage if a quick result is desired. Beginning in 1988, etched-track detectors with larger areas (depending on rapid scanning techniques, rather than optical counting) began to be made available for short-term measurements, at least in Europe.

Continuous monitoring, based on either of the principles mentioned for grab sampling, is useful primarily for investigations of the time--dependent behavior of radon or its decay products, either in laboratory or field situations. It may also be used, however, as a convenient alternative to grab.sampling to ascertain the effectiveness of control techniques as they are being installed in a house. Moreover, simple and relatively inexpensive devices are now becoming available for the situation - probably infrequent - where occupants wish to have an active warning when radon concentrations rise above a specified level.

Several developments have occurred in large part since the reviews cited above. These include two integrating monitoring methods and a new approach for estimating past concentrations.

One of the monitoring methods recently commercialized in the United States is an "electret"based passive integrating detector (Kotrappa et al. 1988). The electret is a piece of Teflon that has induced on it a semi-permanent electrical charge, which - because of the associated field - can collect ions produced by decay of radon and its decay products. This causes a reduction in the electret's surface voltage that can be measured as an indicator of cumulative radon exposure.

The other monitoring method - mentioned above - is use of etched-track material where, instead of counting tracks optically, "spark" counting is used. This technique, employed in Europe (cf. Tommasino 1980 and Rannou 1987), can employ larger areas of material, and hence permit higher sensitivity at lower cost. However, as noted in Tymen et al. (1988), the use of larger, open detectors (i.e., without a filter or membrane that permits only radon gas to enter) can introduce significant uncertainties due to their sensitivity to the decay products.

Finally, integrating monitoring methods are utilized in epidemiological studies (such as NJSDH 1989) where past exposures of subjects are examined on the basis of current average concentrations in their homes. Recent studies, however, suggest an alternative method of such estimation, i.e., by measuring the deposited activity on glass surfaces of ${ }^{210} \mathrm{Po}$, the longlived radionuclide that results from decay of ${ }^{222} \mathrm{R} n$ and its short-lived decay products. Because ${ }^{210}$ Po has a half-life of 22 years, it may serve as a retrospective or prospective exposure measure (Samuelsson 1988). 


\section{Calibration and testing}

Significant efforts have been devoted to calibrating detectors and to testing their practical reliability, as well as the dependence of results on monitoring protocol. The testing of the detectors themselves - and, by implication, their analysis - is ordinarily carried out in exposure chambers under stable, reasonably well-characterized conditions. This approach, while a necessary component of calibration efforts, has substantial limitations: It cannot reflect the full range of conditions in which detectors are actually used. Nor can it reveal variations in detector fabrication or analysis - both factors that can affect sensitivity without extraordinarily intensive efforts. We here indicate, in general, the types of calibration and testing efforts that have taken place in recent years.

A mainstay of efforts to calibrate a variety of radon detectors in the United States has been the program at the DOE Environmental Measurements Laboratory (EML), which has exposure chambers and monitoring capabilities that permit the exposure of detectors to known atmospheres under controlled conditions. These facilities have therefore been used as a kind of primary reference laboratory for a variety of organizations in the United States, including both research groups and detector manufacturers. An effort of research groups began in 1980, whereby samplers were sent to EML and analyzed by the respective groups, who also generally maintained their own calibration facilities using - as does the EML facility - radon sources based on ${ }^{226} \mathrm{Ra}$ solutions supplied by the National Institute of Standards and Techrology. Initial results of this effort are reported by Fisenne et al. (1983). In addition, developers of commercial detectors have used this facility directly or indirectly, either by placing their detectors in the EML chamber (e.g., Alter and Fleischer $1981)$ or by calibrating their detectors in a chamber that is connected via an intercomparison system with this chamber (e.g., Kotrappa et al. 1988).

Since the much more widescale monitoring that began in 1985 and later, the EPA has undertaken a Radon Measurement Proficiency Program in which companies may submit detectors to the EPA for testing, generally by exposing them to known concentrations of radon in a Federal exposure chamber, after which the detectors are analyzed by the vendor and the results compared by the EPA with the known levels. The EPA periodically publishes a list of companies that have passed the test requirements. The early rounds of testing were performed using the EML chamber, but the site soon changed to the EPA's Eastern Environmental Facility. And whereas only 39 companies participated in the first round of tests (in 1986), the number exceeded 1000 in 1988. The primary criterion of this test is that the companies report concentrations within $25 \%$ of the "true" value. Until recently, the tests were not "blind," in that the companies voluntarily sent known detectors for exposure. They could therefore take pains that these particular samplers were treated as carefully as possible. (More recently, a system of blind testing has been undertaken, in which the companies do not know what detectors are being tested.) Any company may enlist in this program simply by contacting the EPA (Radon Technical Information Service 1988). Results from the program are reviewed in a recent report on uncertainties in radon measurements (USGAO 1989).

Still, both of the approaches above apply quite limited testing to the detectors provided by any particular vendor or manufacturer. A significant supplement to these efforts has been the program carried out at the DOE Technical Measurements Center, which has exposed detectors purchased (blind) from various manufacturers to various radon concentrations, issuing periodic reports (Martz et al. 1989b). This program has found anomalies in the performance of detector types that indicate the need for caution in their use. For a program employing large numbers of detectors, e.g., a systematic survey or an epidemiological study, determining the quality of the resulting data can be accomplished by a program of quality assurance and quality control, as discussed for example in NCRP (1988). 


\section{Field performance}

The three types of calibration and testing just described provide some basis for utilizing the various types of detectors that are now commercially available. However, this does not demonstrate their reliability in actual conditions of use. To some extent this is assured by testing their performance, in laboratory studies, in the range of physical conditions that will be encountered in actual homes (and other buildings). In addition, one has to test to make sure that the detectors are actually used properly, which of ten means in accord with the instructions provided. Again, in the context of major studies or epidemiologial surveys, this can be accomplished by a program of quality assurance and testing.

The most difficult problem in employing the array of detectors now available has to do with protocols and with interpretation, in large part the subject of the next section. We have already seen, in Sections II.B and III.A, the substantial dependence of concentration on location and time and, hence, the sensitivity of monitoring results to the protocol employed. There have, in addition, been increasing numbers of studies aimed specifically at comparing different techniques or protocols in parallel exposures.

A few such comparisons have been made incidentally. For example, in the New Jersey epidemiologial study discussed earlier (NJSDH 1989), annual-average results in living space differed markedly from "screening" results from basements. This is consistent with other data (e.g., Alter and Oswald 1987, Borak et al. 1989), which showed roughly factor-of-two differences between basement and living-space results. Furthermore, other results (cf. Table 4, in Section III.A) indicate the potential importance of seasonal variations in concentration. On the other hand, Steck (1989) has reported relatively good correspondence between overall results, given for example as the median concentration, between annualaverage living-space concentrations and EPA "screening" results for the states of Minnesota and Wisconsin. This good correspondence, a strong contrast with other (albeit limited) results, may occur because of structural, climatic, or occupancy patterns in these areas that differ from those in other parts of the United States; it may also arise, in part, because of the particular circumstances in which the comparisons were made.

The sensitivity of particular measurement protocols to the time dependence of indoor concentrations has also been suggested in several recent studies. Scott (1989) has illustrated how different patterns of variability arise from different source behavior, reaching the conclusion that several 15-day measurements over a year are required to approximate the annual-average concentration. Sextro and Lee (1989) illustrate the sensitivity of short-term sampling with charcoal canisters to variability in indoor concentrations, based on theoretical analysis and measurements in an environmental chamber. It is evident that more substantial work of this kind is necessary if measurement results are to be used properly as a basis for estimating the concentrations to which occupants are actually exposed.

\section{B. Interpreting and Utilizing Results}

The earlier discussion of concentrations in U.S. homes (Section II.A) illustrates the difficulties with proper use of monitoring results. A particular concern - as noted in the previous section - is interpretation of short-term monitoring in a non-representative location as an indication of the concentration to which people are exposed or of the fraction of houses where concentrations exceed relevant guidelines or recommendations. Thus, in surveys to determine the distribution of concentrations, or as an element in control strategies (cf. Section VI), care must be taken to interpret data consistently with the monitoring procedure used, paying attention to the period over which sampling is 
performed, the time of year during which the measurement is performed, and the location of sampling. Where the particular sampling technique and protocol does not yield directly the parameter desired, auxiliary measurements in a subset of houses and additional information (such as meteorological) may be required as a basis for interpretation. In some cases, the possibility for such interpretation can be substantially enhanced by a theoretical framework relating indoor concentrations to the factors affecting them.

\section{Techniques and strategies for identifying high-radon homes}

National or regional surveys together with some form of predictive modeling (cf. Section III.C.2) can be designed to identify geographic areas or types of buildings where high concentrations tend to occur. Once houses in an area or class are known to have a high probability of excessive concentrations, it is still necessary to monitor each house of concern to determine actual levels in individual houses. The objective of such monitoring is ultimately to obtain a useful estimate of the annual-average concentration in the living space. Achieving this simply stated objective is complicated by two factors: first, measurement techniques differ substantially in their suitability for determining this quantity; and secondly, the annual-average has an intrinsic variability because of its dependence on a variety of factors, including such obvious ones as the local meteorology and the pattern of use of the hoine teing examined. As a result, any measurement outcome must be utilized within an interpretive framework associated with the concentration guidelines being applied.

In the United States, at present, it is recommended by the EPA that the initial measurement be performed using a charcoal detector in the lowest level of the structure (USEPA 1986), yielding a result that is indicative, but that cannot be used as a basis for a decision (or, as seen in Section II, for indicating the concentration distribution).

Because it can be left in place for a year, the etched-track technique is most directly suited to measuring the desired quantity. However, even for this technique, the resulting value is not unambiguous. A single detector measures the concentration in one location, so it may not accurately reflect the average living-space concentration, even if placed in the most heavily occupied room. Thus, even aside from the intrinsic variability in the concentration due to meteorology and use patterns, every technique has limitations associated with protocol, as well as analytical uncertainties intrinsic to the technique itself. The resulting concentration therefore has an uncertainty that must be considered in any use to which it is put. However, uncertainties of approximately 20 or 30 pervent are not a great concern: the risk associated with radon exposures is assumed to be a smooth function with no threshold, so that the guidelines should not have sudden thresholds, nor should occupants' decisions ordinarily be affected by modest uncertainties in measurement results. (At present, however, because of the manner in which protocols and guidelines are formulated and used, a measurement result of $160 \mathrm{~Bq} / \mathrm{m}^{3}$ - i.e., $4.3 \mathrm{pCi} / 1$ - may cause a reaction much different than a value $20 \%$ lower.)

Utilization of the charcoal technique requires attention to the issues of variability over medium to long times, e.g., from week to week and from one season to another. Such variability can introduce substantial uncertainties, which, however, can be reduced significantly with knowledge of meteorological conditions at the time of measurement, coupled with a theoretical or experiential framework within which to make necessary adjustments. Nonetheless, there are significant limitations. For example, in an area with moderate summertime meteorological conditions, it is unlikely that a low reading from summer monitoring is a useful indicator of the annual-average concentration. As discussed below, results from charcoal sampling can be more useful in the proper interpretive framework. However, this framework is only now being developed. 
Finally, grab sampling - because it does not average even the very large daily variations known to occur - gives little basis for any kind of decision, not even about whether or not to monitor further, unless performed a number of times, as indicated below. Ironically, single grab samples can be used - with proper interpretation - to indicate area means (and even distributions) in a regional survey (Scott 1983b). But there is presently no adequate measurement methodology or interpretive framework for determining that, in a single house, one low reading indicates a low annual average or that a high reading indicates the need for remediation. In any area where high concentrations occur, almost every grabsample measurement would have to be followed by an integrated measurement, which is to say that the grab-sample measurement has very limited utility. An alternative is to perform a number of grab samples in a house, effectively simulating an integrated sample. (Furthermore, it is important to recognize the importance of grab sampling or real-time measurements for monitoring the concentration during attempts ro implement control measures in a specific house.)

As indicated, even if a useful measurement technique is employed, some interpretation has to be applied and uncertainties considered in utilizing the result within a system of guidelines. This extends even to the consideration of temporal variability from year to year. But the most difficult variability to consider is that arising from different patterns of occupancy and operation of homes, as well as different personal habits (such as cigarette smoking). Creating a monitoring and control strategy that copes explicitly with all such matters is probably not practical. In fact, considering that houses typically have a sequence of occupants, it may be best that the concentration estimate sought from any monitoring approach and interpretive framework be that characteristic of some "normal" use pattern and not be linked to personal habits but only to key operational questions.

Perhaps the dominant operational question is the amount of ventilation, either through open wi Jus or because of mechanical (heating, ventilating, or air-conditioning) systems. As an example of the kind of criterion that might be used, in many areas windows are normally kept closed during the heating season, and this is the period during which the higher indoor concentrations occur. If current occupants leave windows open during the heating season, the concentration estimate should probably adjust monitoring results to the "normal" condition in some way, at least for subsequent occupants. Formulating a consistent approach for handling such complexities, while sometimes perplexing, is a necessary part of a long-term radon control strategy.

\section{Framework for interpretation and control.}

Just as the results of a regional survey must be interpreted in light of design and objectives of the survey, the results of monitoring in an individual house must be used intelligently. First, using the result to estimate an annual-average concentration in the living space ordinarily requires some degree of interpretation. Secondly, the manner in which this estimate is used in making a decision about controls depends on how it fits into the applicable guidance structure and on what means might be appropriate for achieving a reduction in concentration, as discussed in Section VI.

Obtaining an estimate of the annual-average concentration and its uncertainty requires consideration of the various factors just discussed in connection w ith monitoring techniques. Some of these arise directly from the measurement technique itself, so that their effect may be evaluated by contractors or individuals performing measurements. Others require more sophistication, e.g., an analytical approach and sufficient local information to convert a reading from, say, charcoal collection over several days to an estimated annual-average concentration. It is likely that this capability could only be maintained hy a central 
(although, perhans local) authority or contractor. To some extent, such complications can be minimized by careful design of the framework for utilizing the resulting estimate. (See discussion of home transfers, below.)

This interpretive framework is an essential functional element in any local control program. Specifically, it must match a specific measurement result to the structure of guideliniss and yield a course of action, if any. The guidslines presumably have a series of concentration ranges with differing recommendations. These might be ranked as follows: a below-average range in which no consideration of action is suggested, an above-average (but not high) range for which it is suggested only that individuals may wish to consider remedial action, a high range in which action is recommended at some time, a very high range in which action is recommended soon (e.g., within one year), and an extreme range in which action is recommended essentially immediately (e.g., within weeks). Depending on where a measurement result fits into this scheme, either a decision for or against remedial action might be appropriate or subsequent monitoring might be indicated. For example, a charcoalsample result that falls into the lowest range or into the very high range would probably be reliable enough to make a decision, specifically to decline action in the first case and to choose some remedial measure(s) in the second. On the other hand, a charcoal-sample result in the above-average or high ranges would probably warrant further monitoring (i.e., a year-long measurement or a sequence of charcoal measurements) to determine the annualaverage concentration with more assurance, after which a decision could be made on a sounder basis. The details of such a structure for decision require careful consideration. But it is clear such a structure must be adopted, either explicitly or implicitly. The default structure, where no such consideration is given, is simply the one where all measurement results are utilized as though they yield the annual-average concentration with the same degree of assurance. This would be absurd and, where it occurs, leads to unnecessary concern and cost.

A central element in the future for utilizing a diversity of types of measurement results would be simple models or algorithms for obtaining an estimate of the annual-average concentration to which occupants are exposed, utilizing the available results, together with other information, in some consistent theoretical framework. Thus the raw result would be converted to an annual-average concentration utilizing a simplified version of a radonbehavior model, together with specific information on the house type, monitoring protocol, and meteorological conditions, as well as the local experience on temporal and spatial variability of radon concentrations. Such a conversion algorithm - based on the more complex predictive models described in Section III.C - could have a consistent general form for use throughout the nation, but with key parameters and ratios determined by local monitoring results. It would permit the making of an estimate of annual-average concentrations, which - while having significant uncertainties - is still superior to the raw monitoring result. The information in Sections II.B and III.A make clear the inadequacy of using short-term monitoring data alone for making remedial decisions.

\section{Home transfers}

A key problem in radon monitoring and interpretation is the case where the sale of a house is pending and a radon measurement is performed to see if remedial action is "needed," usually by comparison with EPA guidance. This situation affects, not just the individuals involved in the sale, but the real estate industry, and even those involved in employee relocation (see, for example, Mansfield 1986).

In the circumstances of house sales, the real problem is how to arrange the measurement, decision, and action so as to make a decision on remedial action on the basis of either a measurement, or a reasonable estimate, of the annual-average concentration in a living 
space. Two elements suggest themselves as helping accomplish this, one having to do with measurement protocols and interpretations and the other involving disclosure and, where necessary, follow-up and remedial action.

The essential problem in a real estate transaction is that it occurs over a brief period of time. This has of ten resulted in the attempt to determine any need for radon-related remedial action in the same pericd. As a result the monitoring protocol that is, de facto, available to those involved in the transaction is presently only the EPA's "screening" protocol, a short-term measurement in the lowest portion of the house (i.e., the basement for most of the houses in the eastern U.S. that have been monitored). As discussed above, a result from this type of measurement ought not to be compared with the EPA guidance because the screening protocol does not provide a measure of the average concentration to which occupants are exposed. However, as a practical matter, short-term results of ten are compared with the EPA guidance, for lack of anything else, much as the EPA itself of ten does. We have already observed that this can be grossly misleading: a house with 150 $\mathrm{Bq} / \mathrm{m}^{3}(4 \mathrm{pCi} / \mathrm{l})$ of radon in the basement during a short-term winter measurement does not have an elevated concentration, but rather has an average concentration (implying, typically, an annual-average living-space concentration in the vicinity of the average concentration for U.S. houses, i.e., about $50 \mathrm{~Bq} / \mathrm{m}^{3}$ ).

A great improvement would be had by reporting, not just the numerical result, but a corresponding estimate of the annual-average concentration, with an uncertainty band (which ought to be included for short-term measurements in any case, since values can easily vary up or down by a factor of two from one week to the next for such a measurement). Thus, as part of better reporting, instead of a sheet of paper that says "the concentration in your house measured under... conditions was $200 \mathrm{~Bq} / \mathrm{m}^{3 / 1}$ (or $5.4 \mathrm{pCi} / 1$, since SI units are not used by the EPA or contractors), the report would say something like, "based on a short-term measurement result of $6 \mathrm{pCi} / 1$, the estimated annual-average concentration to which occupants of your house are exposed in normal use is $2.5 \mathrm{pCi} / 1$, with a ninety percent chance that the value actually lies between 1 and $5 \mathrm{pCi} / 1 . "$ In this case, in all likelihood the annual-average value for the house is below $150 \mathrm{~Bq} / \mathrm{m}^{3}$, though the actual numerical result from the EPA screening protocol is higher.

Being able to make a reasonable estimate of the actual annual-average (living-space) concentration requires - as discussed above - utilizing the understanding of radon behavior that has been developed over the last decade, and the experience with actual measurement results that is now building up, especially in areas where radon has been found to be a problem. This understanding and experience could be brought to bear to enable people to make sensible decisions about their houses and to minimize the anguish that results from a measurement protocol that, for houses with basements at least, tends to yield a numerical result substantially higher than the concentration that people are exposed to. Furthermore, it would prevent the fear and hysteria caused in an area when it is reported that, say, "29\% of houses exceed the EPA remedial action guideline," when in fact a much smaller percentage do. As part of any revision of guidelines and protocols, it would be a great help if even the EPA screening measurement were revised to place a detector in the actual living space and to report both the numerical resuit and - based on an estimating procedure maintained by local authorities - an estimate of the annual-average concentration. More emphasis also has to be given to the fact that, in ordinary circumstances, a long-term measurement has to follow virtually any short-term measurement.

This brings us directly to the problem faced in real estate transactions. An improved measurement and reporting protocol, and the associated procedure for estimating the annualaverage concentration, would have to be established by authorities outside the real estate community. However, the procedure for using this information sensibly is directly affected by the manner in which transactions occur. As noted above, ordinarily a short-term 
measurement, yie' ding an estimate of the annual-average concentration, would be followed by a long-term measurement if there were a possibility that the real annual-average concentration was above $4 \mathrm{pCi} / \mathrm{l}$. Thus, if the (estimated) concentration, based on a shortterm measurement, is anywhere between, say, 2 and $20 \mathrm{pCi} / 1$, there is a significant possibility that the real annual-average exceeds $4 \mathrm{pCi} / \mathrm{l}$. For estimates greater than something like 10 to $20 \mathrm{pCi} / 1$, one might say that the probability is sufficiently high that, in a real estate transaction, ont might simply presume the level exceeds the guideline and proceed with remedia! action. The problem is that this occurs only in a small number of cases. Usually an estimated level, if higher than average, will bi in the range from 2 to 10 $\mathrm{pCi} / \mathrm{l}$, where there is considerable uncertainty whether the levels actually exceed the guideline. For nost houses with such monitoring results from short-term measurements, the annual-average will not exceed $4 \mathrm{pCi} / 1$, so that the sensible thing is to follow up with a long-term measurement to determine the real average concentration.

This would require that the decision on remedial action be postponed, which becomes possible if the problem is treated as, for example, termite damage is treated in California. Thus the seller could place an amount of money for remedial action in an escrow account, the money to be used if the long-term result indicates that remedial action ought eventually to be performed. If the long-term measurement result turns out to be less than $4 \mathrm{pCi} / \mathrm{l}$, the money would be returned to the seller. This procedure would require development of a transfer "protocol," we might call it, i.e., some kind of agreement attached to the sale, and Da aferably to ne deed, that details what has happened and states what will happen under arious circumstances. Based on the need for disclosure, it is probably useful if reports from radon monitoring be recorded permanently, in any case, independently of whether the result is high or low or whether the house is up for sale. The question at the time of a real estate transaction would then be, not what the radon concentration is or whether remedial action is suggested, but rather how measurements and remediation occur in view of the fact that a sale is being concluded. An amount of money placed in escrow might serve well in this context.

Regardless of the details, one major objective is to make a decision in the light of real information, not a short-term measurement that may or may not indicate the level to which occupants of the house are really exposed. Another major objective is to properiy assuciate any such information with the house in a relatively permanent way, so that any subsequent occupant of the house will know whether measurements were performed and whether the results were high or low. This would then be part of the normal disclosure of information for a house in a high radon area and would remove the onus from any particular house. Furthermore, information on the presence, use, and maintenance requirements of any radon control systems installed also ought to te available for subsequent oc upants.

Such possibilities require examination of modification or additions to the legal instruments used in real estate transactions. Of course, the scope of actions required to lower concentrations is almost always inodest enough that simpler approaches are possible If, for example, the cost is approximately $1 \%$ of the home's selling price, and the prot ility of action being needed is modest (say no more than 10 or 200 s for the area), then the probable cost is so low that remediation is not an issue that even needs to be considered in a real estate transaction. Even if the probability is higher for the area, say, exceeding $20 \%$, the arnount of money involved is so snall that it should not complicate a sale: a $20 \%$ probability of having to spend $\$ 1000$ or $\$ 2000$ is of ten ir-elevant financially, and it appears appropriate to find simple legal approaches for rindling the problem.

In any case, with respect to me zurement protorois and reporting, we can do much betiter than the present situation. Even an approximate estim -tor of the annual-average concentration will be better than lising the present screening result, which we know is of ten complete'y wring for making decisions. Developing this procedure and establishing it as a 
component in public guidance is not the sole responsibility of the real estate industry, but requires the active involvement of regulators and scientists.

The proper interpretation and utilization of monitoring results is a difficu't problem, but exactly the type that is the most important in developing an effective strategy for controlling excessive levels of indoor radon, the subject of Section VI. 


\section{BEHAVIOR OF THE DECAY PRODUCTS}

The health impact associated with radon arises from inhalation of its short-lived decay products. Radon itself, being chemically inert, is not hazardous at environmental levels. However, its decay .oducts are chemically reactive and, upon inhalation, may become doposited on the lining of the lung. Subsequent radioactive decays, notably the alpha particle emissions from ${ }^{218} \mathrm{Po}$ and ${ }^{214} \mathrm{Po}$ - in the case of ${ }^{222} \mathrm{Rn}-$ or ${ }^{212} \mathrm{Bi}$ and ${ }^{212} \mathrm{Po}-$ in the case of ${ }^{220} \mathrm{Rn}$ - damage tissue near the deposition site. It is through this mechanism that the increased risk of lung cancer associated with exposure to radon and its decay products arises.

Although it does not constitute a direct health hazard, radon is a suitable surrogate for characterizing the hazard duc to inhalation of the decay products. The presence of these decay products in indoor air is due to the radioactive decay of radon in indoor air, the rate of whicts is, of course, proportional to the radon concentration. Thus, given that the technology for routinely measuring radon is more developed than that for measuring the decay products, estimating the magnitude of the health risk and making decisions about the need for mitigation are appropriately based on radon concentration data.

Nevertheless, a detailed understanding of the risks of exposure to radon requires knowledge of the factors that govern the concentrations and the physical and chemical states of radon decay products in indoor air. Such knowledge is essential for applying the epidemiological evidence obtained from occupational exposures of underground miners to the problem of estimating risks from indoor exposures of general populations. This knowledge is also needed to evaluate the prospect of reducing the risk of indoor radon exposure without changing the radon concentration by altering the concentrations and/or states of the decay products. Knowledge of radon decay product behavior has application to more fundamental scientific questions as well, including issues of radiolytic particle generation and ultrafine particle dynamics.

In this section, the behavior of radon's decay products is summarized, and references to central works in the literature are provided. Although the discussion of this section focuses primarily on behavior of ${ }^{222} \mathrm{Rn}$ decay products, the same considerations apply in the main to the ${ }^{220} \mathrm{Rn}$ series. At a few points in the discussion, critical differences are indicater

\section{A. General Considerations}

For assessing health risks of radon's decay products, only a subset of the radioactive decay chains are of interest. A species in the decay series following a radon isotope may pose a significant inhalation health hazard if (a) its half life is long enough for its number concentration to build to a significant level; (b) its half life and those of its predecessors are not long relative to biological clearance times; and (c) it emits an alpha particle or it is succeeded by an alpha emitting isotope that satisfies condition (b). Thus, the health hazard from ${ }^{222} \mathrm{Rn}$ arises from the alpha decay of ${ }^{218} \mathrm{Po}$ following its inhalation, and from the alpha decay of ${ }^{214} \mathrm{Po}$ following inhalation of ${ }^{218} \mathrm{Po},{ }^{214} \mathrm{~Pb}$, and ${ }^{214} \mathrm{Bi}$. The concentrations and states of ${ }^{218} \mathrm{Po},{ }^{214} \mathrm{~Pb}$ and ${ }^{214} \mathrm{Bi}$ determine the magnitude of this hazard. Likewise, the magnitude of the health hazard from ${ }^{220} \mathrm{Rn}$ is determined by the concentrations and states of ${ }^{212} \mathrm{~Pb}$ and ${ }^{212} \mathrm{Bi}$; the inhalation of either isotope leads to an alpha decay from either ${ }^{212} \mathrm{Bi}$ or ${ }^{212}$ Po (see Appendix D).

One overall measure of the health hazard of the decay products is given by the potential alpha energy concentration (PAEC) or, analogously, the equilibrium-equivalent decayproduct concentration (EEDC). The relationship between the activity concentrations of the 
constituent isotopes and these lumped measures are defined in Appendix A.

Because they convey no information about the physical or chemical state of the decay products, the EEDC and PAEC are imperfect measures of the radon-related health hazard. The most important parameter assciciated with the state of a decay product is its diffusion coefficient, which is a measure of its ability to migrate across fluid streamlines and deposit onto surfaces, including those within the lung. A major distinction is drawn between the decay products that are attached to preexisting airborne particles, and those that are not so attached, referred to as "attached" and "unattached," respectively. The partitioning between these states is commonly reported by means of the "unattached fraction," which is the ratio of the concentration of unattached decay products to the total concentration of decay products. The unattached fraction may be applied to an individual isotope, such as ${ }^{218} \mathrm{Po}$, or to an aggregate measure, such as the EEDC. For the attached fraction, the diffusivity is determined by the size of the airborne particles to which the decay products are attached. For the unattached fraction, the diffusivity is largely determined by the degree of clustering of water vapor and other low-vapor-pressure gases around the decay product.

Because of its smaller size and correspondingly greater diffusivity, a much larger radioactive dose to the bronchial epithelium is ascribed to the unattached decay products $(100-150$ $\mathrm{mGy} / \mathrm{WLM}$ ) than to those that are attached (5-20 mGy/WLM) (James 1988). ${ }^{4}$

Apart from its size, the electric charge associated with a decay product influences the probability or rate of its deposition. Migration of charged species in response to electric fields may alter the rate of deposition of radon decay products onto indoor surfaces. For the unattached fraction, the diffusion coefficient of the charged species is smaller than that of the corresponding neutral species (Phillips et al. 1988). In any case, neutralization is rapid, so that only a small portion (order of $10 \%$ ) of the unattached ${ }^{216}$ Po present in the air is in a charged state (Jonassen and McLaughlin 1988).

In an atmosphere in which the concentration of radon is stable and each decay product, once formed, is removed only by radioactive decay, radon and its decay products would achieve a state of radioactive equilibrium, with each having the same (radioactivity) concentration. For buildings, in which indoor air is exchanged with outdoor air at some rate, not only is the radon concentration less than it would otherwise be, but the concentrations of the decay products are reduced relative to that of radon because of removal by ventilation.

However, the behavior of the decay products, as suggested above, is further complicated by their chemical activity: the fact that the decay products can attach to particles or surfaces, and that these attachment rates can vary with conditions, makes general characterization of the state of the decay products, and its dependence on governing factors, complex. Nevertheless, since we are dealing with only a few species, whose rate of production from early members of the decay chain is determined solely by known half lives, it is possible to specify a relatively straigntforward framework for considering the behavior of the decay products. The specification of this framework is the subject of the next section.

\section{B. The Jacobi Model}

Figure 19 illustrates, for an unspecified decay product, or "progeny," various mechanismis for changing the state or presence of the decay product, other than radioactive decay itself. Given the parameters that are indicated in Figure 19, a system of conservation-of-activity

4 One $G y$ represent the deposition of $1 \mathrm{~J}$ of energy from ionizing radiation per $\mathrm{kg}$ of tissue. The WLM is a measure of decay-product exposure, as discussed in Appendix A. 
Other Removal Processes:

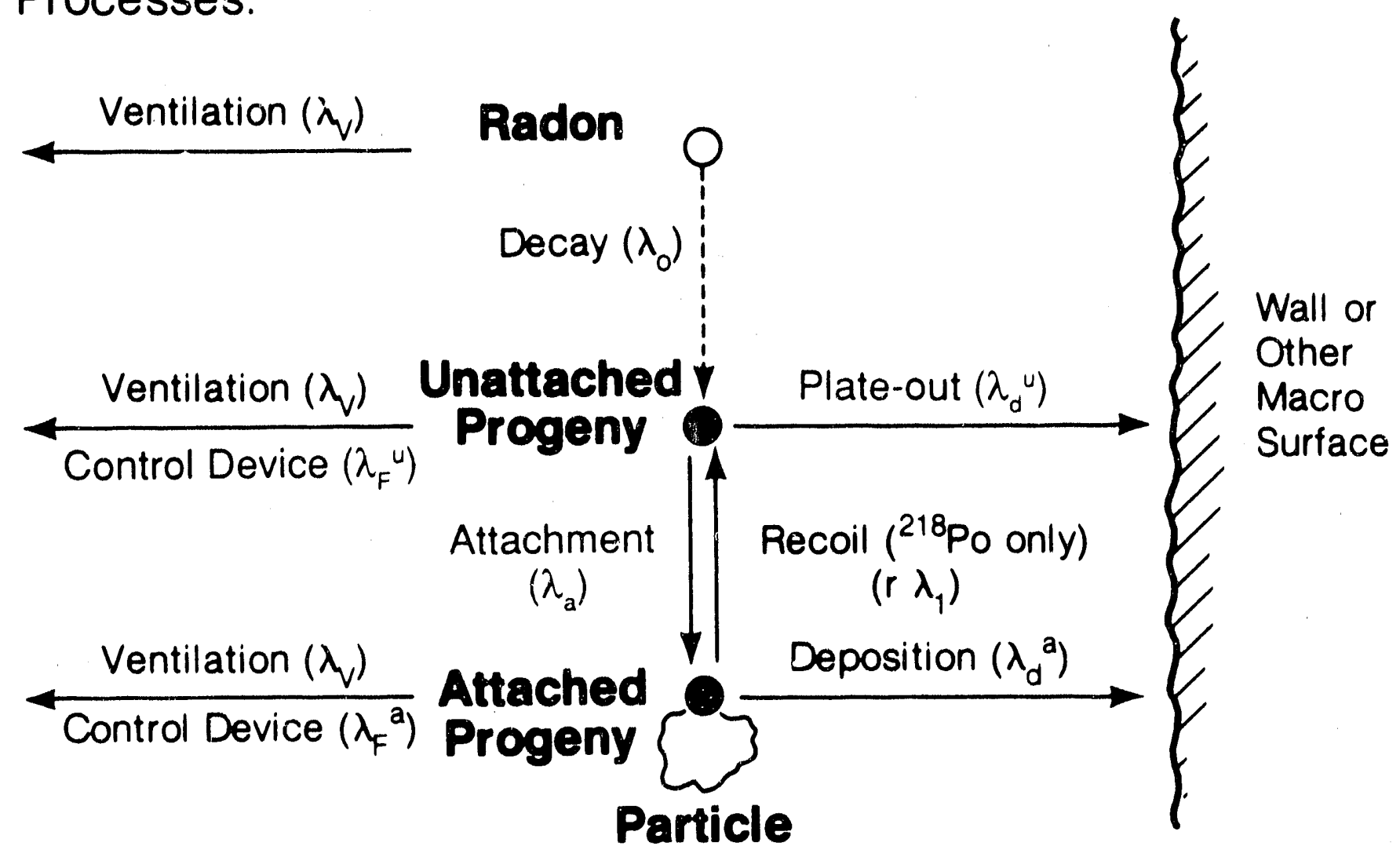

Figure 19. Decay-product removal mechaninisms (other than radioactive decay) and associated rate constants.

Once created by decay of its parent, a decay product (or "progeny") may attach to an airborne particle, a process that is usually considered to be reversible for ${ }^{218}$ Po because of the substantial recoil energy associated with alpha decay. Whether attacined or not, a decay product can be removed from the indoor air by plateout/deposition on indoor surfaces, by ventilation, by an air-cleaning device, or by radioactive decay. 
equations can be specified that determine the concentrations based on given rate constants (Jacobi 1972; Porstendorfer et al. 1978; Knutson 1988). Practical application of such a theoretical framework usually requires assumptions that simplify the picture. For example, mixing within the indoor space s of ten considered to be occur sufficiently rapidly that the concentration of each speritur. Is independent of position, except in a thin boundary layer adjacent to each surface alsi, it is commonly assumed that rate constants for attachment and deposition are indepe $x_{\text {and }}$ of the specific species. The model is then stated as a set of algebraic equations reflecting the balances of steady-state concentrations among attached and unattached species, as follows (Knutson 1988):

$$
\begin{aligned}
& \lambda_{1} A_{0}=\left(\lambda_{1}+\lambda_{v}+\lambda_{a}+\lambda_{d}{ }^{u}\right) A_{1}{ }^{u} \\
& \lambda_{a} A_{1}{ }^{u}=\left(\lambda_{1}+\lambda_{v}+\lambda_{d}{ }^{a}\right) A_{1}^{a} \\
& \lambda_{2} A_{1}{ }^{u}+r \lambda_{2} A_{1}^{a}=\left(\lambda_{2}+\lambda_{v}+\lambda_{a}+\lambda_{d}{ }^{u}\right) A_{2}{ }^{u} \\
& \lambda_{a} A_{2}{ }^{u}+(1-r) \lambda_{2} A_{1}^{a}=\left(\lambda_{2}+\lambda_{v}+\lambda_{d}{ }^{a}\right) A_{2}{ }^{a} \\
& \lambda_{3} A_{2}{ }^{u}=\left(\lambda_{3}+\lambda_{v}+\lambda_{a}+\lambda_{d}{ }^{u}\right) A_{3}{ }^{u} \\
& \lambda_{3} A_{2}{ }^{a}+\lambda_{a} A_{3}{ }^{u}=\left(\lambda_{3}+\lambda_{v}+\lambda_{d}{ }^{a}\right) A_{3}{ }^{a}
\end{aligned}
$$

In these equations, $A_{0}$ represents the activity concentration of ${ }^{222} \mathrm{Rn}, \mathrm{A}_{i}{ }^{\mathrm{a}}$ and $\mathrm{A}_{i}{ }^{\mathrm{u}}$, represent, respectively, the attached and unattached activity concentrations of the ith decay product, $\lambda_{i}$ is the radioactive decay constant of the ith decay product, $\lambda_{y}$ is the removal rate by ventilation, $\lambda_{\mathrm{a}}$ is the rate of attachment to preexisting particles, and $\lambda_{d}{ }^{\mathrm{a}}$ and $\lambda_{d}{ }^{\mathrm{u}}$ represent the deposition rate of attached and unattached decay products, respectively. The isotopes ${ }^{218} \mathrm{Po},{ }^{214} \mathrm{~Pb}$, and ${ }^{214} \mathrm{Bi}$, correspond to $\mathrm{i}=1,2$, and 3 , respectively. The parameter $\mathrm{r}$ denotes the probability that, on alpha decay, the newly formed ${ }^{214} \mathrm{~Pb}$ atom will become detached by recoil from the particle to which its predecessor was attached. (Detachment is not considered to occur with the less energetic recoil resulting from the beta decay of ${ }^{214} \mathrm{~Pb}$.) In this formulation, the removal rate of decay products by filtration is assumed to be zero.

This set of equations contains eight parameters (seven rates $\lambda$ and the recoil probability $r$ ). If values for these parameters are known, then the attached and unattached concentrations of each of the short-lived ${ }^{222} \mathrm{Rn}$ decay products (six unknowns) can be computed from this model (six equations) for any given ${ }^{222} \mathrm{Rn}$ concentration. Conversely, as is of ten done, the model can be used with measurements of the decay product concentrations to determine values of the parameters.

In the next three sections, evidence pertaining to the value of these paraneters in rooms is discussed briefly. Knutson (1988) provides a more detailed examination and evaluation of the data.

\section{Radioactive Decay, Recoil, and Ventilation}

The probability of an isotope radioactively decaying varies greatly from one species to another, but, for a given species, is invariant with respect to changes in environmental conditions. The probability of decay is commonly given in terms of a decay constant, which represents, for a system in which no new prociuction occurs, the siupe of the natiurá logarithm of the amount of an isotope vs. time. For the short-lived decay products of ${ }^{222} \mathrm{Rn}$, the decay constants are $13.7 \mathrm{~h}^{-1}$ for ${ }^{218} \mathrm{Po}$ (Martz et al. 1989), $1.55 \mathrm{~h}^{-1}$ for ${ }^{214} \mathrm{~Pb}$ and 
$2.09 \mathrm{~h}^{-1}$ for ${ }^{214} \mathrm{Bi}$ (Browne and Firestone 1986).

Polonium-218 decays by emitting an alpha particle, i.e., two protons and two neutrons, from its nucleus with a kinetic energy of $6.0 \mathrm{MeV}$. The newly formed ${ }^{214} \mathrm{~Pb}$ atom recoils much as a gun recoils from the firing of a bullet. Its recoil energy is $110 \mathrm{keV}$, given by $\left(\mathrm{m}_{\alpha} / \mathrm{m}_{\mathrm{Pb}}\right) \mathrm{E}_{\alpha}$ where $\mathrm{m}_{\alpha}$ and $\mathrm{E}_{\alpha}$ are the mass and kinetic energy, respectively, of the alpha particle, and $m_{p b}$ is the mass of the lead atom. This energy is sufficient to break chemical bonds so that, if the ${ }^{218}$ Po was attached to a particle, there is a significant probability that the ${ }^{214} \mathrm{~Pb}$ atom will become detached on formation. In the model described in the previous section, the parameter $r$ accounts for this process. Mercer (1976) recommends a value of 0.83 for this parameter, and this value will be used here.

Note that even though a beta decay may be accompanied by the relesise of energy of similar magnitude to that of an alpha decay, the recoil of the atom formed by beta decay is negligible, owing to the small mass of the electron. The maximum recoil energy for ${ }^{214} \mathrm{Bi}$ from beta decay of ${ }^{214} \mathrm{~Pb}$ is $2.6 \mathrm{eV}$, almost 5 orders of magnitude smaller than the recoil energy of ${ }^{214} \mathrm{~Pb}$. Thus, recoil detachment of ${ }^{214} \mathrm{Bi}$ is not considered in the model.

Ventilation rates in buildings span a considerable range. For residential buildings, the ventilation rate is most of ten reported as the air flow rate divided by the building volume, with units of inverse hours. A typical value is $0.5 \mathrm{~h}^{-1}$, and availavi: data suggest that the central $90 \%$ of the distribution is in the range $0.2-2 \mathrm{~h}^{-1}$ (Nazaroff et al. 1987b). Further discussion of ventilation can be found in Appendix B.

\section{Attachment}

Ambient air, whether outdoors or inside buildings, contains a surprisingly high concentration of suspended particles. The size of these particles varies from molecular clusters having a diameter in the vicinity of $0.001 \mu \mathrm{m}$ to dust particles and pollens that are several tens of $\mu \mathrm{m}$ in diameter. Most of the aerosol mass is found in two narrower size ranges: a fine mode covering the approximate range 0.05 to $2 \mu \mathrm{m}$ diameter, and a coarse mode from 2 to about $50 \mu \mathrm{m}$ in ciameter.

Unattached radon decay products, and particles, migrite in air because of random collisions with surrounding gas molecules. Through this frocess (among other, generally less important processes) the unattached decay product may collide with a preexisting particle and adhere, becoming an attached decay product. For a given atmosphero, the rate at which attachment occurs depends on the number concentration of particles, and on their size distrit tion. The total rate of attachment is given by the relationship

$$
\lambda_{a}=\int_{0}^{\infty} \beta\left(d_{p}\right) n\left(d_{p}\right) d\left(d_{p}\right)
$$

where $d_{p}$ is the particle diameter, $\beta\left(d_{p}\right)$ is the attachment rate coefficient, and $n\left(d_{p}\right)$ is the size distribution density function, which satisfies

$$
N=\int_{0}^{\infty} n\left(d_{p}\right) d\left(d_{p}\right)
$$

where $\mathrm{N}$ is the total number concentration of particles. For particles that are small relative to the mean-free-path of air molecules $(\zeta=0.065 \mu \mathrm{m})$, the attachment rate coefficient is proportionai to the square of the particie diameter; for iarge particies, the attacinment rate coefficient is directly proportional to the particle diameter. An expression that spans both 
conditions is cited by Phillips et al. (1988):

$$
\beta\left(d_{p}\right)=\frac{2 \pi \bar{V} d_{p}{ }^{2}\left(d_{p}+2 \zeta\right) D}{8 D\left(d_{p}+2 \zeta\right)+\bar{V} d_{p}{ }^{2}}
$$

where $\bar{V}$ is the mean thermal velocity of the unattached decay products $\left(\sim 140 \mathrm{~m} \mathrm{~s}^{-1}\right)$ and $D$ is the diffusion coefficient of the unattached decay products $\left(\sim 0.05-0.06 \mathrm{~cm}^{2} \mathrm{~s}^{-1}\right)$. Measurements of the activity-weighted particle size distribution show that most of the attached decay products are associated with particies in the diameter range 0.05 to $0.3 \mu \mathrm{m}$ (George and Breslin 1980).

Attachment rates in the range 3 to $600 \mathrm{~h}^{-1}$ have been calculated for indoor environments by combining expressions like these with measurements of the particle size distribution (e.g., Raes et al. 1984). A simpler approach is to combine a characteristic mean value for $\beta$ with a measurement of the total number concentration of particles (as condensation nuclei) in the air (Knutson 1988). Values for $\bar{\beta}$ in the range $(2-8) \times 10^{-3} \mathrm{~cm}^{3} \mathrm{~h}^{-1}$ have been reported by Porstendorfer (1984). The accuracy of the latter approach depends on the consistency of the particle size distribution, a condition that may hold approximately, but certainly not rigorously.

On the basis of available data, Knutson (1988) suggested that attachment rates for indoor environments typically are within the range 5 to $500 \mathrm{~h}^{-1}$, which corresponds to a particle number (condensation nucleus) concentration in the range $10^{3}$ to $10^{5} \mathrm{~cm}^{-3}$, given an average attachment rate coefficient of $5 \times 10^{-3} \mathrm{~cm}^{3} \mathrm{~h}^{-1}$. A "baseline" value for attachment of $50 \mathrm{~h}^{-1}$ is suggested, corresponding to the geometric mean of the end points of this range. Note that through the use of high efficiency filters, particle number concentrations may be reduced to levels substantially lower than $10^{3} \mathrm{~cm}^{-3}$ (Offermann et al. 1985).

Given the broad range of values it may span, the attachment rate is p-tentially important as a parameter affecting the dose associated with a given radon level. More information on the number concentration and size distribution of particles in indoor air would improve the understanding of radon health risks.

\section{E. Deposition}

As depicted in Figure 19, radon decay products, either attached or unattached, may become deposited onto surfaces in a room. This process reduces the airborne concentration and therefore the dose associated with a given radon level.

In general, the deposition rate of reactive pollutants onto indoor surfaces varies with the nature and strength of near-surface flows (Nazaroff and Cass 1989a). Turbulent flow conditions and higher nedr-surface velocities yield smallir boundary-layer thicknesses and consequently higher deposition rates. The deposition rate also varies with the diffusion coefficient of the species, and so radon decay products that are unattached deposit at a much higher rate than do those that are attached.

The "deposition velocity" is a useful concept for reporting theoretical and experimental deposition rates. Denoted by $v_{d}$, it is defined in this context as the decay-product flux density to a surface (units: activity per area per time) divided by the concentration (units: activity per volume) at a large distance from the surface. Thus, $v_{d}$ has units of velocity. The total loss rate due to deposition, $\lambda_{d}{ }^{a}$ or $\lambda_{d}{ }^{u}$ for attached or unattached decay products, respectively, is given by $v_{d} A / V$, where $A$ is the surface area of the room, $v$ is its volume, and the deposition velocity is averaged over all surfaces. 
Measurements of the deposition velocity of unattached radon decay products have been made by several investigators. Unfortunately, many of these measurements have been carried out in experimental chambers in which the flow conditions were not characterized, and so these results cannot be used to predict deposition rates in rooms. However, measurements have been made in a commercial building (Scott 1983), in a utility room of a house (Toohey et al. 1984) and in a $26 \mathrm{~m}^{3}$ chamber (Bigu 1985). The resulting deposition velocities are in the range $6 \times 10^{-4}$ to $53 \times 10^{-4} \mathrm{~m} \mathrm{~s}^{-1}$, with $22 \times 10^{-4} \mathrm{~m} \mathrm{~s}^{-1}$ recommended by Knutson (1988) as a representative average over the surfaces of a typical room. Given a characteristic value of $2.5 \mathrm{~m}^{-1}$ for $A / V$, the baseline deposition velocity recommended by Knutson corresponds to a loss rate for unattached radon decay products of $20 \mathrm{~h}^{-1}$. Rudnick and Maher (1986) measured a deposition velocity of $23 \times 10^{-4} \mathrm{~m} \mathrm{~s}^{-1}$ for unattached decay products in a $78 \mathrm{~m}^{3}$ chamber. With mixing fans in operation, the deposition velocity was increased to the range $(92$ to 130$) \times 10^{-4} \mathrm{~m} \mathrm{~s}^{-1}$.

Recent advances in modeling of air movement have made it possible to begin removing the simplifying assumption ordinarily used for simulation of decay-product behavior, that of a well-mixed room. The more detailed formulation permits treatment of the boundary layer more realistically, thereby providing a basis for determining the manner in which plateout rate (or deposition velocity) depends on conditions in the room, especially near the wall (Schiller et al. 1984, Schiller 1984).

These experimental results are higher than can be accounted for under natural convection flow conditions, but within the range that might result from turbulent flow in rooms (Nazaroff and Cass 1989a). Too little information is available on air flow in occupied buildings, and especially in residences, to reliably predict deposition rates of unattached radon decay products for specific conditions.

The deposition rate is substantially smaller for attached decay products because of their large: associated mass. Experiments and theoretical predictions suggest deposition velocities in the range $10^{-6}$ to $3 \times 10^{-5} \mathrm{~m} \mathrm{~s}^{-1}$ for $0.1 \mu \mathrm{m}$ diameter particles (Nazaroff and Cass 1989a). The baseline loss rate of attached decay products by deposition recommended by Knutson (1988), $\lambda_{d}{ }^{a}=0.2 \mathrm{~h}^{-1}$, corresponds to a mean deposition velocity of $2.2 \times 10^{-5}$ $\mathrm{m} \mathrm{s}^{-1}$, given $\mathrm{A}^{\prime} \mathrm{V}=2.5 \mathrm{~m}^{-1}$.

\section{F. Implicutions for Health Effects}

From the information presented in this section, the relationship between indoor radon concentration and radiological dose to the bronchial epithelium resulting from inhalation of radon's decay products can be evaluated. We consider the dose from occupying a residence $75 \%$ of the iime. The indoor radon concentration is taken to be $55 \mathrm{~Bq} \mathrm{~m}^{-3}$, the mean $\mathrm{fnr}$ U.S. single-family dwellings (Ser:tion II.A).

The parameter values in the Jacobi model are taken from Knutson (1988), with low-dose, and high-dose conditions representing the limits of the typical range. The relationship between exposure and dose is based on the work of James (1988). It should be recognized that information on nasal deposition and cells at risk for cancer is continuing to develop, slightly altering the basis for dosimetric calculations, as discussed by James (1989a and 1989b). Still, his earlier (1988) results are indicative of the size and magnitude of the dose from unattached and attached decay products, and have been used in our calculations.

The calculation results are presented in Table 6. For baseline conditions, the dose resulting from exposure $75 \%$ of the time to $55 \mathrm{Ba} \mathrm{m}^{-3}$ in a residence is $3.1 \mathrm{mGy}^{-1}$. The corresponding effective-dose equivalent is $3.7 \mathrm{mSv}^{-1}$, substantially higher than mean value 
Table 6. Mean bronchial epithelial dose due to indoor ${ }^{222} \mathrm{Rn}$ decay-product exposure. ${ }^{\mathrm{a}}$

\begin{tabular}{lccccccc} 
Condition & $\lambda_{\mathrm{v}}\left(\mathrm{h}^{-1}\right)$ & $\lambda_{\mathrm{a}}\left(\mathrm{h}^{-1}\right)$ & $\lambda_{\mathrm{d}^{\mathrm{a}}\left(\mathrm{h}^{-1}\right)}$ & $\lambda_{\mathrm{d}^{\mathrm{u}}}\left(\mathrm{h}^{-1}\right)$ & $\begin{array}{c}\text { EEDC } \\
\left(\mathrm{Bq} \mathrm{m}^{-3}\right)\end{array}$ & $\mathrm{f}_{\mathrm{p}}(\mathrm{b})$ & $\begin{array}{c}\text { Dose } \\
\left(\mathrm{mGy}^{-1}\right)\end{array}$ \\
\hline Low-dose & 1.5 & 500 & 0.4 & 40 & 18.2 & 0.011 & 1.6 \\
Baseline & 0.5 & 50 & 0.2 & 20 & 20.9 & 0.062 & 3.1 \\
High-dose & 0.2 & 5 & 0.1 & 10 & 14.1 & 0.32 & 6.5 \\
\hline
\end{tabular}

a Based on $75 \%$ occupancy at the mean concentration of $55 \mathrm{~Bq} / \mathrm{m}^{3}$ for U.S. single-family dwellings. The decay product concentrations are calculated usirg the Jacobi model (Jacobi 1972; Knutson 1988). The dose conversion factors are $125 \mathrm{mGy} / \mathrm{WLM}$ for unattached decay products and $7.5 \mathrm{mGy} / \mathrm{WLM}$ for attached decay products (James 1988). These factors are sensitive to the presumed behavior of the decay products in the nasal passages and to what epithelial cells are considered to be potential sites for aiterations leading to cancer. Some changes in these presumptions have occurred since the calculations of James (1988), as indicated in James (1989a). New information is still being developed, causing some changes in the doses given in this table, but these changes are only on the order of 10 or $20 \%$ (James 1989b).

b Unattached fraction of the potential alpha energy concentration or, equivalently, of the EEDC. 
of $1.4 \mathrm{mSv} \mathrm{y}^{-1}$ associated with other radiation exposures (Sinnaeve et al. 1984). (Note: the dose equivalent computed here for residential radon exposure is much higher than the 1 $\mathrm{mSv} \mathrm{y}^{-1}$ reported by Sinnaeve et al.)

The radiation dose associated with a given indoor radon level varies by a factor of 4 from "low-dose" to "high-dose" conditions. Note that the dose correlates with the unattached fraction, but not with the EEDC. This observation is consistent with the findings by James (1988) that the ${ }^{222} \mathrm{Rn}$ concentration may be as good as (and in many cases a better) indicator of effective-dose equivalent than the EEDC.

\section{G. Effect of Air-Cleaning Devices}

The effect of operating an air-cleaning device on the radiological dose from radon's decay products is not easily evaluated. Passing an air stream through a high-efficiency particle filter, for example, would effectively remove both attached and unattached decay products, reducing dose. However, operation of such a device in an indoor environment would also reduce the concentration of airborne particles, thereby lowering the attachment rate $\lambda_{a}$, and increasing the unattached fraction, possibly increasing dose. To complicate matters further, the operation of a control device of ten increases the degree of air movement in a room which would lead to higher rates of deposition, again reducing dose. Certain air cleaners, some ionizers for example, alter the electric field strength in a room which in turn may affect the deposition rate of attached and unattached decay products, and non-radioactive particles. Thus, quantifying the benefit that might be achieved through the use of an air cleaner in an indoor atmosphere is complicated.

The Jacobi model, coupled with a dosimetric model, as described in previous subsections, can serve, with modification, as a framework for evaluating the effectiveness of aircleaning devices (torassen and McLaughlin 1988). One need only (1) interpret $\lambda_{v}$ to be the total rate of removal by ventilation and filtration, and (2) properly account for the effect of the control device on the parameters $\lambda_{a}, \lambda_{d}{ }^{a}$, and $\lambda_{d}{ }^{4}$. Of course, it is far from trivial to properly account for changes in the values of these parameters. Changes in the attachment rate, in particular, depend on other considerations such as the rates of supply and removal of indoor particles, a complicated problem in its own regard (Nazaroff and Cass 1989b).

Overall, the radical reduction in decay-product concentration (given as EEDC or PAEC) that is possible by air cleaning with filters may not cause a corresponding decrease in effective dose and in health effects; it is even possible that there is no decrease at all (Jonassen 1984) or an increase (Maher et al. 1987). Recent experimental work by Maher et al. (1987) suggests that combining the use of an ion generator with a mixing fan is an effective method of air cleaning for reducing radiological dose from radon decay products.

\section{H. Recent Research on the Nature and Behavior of Unattached ${ }^{218} \mathrm{Po}$}

The diffusion coefficient of unattached ${ }^{218} \mathrm{Po} i$, a Ley parameter that governs its behavior, affecting its deposition rate, attachment rate, and dose effectiveness. Recent studies on the dynamics of unattached ${ }^{318}$ Po illuminate the factors that influence its diffusivity.

One key facior is the charge state of ${ }^{218} \mathrm{Po}$. Upon decay of ${ }^{222} \mathrm{R} n$ by alpha-particle emission, a recoiling atom of ${ }^{218} \mathrm{Po}$, possessing a kinetic energy of $101 \mathrm{keV}$, is formed. In air, this recoiling atom has a range of $50 \mu \mathrm{m}$, losing its excess kinevic energy over a period of nanoseconds through interactions with gas molecules. At the end of this initial period, most of the ${ }^{218}$ Po atoms possess a single positive charge, with a fraction being electrically neutral. The charged, unattached ${ }^{218}$ Po may be neutralized through one of several 
mechanisms: (a) small ion recombination, the rate of which is proportional to the square root of the radon concentration; (b) electron transfer, which depends on the availability of molecules having a low ionization potential, such as $\mathrm{NO}_{2}$ and $\mathrm{NH}_{3}$; and (c) electron scavenging (Chu and Hopke 1988). The rate of neutralization of ${ }^{218}$ Po thus depends on the constituents of the surrounding atmosphere. Since charged species have a lower diffusion coefficient than corresponding neutral species (Phillips et al. 1988), atmospheric components other than particles affect radon decay-product dynamics.

In addition to the issue of neutralization rate, unattached ${ }^{218}$ Po may serve as a center for the formation of a molecular cluster or an ultrafine particle. The range of experimentally determined diffusion coefficients for unattached ${ }^{218} \mathrm{Po}\left(0.03-0.10 \mathrm{~cm}^{2} \mathrm{~s}^{-1}\right)$ is smaller than the value predicted by kinetic theory for a single, neutral ${ }^{218}$ Po atom (Phillips et al. 1988). In air, ${ }^{218} \mathrm{Po}$ is rapidly oxidized to $\mathrm{PoO}_{2}$. Hopke (1988) reviewed experimental evidence that argues for the production of radiolytic ultrafine particles incorporating ${ }^{218}$ Po by the following mechanism. The recoiling ${ }^{218}$ Po atom causes the dissociation of water vapor molecules into a hydrogen radical and a hydroxyl radical, both highly reactive compounds in air (Seinfeld 1986). Certain trace gases, if present, form low-vapor pressure species upon reaction with hydroxyl radical. One important example is sulfur dioxide, which becomes. $\mathrm{HSO}_{3}$ and is rapidly hydrolyzed to sulfuric acid. Since the recoil path of ${ }^{218} \mathrm{Po}$ is short (order $100 \mu \mathrm{m}$ in air), the low-volatility sulfuric acid molecules would tend to collide with the $\mathrm{PoO}_{2}$ molecule and condense there, rather than on airborne particles. Sulfuric acid is hygroscopic, so water vapor molecules tend to be drawn to the cluster as well. Raes (1985) has developed a framework for theoretical consideration of the cluster formation process, based on thermodynamic arguments.

Such studies (reviewed in Hopke 1988) are improving our fundamental understanding of decay-product behavior. This, in turn, increases our confidence in using the Jacobi framework for assessing the importance and influence of building-related factors and control techniques on the concentrations and physical characteristics of airborne decay products. This framework is a primary basis for estimating the dose to the lung, using dosimetric models such as those presented by NCRP (1984b) and James (1988). 


\section{STRATEGIES FOR CONTROLLING INDOOR CONCENTRATIONS}

During the last decade or so, efforts to characterize the levels of ${ }^{222} \mathrm{Rn}$ decay products in homes have demonstrated that even average indoor concentrations are high enough to entail an unusually large estimated risk compared with other identifiable environmental insults. Furthermore, the higher concentrations found are unacceptable by virtually any standard. A result of these findings has been the sharply growing concern among policy makers and the general public over the radon issue, leading to substantial, sometimes frantic, efforts to identify and control excessive concentrations in areas where these are known to occur.

These recent efforts, along with previous research, have demonstrated a number of difficulties in formulating a program to control indoor radon. One difficulty is the identification of areas or types of buildings where high concentrations tend to occur: possible approaches, including monitoring in samples of houses or examining fundamental parameters affecting radon entry rates, do not as yet yield consistently reliable results. And even when a high-concentration area is identified, finding individual houses with excessive concentrations involves difficult choices in monitoring protocol and interpretation. Even more fundamental is the difficulty of determining what constitutes an acceptable level: existing regulatory structures and building codes give little guidance on the control of pollutants in private environments, so that control efforts have had to rely on makeshift, of ten arbitrary, guidelines. And even when a house is judged to need remedial action, difficulty in deciding what measure(s) might be appropriate is compounded by the sparse and inconsistent experience with various control techniques. Finally, all these difficulties are amplified by a single important point: homes are essentially private environments. The entire process of setting guidelines, finding houses with high levels, and deciding what control techniques are to be employed requires a framework that delineates responsibilities among the concerned parties.

The major elements of a control strategy, and the underlying scientific understanding or technical capabilities are indicated in Figure 20. The purposes of this section are first to examine these essential elements and second to use this framework to explore current $U$. S. federal policy on indoor radon. This examination is intentionally a rather detached view of what might be seen as an urgent problem. But, however important and urgent, indoor radon has been with us since we began to live in enclosed spaces. In the United States, only a few percent of the houses that ought to be considered for remedial action have so far been identified. Careful consideration of basic issues can substantially increase the effectiveness and efficiency of a control strategy that may involve a million homes, millions of individuals, and many billions of dollars.

\section{A. Elements of Control Strategies}

As illustrated in Figure 20, an overall control strctegy consists of three main elements: a system of guidelines, methodologies for identification, and a framework for control. These components are based on an underlying scientific and technical understanding, and - once framed - interact with one another directly.

Before proceeding to a brief discussion of each element, it is useful to indicate these interrelationships somewhat more explicitly, as is done in Figure 21. Several impurtant cbservations can be made at this point:

(1) Each of the "elements" discussed here is, in fact, a system or framework. The system of guidelines would specify several kinds of standards applying to various classes of buildings (e.g., existing or future) and indicating objectives for concentrations or ventilation rates, as 


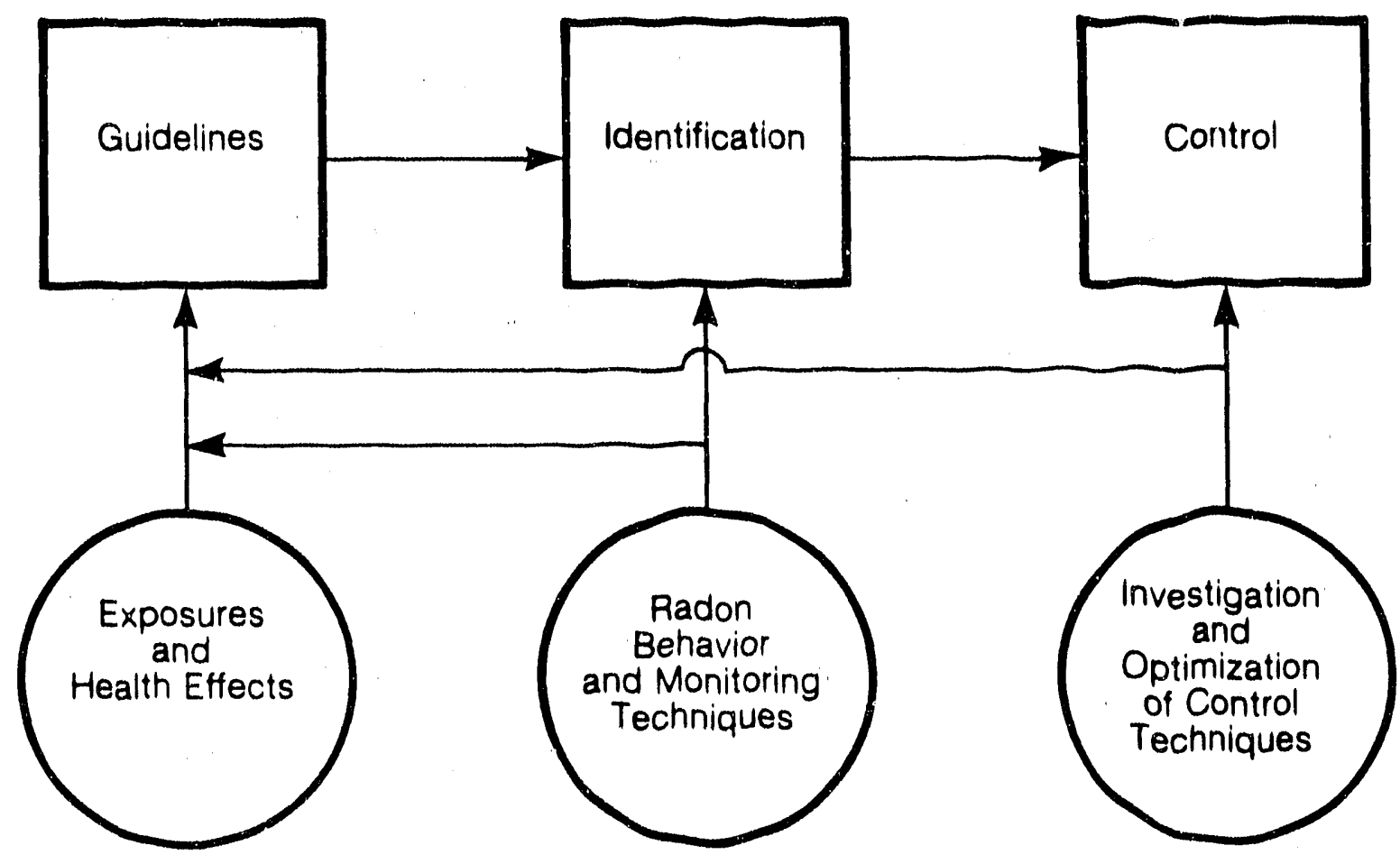

$X B L 871.9205$

Figure 20. The main elements of a control strategy.

These include a system of guidelines, means for identifying the portion of the building stock requiring action, and a framework for choosing appropriate control actions. Formulation of the guidelines and implementation of the control strategy in general depend on scientific understanding of the health risks of indoor exposures, of the behavior of radon and its decay products, and of the principles by which excessive concentrations can be controlled. 


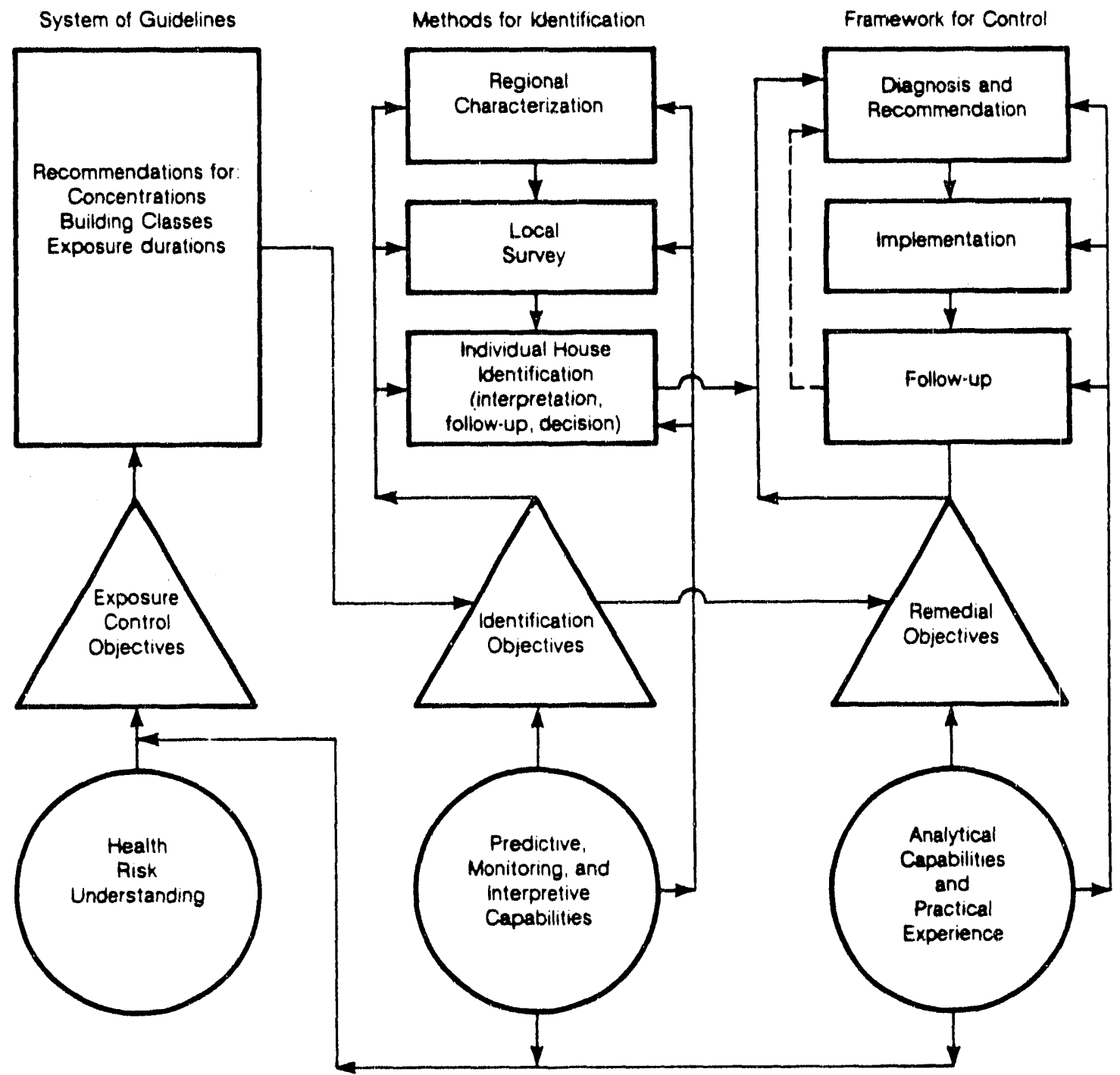

XBL 871.9207

Figure 21. Structure of the main elements of a radon control strategy.

Each of the elements of a control strategy is actually a moderately complex system or framework in itself. In addition, the main elements are joined by a linked set of objectives for exposure control, for identification, and for selection of control measures. The underlying scientific understanding provides support for essential parts of each of the main elements. 
well as time frames for implementation. Similarly, the methodology for identification includes strategies for identifying high-radon areas, for surveying these areas, and for measuring and interpreting concentrations in individual houses. Finally, the framework for control includes a methodology for choosing and implementing appropriate technical measures and for subsequently determining their effectiveness.

(2) Each major element in the strategy is driven by a set of objectives. For the system of guidelines, the goals are set by considerations like those set forth in the next section. In turn, the guidance system, once established, helps to specify the objectives for identification and control. This interrelationship will become evident in the discussion to follow.

(3) The underlying scientific understanding affects the overall control strategy in two important ways. First, it is a basis for determining the objectives for each element. The objectives for the system of guidelines are affected directly by consideration not only of health risks and exposures, but also by the scientific understanding of radon and decay product behavior and by the corresponding design and effectivenes: of methods for identification and control. Secondly, the manner in which the identification methods and control techniques are implemented is guided directly by the general scientific understanding of radon behavior.

The overall framework shown in Figure 21 can be interpreted at two levels. First, it indicates the basic elements needed to implement an overall national strategy for control of indoor radon. Secondly, some key operative elements apply directly to individual structures, i.e., the interpretation of monitoring results and the choice and implementation of appropriate control techniques. It will be noted, as the discussion proceeds, that the problem of finding and alleviating high concentrations in the existing housing stock is emphasized, with particular attention to the case of single-family houses. However, the iramework presented here can be applied to other aspects of the indoor radon question, including future housing, multifamily structures, or buildings devoted to other purposes.

\section{Risk as a basis for specifying objectives.}

One of the principal bases for environmental standards is the potential for the polluiants in question to cause human disease or death. Even for a given setting, it is usually difficult to formulate a consistent set of standards for different pollutants, not only for practical reasons having to do with their origin and cost of control, but also because it is difficult to assess the risk on a uniform basis: pollutants have different types of risks, of ten not comparable, and the quality of the information base used for assessing risk differs from one pollutant to another. This inescapable difficulty is usually compounded by the fact that, from one situation to another, the level of risk that is considered acceptable can change. The indoor environment cannot sensibly be treated on the same basis as either the outdoor or occupational environments, which also have rationales that differ from one another.

To illustrate this point, consider one risk measure - an individual's lifetime risk of early death associated with the following circumstances. Mortality is not, of course, the only risk crite. ion that could be used, but it is suivible for illustrative purposes.

a. Personal criteria for risk aversion tend to cause avoidance of lifetime risks exceeding levels of $10^{-1}$ to $10^{-2}$ if the risks in question are under individual control. The larger number (10\%) is associated with cigarette smoking (which actually has a total mortality risk of $25 \%$ or more) and the smaller $(1-2 \%)$ with automobile accidents. One to a few percent lifetime risk may be the level at which people begin to worry about chronic risks over which they have some control, and - as is clear - even at this level many people will deem the risk acceptable because of some perceived benefit. 
b. Occupational criteria for exposures that are specifically related to the type of work, such as a substance arising from an industrial process, and over which the worker has limited control, tend to lie in the range of $10^{-2}$ to $10^{-3}$. These exposures must be distinguished from incidental ones, such as those that occur merely because a worker is in an indoor space (e.g., an office). It is interesting to note that, except possibly for the recently lowered benzene standard, the lifetime risk associated with ${ }^{222} \mathrm{R} n$ decay-product exposures in mines may be the highest occupational risk from pollutant exposure, several percent according to current risk estimates.

c. Finally, environmental criteria for risks that arise externally to the people exposed, over which they have no control, and that are not directly related to a benefit to them, are typically much less than $10^{-3}$ and of ten in the vicinity of $10^{-5}$, or even $10^{-6}$, a range that the Environmental Protection Agency cornmonly uses as a criterion for examining and regulating environmental risks.

Environmental risks must be distinguished from risks that individuals suffer in circumstances of direct benefit to themselves, specifically in their own homes and places of work. We live indoors because of a wide range of benefits, including comfort, convenience, and health. One might therefore anticipate that appropriate risk criteria for naturally occurring or ocsupantgenerated pollutants in this environment should be in the range between environmental criteria and either occupational or personal criteria. In cases of industrial contamination of living spaces a more stringent standard might be appropriate because of the externality of the insult.

Pollutant exposures cause risks in addition to that of early death. In the indoor environment, emissions from unvented (or improperly operating) combustion appliarces, organic chemicals from a variety of materials and products, and cigarette smoke are of ten found to cause larger exposures to many hazardous compounds than occur in the outdoor environment. These may lead to a significant risk of acute irritant or allergic response or to chronic respiratory illness. It is difficult to compare such risks with that of lung cancer, in terms of either frequency or detriment. Given better knowledge of the full range of risks, one approach is to express all risks in terms of estimated days of life lost or ill, then to assign weighting factors that depend on the severity of the illness.

Simply considering the risk of early death estimated to arise from ${ }^{222} \mathrm{Rn}$-decay-product exposures, the average level of individual risk associated with lifetime exposure (75\% occupancy) at the average concentration of $55 \mathrm{~Bq} \mathrm{~m}$ m $^{-3}$ in $\mathrm{U}$. S. single-family homes is estimated to be $7 \times 10^{-3}(0.7 \%)$ (NAS 1988). As indicated in Section II, some homes have lower concentrations, implying risks in the range of $10^{-4}$ to $10^{-3}$; however, a significant number cause risks exceeding $10^{-2}$ (i.e., $1 \%$ ) and even $10^{-1}(10 \%)$ for lifetime occupancy. It is apparent that a risk criterion in the vicinity of $10^{-5}$ or even $10^{-4}$ is impractical for indoor environments. And it is difficult to conceive of a radon risk criterion as low as $10^{-3}$, not only because it is lower than the present average for indoor environments, but because it is comparable to the estimated risk for continuous exposure to the average outdoor concentration of ${ }^{222} \mathrm{Rn}$ decay products. Even a criterion in the vicinity of $10^{-2}$ would affect a very large number of homes, and have large associated costs, as discussed in Section VI.B.

We inust, therefore, content ourselves with the fact that the risks estimated for indoor exposures to ${ }^{222} \mathrm{Rn}$ decay products will remain much higher than risks associated with most "environmental" insults causing a risk of premature death. We can console ourselves that, by accepting this indoor risk (and others), we gain substantial benefits. On the other hand, it is still important to examine means of avoiding unusually high indoor concentrations, and perhaps even of lowering the average significantly. 


\section{Guidelines and Standards.}

Several classes of standards offer some guidance for the case of indoor radon. Among these are existing radiation standards, general occupational and environmental standards, and building-related standards (Nero 1988b).

Standards or guidelines for controlling indoor radon may be formulated in three ways: (1) by specifying a minimum ventilation rate, thereby controlling the rate at which radon is removed; (2) by requiring the use of radon-resistant construction practices, thereby controlling the rate of radon entry; and (3) by specifying an upper limit for radon concentration. An important example of the first category is ASHRAE Standard 62-1989 on "Ventilation for Acceptable Indoor Air Quality," formulated by the American Society of Heating, Refrigerating, and AirConditioning Engineers. The main orientation of this standard is the provision of a sufficiently high ventilation rate to assure adequately low levels of human-related pollutants, e.g., $\mathrm{CO}_{2}$ and odors. Nonetheless, the basic ventilation rates recommended - approximately 0.5 air changes per hour for homes - also provide adequate assurance of general indoor air quality, assuming that no unusually large pollutant sources are present. Specification of radon-related construction measures for new housing in radon-prone areas might have the broadest effect on public exposures, particularly for houses that would otherwise have decay-product concentrations below $200 \mathrm{~Bq} / \mathrm{m}^{3}(0.05 \mathrm{WL})$. Such measures can be implemented more efficiently in new homes, in the same way that improved specifications on electrical wiring can have a wide effect at low cost in new homes.

Significant steps have been taken in recent years toward development of concentration-based standards for radon in homes. This guidance has been developed primarily for two situations that must be distinguished: (1) remedial action in buildings whose indoor concentrations are thought to be elevated due to industrial activities or contamination and (2) remedial measures in ordinary homes, where the radon comes from sources in their ncicural state or, at least, without elevation due to special types of industrial processing. This report focuses on the latter category; see Nero (1988b) for a discussion of the former.

Considering the recent evidence of a significant incidence of high concenirations in many countries, the radiation protection community has made substantial progress in formulating guidance for ordinary homes (see Table 7). This class of homes is much larger than those with contamination, and the number of people exposed to high concentrations from undisturbed natural sources of radon is very much larger than those seriously affected by industrial residues.

The International Commission on Radiological Protection (ICRP 1984) and the National Council on Radiation Protection and Measurements (NCRP 1984) have made explicit, albeit slightly different, recommendations for radon in buildings. In 1983, the ICRP made recommendations both for remedial action and for new housing, suggesting that, for the first, "if the remedial action considered is fairly simple, an action level in the region of $200 \mathrm{~Bq} / \mathrm{m}^{3}$ $\left(0.055 \mathrm{WL}\right.$, a decay-product concentration equivalent to about $400 \mathrm{~Bq} / \mathrm{m}^{3}$ of $\left.{ }^{222} \mathrm{Rn}\right) \mathrm{might}$ be considered," but that "for severe and disrupting remedial action, a value several times larger might be more appropriate." The NCRP, on the other hand, recommended that remedial action be taken, where necessary, to limit individuals' total exposure to $2 \mathrm{WLM} / \mathrm{y}$. This is equivalent to an average concentration of about $0.04 \mathrm{WL}$ or, assuming as much as $80 \%$ occupancy, an indoor ${ }^{222} \mathrm{Rn}$ concentration of $370 \mathrm{~Bq} / \mathrm{m}^{3}$ (for an equilibrium factor of 0.50 ). Although the NCRP said nothing about new construction, the ICRP did, stating the belief that "a reasonable upper bound [for decay-product concentration] is of the order of $100 \mathrm{~Bq} / \mathrm{m}^{3}$ $(0.027 \mathrm{WL})$ and that, in many countries, a value of this magnitude would prevent radon from becoming a dominating source of risk in dwellings." Although neither of these reports thoroughly considered the causes of and influences on high radon concentrations, both showed substantial sophistication in the formulation of guidance for existing and (in the case of the 
ICRP) future housing.

More recently, the World Health Organization (1987), in recommendations for indoor and outdoor pollutants, dealt with radon and formaldehyde in the indoor environment. The basic recommendation for radon was that remedial actions in buildings with radon concentrations higher than $800 \mathrm{~Bq} / \mathrm{m}^{3}$ be considered in all cases and that the total dose before remedial action should not exceed $4000 \mathrm{~Bq} \mathrm{~m}^{-3}$ y (e.g., that a home with $800 \mathrm{~Bq} / \mathrm{m}^{3}$ should be modified within 5 years). It also recommended that, where simple measures are possible, action be considered for concentrations higher than $200 \mathrm{~Bq} / \mathrm{m}^{3}$. Finally, it recommended that building codes be formulated to avoid levels higher than $200 \mathrm{~Bq} / \mathrm{m}^{3}$ in new structures. This framework of recommendations is similar to the guidance now employed in Sweden, where the relatively high remedial action limit of $800 \mathrm{~Bq} / \mathrm{m}^{3}$ was chosen largely for practical reasons.

During 1986, the EPA issued guidance for U.S. householders (USEPA 1986), recommending that houses with radon concentrations greater than $150 \mathrm{~Bq} / \mathrm{m}^{3}$ be altered to reduce levels. The suggested urgency of the action depends on how high the concentration is, with the speediest action recommended for houses having a radon concentration above $7400 \mathrm{~Bq} / \mathrm{m}^{3}$. The guidelines are stated in terms of radon concentration, a sensible provision considering that the preferred monitoring techniques measure the radon concentration, as discussed in Section IV, and that this appears to be a reliable indicator of risk (cf. James 1988). However, as indicated in Table 7, the recommended remedial action level is lower than that suggested by others, thereby affecting a much larger number of homes.

In 1988 the U. S. Congress passed an amendment to the Toxic Substances Control Act that established, as a long-term national goal, that air in buildings in the United States should be as free of radon as outdoor air (U.S. Congress 1988). The implications of the current EPA recommendations and of the long-term national goal are discussed in Section VI.B.

\section{Identifying houses with excessive concentrations.}

Although a comprehensive strategy for controlling exposures to radon decay products indoors will include attention to the total population exposure, an appropriate near-term focus is on existing homes that have higher-than-average concentrations. Efficient means to identify this portion of the housing stock is therefore an essential element of a cohesive control strategy. A strategy for identifying the housing having high concentrations can be divided practically into two stages: (1) characterization of concentration by area or housing class and (2) intensive efforts in the areas or classes thought to have the highest probability of high concentrations. The first stage can be carried out by a combination of predictive modeling (as discussed in Section III.C) and monitoring (as discussed in Section IV). In this context, predictive modeling is built on an understanding of the factors that govern indoor concentrations and on the availability of data relating to those factors. To use monitoring for this purpose requires an interpretive framework to assess the probability of elevated concentrations in an area or a building class based on the results of a set of measurements.

Two key questions must be addressed in formulating an identification strategy: (1) How many houses are involved? and (2) How are the houses distributed, by location or other characteristics, within the full housing stock?

In view of the apparent frequency distribution of concentrations, the number of houses requiring identification depends critically on the concentration above which remedial action is desired. A change of a factor of two in the remedial action level may cause a change of a factor of five to ten in the total number of houses involved (Nero et al. 1986).

Practical experience combined with systematic examination of the U.S. monitoring data (as 
Table 7. Recommended Limits of ${ }^{222} \mathrm{Rn}$ Concentrations in Buildings ${ }^{\mathrm{a}}$

\begin{tabular}{|c|c|c|c|c|}
\hline & \multicolumn{2}{|c|}{ Existing buildings } & \multirow[b]{2}{*}{$\begin{array}{l}\text { Future } \\
\text { buildings }\end{array}$} & \multirow[b]{2}{*}{ Reference } \\
\hline & Ordinary & Contaminated & & \\
\hline \multicolumn{5}{|c|}{ Professional Organizations } \\
\hline ICRP & $\begin{array}{l}400 \mathrm{~Bq} / \mathrm{m}^{3} \text { (higher level } \\
\text { for more difficult } \\
\text { remedial actions) }\end{array}$ & & $200 \mathrm{~Bq} / \mathrm{m}^{3}$ & ICKP 1984 \\
\hline NCRP & $370 \mathrm{~Bq} / \mathrm{m}^{3} \mathrm{~b}$ & & & NCRP 1984 \\
\hline WHO & $\begin{array}{l}800 \mathrm{~Bq} / \mathrm{m}^{3} \mathrm{c} \\
\left(200 \mathrm{~Bq} / \mathrm{m}^{3} \text { if simple }\right. \\
\text { measures })\end{array}$ & & $200 \mathrm{~Bq} / \mathrm{m}^{3}$ & WHO 1986 \\
\hline ASHRAE & & & $200 \mathrm{~Bq} / \mathrm{m}^{3} \mathrm{e}$ & ASHRAE 1989 \\
\hline \multicolumn{5}{|c|}{ Countries } \\
\hline $\begin{array}{l}\text { United } \\
\text { States }\end{array}$ & $148 \mathrm{~Bq} / \mathrm{m}^{3}$ & $74-370 \mathrm{~Bq} / \mathrm{m}^{3}$ & $\sim 10 \mathrm{~Bq} / \mathrm{m}^{3 \mathrm{f}}$ & $\begin{array}{l}\text { Costle 1979; USEPA } \\
1986 ; \text { U.S.Congress } \\
1988\end{array}$ \\
\hline Canada & & $148 \mathrm{~Bq} / \mathrm{m}^{3}$ & & Létourneau 1986 \\
\hline $\begin{array}{l}\text { Nordic } \\
\text { countries }\end{array}$ & $\begin{array}{l}800 \mathrm{~Bq} / \mathrm{m}^{3} \\
\text { (consider action above } \\
200 \mathrm{~Bq} / \mathrm{m}^{3} \text { ) }\end{array}$ & & $200 \mathrm{~Bq} / \mathrm{m}^{3}$ & RPI 1986 \\
\hline
\end{tabular}

a Recommendations given in terms of EEDC are converted to ${ }^{222} \mathrm{Rn}$ concentrations assuming an equilibrium factor of 0.5 .

b Recommended limit is $340 \mathrm{WL}-\mathrm{h}$ per year, equivalent to $0.050 \mathrm{WL}$ for $80 \%$ occupancy.

c Remedial action to be performed before added exposure exceeds $4000 \mathrm{~Bq} \mathrm{~m}^{-3}$ y $(27$ WLM).

d These countries are Denmark, Finland, Iceland, Norway, and Sweden. In earlier recommendations (Swedjemark 1986), Sweden had specified a $140 \mathrm{~Bq} / \mathrm{m}^{3}$ limit for new buildings and had not specifically recommended consideration of action, in existing buildings, for levels in the range $200-800 \mathrm{~Bq} / \mathrm{m}^{3}$.

e The 1981 ASHRAE recommendation of $74 \mathrm{~Bq} / \mathrm{m}^{3}$ has been changed in a 1989 revision to the equivalent of $200 \mathrm{~Bq} / \mathrm{m}^{3}$ (giving NCRP 1984, apparently incorrectly, as a source for the guideline). However, an addendum to ASHRAE 1989 now being considered would change the ASHRAE recommendation to $148 \mathrm{~Bq} / \mathrm{m}^{3}$, based on USEPA recommendations.

$f$ The U.S. Congress has established a long-term national goal of making air inside buildings as free of radon as outdoor air. 
described in Section II) makes it clear that there is substantial clustering by area of houses with elevated concentrations. This point is illustrated in Figure 22, which shows the cumulative distribution of mean concentrations from 22 geographical areas of the United States (Nero et al. 1986). These means range over more than a factor of ten, even within this small number of areas. (A comparable result based on different data was reported by Cohen (1986).) The individual data sets show that the houses with excessive concentrations tend to be clustered in the areas with mean concentrations that are above the average. Conversely, a large portion of the country appears to have a very small fraction of houses with indoor radon concentrations substantially greater than the nationwide mean. Attention to the area-mean concentration miay thus be regarded as a way of prioritizing the effort, causing the earliest and most substantial attention to be focused on areas that have the bulk of the houses needing remedial action.

Because of the variability of concentrations in any area or class, the final identification of houses for which remedial action should be considered requires direct measurements. A key element in monitoring individual houses will be a framework for interpreting measurement results as a basis for deciding whether action is needed, what kind, and how fast.

\section{Selecting control techniques.}

The presumed situation for choosing remedial measures is one where the results of monitoring, interpreted in light of some set of guidelines, has prompted a decision to seek a reduction in the concentration in a particular house. Along with this specific decision will have come some sense of the importance of the action (reflected indirectly in the degree of concentration reduction sought) and of its urgency (manifested in terms of a recommended time during which action should be implemented). These two objectives are determined in view of monitoring results and the remedial action guidelines, possibly after some diagnosis of potential causes of the high levels, and constitute the broad premises for choosing the course of action and specific measures suited to the house in question. Thus the overall framework for remedial action utilizes objectives designated (at least tentatively) for the house being considered and includes (1) choice of appropriate control measures, (2) a capability for implementing this plan of artion, and (3) any necessary follow-up.

Choosing specific remedial measures in individual homes is the most difficult aspect of operating a remedial action program. The indoor concentration depends in a very complex way on many factors. In particular, the pathways and forces accounting for radon entry into a given house are often extremely complicated and heterogeneous. Due in part to this complexity, as well as to limited experience, procedures for characterizing the specific entry process in a given house are tentative at best. Presently, one can - on the basis of limited examination and measurements - formulate an hypothesis (i.e., a diagnosis). This diagnosis can be tested most efficiently in a very practical way, i.e., by implementing an associated control measure or set of measures. The process of collecting information, formulating a hypothesis, and implementing control measures may be repeated until the desired reduction is achieved.

The apparent success of remedial measures should be confirmed by a follow-up measurement that provides a basis for estimating the new annual-average concentration associated with the house. An apparent change in concentration during the course of remediation cannot be considered sufficient of itself: the apparent success may depend on meteorological or operational conditions that change with time. Moreover, even if the efficacy of the control approach that has been implemented is confirmed by follow-up monitoring, future changes in characteristics of the house structure (e.g., continued settling in the understructure) might cause a later problem. The probability of such difficulties will depend on features of the house being considered and on the type of remedial measures that have been implemented. A control program should incorporate confirmation and follow-up procedures to accommodate 


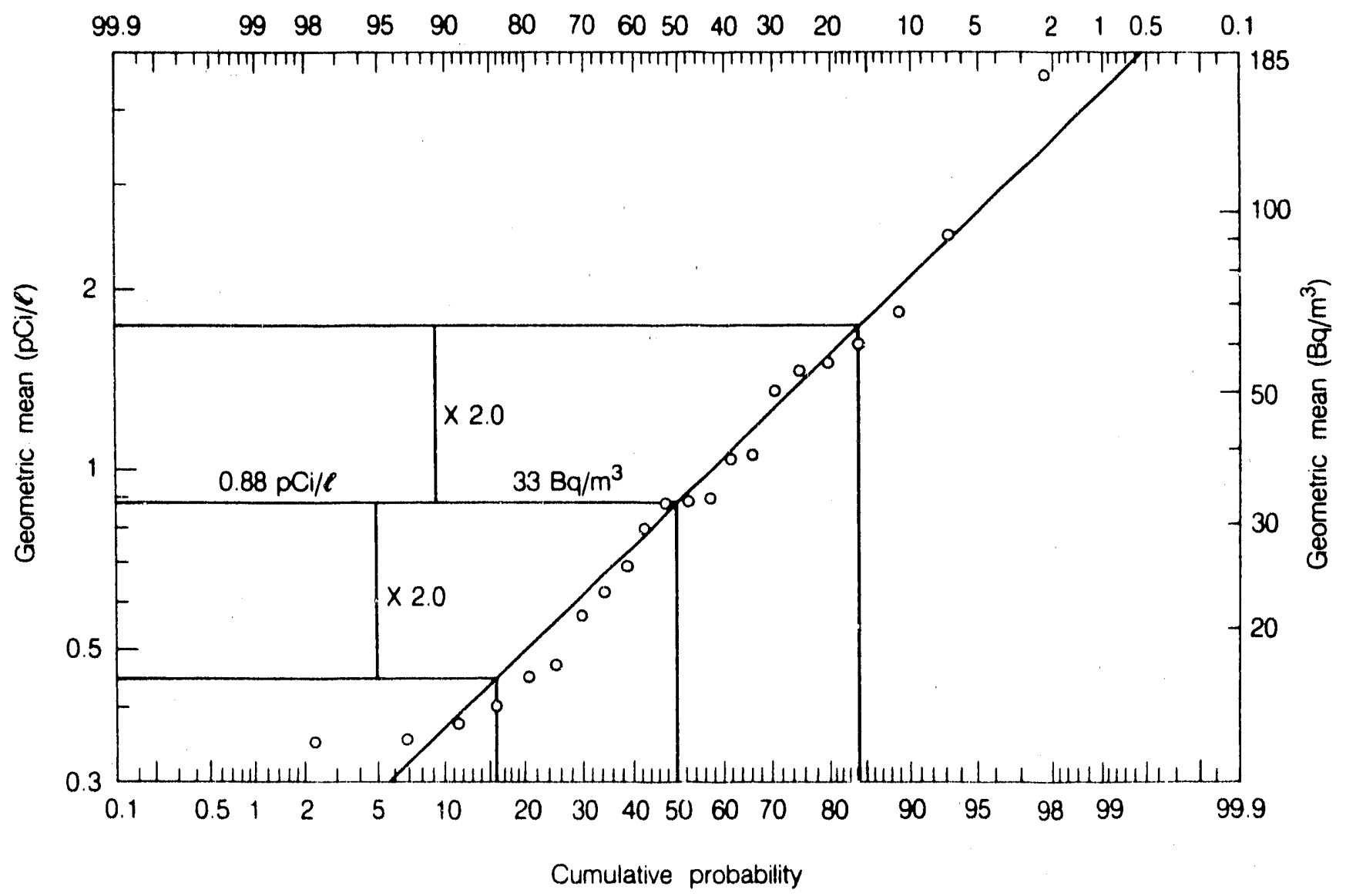

Distribution of annual average GMs for 22 sets of indoor radon data from various areas in the U.S.

X8L 86410770

Figure 22. Distribution of annual-average geometric means for U.S. data.

The geometric means (GMs) from 22 sets of data from around the United States (analyzed by Nero et al. 1986) are plotted versus the probability that the mean is less than the given value. On this scale, if the GMs are distributed lognormally, they should lie along a straight line. The geometric standard deviation of the indicated line is 2.0 , providing a measure of the variability of GMs for these various areas. 
this possibility.

It is useful to illustrate crudely the process of selecting appropriate remedial measures by indicating two of the primary possibilities to be examined and choices to be made. Specifically, one class of choices has to do with the seriousness and urgency of the problem and another has to do with the characteristics of the house.

Considering the first class of choices, if the concentration falls in to the extreme range, something should be done in a short time. The only measures immediately available to most occupants are temporary evacuation, increased ventilation (or perhaps air cleaning using charcoal adsorption), or obtaining the immediate attention of a contractor. None of the options is atiractive. Evacuation is inconvenient and expensive. Sufficient ventilation to effect a large reduction in concentration is of ten uncomfortable and costly (as would be air cleaning at high rates). Securing the rapid attention of a contractor is likely to cause substantially increased costs and, perhaps, decrease the quality of the alterations made. Fortunately, very few houses fall into the range where action is recommended so rapidly. In such cases the optimal choice of ten will be to provide temporary ventilation to reduce the concentration to one of the lower ranges until an attempt at more permanent remedial measures can be arranged.

The more usual situation, i.e., where action is recommended "sometime" or "soon," or where the owner decides to do something even though action is not specifically recommended, will occur with much higher frequency and should be the main focus of a structure for choosing measures. In that case, decisions can be made in light of the relative priority of the factors that affect indoor concentrations. Ordinarily, the ventilation rate in a high-radon house is in the normal range, so that the main focus of control efforts will be on radon entry. (Should this not be true, i.e., if the house is one in which the infiltration rate is unusually low because of special construction practices, then some consideration should be given to increasing the ventilation rate, as discussed below.) Because the main mechanism for radon entry is movement of air from the ground into the house, associated control techniques (Section III.D) seek either to reduce this flow or to reduce the radon concentration in the nearby soil air, or both (cf. Scott 1988). The technique appropriate to a specific house depends on the type of structure, details of the substructure and underlying ground, and the reduction factor sought. The most effective means often have been found to involve systems for altering the pressure balance between the lower part of the house and the ground or for venting radon from the soil to the outdoors. The effectiveness of these systems can be increased by sealing the understructure, even though such sealing of itself is usually relatively ineffective.

Thus, in considering the seriousness of the problem and focusing primarily on the case of moderately high levels, we have turned already to the usual case in terms of house characteristics, i.e., where the overall difficulty is an excessive entry rate and the most likely solution is to be found in reducing flow from the ground. However, there is ? smaller probability of situations where other means are appropriate, either where ventilation is ised on a temporary basis to alleviate truly extreme concentrations, or - somewhat more frequently where the house has much lower than average ventilation rates due to unusual construction practices.

Houses with features that save energy by maintaining unusually low infiltration rates can be expected to have higher-than-average concentrations of radou and other indoor poliutants. Such levels can be reduced by using mechanical systems to increase the overall ventilation rate of the house to a normal level, say $0.5 \mathrm{~h}^{-1}$. The simplest such system is some form of exhaust ventilation. However, because exhaust fans increase the inward pressure across the building shell, they may also increase the rate of radon entry, thus changing the concentration little or even increasing it (Mowris and Fisk 1988). For this reason, systems to increase the house ventilation rate ordinarily should be balanced, incorporating both an air intake and air exhaust. 
(Such a system can utilize heat exchange between the incoming and outgoing air streams, thus avoiding most of the energy loss associated with increased ventilation rates (Fisk and Turiel 1983). Similarly, exhaust ventilation, vtilized for reasons other than radon, can include heat recovery.)

An overall scheme for diagnosis and selection of control techniques must be more detailed than suggested by this overall consideration of urgency, house type, and control approach. It must also be tailored to the type of houses and radon entry modes characteristic of a locale or region. Indeed, even the system of guidelines and the methodologies for survey, monitoring, and interpretation can be expected to depend on the characteristics of the region and the frequency and severity of radon problems in the local building stock. Unfortunately, no concerted effort has yet been mounted, either to examine the risk-limiting objectives or their premises in detail or to develop properly the elements of a radon control strategy.

\section{B. Exploring Policies for Reducing Exposures}

Despite the lack just noted, specific components of a national policy to control exposures to radon decay products in U.S. homes have been put forth in recent years. As indicated in this section, analysis of these components suggests that, at a minimum, billions of dollars would be required to rapidly and fully implement a national radon-control policy. Because of the large associated costs, it is prudent to examine carefully the implications of policy optiors. Two aspects of this issue amplify the importance of such an examination: (1) there are no closely related precedents to the problem of indoor radon from which a rational policy might be adapted; and (2) it is likely that federal policies will be needed to address other indoor air quality isslies in the future.

The purpose of this section is to explore federal policy options for controlling indoor radon. We focus on current policy, examining the number of lung cancer cases that may be avoided, the costs of implementing control measures, and the prospects for attaining the policy goals. Potential problems in identifying and reducing indoor concentrations are considered. Finally, a discission of characteristics that might be expected in a comprehensive policy are presented, emphasizing specific shortcomings of current policy with respect to the general considerations presented in Section VI.A.

\section{Form and implications of current policies.}

Two elements constitute the core of present United States federal policy for controlling exposures to indoor radon. First, the Environmental Protection Agency and the Centers for Disease Control have made recommendations and provided guidance to citizens that include a measurement protocol and guidelines for taking remedial action to limit radon levels in their homes (USEPA 1986; Ronca-Battista et al. 1988; USEPA 1988). Second, an amendment to the Toxic Substances Control Act has recently been adopted that, among other provisions, establishes a long-term national goal of greatly reducing indoor radon concentrations (U.S. Congress 1988). In this section, these policy elements are described, and the cost and reductions in risk associated with successful implementation of the policies are discussed. ${ }^{4}$

\footnotetext{
${ }^{4}$ The Office of Drinking Water of the U.S. EPA is planning to propose a standard to limit radon in public water supplies because of the health risk posed by inhalation of decay products following radon release from the indoor use of water (Federal Register 1986). The costs and potential risk reductions are much smaller than those associated with the EPA/CDC recommendations and TSCA amendment considered in this paper. (On the other hand, the costbenefit ratio may be higher.)
} 
The EPA and CDC have recommended that a screening measurement for indoor radon concentration be made in every household located below a building's third story above ground (USEPA 1988). The measurement protocol calls for a two-day average sample to be taken, under closed-house conditions, on the lowest livable level of the home (USEPA 1986; RoncaBattista et al. 1988). If the measurement result is above $150 \mathrm{~Bq} / \mathrm{m}^{3}(4 \mathrm{pCi} / 1)$, follow-up measurements are recommended to better determine the average concentration to which occupants are exposed. If the follow-up measurements yield an average result in excess of 150 $\mathrm{Bq} / \mathrm{m}^{3}$, it is recommended that corrective action be taken to permanently reduce indoor concentrations. Recommendations on the duration of follow-up measurements and the urgency for corrective action depend on the results of the indoor concentration measurement. The rationale for setting the principal criterion at $150 \mathrm{~Bq} / \mathrm{m}^{3}$ combines historical precedent in the U.S. and the objective of reducing exposures to the extent judged reasonable, given that no exposure can be considered risk-free.

It is important to note that these recommendations do not constitute regulatory standards. According to the present philosophy, it is to be left to individual residents to weigh the risks of radon exposure against the costs of control. In this regard, the strategy for controlling indoor radon departs radically from that for controlling outdoor air pollutants. In the latter case, federal standards have been established, control measures have been implemented, and the imposition of sanctions has been threatened for regions that do not achieve compliance. The differences in approach arise naturally because outdoor air is a community resource, whereas indoor environments are largely private (Sexton and Repetto 1982). However, this position does not accommodate the public resource aspects of the building stock, as discussed in a later section.

We may estimate the cost of a particular strategy by considering the costs of identification (primarily that of monitoring, with present recommendations) and remediation for the number of houses presumed to need it. The estimated cost of implementing the EPA/CDC recommendations in the current housing stock is approximately $\$ 2.2$ billion for measurements and $\$ 6.6$ billion for remediation plus $\$ 0.8$ billion per year for operation and maintenance. ${ }^{5}$ Using an annual discount rate of $5 \%$ with a 30 -year time horizon, the net present value of the costs for measurement and remediation is $\$ 20$ billion.

\footnotetext{
${ }^{5}$ Screening measurements would be required in approximately 80 million residences at a unit cost of $\$ 15$ for a charcoal monitor. Based on the EPA reports that 20-30\% of homes exceed the $150 \mathrm{~Bq} / \mathrm{m}^{3}(4 \mathrm{pCi} / \mathrm{l})$ criterion in screening measurements, subsequent monitoring would be required in approximately 20 millicn residences at a unit cost of $\$ 50$ for two alpha-track monitors capable of measuring long-term average concentrations. Mitigation would be required in $7.3 \%$ of 60 million single-family dwellings at an estimated average cost of $\$ 1500$ for installation, corresponding to the median cost of installing a subslab ventilation system (Turk et al. 1989b). Annual operation and maintenance costs are estimated as $\$ 185$ per remediated household, broken down as follows: $\$ 100$ for electricity to operate the fan (1300 $\mathrm{kWh}$ at $\$ 0.075 / \mathrm{kWh}), \$ 35$ for gas-fired space heating to compensate for additional air exchange $\left(0.2\right.$ air changes per hour in a $300 \mathrm{~m}^{3}$ home located in a 2800 degree-day (centigrade, base 18 C) climate, at $\$ 0.57 /$ therm), nnd $\$ 50$ for annual monitoring, occasional fan replacement, and additional minor maintenance. The cost data apply for homes with basements; less information is available on the ost of reducing radon levels in houses with slab-on-grade or crawl-space substructures.
} 
The benefit in reducing risks depends on the average effectiveness of remediation. If all single-family dwellings having a radon concentration above $150 \mathrm{~Bq} / \mathrm{m}^{3}(4 \mathrm{pCi} / \mathrm{l})$ were reduced to the present average of $55 \mathrm{~Bq} / \mathrm{m}^{3}$, the reduction in total population exposure would be $22 \%{ }^{6}$ If the mitigation measures were less effective, so that the average concentration in remediated housing were $150 \mathrm{~Bq} / \mathrm{m}^{3}$, the reduction in total population exposure would be $12 \%$. The mean of these two results, i.e. reduction to an average of $100 \mathrm{~Bq} / \mathrm{m}^{3}$, is used here for illustration. According to the risk estimates presented in Table 8 for a stable population, complete implementation of the current EPA/CDC policy would reduce the population exposure by $17 \%$, ultimately leading to an annual avoidance of 2500 lung cancer deaths per year - 2300 among smokers and 200 among nonsmokers. An annual lung cancer mortality of more than 12,000 cases would still be attributed to radon exposures.

Assuming a 30-year average effective lifetime for remediated households, the average cost (net present value) per lung cancer death averted under the current guidelines is estimated to be $\$ 0.27$ million. This compares favorably with the range of $\$ 0.4-7$ million per death averted that the EPA considers reasonable to justify the cost of controlling an environmental pollutant (Puskin and Nelson 1989). However, as discussed in Section VI.A.1, the case of indoor radon is not analogous to the situations in which an activity by some external organization is responsible for the hazardous exposure, and public funds are to be spent for remediation. By alternative comparisons, radon remediation is not as attractive. For example, the costs per death averted for installing smoke detectors in homes and for installing passive restraints in automobiles have been estimated at $\$ 30,000$ and $\$ 100,000$, respectively, and yet these riskreducing measures are not uniformly required.

As the guideline for remedial action increases, the average cost-effectiveness of radon control improves. Table 9 shows the results for five action levels applied to single -family dwellings, assuming in each case that remedial measures reduce the average indoor concentration to 100 $\mathrm{Bq} / \mathrm{m}^{3}$, and that the average cost of remediation is independent of the action level. These action levels correspond approximately to the range of values recommended in different countries (Table 7). At the higher guideline levels, most of the cost is asscciated with the screening measurement program, which is assumed to include every home. Mitigation at these higher levels could be made much more cost effective by developing more efficient muans for identifying households with elevated concentrations, e.g., by first identifying areas with high average concentrations, then monitoring intensively in those areas.

For this range of action guidelines, the reduction in population exposure resulting from a successful mitigation program is a small fraction, $0.01-0.17$, of the current exposure. To reduce the current population exposure by a large fraction, remediation would be required in a majority of households, with an action guideline much lower than the current EPA/CDC recommendation. For an action level below $150 \mathrm{~Bq} / \mathrm{m}^{3}(4 \mathrm{pCi} / 1)$, the cost-effectiveness of mitigation is uncertain, but would probably be reduced. A larger fraction of households would require remediation, reducing the average cost per home of identifying those above the

\footnotetext{
${ }^{6}$ Radon concentrations would be reduced from an average of $260 \mathrm{~Bq} / \mathrm{m}^{3}$ to $55 \mathrm{~Bq} / \mathrm{m}^{3}$ in 4.4 million dwellings ( $7.3 \%$ of the 60 million single-family residences), housing 12 million individuals. Assuming average occupancy of $75 \%$, and $50 \%$ equilibrium between radon's decay products and radon, the exposure rate would be reduced by an average of $1.07 \mathrm{WLM} \mathrm{y}^{-1}$ for these 12 million individuals, corresponding to 0.054 WLM $y^{-1}$ or $22 \%$ when averaged over the entire population.

${ }^{7}$ Cost (net present value) per death averted computed as $\$ 20$ billion/(30 years $\times 2500$ cases $\left.y^{-1}\right)=\$ 0.27$ million per case.
} 
Table 8. Estimated annua! lung cancer deaths in the United States attributuble to radon exposure.*

Population

Lung Cancer Death Rate $\left(\mathrm{y}^{-1}\right)$ $1000 \mathrm{~s}$

\author{
All Causes \\ Radon-Attributable
}

Current Population (1986)

Male

never smoked

63,900

1,900

200

former smoker

26,100

42,900

4,800

current, light smoker ${ }^{+}$

18,200

21,400

2,600

current, heary smoker ${ }^{+}$

8,900

22,600

3,100

Female

never smoked

81,300

3,100

300

former smoker

17,100

14,700

1,700

current, light smoker ${ }^{+}$

20,000

15,800

1,900

current, heavy smoke:-

5,300

8,000

1,100

Male, total

117,400

88,800

10,700

123,700

41,600

5,000

Current population, total

241,100

130,400

15,700

Stable Pupulation ++

Male

nonsmoker

82,300

5,900

680

smoker

35,000

67,000

7,700

Female

nonsmoker

$84,: 00$

4,100

470

smoker

39,400

55,000

6,100

Male, total

117,400

73,000

8,400

Female, total

123,700

59,000

6,600

Stable population, total

241,100

132,000

15,000

* Individual entries rounded. For the current population, data were obtained from the following sources: (a) total population by age and sex from 1986 deaths and death rates, U.S. Department of Health and Human Services (1988); (b) population smoking habits by age and sex from U.S. Bureau of the Census (1987); (c) relative risks of death due to lung cancer by age, sex, and smoking habit from Mattson et al. (1987); (d) total lung cancer mortality rates by age and sex from National Center for Health Statistics (1988) Table 8.5. For the stable population calculations, individuals were assumed to either begin a lifelong smoking habit at age 20 or to never smoke. The proportion of males and females at 20 who smoke was taken to be 0.44 and 0.45 , respectively, corresponding to the respective proportions in the age group 20-24 who have ever smoked (reference cited in b above). Separate life tables were constructed by sex and smoking status using 1986 mortality statistics (reference cited in a above) and relative mortality rates by all causes for light smokers (less than 25 cigarettes per day) and persons who have never smoked (reference cited in c above). Lung cancer death rates were assumed to be the same as for the current population. For both conditions, lung cancer mortality rates were corrected for radon-attributable lung cancer incidence to obtain non-exposed incidence rates. Additional references for preparing this table: Nero et al. 1986; NAS 1988.

+ Light smoker defined as one smoking less than 25 cigarettes per day.

++ "Stable" means zero-growth with current age-dependent mortality rates. 


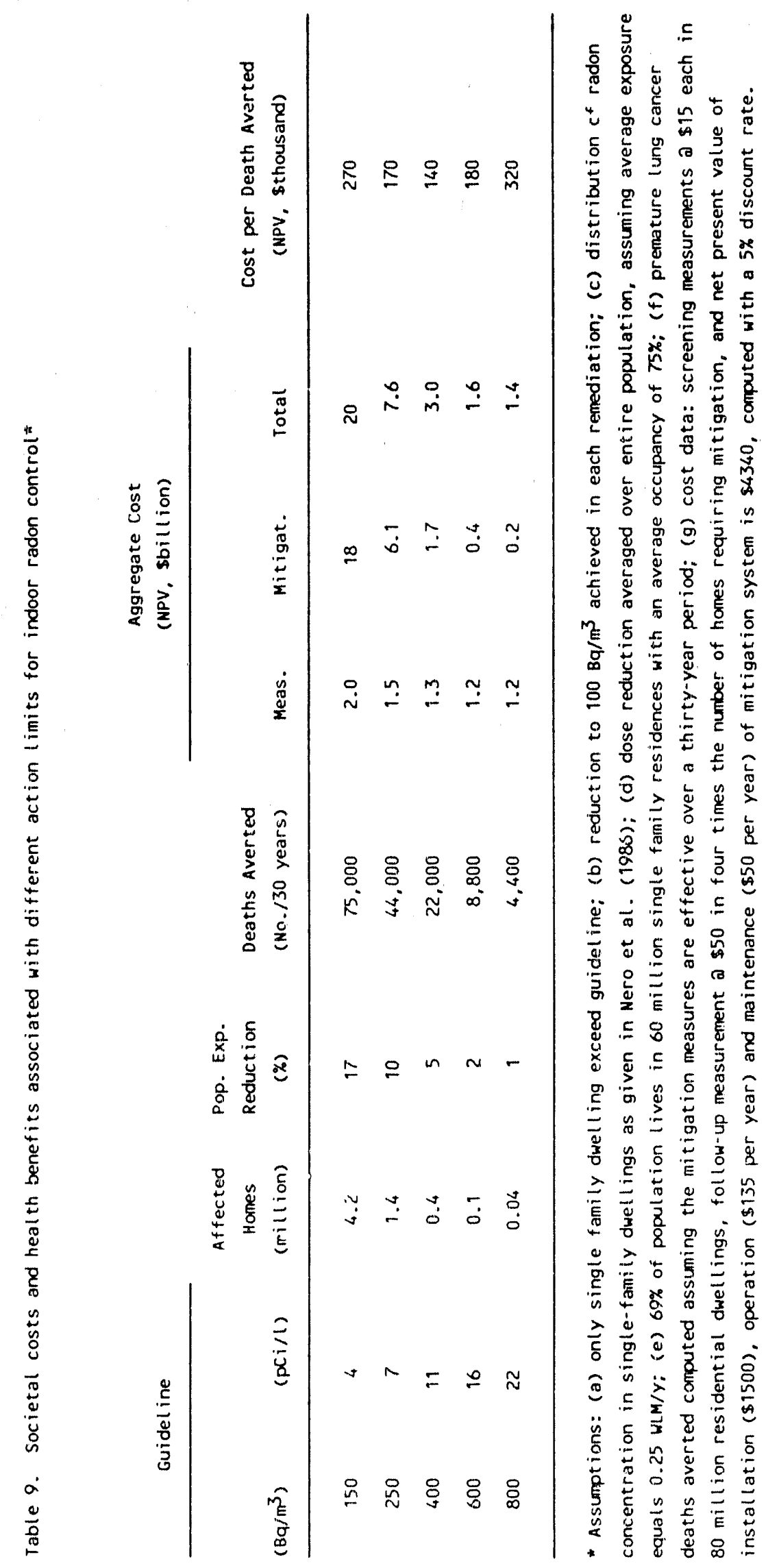


criterion. However, most of the cost would be associated with mitigation, and the cost of remediation per household may be higher. Furthermore, the average reduction in exposure per home remediated would be smaller. At present there is little practical experience with reducing indoor radon to il concentration below $150 \mathrm{~Bq} / \mathrm{m}^{3}$.

Because of the apparent synergistic interaction between smoking and radon (NAS 1988), and because of the mobility of the U.S. population, the cost of controlling indoor radon is less attractive to certain individuals than to society as a whole. Consider, for example, an owneroccupied residence inhabited by four lifelong nonsmokers: two adults, aged 40 ' male and female), and two children, aged 10 (male and female). Assume that the home is to be occupied for ten years before the family moves, that the average indoor radon concentration is $260 \mathrm{~Bq} / \mathrm{m}^{3}$ (the average concentration in households with a concentration above $150 \mathrm{~Bq} / \mathrm{m}^{3}$ according to the lognormal representation of the distribution from Nero et al. 1986), and that remediation at the average cost cited earlier would reduce the average indoor concentration to $100 \mathrm{~Bq} / \mathrm{m}^{3}$. The average net present value of the homeowner's cost for remediation would be approximately $\$ 3000^{8}$ The remediation would lead to a cumulative reduction in exposure per household of 33 WLM for the four occupants over ten years. ${ }^{9}$ From the risk projections of the BEIR IV commitiee, the lifetime risk of one lung cancer death in the family would be 0.0227 in the absence of remediation and 0.0216 with remediation. The informed homeowner would be faced with this question: Is it worthwhile to spend $\$ 3060$ tol'ay to reduce the risk by about $5 \%$ in relative terms (or about 1 in 1000 in absolute terms) that someone in my family will die of lung cancer? For a cohort of such households, an average of one lung cancer death would be avoided for every 900 homes remediated. Thus, the average cost (net present value) per lung cancer death averted is $\$ 2.7$ million, not including the expense of initially identifying the households. Since the costs of remediation re not strongly dependent on the initial radon concentration, applying control becomes progressively more attractive at higher indoor concentrations, but progressively less so at lower concentrations. Also, the initial expense of installing a radon-mitigation system may be recovered when the home is sold, thereby reducing the overall mitigation cost to the present homeowner.

From the perspective of a household in which there are smokers, the cost of radon remediation appears more attractive. If, in the previous example, all four family members were - or, in the case of the children, would become - smokers, the lifetime risk of at least one lung cancer death in the family would be 0.35 without remediation and would be reduced by 0.016 (to 0.33) with radon mitigation. The reduction in absolute risk is more than an order of magnitude larger than for the nonsmoking family at the same cost. On average, one lungcancer death would be averted in a cohcrt of 60 such households in which mitigation was applied, implying an average cost per lung cancer death averted of only $\$ 180,000$ (again excluding the cost of initially identifying the houses). However, in the absence of incentives such as low-interest loans ior remediation, it seems unlikely that most smokers would make the necessary investment to reduce the radon-related risk of lung mncer when the dominant cause of their risk is smoking.

From a public health perspective, the goal of reducing lung cancer incidence also may be more easily met by changing the population's smoking habits rather than by aggressive measures to reduce indoor radon concentrations. It is estimated that $85 \%$ of the annual lung cancer death rate of 130,000 cases in the United States is due to cigarette smoking (National Center for Health Statistics 1988; U.S. Department of Health and Human Services 1982). A permanent

${ }^{8}$ Installation cost of $\$ 1500$, plus $\$ 185$ per year in operation and maintenance expenses, ciiscounted at an annual rate of $5 \%$.

${ }^{9}$ Reduction by $160 \mathrm{~Bq} / \mathrm{m}^{3}$ corresponds to $0.84 \mathrm{WLM} \mathrm{y}^{-1}$ person $^{-1}$ in avoided exposure assuming $75 \%$ occupancy and $50 \%$ equilibrium between radon's decay products and radon. 
reduction by about $3 \%$ in the number of cigarette smokers would reduce the annual mortality due to lung cancer by the same amount as a radon-mitigation program that succeeded in achieving the EPA/CDC recommendations. The overall health benefit from reducing smoking to this extent would be much larger, since lung cancer is only one of several diseases to which smoking contributes. That is, a completely surcessful program, judged by the EPA/CDC guidance, would achieve a health benefit (occurring mostly among smokers) equivalent to a $1 \%$ reduction in smoking.

By legislation, a long-term national goal was recently established to make air within buildings in the United States "as free of radon as the ambient air outside of buildings" (U. S. Congress 1988). The legislation contains several provisions to promote movement towards this goal, such as a requirement that EPA develop model construction standards for new buildings.

The implications of measures needed to achieve this goal are staggering. The indoor radon concentration can only be reduced to the outdoor level by greatly reducing radon entry from soil, potable water, and building materials. As it is unlikely that radon ent:y from these sources could be completely prevented, it would also be necessary to remove radon from indoor air by filtration, for example with activated carbon (Bocanegra and Hopke 1989).

Whether this goal could be achieved in the existing housing stook is unclear. Certainly, substantial improyements in radon measurement and mitigation technology would be required. Even if it is technically feasible, the costs would be prohibitively large, on the order of $\$ 1$ trillion. ${ }^{10}$

If this long-term national goal were achieved, the average cumulative exposure of the U.S. population to radon decay products would be lowered by about $75 \%,{ }^{11}$ reducing the annual mortality from radon-related lung cancers by about 11,000 cases per year $(10,000$ cases averted per year among smokers, 900 among nonsmokers). An annual incidence of about 4,000 lung cancer deaths still would be attributed to radon exposure. Again assuming a 30 -year effectiveness of mitigation, the cost per lung cancer averted is estimated to be about $\$ 3$ million.

Because mitigation measures cost less to apply in new construction than in remediation, this :bjective would be less impractical, but still difficult and costly to achieve, if it were implemented gradually in new housing. Even in this case, though, an annual investment of at least several billion dollars might be required to compensate for an expense of several thousand dollars in each of a million new housing units.

${ }^{10}$ Only a rough estimate is possible, since there is no experience with reducing radon to this extent. The average net present value of the initial and operating cost per household remediated is estimated to be in the range $\$ 10,000-\$ 16,000$. Mitigation steps (and approximate average costs) are these: (a) reduce soil gas entry to meet current EPA guideline, $\$ 3-5 \mathrm{~K}$; (b) further reduce soil gas entry to near zero $(\$ 3-5 \mathrm{~K})$; (c) use activated charcoal or alternative filtration technique to remove radon from indoor air $(\$ 4-6 \mathrm{~K})$; (d) limit radon concentration in potable water supplies (small); and (e) limit radon from building materials (small). These measures would have to be applied in most of the 90 million U.S. residences to achieve the long-term national goal. If applied in 70 million households, the total net present value of the cost would be on the order of $\$ 1$ trillion.

${ }^{11}$ Continuous exposure to the average outdoor level of $9 \mathrm{~Bq} / \mathrm{m}^{3}$ results in an estimated cumulative exposure to radon decay products of $0.063 \mathrm{WLM}^{-1}$, or $25 \%$ of the estimated current exposure, 0.25 WLM $y^{-1}$. 


\section{Pitfalls and problems.}

For the analyses of the previous section it was assumed that current technology for radon measurement and mitigation would be applied ef fectively. In fact, a number of problems may prevent policy objectives from being achieved efficiently, as discussed in this section.

Most of the mitigation experience in the United States has been developed over the past few years in New York, New Jersey, Pennsylvania, and Washington. This experience is too recent to yield reliable information on the long-term efficacy of remedial measures, a necessary feature of an effective control program. Studies conducted a few years after installation indicate that a monitoring and maintenance program is necessary to ensure that mitigation measures continue to function effectively (Prill et al. 1989; Nitschke et al. 1988). Moreover, building practices vary widely across the country, so that the experience gained in one region may not be directly transferrable to another.

Quality assurance in radon detection is promoted by a measurement proficiency program established by the Environmental Protection Agency (Radon Technical Information Service 1988). This program is necessary; however, it is not clear that it is sufficient to ensure accurate measurements at concentrations near the guideline. In the test protocol, several detectors are exposed to an elevated, constant concentration of radon in a controlled atmosphere. By contrast, in measuring indoor concentrations, one or two detectors are exposed to a varying concentration of radon, which is often much lower than that used in the test protocol, in an atmosphere in which tenperature and humidity may be uncontrolled. Moreover, "blind" tests - i.e., those in which the vendors do not know that their detectors are being tested - are only now being incorporated into the program. As a result, the true measurement uncertainty for a single measurement in a residence may be larger than suggested by the $25 \%$ criterion for passing the test.

To achieve the long-term national goal, substantial improvements in measurement and mitigation technology would be required. Inexpensive monitoring devices routinely used for screening and longer-term integrated measurements are not sufficiently sensitive to measure outdoor radon concentrations accurately (a necessity for determining that the goal is being met). Equally important, there has been no direct experimental demonstration that indoor concentrations of radon can be practically reduced to outdoor levels.

Furthermore, under current practice, one can expect many houses to be misclassified with respect to the $150 \mathrm{~Bq} / \mathrm{m}^{3}(4 \mathrm{pCi} / \mathrm{l})$ guideline. The EPA protocol entails a two-day screening measurement, under closed-house conditions, on the lowest livable level of the home. The intention is to produce the highest indoor concentration that may be sustained in the home. The screening measurement permits rapid action for those homes in which the indoor radon concentration is exceedingly high. However, indoor radon concentrations fluctuate markedly, even under closed-house conditions, and the fluctuation periods can exceed the two-day screening measurement period (Scott 1989). One cannot conclude from a single screening measurement result below $150 \mathrm{~Bq} / \mathrm{m}^{3}$ that the annual average result for the household is below the guideline.

The results of screening measurements have also been misinterpreted in projecting the magnitude of the radon problem in U.S. housing (cf. Section II.A). Surveys have been conducted using the screening protocol in twenty-five states over the past few years. A significant fraction of the households in each state yielded measurements exceeding $150 \mathrm{~Bq} / \mathrm{m}^{3}$, a fact that was used to substantiate the recommendation for screening measurements in most homes. These results overstate the size of the problem because the measurements were made in closed houses and often in basements. Furthermore, due to temporal fluctuations, an 
overestimate of the fraction of homes exceeding $150 \mathrm{~Bq} / \mathrm{m}^{3}$ would also result from the twoday measurement protocol even if the measurements were conducted under ordinary living conditions. ${ }^{12}$

The objectives of current EPA/CDC policy cannot be met without widespread public compliance. The extent to which the U.S. public will follow these recommendations is not known. However, experience in New Jersey suggests that even with a highly publicized and large local radon problem, and despite substantial support and encouragement from governmental agencies, only a small fraction of residents have had a measurement made of the radon concentration in their home (Bynum et al. 1988). Furthermore, much of the measurement and mitigation effort has occurred with real estate transfers, suggesting that economic concerns about radon exceed personal health concerns.

To an extent, the decisions about whether to measure and to mitigate are appropriately left to individual residents. However, there is a public interest in reducing indoor radon exposures. Because the U.S. population is highly mobile, a homeowner will be less likely to adopt control measures than would the society operating as a unit unless mitigation is required or unless its cost is recovered through increased property values. Furthermore, the costs of medical care are largely borne by society through insurance, rather than directly by individuals. In addition, under the current policy, remediation is unlikely in homes that are rented.

Although the projected health consequences associated with indoor radon exposure are large, it has not yet been possible, and will be difficult in the future, to demonstrate a compelling association between environmental radon exposure and lung cancer rates. Also, because of the separation in time between exposure and effect, and because of the large incidence of lung cancer from smoking, a specific lung cancer death cannot reliably be attributed to radon exposure. Amidst this uncertainty, arguments are put forth that indoor exposures to radon are much less hazardous than the risk projections presented in this paper would suggest (Cohen $1989 \mathrm{~b})$. Such controversy diminishes the will of the public to take corrective action.

In addition, it is important to recognize that even with an aggressive program to reduce radon exposures, benefits will not be observed quickly. There is a latency period following exposure before the onset of illness. Thus, following a change in the population exposure, no change in lung cancer rate would be expected until the latency period (five years, according to the BEIR IV committee (NAS 1988)) has elapsed. Evidence also suggests that increased risk due to exposure persists (with some reduction in magnitude) throughout life. Therefore, decades would elapse following full implementation of remediation measures before a substantial fraction of the benefit of reduced risks would be expressed, and even then the reduced risks may not be detectable, particularly if cigarette smoking habits continue to change.

According to the parameters of the lognormal distribution of indoor radon concentrations presented in Nero et al. (1986), there are approximately 70,000 homes in the United States in which the average radon concentration exceeds $800 \mathrm{~Bq} / \mathrm{m}^{3}$. At this level, a $75 \%$-time occupant receives a cumulative exposure of $4 \mathrm{WLM}^{-1}$, equal to the limit for underground uranium miners (Federal Register 1968). For an individual exposed at this level throughout life, the

\footnotetext{
${ }^{\Gamma 2}$ Because of the large temporal fluctuations in concentrations, some homes with annual averages below $150 \mathrm{~Bq} / \mathrm{m}^{3}$ will be measured at greater than $150 \mathrm{~Bq} / \mathrm{m}^{3}$, and, conversely, some homes with annual averages above $150 \mathrm{~Bq} / \mathrm{m}^{3}$ will be measured at concentrations below this guideline. But, because of the shape of the distribution of indoor concentrations, among all homes with mean concentrations within an increment, $\epsilon$, of the $150 \mathrm{~Bq} / \mathrm{m}^{3}$ guideline, many more are below rather than above this level. Therefore, the incidence of false positives is expected to exceed the incidence of false negatives, and so the number of homes above the guideline is overestimated.
} 
risk of lung cancer is extraordinarily high, ranging from $2 \%$ for female nonsmokers to $33 \%$ for male smokers (NAS 1988). ${ }^{13}$ By almost any criterion, this level of health risk due to unsuspected exposure to radon's decay products in one's home is unacceptable. It should be a high priority to rapidly identify these homes and take corrective measures to reduce exposures in them. The establishment of the stringent long-term national goal may divert attention and resources from those homes in which the problem is acute. Indeed, strict emphasis on achieving the EPA guideline in the millions of homes that have concentrations only moderately above $150 \mathrm{~Bq} / \mathrm{m}^{3}$ may dilute the overall effectiveness of the effort to identify and take remedial action in the small fraction of these homes in which the indoor radon concentration is extraordinarily large.

\section{Moving towards a coherent policy.}

In this section, some important chal acteristics of a reasoned response to indoor radon are discussed. These issues are, at best, underemphasized in the current approach. In our view, these aspects must be explicitly incorporated to make effective progress toward the goal of reducing indoor radon exposures.

As discussed in Section VI.A, a comprehensive control straiegy for limiting exposure to indoor radon may be viewed as having three key elements: a system of exposure guidelines, mcthods for classifying buildings with respect to the guidelines, and a framework for prescribing and implementing control techniques. Each of those elements can be found at a minimal level in present federal policy. However, formulating these elements independently does not, by itself, constitute the development of a comprehensive strategy. Effort must also be devoted to resolving some fundamental, underlying issues in controlling radon and other indoor air pollutants as well. For example, the respective responsibilities of the concerned parties owners, occupants, builders, and local, state, and federal governments - remain ambiguous. A cohesive strategy for controlling indoor radon cannot be developed without integrating technical assessments with an understanding of political, economic, and legal aspects of the issue. Beyond establishing objectives, methods for achieving these goals must be carefully planned. To do so requires a concerted effort that is more explicit and detailed than has so far been undertaken.

Turning to more specific issues, the EPA/CDC recommendation that a screening measurement be made in virtually every home is an inefficient means to achieve the goal of identifying homes with annual average concentrations exceeding $150 \mathrm{~Bq} / \mathrm{m}^{3}(4 \mathrm{pCi} / \mathrm{l})$. The major value of a screening measurement is to identify homes in which the radon concentrations are so high that remedial action should be taken before an annual or seasonal average measurement can be completed. (Even in homes with concentrations above $200 \mathrm{pCi} / 1\left(7400 \mathrm{~Bq} / \mathrm{m}^{3}\right)$, it is recommended that a follow-up short-term (one-week) measurement be made before undertaking remedial measures.) As discussed in Sections II.C and VI.A.3, these homes constitute a very small fraction of the housing stock, and, because they occur in clusters, can be identified more efficiently by methods other than measuring every household. Increased attention should be given to the possibility of using geological information, in combination with radon concentration measurements in a sample of homes in a region, to identify areas in which a significant probability of very high concentrations exists (Duval 1983; Kothari and Han 1984; Revzan et al. 1988). Greater effort should also be devoted to developing mechanisms for providing information to residents on the distribution of indoor radon concentrations in their communities so they can make informed decisions on wether to measure radon in their homes (Bynum et al. 1988).

${ }^{13}$ By comparison, in the absence of radon exposure, the lifetime risk of lung-cancer is estimated to be $0.6 \%$ for female nonsmokers and $12 \%$ for male smokers. 
Because of the large, naturally occurring, temporal variations in indoor radon concentrations, screening measurements cannot be considered a reliable indicator of long-term average exposure conditions, the parameter of concern for assessing the risk of lung cancer. Misinterpretation of the results of screening measurements has generated much confusion about the extent of the indoor radon problem. Instead of encouraging screening measurements, measurements of long-term average concentrations under actual living conditions should be promoted. The results of these measurements provide a superior basis for making decisions about remediation.

To a greater extent than for the control of many other environmental pollutants, public support and cooperation are vital for achieving reductions in radon exposure. Public information and training provided by the EPA contribute to this goal, but more could be done. For example, EPA's establishment of a remedial action guideline may yield a de facto standard that affects much of the population without any formal mechanism for criteria development, scientific review, or public comment. The federal government's response to indoor radon should be reassessed through a means that encourages discussion and debate.

Turning to consideration of the TSCA amendment, much of the cost to achieve the long-term national goal as presently stated would be spent to achieve small marginal reductions in exposure. Most of the benefit of this goal could be realized at a small fraction of the cost by establishing a target concentration in the vicinity of $25-50 \mathrm{~Bq} / \mathrm{m}^{3}$ and by attempting to achieve this goal only in new construction. The long-term goal should be supported by a plan for attainment, including a time frame.

In general, wherever possible, federal policy should promote a steady development of radon measurement and mitigation expertise, rather than a boom-bust cycle. Indoor radon is a widespread problem and there is a long history of human exposures. A crash program to identify and mitigate homes above the present guideline is inefficient. If a backlash of public opinion were to result from, for example, an overstatement of the magnitude of the problem, then the ultimate benefit may be smaller than that which could be achieved by a more efficient, gradual response. Steady development would permit the growth of an infrastructure of trained individuals and businesses. Mitigation measures could be steadily improved. Better measurement techniques might be developed. More information would become available on the health risks of environmental exposures, yielding a stronger basis for mitigation decisions. These henefits cannot be realized if the radon problem is treated as an epidemic that requires rapid countermeasures with little regard for scientific uncertainties and accompanying costs.

A steady response will be encouraged if our primary short-term goal is to identify and apply remedial measures in houses having indoor concentrations that are extremely high. By reducing radon concentrations in these homes, we can ensure that individual risks due to indoor radon exposure are not extraordinarily high relative to those that are routinely accepted in association with other activities, such as riding in a car. With vigorous and focused effort, it might be possible to identify and correct excessive concentrations in these homes within a few years. Ultimately, any substantial reduction in the cumulative population exposure would require concentration reductions in a large fraction of the building stock. If this goal is to be sought, it could best be accomplished gradually, with relatively stringent controls applied to new construction and, possibly, a second level of control in existing buildings. As decades would be required to achieve such a goal, the opportunity is available to deliberately consider its merits and costs, and to improve the basis for making decisions by developing additiona: information. We are most likely to reduce radon-related health risks at reasonable cost only if we apply a sustained and steady effort to the froblem of reducing indoor exposures. 


\section{Acknowledgments}

This work was supported by the Director, Office of Enetgy Research, Office of Health and Environmental Research, Human Health Assessments Division and Pollutant Characterization and Saf ty Research Division of the U.S. Department of Energy under Contract No. DE-AC03$76 \mathrm{SF} 00$ ' 98.

We would like to thank R.C. Diamond, C.V. Gogolak, E.O. Knutson, W.M. Lowder, R.G. Sextro, and H.A. Wollenberg, Jr., as well as others in and for the U.S. Department of Energy, for their comments on this report. We are also grateful to T.D. Gartner and R.M. Rodriguez for their efforts in preparinz the manuscript. 


\section{REFERENCES}

AECB 1978-80: Atomic Energy Control Board, Canada, 1st, 2nd, and 3rd Workshops on radon and radon daughters in urban communities associated with uranium mining and processing, Elliot Lake, Ontario, March 7-9, 1978, Bancroft, Ontario, March 13-14, 1979, and Port Hope, Ontario, March 13-14, 1980.

Aitchison, J. and Brown, J.A.C. (1957). The Lognormal Distribution, Cambridge Univ. Press, Cambridge, England.

Akerblom, G., Andersson, P. and Clavensjo, B. (1984). Soil gali radon -- a source for indoor radon daughters, Kad. Prot. Dos. 7, 49.

Alter, H.W. and Fleischer, R.L. (1981). Passive integrating radon monitor for environmentai monitoring, Health Phys., 40, 693.

Alter, H.W. and Oswald, R.A. (1987). Nationwide distribution of indoor radon concentrations: a preliminary data base, J. Air Pollution Control Ass. 37, 227.

Alter, H.W. and Oswald, R.A. (1989). Nationwide distribution of indoor radon measurements, in USEPA 1989 b, p. 3-1.

Aoyama, T. et al. (1987). Long-term measurements of radon concentrations in the living environments in Japan: a preliminary report, in Hopke 1987, p. 124.

APCA (1986). Indoor Radon, Air Pollution Cont. Assoc. Specialty Conf. Proceedings SP-54, Pittsburgh, Pa.

APCA (1987). Indoor Radon II, Air Pollution Cont. Assoc. Specialty Conf. Proceedings SP-60 and Supplement, Pittsburgh, Pa.

ASHRAE (1989). Ventilation for acceptable indoor air quality, Standard 62-1989, American Society of Heating, Refrigeration, and Air-Conditioning Engineers, Atlanta, GA.

Arvela, H., Voutilainen, A., Makelainen, I., Castren, O. and Winqvist, K. (1988), Comparison of predicted and measured variations of indoor radon concentration, Rad. Prot. Dos. 24, 231.

Asikainen, M. and Kahlos, H. (1980). Natural radioactivity of drinking water in Finland, Health Phys. 39, 77.

Bigu, J. (1985). Radon daughter and thoron daughter deposition velocity and unatached fraction under laboratory-controlled conditions and in underground uranium mines, J. Aerosol Sci., 16, 157.

Bocanegra, R. and Hopke, P.K. (1989). Theoretical evaluation of indoor radon control using a charcoal adsorption system, J. Air Pollution Control. Assoc. 39, 305.

Borak, T.B., Woodruff, B., and Toohey, R.E. (1989). A survey of winter, summer, and annual average ${ }^{222} \mathrm{Rn}$ concentrations in family dwellings, Health Phys. 57, 465. 
BPA (1986). Radon monitoring results from BPA's residential weatherization Program, Bonneville Power Administration Report DOE/BP-71.

Browne, E. and Firestone, R.B. (1986). Table of radioactive isotopes, (V.S. Shirley, ed.), Wiley: New York.

Bruno, R.C. (1983). Sources of indoor radon in houses: a review, J. Air Pollution Control Assoc. 33, 105.

Bruno, R.C. (1983b). Verifying a model of radon decay product behavior indoors, Health Phys., 45,471 .

Budnitz, R.J. (1974). Radon-222 and its daughters - a review of instrumentation for occupational and environmental monitoring, Health Phys., 26, 145.

Bynum, J., Klotz, J., Cahiil, M., and Nicholls, G. (1988). The rationale and experiences in implementing New Jersey's Radon Program, paper 88-106.5, Air Pollution Control Association, Pittsburgh, PA.

California Department of Health Services (1989). Radon in California schools, news release, Sacramento, CA, April 20.

Castren, O., Voutilainen, A., Winqvist, K., and Makelainen, I. (1985). Studies of high indoor radon areas in Finland, Sci. Total Environ., 45, 311.

Castren, O., Makelainen, I., Winqvist, K., and Voutilainen, A. (1987). Indoor radon measurements in Finland: A status report, in Hopke 1987, p. 97.

CDM, Camp Dresser \& MeKee (1989). Statewide Scientific Study of Radon, Summary Report, New Jersey Department of Environmental Protection, Trenton, NJ.

Chu, K.--D. and Hopke, P.K. (1988). Neutralization kinetics for polonium-218, Environ. Sci. Technol., 22, 711.

Cliff, K.D. (1978). Assessment of airtorne radon daughter concentrations in dwellings in Great Britain, Phys. Med. Biol., 23, 696.

Cliff, K.D., Wrixon, A.D., Miles, J.C.H., and Lomas, P.R. (1987). Remedial measures to reduce radon concentrations in a house with high radon levels, in Hopke 1987, p. 536.

Cohen, B.L. (1986). A national survey of ${ }^{222} \mathrm{Rn}$ in U.S. homes and correlating factors, Health Phys. 51, 175.

Cohen, B.L. (1987). Radon - A homeowner's guide to detection and control, Consumers Union, Mount Vernon, NY.

Cohen, B.L. (1987b). Personal communication and draft paper.

Cohen, B.L. (1989). Table of "Radon levels by states and counties."

Cohen, B.L. (1989b). Correlation between mean radon levels and lung cancar rates in U.S.

counties: A test of the linear-no threshold theory, in IJSEPA 1989b, p. 2-15. 
Cohen, B.L. and Cohen, E.S. (1983). Theory and practice of radon monitoring with charcoal adsorption, Health Phys., 45, 501.

Cohen, B.L. and Nason, R. (1986). A diffusion barrier charcoal adsorption collection for measuring $\mathrm{Rn}$ concentrations in indoor air, Health Phys. 50, 457.

Cohen. B.L., Kulwicki, D.R., Warner, K.R., Jr., and Grassi, C.L. (1984). Radon concentrations inside public and commercial buildings in the Pittsburg area, Health Phys., 47, 399.

Costle, D.M. (1979). Recommendations for radiation protection of persons residing on phosphate lands, contained in a letter from U.S. EPA Administrator Costle to the Covernor of Florida, 30 May.

Culot, M.V.J., Schiager, K.J., and Olson, H.G. (1978). Development of a radon barrier, Health Phys, 35, 375.

Dietz, R.N., and Cote, E.A. (1982). Air infiltration measurements in a home using a convenient perfluorocarbon tracer technique, Environ. Int., 8, 419.

Doyle, S.M., Nazaroff, W.W., and Nero, A.V. (1984). Time-averaged indoor radon concentrations and infiltration rates sampled in four U.S. cities, Health Phys., 47, 579.

DSMA Atcon Ltd. (1983). Review of existing instrumentation and evaluation of possibilities for research and development of instrumentation to determine future levels of radon at a proposed buildings site, Atomic Energy Control Board Report INFO-0096, Ottawa, Canada.

Duval, J.S. (1983). Composite color images of aerial gamma-ray spectrometric data, Geophys. 48, 722.

Duval, J.S. (1989). Radon prediction using gamma-ray spectrometric data (abstract), EOS 70, 496.

Duval, J.S., Otton, J.K., and Jones, W.J. (1989). Estimation of radon potential in the Pacific Northwest using geological data, Bonneville Power Administration Report DOE/BP-1234.

Ericson, S.-O. (1987). Modified design in new construction prevents infiltration of soil gas that carries radon, in Hopke 1987, p. 526.

Federal Register (1968). Vol. 33, 19947, 28 December

Federal Register (1986). Vol. 51, 34836, 30 September

Fisenne, I.M., George, A., and McGahan, M. (1983). Radon measurement intercomparisons, Health Phys. 45, 553.

Fisk, W.J. and Turiel, I. (1983). Residential air-to-air heat exchangers: Performance, energy-savings, and economics, Energy and Buildings, 5, 197.

Fisk, W.J., Spencer, R.K., Grimsrud, D.T., Offerman, F.J., Pedersen, B., and Sextro, R.G. (1984), Indoor air quality control techniques: a critical review, Lawrence Berkeley Laboratory Report, LBL-16493. 
Fisk, W.J., Flexser, S., Gadgil, A.J., Holman, H.-Y., Modera, M.P., et al. (1989). Monitoring and modeling for radon entry into basements: a status report for the small structures project, Lawrence Berkeley Laboratory Report, LBL-27692, Berkeley, CA.

Garbesi, K. and Sextro, R.G. (1989). Modeling and field evidence of pressure-driven entry of soil gas into a house inrough permeable below-grade walls, Environ. Sci. Technol. 23, 1481.

George, A.C. (1972). Indoor and outdoor measurements of natural radon and radon decay products in New York City air, in Natural Radiation Environment, Adams, J.A.S., Lowder, W.M., and Gesell, T.F., Eds., CONF-720805, National Technical Information Services, Springfield, VA, p. 741.

George, A.C. (1980), Radon and radon daughter field measurements, in Proc. Seminar on Traceability for Ionizing Radiation Measurements, National Bureau of Standards, Washington, DC.

George, A.C., and Breslin, A.J. (1980). The distribution of ambient radon and radon daughters in residential buildings in the New York-New Jersey area, in Natural Radiation Environment III, Gesell, T.F., and Lowder, W.M., Eds., Tech. Info. Ctr/DOE CONF-780422, Springfield, VA, p. 1272.

George, A.C., Knutson, E.O., and Tu, K.W. (1983). R.adon daughter plateout - I. Measurements, Health Phys., 45, 439.

George, A.C., Duncan, M., and Franklin, H. (1984). Measurements of radon in residential buildings in Maryland and Pennslyvania, U.S.A., Radiat. Prot. Dosim. 7, 291.

Gesell, T.F. (1973). Some radiological aspects of radon-222 in liquified petroleum gas, in Nobel Gases, Stanley, R.E. and Moghissi, A.A., Eds., U.S. Energy Research \& Dev. Admin.

CONF-730915, Las Vegas, NV, p. 612.

Gesell, T.F. (1983). Background atmospheric ${ }^{222} \mathrm{Rn}$ concentrations outdoors and indoors: A review, Health Phys., 45, 289.

Girman, J.R., Apte, M.R., Traynor, G.W., Allen J.R., and Hollowell, C.D. (198`)), Pollutant emission rates from indoor combustion appliances and sidestream cigarette smoke, Environment International 8, 213.

Gobel, R., and Socolow, R. (1988). High radon houses: implications for epidemiology and risk assessment, draft manuscript (Sept. 30).

Granlund, C.L. (1988). Personal communication

Green, B.M.R., Brown, L., Cliff, K.D., Driscoll, C.M.H., Miles, J.C., and Wrixon, A.D. (1985). Surveys of natural radiation exposure in UK dwellings with passive and active measurement techniques, Sci. Total Environ., 45, 459.

Grimsrud, D.T. (1988). Personal communication.

Grimsrud, D.T., Modera, M.P., and Sherman, M.H. (1982). A predictive air infiltration model -- long-term field test validation, ASHRAE Trans. 88, 1351. 
Grot, R.A. and Clark, R.E. (1981). Air leakage characteristics and weatherization techniques for low'-income housing, in Thermal Performance of the Exterior Envelopes of Buildings, Report ASHRAE SP28, American Society of Heating, Refrigerating and Air Conditioning Engineers, New York, p. 178 .

Gunning, C. and Scott, A.G. (1982). Radon and thoron daughters in housing. Health Phys., 42, 527.

Haghighat, F., Chandrashekar, M. and Unny T.E. (1987). Thermal behaviour of buildings under random conditions, Applied Mathematical Modeling, Vol. 11, p. 349

Haghighat, F., Fazio, P, and Unny, T.E. (1988). A predictive stochastic model for indoor air quality, Building and Environment, 23, 195.

Harley, N.H. (1984). Comparing radon daughter dose: environmental versus underground exposure, Rad. Prot. Dos., 7, 371.

Hartwell, T,D., Perritt, R.L., Sheldon, L.S., Cox, B.G., Smith, M.L., and Rizutto, J.E. (1987). Distribution of radon levels in New York State homes, in Seifert et al. 1987, Vol. 2, p. 398; for more recent results, cf. R.L. Perritt et al., Distribution of radon levels in New York State homes, in USEPA 1989b, p. 3-37, and R.L. Perritt et al., manuscript submitted to Health Phys. 1989.

Henschel, D.B. and Scott, A.G. (1986). The EPA program to demonstrate mitigation measures for indoor radon: initial results, in APCA 1986, p. 110.

Hess, C.T., Weiffenbach, C.V., and Norton, S.A. (1983). Environmental radon and cancer correlations in Maine, Health Phys., 45, 339.

Hess, C.T., R.L. Fleischer, L.G. Turner (1985). Field and laboratory tests of etched track detectors for ${ }^{222} \mathrm{Rn}$ : summer-vs-winter variations and tightness effects in marine houses, Health Phys. 49, 65.

Hildingson, O. (1982). Radon measurements in 12,000 Swedish homes, Environ. Int., 8, 67.

Holub, R.F., Droullard, R.F., Borak, T.B., Inkret, W.C., Morse, J.G., and Baxter, J.F. (1985), Radon-222 and ${ }^{222} \mathrm{Rn}$ progeny concentrations measured in an energy-efficient house equipped with heat exchanger, Health Phys., 49, 267; see also related correspondence (1988) in Health Phys. 55, $1005 \mathrm{ff}$.

Hopke, P.K. (Ed.) (1987). Radon and its decay products - occurrence, properties and health effects, American Chemical Soc. Symposium Series 331, Washington, D.C.

Hopke, P.K. (1988). The initial atmospheric behavior of radon decay products, Report DOE/ER-0375, U.S. Department of Energy, Washington, D.C.

Horton, T.R. (1985). Nationwide occurrence of radon and other natural radioactivity in public water supplies, Report EPA 520/5-85-008, U.S. Environmental Protection Agency, Eastern Environmental Radiation Facility, Montgomery, AL. 
Hubbard, L. Gadsby, K., Buhac, D., Lovell, A., Harrje, D., Socolow, R., Matthews, T., Dudney, C., and Sanchez, D. (1988), Radon entry into detached dwellings: house dynamics and mitigation techniques, Rad. Prot. Dos. 24, 491.

Hubbard, L.M, Bolker, B., Sincolow, R.H., Dickerhoff, D., and Mosley, R.B. (1989), Radon dynamics in a house heated a ternatively by forced air and by electric resistance, in USEPA 1989 b, p. 6-1.

ICRP (1984). Principles for Limiting Exposure of the Public to Natural Sources of Radiation, International Commission on Radiological Protection, publication 39, Annals of the ICRP, 14, No. 1 .

Ingersoll, J.G. (1983). A survey of radionuclide contents and radon emanation rates in building materials used in the U.S.. Health Phys., 45, 363.

Jacobi, W. (1972). Activity and potential alpha energy of radon 222 and radon 220 daughters in different air atmospheres, Health Phys., 22, 441.

James, A.C. (1988). Lung dosimetry, in Nazaroff and Nero 1988, p. 259.

James, A.C. (1989a). Reassessment of factors influencing lung dose from radon daughters, paper presented at a technical exchange meeting on "Assessing Indoor Radon Health Risks," Grand Junction, Sept. 18-19.

James, A.C. (1989'). Personal communication.

Johnson, R.H., Bernhardt, D.E., Nelson, N.S., and Galley, H.W. (1973). Radiological health significance of radon, in Noble Gases, Stanley, R.E. and Moghissi, A.A., Eds., U.S. Energy Research \& Dev. Admin. rep CONF-730915, Las Vegas, NV, p. 532.

Jonassen, N. (1984), Removal of radon daughters by filtration and electric fields, Rad. Prot. Dos., $7,407$.

Jonassen, N. and McLaughlin, J.P. (1988). Removal of radon and radon progeny from indoor air, in Nazaroff and Nero 1938, p. 435.

Keller, G., and Folkerts, K.H. (1984). A study on indoor radon, in Indoor Air: Radon, Passive Smoking, Particulates and Housing Epidemiology, Vol. 2, Berglund, B., Lindvall, T., and Sundell, J., Eds., Swedish Council for Building Research, Stockholm, p. 149.

Keller, G., Folkerts, K.H., and Muth, H. (1982). Activity concentrations of ${ }^{222} \mathrm{Rn},{ }^{220} \mathrm{Rn}$, and their decay products in German dwellings, dose calculations and estimate of risk, Radiat.

Environ. Biophys., 7, 263, cited in Schery 1986.

Kliest, J., Fast, T., Boley, J.S.M., van de Wiel, H., and Bloemen, H. (1989). The relationship between soil contaminated with volatile organic compounds and indoor air pollution, Environment International 15, 419.

Knutson, E.O. (1988). Modeling indoor concentrations of radon's decay products, in Nazaroff and Nero 1988, p. 161. 
Kothari, B.K, and Han, Y. (1984). Association of indoor radon concentrations with airborne surveys of uranium in surficial material, Northeastern Environ. Sci. 3, 30.

Kunz, C., Laymon, C.A., and Parker, C. (1988). Gravelly soils and indoor radon, in USEPA 1989 b, p. $5-75$.

Kunz, C.O. (1988). Indoor radon: source characterization, Environmental Progress, 7, 236.

Létourneau, E.G. (1986). Radon standard for Canada, in Indoor Air Quality in Cold Climates:

Hazards and Abatement Measures, D.S. Walkinshaw, ed., Air Pollution Control Association, Pittsburgh, PA. p. 488-495.

Lecierer, C.M. and Shirley, V.S. (1978). Table of Isotopes, 7th Ed., Wiley-Interscience, New York.

Liddament, M. and Allen, C. (1983). The validation and comparison of mathematical models of air infiltration, AIC 11, Technical Note, Air Infiltration Centre, Bracknell, Great Britain.

Los Angeles Times (1988). Results of radon survey

Loureiro, C. de Oliveira (1987). Simulation of the steady-state transport of radon from soil into houses with basements under constant negative pressure, LBL-24378.

Lubin, J.H. and Boice, Jr., J.D. (1989). Estimating Rn-induced lung cancer in the United States, Health Phys., 57, 417.

Maher, E.F., Rudnick, S.N. and Moeller, D.W. (1987). Effective removal of airborne ${ }^{222} \mathrm{Rn}$ decsy products in builcings, Health Phys. 53, 351.

Mansfield, R.H., III (1986). How radon gas will affect the relocation industry, Mfobility, July-A ugust, p. 6.

Martz, D.E., Harris, R.T., and Langner, G.H., Jr. (1989). The half-life of ${ }^{218} \mathrm{Po}$, Health Phys., 57,121 .

Martz, D.E., George, J.L., Mamich, S.T., and Langner, G.H., Jr. (1989b). Validation of the diffusion barrier charcoal cannister method, U.S. DOE Grand Juncticn Projects Office, report UNC/GJ-47 (TMC), Grand Junction, CO.

Mattson, M.E., Pollack, E.S., Cullen, J.W. (1987). What are the odds that smoking will kill you?, Amer. J. Public Health 77, 425.

Matthews, T.G., Wilson, D.L. TerKonda, P.K. Saultz, R.J., Goolsby, G., Burns, S.E., and Haas, J.W. (1989). Radon diagnostics: subslab communication and permeabiliity measurements, in USEPA 1989b, p. 6-45.

McCann, J., Horn, L., Girman, J.R., and Nero, A.V. (1987). Potential risks from exposure to organic carcinogens in indoor air, in S.S. Sanbhu et al. (Eds.) Short-Term Bioassays in the Analy'sis of Complex Environmental Mixtures $V$, Plenum, New York.

McGregor, R.G., Vasudev, P., Letourneau, E.G., McCullough, R.S., Pranil, F.A., and Taniguchi, H. (1980). Background concentrations of radon and radon daughters in Canadian homes, Health Phys., 39, 285. 
McLaughlin, J.P. and Wasiolek, P. (1988). Radon levels in Irish dwelling, Rad. Prot. Dos. 24, 383.

Mercer, T.T. (1976). The effect of particle size on the escape of recoiling RaB atoms from particulate surfaces, Health Phys., 31, 173.

Miksch, R.R., Hollowell, C.D., and Schmidt, H.E. (1982). Trace organic chemical contaminants in office spaces, Environment International 8, 129.

Moed, B.A., Nazaroff,W.W., Nero, A.V., Schwehr, M.B., and Van Heuvelen, A. (1985).

Identifying areas with potential for high indoor radon levels: analysis of the national airborne radiometric reconnaissance data for California and the Pacific Northwest, LBL- 16955.

Mowris, R.J. and Fisk, W.J. (1988). Modeling the effects of exhaust ventilation on ${ }^{222} \mathrm{Rn}$ entry rates and indoor ${ }^{222} \mathrm{Rn}$ concentrations, Health Phys. 54, 491.

Narasimhan, T.N., Tsang, Y.W., and Holman, H.-Y. (1989). On the potential of transient flow in advective radon entry into buildings, to be published in Geophys. Res. Letts.

NAS (1981). Indoor Pollutants, National Research Council, National Academy Press, Washington, D.C.

NAS (1988). Health Risks of Radon and Other Internally Deposited Alpha Emitters: Beir IV, Committee on the Biological Effects of Ionizing Radiations, National Research Council, National Academy Press, Washington, D.C.

National Center for Health Statistics (1988). Vital Statistics of the United States, 1986, DHHS Pub. No. (PHS) 88-1114, U.S. Government Printing Office, Washington, D.C., Table 8.5.

Nazaroff, W.W. (1988), Measurement techniques, in Nazaroff and Nero 1988, p. 491.

Nazaroff, W.W. and Cass, G.R. (1989a). Mass-transport aspects of pollutant removal at indoor surfaces, Environ. Int., in press.

Nazaroff, W.W. and Cass, G.R. (1989b). Mathematical modeling of indoor aerosol dynamics, Environ. Sci. Technol., 23, 157.

Nazaroff W.W. and Doyle, S.M. (1985). Radon entry into houses having a crawl space, Health Phys., 48, 265.

Nazaroff, W.W. and Nero, A.V. (1984). Transport of radon from soil into residences, in Indoor Air: Radon. Passive Smoking, Particulates and Housing Epidemiology, Vol. 2, Berglund, B., Lindvall, T., and Sundell, J., Eds., Swedish Council for Building Research, Stockholm, p. 15.

Nazaroff, W.W. and Nero, A.V. (Eds.) (1988). Radon and Its Decay Products in Indoor Air, Wiley, New York.

Nazaroff, W.W. and Sextro, R.G. (1989). Technique for measuring the indoor ${ }^{222}$ Rn source potential of soil, Environmental Science \& Technology, 23, 451.

Nazaroff, W.W., Boegel, M.L., Hollowell, C.D., and Roseme, G.D. (1981). The use of mechanical ventilation with heat recovery for controlling radon and radon-daughter concentrations in houses. Almos. Environ. 15, 263. 
Nazaroff, W.W., Boege!, M.L., and Nero, A.V. (1983). Measuring radon source magnitude in residential buildings, in Proc. International Meeting on Radon-Radon Progeny Measurements, Report 520/5-83/021, U.S. Environmental Protection Agency, Washington, DC, p. 101.

Nazaroff, W.W., Offerman, F.J., and Robb. A.W. (1983). Automated system for measuring air-exchange rate and radon concentration in houses, Health Phys., 45, 525.

Nazaroff, W.W., Feustel, H., Nero, A.V., Revzan, K.L., Grimsrud, D.T., Essling, M.A., and Toohey, R.E. (1985). Radon transport into a detached one-story house with a basement, Atmos. Env., 19, 31 .

Nazaroff, W.W., Lewis, S.R., Doyle, S.M., Moed, B.A., and Nero, A.V. (1987). Experiments on pollutant transport from soil into residential basements by pressure-driven air flow, Environ.

Sci. and Tech., 21459.

Nazaroff, W.W., Doyle, S.M., Nero, A.V., and Sextro, R.G. (1987b). Potable water as a source of airborne ${ }^{222} \mathrm{Rn}$ in U.S. dwellings: a review and assessment, Health Physics, 52, 281.

Nazaroff, W.W., Moed, B.A., and Sextro, R.G. (1988), Soil as a source of indoor radon: generation, migration, and entry, in Nazaroff and Nero 1988, p. 57.

NCRP (1984). Exposures from the Uranium Series with Emphasis on Radon and Its Daughters, National Council on Radiation Protection and Measiuements, Report No. 77, Bethesda, MD.

NCRP (1984b). Evaluation of Occupational and Environmental Exposures to Radon and Radin Daughters in the United States, National Council on Radiation Protection and Measurements, Report No. 78, Bethesda, MD.

NCRP (1985). A Handbook of Radioactivity Measurement Procedures, 2nd Ed., National Coucil on Radiation Protection and Measurements, Report No. 58, Bethesda, MD.

NCRP (1988). Measurement of Radon and Radon Daughters in Air. National Council on Radiation Protection Measurements, Report No. 97, Bethesda, MD.

NCRP (1989). Control of Radon in Houses, National Council on Radiation Protection and Measurements, Report No. 103, Bethesda, MD.

Nero, A.V. (1983). Airborne radionuclides and radiation in buildings: A review. Health Phys., $45,303$.

Nero, A.V. (1988). Controlling indoor air pollution, Scientific American, 258, 42.

Nero, A.V. (1988b). Elements of a strategy for control of indoor radon, in Nazaroff and Nero 1988, p. 459.

Nero, A.V. and Nazaroff, W.W. (1984). Characterising the source of radon indoors, Rad. Prot. Dos., 7, 23.

Nero, A.V., Berk, J.V., Boegel, M.L., Hollowell, C.D., Ingersoll, J.R., and Nazaroff, W.W. (1983). Radon concentrations and infiltration rates measured in conventional and energy-efficient houses, Health Phys., 45, 401. 
Nero, A.V., Schwehr, M.B., Nazaroff, W.W., and Revzan, K.L. (1986). Distribution of airborne radon-222 concentrations in U.S. homes, Science, 234, 992.

Nero, A.V., Revzan, K.L., and Sextro R.G. (1987). Appraisal of the U.S. data on indoor radon concentrations, Presented at the Fourth International Symposium on the Natural Radiation Environment, Lisbon, Portugal, December. Lawrence Berkeley Laboratory Report No.

LBL-24345.

Nitschke, I.A., Clarkin, M.E., Brennan, T., Rizzuto, J.E. and Osborne, M. (1988). Long-term assessment of residential radon-mitigation systems, Paper 88-107.5, in Annual Meeting of the Air Pollution Control Association, Dallas, Texas, June 19-24.

NJSDH (1989). A Case-Control Study of Radon and Lung Cancer among New Jersey Women (Technical Report - Phase I), New Jersey State Department of Health, Trenton, NJ.

Offerman, F.J., Sextro, R.G., Fisk, W.J., Grimsrud, D.T., Nazaroff, W.W., Nero, A.V., Revzan, K.L., and Yater, J. (1985). Control of respirable particles in indoor air with portable air cleaners, Atmos. Environ., 19, 1761.

Osborne, M.C. (1987). Resolving the radon problem in Clinton, New Jersey, houses, in Seifert et al. 1987 , p. 305.

Phillips, C.R., Khan, A. and Leung, H.M.Y. (1988). The nature and determination of the unattached fraction of radon and thoron progeny, in Nazaroff and Nero 1988, p. 203.

Poffijn, A., Marijns, R., Vanmarke, H., and Uyttenhuve, J. (1985). Results of a preliminary survey on radon in Belgium, Sci. Total Environ., 45, 335.

Porstendörfer, J. (1984). Behavior of radon daughter products in indoor air, Radiat. Prot. Dosim., 7, 107.

Porstendörfer, J., Wicke, A., and Schraub, A. (1978). The influence of exhalation, ventilation and deposition processes upon radon $\left({ }^{222} \mathrm{Rn}\right)$ and thoron $\left({ }^{220} \mathrm{Rn}\right)$ and their decay products in indoor air, Health Phys., 34, 465.

Prill, R.J., Fisk. W.J. and Turk, B.H. (1989). Evaluation of radon mitigation systems in 14 houses over a two-year period, Lawrence Berkeley Laboratory Report, LBL-27083, submitted to J. Air Pollution Cont. Assoc.

Puskin, J.S. and Nelson, C.B. (1989). EPA's perspective on risks from residential exposure, $J$. Air Pollution Cont. Assoc. 39, 915.

Put, L.W., and de Meijer, R.J. (1984). Survey of radon concentrations in Dutch dwellings, in Indoor Air: Radon, Passive Smoking, Particulates and Housing Epidemiology, Vol. 2, Berglund, B., Lindvall, T., and Sundell, J., Eds., Swedish Council for Building Research, Stockholm, p. 49. Radon Technical Information Service (1988). National Radon Measurement Proficiency (RMP) Program: Application and Participation Manual, Report EPA-520/1-88-056, U.S. Environmental Protection Agency, Washington, D.C. 
Raes, F. (1985). Description of the properties of unattached ${ }^{218} \mathrm{Po}$ and ${ }^{212} \mathrm{~Pb}$ particles by means of the classical theory of cluster formation, Health Phys., 49, 1177.

Raes, F., Janssens, A., Declerq, A., and Vanmarcke, H. (1984). Investigation of the indoor aerosol and its effect on the attachment of radon daughters, Radiat. Prot. Dosim., 7, 127.

Rannou, A. (1987). Indoor radon exposure assessment: an overview of the French survey, in APCA 1987, p. 78.

Reimer, G.M. and Gundersen, L.C.S. (1989). A direct correlation among indoor Rn, soil gas $\mathrm{Rn}$ and geology in the Reading Prong near Boyertown, Pennslyvania, Health Phys. 57, 155.

Revzan, K.L. (1989). Radon entry, distribution, and removal in two New Jersey houses with basements, submitted to Atmospheric Environment, Lawrence Berkeley Laboratory Report LBL-26830, Berkeley, CA.

Revzan, K.L., Nero, A.V., and Sextro, R.G. (1988). Mapping surficial radium content as a partial indicator of radon concentrations in U.S. houses, Radiat. Prot. Dosim., 24, 179.

Ronca-Battista, M., Moon, M., Bergsten, J., White, S.B., Alexander, B., and Holt, N. (1988). Radon 222 concentrations in the United States - results of sample surveys in five states, Radiat. Prot. Dosim. 24, 307.

Ronca-Battista, M., Magno, P. and Nyberg, P.C. (1988b). Standard measurement techniques and strategies for indoor ${ }^{222} \mathrm{Rn}$ measurements, Health Phys. 55, 67.

RPI (1986). Naturally Occurring Radiation in the Nordic Countries -.- Recommendations, The Radiation Protection Institutes in Denmark, Finland, Iceland, Norway and Sweden.

Rudnick, S.N. and Maher, E.F. (1986). Surface deposition of ${ }^{222} \mathrm{Rn}$ decay products with and without enhanced air motion, Health Phys., 51, 283.

Sachs, H.M., Hernandez, T.L., and Ring, J.W. (1982). Regional geology and radon in buildings Environ. Int., 8, 97.

Samuelsson, C. (1988). Retrospective determination of radon in houses, Nature 334, 338.

Schery, S.D. (1985). Measurements of airborne ${ }^{212} \mathrm{~Pb}$ and ${ }^{220} \mathrm{Rn}$ concentrations at varied indoor locations within the United States, Health Phys., 49, 1061.

Schery, S.D. (1986). Studies of thoron and thoron progeny: indications for transport of radioactivity from soil to indoor air, in APCA :986, p. 25.

Schery, S.D. (1986b). Private communication

Schery, S.D., Holford, D.J., Wilson, J.L., and Phillips, F.M., (1988). The flow and diffusion of radon isotopes in fractured porous media: part 1, finite slabs; part 2, semi-infinite media, Rad. Prot. Dos. 24, 185 and 191.

Schiller, G.E. (1984). A theoretical convective transport model of indoor radon decay products, Ph.D. thesis, University of California, Berkeley. 
Schiller, G.A., Nero, A.V., Revzan, K.L., and Tien, C.L. (1984). Radon decay-product behavior indoors: numerical modeling of convection effects. Lawrence Berkeley Laboratory Report LBL-17609, Berkeley, CA; see also Schiller et al. (1989), manuscripts submitted to Atmospheric Environ.

Schmeir, H., and Wicke, A. (1985). Results from a survey of indoor radon exposures in the Federal Republic of German, Sci. Total Environ., 45, 307.

Scott, A.G. (1983). Radon daughter deposition velocities estimated from field measurements, Health Phys., 45, 481.

Scott, A.G (1983b). The distribution of average radon daughter concentrations in houses estimated from single-sample surveys, Health Phys. 45, 435.

Scott, A.G. (1988). Preventing radon entry, in Nazaroff and Nero 1988, p. 407.

Scott, A.G. (1989). Effect of indoor radon variability on the duration and interpretation of radon measurements, in USEPA 1989 b, p. 4-15.

Seifert, B., Esdorn, H., Fischer, M., Rüden, H., and Wegner, J. (1987). Indoor Air '87, Proceedings of 4th International Conference on Indoor Air Quality and Climate (Berlin, 17-21 Aug. 1987), Institute for Water, Soil and Air Hygiene, Berlin, Volume 2, Environmental Tobacco Smoke, Multicomponent Studies, Radon, Sick Buildings, Odours and Irritants, Hyperractivities and Allergies.

Seinfeld, J.H. (1986). Atmospheric Physics and Chemistry of Air Pollution, Wiley: New York. Sexton, K. and Repetto, R. (1982). Indoor air pollution and public policy, Environ. Int. 8, 5. Sextro, R.G. and Lee, D.D. (1989). The performance of charcoal-based radon detection under time-varying radon conditions: experimental and theoretical results, in USEPA 1989b, p. 2-81.

Sextro, R.G., Harrison, J., Moed, B.A., Revzan, K.L., Turk, B.H., Grimsrud, D.T., Nero, A.V., Sanchez, D.C., and Teichman, K.Y. (1987). An intensive study of radon and remedial measures in New Jersey homes: preliminary results, in Seifert et al. 1987, p. 295.

Sextro, R.G., Nazaroff, W.W., and Turk, B.H. (1989). Spatial and temporal variation in factors governing the radon source potential of soil, in EPA 1989b, p. 5-61.

Sinnaeve, J., Clemente, G. and O'Riordan, M. (1984). The emergence of natural radiation, Radiat. Prot. Dosim., 7, 15.

Small, M.J. and Peters, C.A. (1988). Public policy model for the indoor radon problem, Mathl \& Comput. Modeling, 10, 5, p. 349-358.

Smith, D. (1979). Ventilation rates and their influence on equilibrium factor, in AECB 1979.

Ster, J.D. and Sexton, K. (1983). Indoor air pollution: a public health perspective, Science, $221,9$. 
Spitz, H. B., Wrenn, M.E., and Cohen, N. (1980) Diurnal variation of radon measured indoors and outdoors in Grand Junction, Colorado, and Teaneck, New Jersey, and the influence that ventilation has upon the buildup of radon daughters, in Natural Radiation Environment III, Gesell, T.F. and Lowder, W.M., Eds., US Dept. of Energy, CONF-780422, Springfield, VA, p. 1308.

Steck, D.J. (1989). Statewide radon surveys: screening vs. long-term measurements, in USEPA 1989 b, p. $1-67$.

Stranden, E. (1987). Radon 222 in Norweigen dwellings, in Hopke 1987, p. 70.

Stranden, E. (1988). Building materials as a source of indoor radon, in Nazaroff and Nero 1988, p. 113.

Subbarao, K. and Balcomb, J.D. (1988). Short-term energy monitoring (STEM)-summary of 1988 activities, Solar Energy Research Institute, Golden, CO.

Swedjemark, G.A. (1986). Swedish limitation schemes to decrease Rn daughters in indoor air, Health Phys., 51, 569.

Swedjemark, G.A. and Mjones, L., (1984a). Radon and radon daughter concentrations in Swedish homes, Rad. Prot. Dos., 7, 341.

Swedjemark, G.A. and Mjones, L. (1984b). Exposure of the Swedish population to radon daughters, in Indoor Air: Radon, Passive Smoking, Particulates and Housing Epidemiology, Vol. 2. Berglund, B., Lindvall, T., and Sundell, J., Eds., Swedish Council for Building Research, Stockholm, p. 37.

Thor, P.W. (1984). Personal communications.

Tommasino, L. (1980). Solid dielectric detectors with breakdown phenomena and their applications in radioprotection, Nucl. Inst. Meth., 173, 73.

Toohey, R.E., Essling, M.A., Rundo, J., and Hengde, W. (1984). Measurements of the deposition rates of radon daughters on indoor surfaces, Radiat. Prot. Dosim., 7, 143.

Toth, A. (1984). A simple field method for determination of ${ }^{220} \mathrm{Rn}$ and ${ }^{222} \mathrm{Rn}$ daughter energy concentrations in room air. Rad. Prot. Dos., 7, 247.

Traynor, G.W., Aceti, J.D., Apte, M.G., Smith, B.V., Green, L.L. Smith-Reiser, A., Novak, K.M., and Moses, D.O. (1989). Macromodel for assessing residential concentrations of combustion-generated pollutants: model development and preliminary predictions for $\mathrm{CO}, \mathrm{NO}_{2}$, and respirable suspended particles, Lawrence Berkeley Laboratory Report, LBL-23089.

Turk, B.H., Brown, J.T., Geisling-Sobotka, K., Grimsrud, D.T., Harrison, J., Koonce, J.F., and Revzan, K.L. (1986). Indoor air quality and ventilation measurements in 38 Pacific Northwest commercial buildiags, Lawrence Berkeley Laboratory Report LBL-21453, Berkeley, CA.

Turk, B.H., Prill, F.J., Fisk, W.J., Grimsrud, D.T., Moed, B.A., and Sextro, R.G. (1986). Radon and remedial action in Spokane River Valley residences: an interim report, Lawrence Berkeley Laboratory Report LBL-21399, Lawrence Berkeley Laboratory, Berkeley, CA. 
Turk, B.H., Harrison, J., Prill, R.J., and Sextro, R.G. (1987). Interim report on diagnostic procedures for radon control, Lawrence Berkeley Laboratory Report, LBL-23089.

Turk, B.H., Prill, R.J., Grimsrud, D.T., Moed, B.A., and Sextro, R.G. (1989a). Characterizing the occurrence, sources, and variability of radon in Pacific Northwest homes, Lawrence Berkeley Laboratory Report, LBL-26960, submitted to J. Air Poll. Cont. Assoc.

Turk, B.H., Prill, R.J., Fisk, W.J., Grimsrud, D.T. and Sextro, R.G. (1989b). Effectiveness of radon control techniques in 15 homes, Lawrence Berkeley Laboratory Report, LBL-26961, submitted to J. Air Poll. Cont. Assoc.

Tymen, G., Mouden, A., McLaughlin, J.P., Wasiolek, P., and Rannou, A. (1988). A comparison of the exposure response of French ard Irish radon detectors during field measurements in houses in Brittany, Radiat. Prot. Dosim. 24, 371.

Ulbak, K., Stenum, B., Sorensen, A., Majborn, B. Botter-Jensen, L., and Nielsen, S.P. (1988).

Results from the Danish indoor radiation survey, Rad. Prot. Dos. 24, 401.

United Nations Scientific Committee on the Effects of Atomic Radiation (1982). Ionizing

Radiation: Sources and Biological Effects, United Nations, New York.

U.S. Bureau of the Census (1987). Statistical Abstract of the United States: 1988, 108th Edition, Washington, D.C., Table 182.

U.S. Congress (1988). Indoor Radon Abatement Act of 1988. Title III of the Toxic Substances Control Act, PL 100-551.

U.S. Department of Health and Human Services (1982). The Health Consequences of Smoking: Cancer, U.S. Government Printing Office, Washington, D.C.

U.S. Department of Health and Human Services (1988). Monthly Vital Statistics Report 37(6), supplement.

USEPA (1986). A Citizen's Guide' to Radon: What It Is and What To Do About It, Report OPA-86-004, United States Environmental Protection Agency and U.S. Department of Health and Human Services, U.S. Government Printing Office, Washington, D.C.

USEPA (1987). EPA Finds Radon Problems in 10-State Survey, EPA news release arid fact sheets, Washington, D.C. Aug. 4.

USEPA (1988). EPA and Assistant Surgeon General call for radon home testing, EPA news release and fact sheets, Washington, D.C., September 12.

USEPA (1989a). EPA urges schools to check for radon, EPA news release and fact sheets, Washington, D.C., April 20.

USEPA (1989b). Proceedings: The 1988 Symposium on Radon and Radon Reduction Technolog.y. Vol. 1 - the Symposium Oral Papers, Report EPA-600/9-89-006a.

USGAO, U.S. General Accounting Office (1989). Air Pollution: Uncertainty Exists in Radon Measurements, report GAO/RCED-90-25, Washington, DC. 
Vitz, E. (1989). Preliminary results of a nationwide waterborne radon survey, in EPA 1989b, p. $8-1$.

WHO (1987). Air Quality Guidelines for Europe, WHO Regional Publications, European Series No. 23, World Health Organization Regional Office for Europe, Copenhagen.

Wicke, A. (1979). Untersuchengen zur Frage der Naturlichen Radioktivitat der Luft in Wohnand Aufenthaltstraumen, Phd., thesis, Justus Liebig Universitat, Giessen.

Wilkening, M.H., Clements, W.E., and Stanley, D. (1972). Radon-222 flux measurements in widely separated regions, in Natural Radiation Environment II, Adams, J.A.S., Lowder, W.M., and Gesell, T.F., Eds., CONF-720805, National Technical Information Service, Springfield , VA, p. 717.

Wollenberg, H.A. and Smith, A.R. (1987). Radiogenic heat production of crustal rocks, Geophys. Res. Lett., 14, 295

Wollenberg, H.A. and Revzan, K.L. (1989). Radium regionalization in California, submitted to Geophys. Res. Lett.

Yokel, F.Y. (1989). Site characterization for radon source potential, U.S. Department of Cummerce report NISTIR 89-4106, Gaithersburg, MD. 


\section{APPENDICES}

\section{A. RADIOACTIVE QUANTITIES AND UNITS OF MEASURE}

Amounts of radioactive material can be specified in principle by either mass or activity, the latter being more conventional. The activity is the actual rate at which atoms decay radioactively, and the standard international (SI) unit for activity is the becquerel (Bq), equal to a decay rate of one per second $\left(\mathrm{s}^{-1}\right)$. This unit (or its traditional equivalent, the curie) is adequate for expressing activity and (activity) concentration, not only for radon, but also for its decay products and any combination of them. The present work utilizes SI units primarily, but traditional units are defined below in terms of he modern units. In the text, the older units are sometimes given parenthetically or when citing work that is difficult to translate without modifying sense or quantitative results.

For decay products, the collective quantily of most use is the equilibrium-equivalent decayproduct concentration (EEDC). Based on the alpha decay energies and half lives of the

${ }^{222} \mathrm{Rn}$ decay series (see Figure 2), the EEDC is given in terms of the individual decayproduct concentrations as:

$$
\mathrm{EEDC}=0.104 \times \mathrm{I}\left({ }^{218} \mathrm{Po}\right)+0.514 \times \mathrm{I}\left({ }^{214} \mathrm{~Pb}\right)+0.382 \times \mathrm{I}\left({ }^{214} \mathrm{Bi}\right),
$$

with concentrations of each decay product (and of the EEDC) given in $\mathrm{Bq} / \mathrm{m}^{3}$ or $\mathrm{pCi} / \mathrm{l}$, as indicated in Table 10. (The ${ }^{214}$ Po concentration does not contribute significantly to this expression because of its very short half life, causing very few ${ }^{214}$ Po atoms to be present in air as compared with the other short-lived decay products. However, the ${ }^{214}$ Po alpha energy is the largest contributor to the coefficients in this expression, since the presence of each of the previous three decay products implies a ${ }^{214}$ Po decay.) The analogous expression for the ${ }^{220} \mathrm{Rn}$ series (cf. data in Appendix $\mathrm{D}$, below) is

$$
\mathrm{EEDC}=0.913 \times \mathrm{I}\left({ }^{212} \mathrm{~Pb}\right)+0.087 \times \mathrm{I}\left({ }^{212} \mathrm{Bi}\right) .
$$

$\left({ }^{216}\right.$ Po and ${ }^{212}$ Po do not contribute directly to this expression - again, because of their very short half lives - even though ${ }^{212}$ Po contributes the dominant alpha energy.)

Historically, the potential alpha energy concentration (PAEC) itself was used as the measure of decay-product concentration, with the standard unit being working level (WL). The corresponding SI unit is that of energy per unit volume, $\mathrm{J} / \mathrm{m}^{3}$, and: $\mathrm{PAEC}=\mathrm{EEDC} \times 5.56$ $\times 10^{-9} \mathrm{~J} / \mathrm{Bq}$ (or $1283 \mathrm{MeV} / \mathrm{pCi}$ ) for the ${ }^{222} \mathrm{Rn}$ series. (The corresponding factor for the ${ }^{220} \mathrm{Rn}$ series is $7.56 \times 10^{-8} \mathrm{~J} / \mathrm{Bq}$ or $1.75 \times 10^{4} \mathrm{MeV} / \mathrm{pCi}$.) In any case, the PAEC is effectively supplanted by the EEDC (i.e., the decay-product concentration itself), except possibly in specifying exposures. (Note that in one respect the PAEC is a more effective measure of concentration than the EEDC, i.e., in comparing ${ }^{222} \mathrm{Rn}$ and ${ }^{220} \mathrm{R} n$ : per unit radioactivity, the ${ }^{220} \mathrm{Rn}$ decay products carry 13.6 times as much i'AEC as the ${ }^{222} \mathrm{Rn}$ decay products.)

The basic measure of exposure is essentially the product of concentration (or its equivalent) and time. EEDC $\times$ time has units of $\mathrm{Bq} \mathrm{m}^{-3} \mathrm{~s}$, although hour, month, or year might also be used as the unit of time. PAEC $\times$ time has SI units of $\mathrm{J} \mathrm{m}^{-3}$, but the traditional units are working-level hour (WLH) or - - more frequently - - working-level month (WLM), equal to 173 WLH. Exposure rate, in the SI units, simply reduces to average EEDC (or alternatively PAEC), but in the old units is usually $\mathrm{WLM} / \mathrm{y}$. 
Table 10. SI Units and Equivalents for Traditional Units ${ }^{a}$

\section{Parameter, SI Unit}

Activity, Bq

Concentration, $\mathrm{Bq} \mathrm{m}^{-3}$

PAEC, $\mathrm{J} \mathrm{m}^{-3}$

$\mathrm{EEDC}_{222}, \mathrm{~Bq} \mathrm{\textrm {m } ^ { - 3 }}$

$\mathrm{EEDC}_{220} \mathrm{~Bq} \mathrm{~m}-3$

Exposure, $\mathrm{J} \mathrm{m}^{-3} \mathrm{~S}$

Exposure, $\mathrm{Bq} \mathrm{m}^{-3} \mathrm{y}$

Exposure rate, $\mathrm{J} \mathrm{m}^{-3}$

Exposure rate, $\mathrm{Bq} \mathrm{m} \mathrm{m}^{-3}$

\section{Conversion for Traditional Unit}

$1 \mathrm{Ci}=3.7 \times 10^{10} \mathrm{~Bq}(1 \mathrm{pCi}=0.037 \mathrm{~Bq})$

$1 \mathrm{pCi} / 1=37 \mathrm{~Bq} \mathrm{~m}^{-3}$

$1 \mathrm{WL}=1.3 \times 10^{5} \mathrm{MeV} / 1=2.08 \times 10^{-6} \mathrm{~J} \mathrm{~m}^{-3}$

$1 \mathrm{WL}(\mathrm{PAEC})=3750 \mathrm{~Bq} \mathrm{~m}^{-3}$

$1 \mathrm{WL}(\mathrm{PAEC})=276 \mathrm{~Bq} \mathrm{~m}-3$

$1 \mathrm{WLM}=12.97 \mathrm{~J} \mathrm{~m}^{-3} \mathrm{~S}$

$1 \mathrm{WLM}=74.0 \mathrm{~Bq} \mathrm{~m}^{-3} \mathrm{y}$ (for the ${ }^{222} \mathrm{Rn}$ series)

$1 \mathrm{WLM} / \mathrm{y}=4.11 \times 10^{-7} \mathrm{~J} \mathrm{~m}^{-3}$

$1 \mathrm{WLM} / \mathrm{y}=74.0 \mathrm{~Bq} \mathrm{~m}-3$ (for the ${ }^{222} \mathrm{Rn}$ series)

a The data on which these conversions and the expressions for EEDC are based are taken from Browne and Firestone (1986), except for the half-life of ${ }^{218} \mathrm{Po}$, taken to be the 3.04 min found by Martz et al. (1989). 


\section{B. VENTILATION RATES: DISTRIBUTION AND DEPENDENCE}

A key factor affecting indoor pollutant concentrations is the ventilation rate. Characterizing ventilation rates in building can be difficult, since many buildings are complex structures, and paths and rates of air movement can depend substantially on location and time. Even considering only homes, the question is complicated, both because of the wide variety of housing structures and because - even for a given (and even simple) structure type - the pattern of air movement is complex. These complexities have substantial implications for the behavior of any reactive species, including radon decay products, and for the manner and degree of radon entry, as described elsewhere in this report.

Nonetheless, restricting attention to homes - consisting of relatively self-contained living units - simplifies the picture sufficiently that the ventilation rate has a less ambiguous meaning (although even this can be complicated in the case of multi-apartment buildings in which air is recirculated among different units). Moreover, the rate can actually be measured relatively directly. For example, the ventilation rate measurements contributing to Figure 3 were performed by injecting a tracer gas, sulfur hexafluoride $\left(\mathrm{SF}_{6}\right)$, into each house, then - after a mixing time - measuring the concentration as a function of time using an infrared analyzer: the ventilation rate equals the rate of decay of the tracer concentration. And in a current passive measurement technique, a tracer is released from a small source at a constant rate and a collecting monitor, consisting of a diffusion tube and an absorber (characteristic of a number of techniques for passive sampling of gaseous pollutants indoors), measures the average concentration during the time this system is deployed (Dietz and Cote 1982): this measured value is then proportional, in first order, to the average of the inverse of the ventilation rate, $I_{\text {tracer }}=S_{v}\left(1 / \lambda_{v}\right)_{\text {average }}$, where $S_{v}$ is the source's release rate per unit volume.

The results of such measurements confirm the expectation that ventilation rates vary substantially from one country to another, from one class of buildings to another, and even within the same general structural class, e.g., single-family houses or multi-unit apartment buildings. Still, for most homes in the United States (and many industrialized nations), the average ventilation rate during seasons when the windows are kept mostly closed lies in the range $0.1-1 \mathrm{~h}^{-1}$. This total ventilation rate is made up of three components: infiltration of air through small openings or imperfections in the building shell, exchange of air through windows or doors that are partially or temporarily open, and ventilation supplied mechanically by exhaust fans or other systems. Each of these components varies with time, not only from one season to another, but also from one day to the next, and even over shorter periods. As a result, the total ventilation rate has a significant time dependence, even within a single building. Even during the heating season, when - for many homes infiltration supplies most of the ventilation, substantial variability occurs, as noted below.

It is important, in considering the results of ventilation rate measurements, to pay attention to what component of the ventilation the monitoring protocol was designed to measure. For example, the basic tracer injection technique mentioned above is of ten employed with windows closed, therefore measuring only the infiltration rate, of ten the dominant winter component. In contrast, the integrating passive technique measures the average inverse total ventilation rate.

Like the radon concentrations discussed in this report, measurements of infiltration rate in any sample of buildings are of ten found to be distributed lognormally, i.e., the distribution of the log of the ventilation rate is approximately "normal" or Gaussian. An example of this is a set of measurements taken in 200 single-family houses in several cities of the United States, where weatherization programs were taking place (Grot and Clark 1981). The average infiltration rate was found to be $1.1 \mathrm{~h}^{-1}$, and a more recent determination of 
the geometric mean and standard deviation yields 0.90 and 2.13 , respectively, as discussed elsewhere (Nazaroff et al. 1987b). Considering the age and condition of these houses, their infiltration rates are higher than the average U.S. value, which in turn is significantly higher than in northern European countries where substantial energy-saving efforts appear to have effected significant reduction in infiltration rates.

In recent years, the processes driving infiltration rates in homes have come to be better understood, and even embodied in relatively simple quantitative models. Basically, we now know that infiltration occurs because of air convection into and out of the house, driven by small pressures across the building shell arising from two factors: winds, which obviously exert small - albeit complex - forces on the building and its surroundings (specifically, the ground), and temperature differences between indoors and outdoors. During the heating season, this temperature difference causes a "stack" effect (much as in a fireplace and chimney), such that air is drawn in near the bottom of the structure and forced uut toward the top. The pressure differences caused by winds and temperature differences are roughly comparable in size, averaging on the order of a few pascal (with higher values in relatively severe climates). Because the size and pattern of the associated pressures vary markedly from one time to another, it is not surprising that infiltration rates also vary substantially.

A number of groups have formulated models giving the infiltration rates in terms of the pertinent environmental parameters, and their success is reviewed in a publication of the Air Infiltration Centre (Liddament and Allen 1983). As successful as any model - even complex ones - is a simple parameterization of the infiltration rate in terms of the wind speed $\mathrm{V}$, the temperature difference $\Delta T$, and an effective "leakage area" $A_{0} \cdot$ In these terms, the infiltration component of the ventilation rate may be expressed as

$$
v_{i}=A_{o}\left[\left(f_{w} V\right)^{2}+\left(f_{s} \Delta T^{1 / 2}\right)^{2}\right]^{1 / 2}
$$

here $f_{w}$ is a parameter accounting for local and terrain shielding effects, the distribution of leakage area around the building envelope, and the height of the building relative to the height at which the wind speed is measured; $f$ is a stack parameter accounting for the building height and the distribution of leakage area (Grimsrud et al. 1982). Using this model together with applicable meteorological information, heating-season infiltration rates have been estimated from measurements performed in 200 houses distributed throughout the United States and Canada. The average was found to be $0.67 \mathrm{~h}^{-1}$ (with a significantly lower value, $0.48 \mathrm{~h}^{-1}$, found for houses less than two years old at the time of measurement). 


\section{OUTDOOR CONCENTRATIONS OF RADON AND ITS DECAY PRODUCTS}

During the last two or three decades, a significant effort has been devoted to monitoring of outdoor radon concentrations, either as part of the overall characterization of environmental radiation exposures or to determine environmental radon levels as a contrast to those in occupational or indoor settings. A paper by Gesell reviews and analyzes the results from such efforts, examining the variability of outdoor ${ }^{222} \mathrm{Rn}$ concentrations with time of day, season, altitude, and location (Gesell 1983).

The magnitude and behavior of outdoor concentrations is indicated by Figure 23, where continuous measurements taken at three locations are seen to yield concentrations (averaged over approximately one month) in the range of 4 to $15 \mathrm{~Bq} / \mathrm{m}^{3}(0.1-0.4 \mathrm{pCi} / \mathrm{l})$, with a significant seasonal variation whose behavior differed among the locations. Examining the same data versus time of day yields average concentrations having a substantial diurnal variability (roughly a factor-of-two range in each case), with the lowest values occurring at noon or soon thereafter and the highest in the middle of the night or early morning. The observed variation with time can be attributed to differences in environmental conditions, affecting not only the movement of radon out of the ground, but also - and probably more importantly - the rapidity of mixing in the atmosphere. This mixing determines the height dependence of concentration, which varies only modestly over distances comparable to the height of buildings (e.g., about a factor of two from 1 to $100 \mathrm{~m}$ or from 0.01 to $1 \mathrm{~m}$ height above the ground).

Significantly higher and lower values than found in Figure 23 have been found at other locations, e.g., averages of $28 \mathrm{~Bq} / \mathrm{m}^{3}$ in Grand Junction and $0.5-1 \mathrm{~Bq} / \mathrm{m}^{3}$ in Alaska; low values also occur in areas substantially influenced by marine air. Moreover, instantaneous values can also vary markedly from the averages. Gesell suggests that the mean value for the contiguous United States lies between 0.1 and $0.4 \mathrm{pCi} / 1\left(4-15 \mathrm{~Bq} / \mathrm{m}^{3}\right)$, probably in the vicinity of $0.25 \mathrm{pCi} / 1\left(10 \mathrm{~Bq} / \mathrm{m}^{3}\right)$. Data from other countries are not dissimilar.

Some information is also available on the decay products in outdoor air, where - because of the relative absence of surfaces (compared with indoors) - one might expect higher equilibrium ratios. For example, measurements in New York yielded ratios around 0.45 (with uncombined fraction of ${ }^{218}$ Po of $9 \%$ ) at a rural location and 0.40 (uncombined fraction of $5 \%$ ) on a city sidewalk (George 1972). Subsequent measurements outside eight houses in New York and New Jersey gave mean equilibrium ratios averaging 0.79 , considerably higher than the indoor ratios measured (George and Breslin 1980). More recent data (cf. Table 11) yields an equilibrium factor of 0.56 .

A ${ }^{222} \mathrm{Rn}$ concentration of $10 \mathrm{~Bq} / \mathrm{m}^{3}$, while completely negligible compared with the higher indoor values observed, is still significant compared with typical indoor concentrations. Therefore the outdoor contribution has to be considered in making precise estimates of human exposures to ${ }^{222} \mathrm{Rn}$ decay products. It also has to be considered in examining the sources of radon found indoors: the approximately $10 \mathrm{~Bq} / \mathrm{m}^{3}$ coming in with outdoor air is about $20 \%$ of the average ${ }^{222} \mathrm{Rn}$ concentration observed in U.S. single-family homes and an even larger percentage of concentrations in other types of buildings. 


\section{SEASONAL VARIATION}

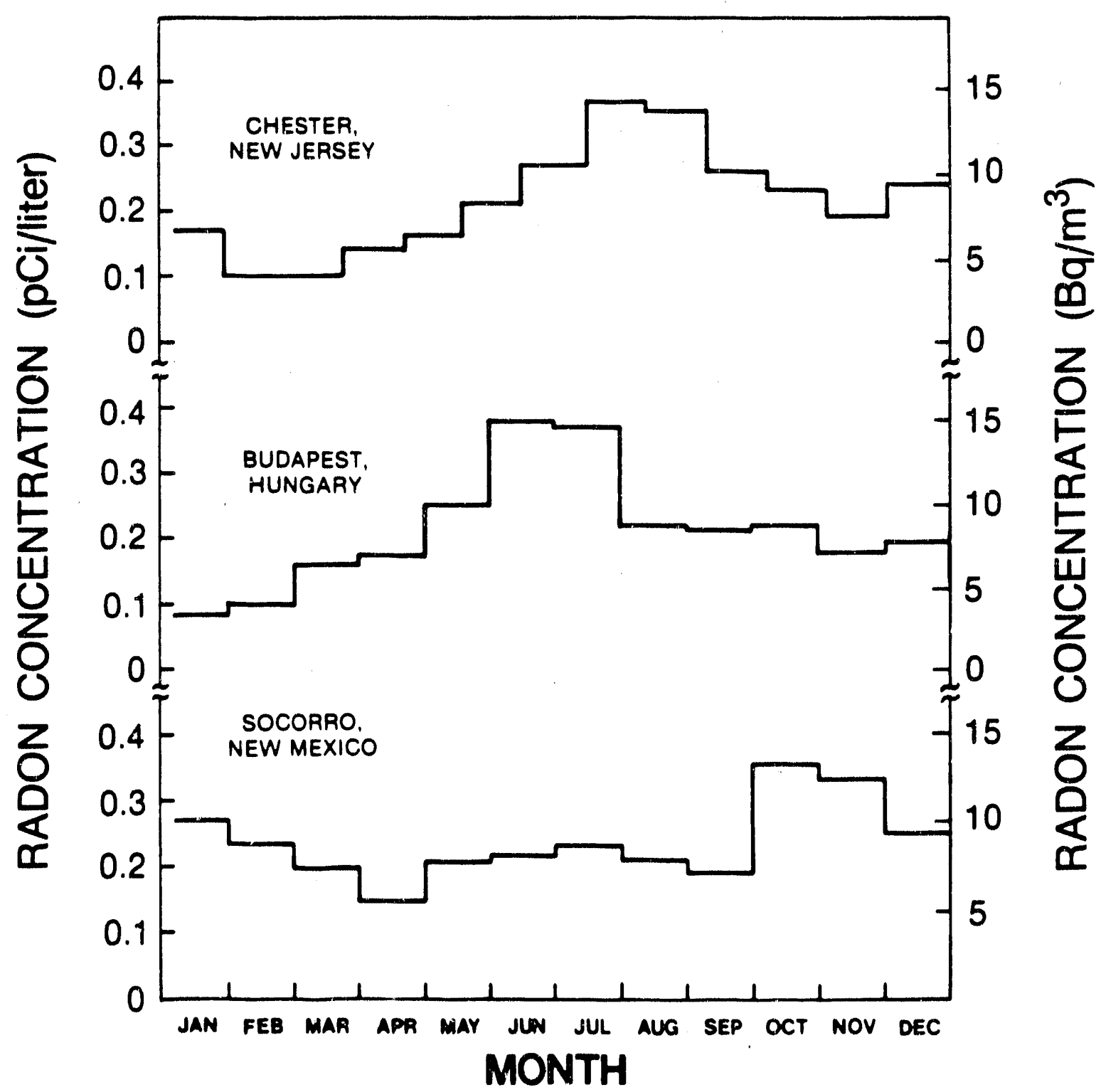

XBL 871.9911

Figure 23. Year-long variability of atmospheric ${ }^{222} \mathrm{Rn}$ concentration.

Measurements were performed $1 \mathrm{~m}$ above the ground in three different locations (figure adapted from Gesell (1983), by permission). 


\section{RADON 220 AND ITS DECAY PRODUCTS}

Our present understanding of the occurrence and behavior of ${ }^{220} \mathrm{R} n$ and its decay products is very limited compared with our knowledge of the ${ }^{222} \mathrm{Rn}$ series. Not nearly as much effort has been devoted to ${ }^{220} \mathrm{Rn}$ characterization, because preliminary and modest evidence - both theoretical and experimental - suggests that airborne concentrations of ${ }^{220} \mathrm{Rn}$ and its decay products are not as important from a radiological point of view as their more common relatives. This occurs in spite of the fact that the ${ }^{232} \mathrm{Th}$ decay series, shown in Figure 24, has approximately the same (activity) concentration in the earth's crust as the ${ }^{238} \mathrm{U}$ series, about $25 \mathrm{~Bq} / \mathrm{kg}$ (Nero 1983, United Nations 1982).

The differences have to do partly with the short half life of ${ }^{220} \mathrm{Rn}, 1 \mathrm{~min}$ as compared with $4 \mathrm{~d}$ for ${ }^{222} \mathrm{Rn}$, and partly with the details of radon and decay product behavior in the indoor or outdoor environment and inside the lung. The first factor sharply limits the time available for transport from the source material, so that only a small thickness of building material or soil serves as an effective source for ${ }^{220} \mathrm{Rn}$ entering the atmospheric environment, indoors or outdoors. The second affects the concentrations present in the air and the dose delivered by the decay products that deposit in the lung. The net result of all these factors is that - at least for indoor ${ }^{222} \mathrm{Rn}$ concentrations in the normal range - the ${ }^{220} \mathrm{Rn}$ decay products (in particular ${ }^{212} \mathrm{~Pb}$ ) appear - on the basis of limited evidence - to have a potential alpha energy concentration (PAEC) that is less than, but still significant compared with, that from the ${ }^{222} \mathrm{Rn}$ series. However, the PAEC itself does not reflect the present understanding that a given amount of alpha energy from the ${ }^{222} \mathrm{R} n$ series has substantially more biological impact than alpha energy from the ${ }^{220} \mathrm{Rn}$ series (cf. James 1988).

We consider first the concentrations expected if transport from the source to the air were dominated by diffusion for both of these radon isotopes, as is likely to be the case outdoors. The depth of soil serving as a source is then indicated by the diffusion length, which is proportional to the square root of the half life. The ratio of the ${ }^{220} \mathrm{Rn}$ to ${ }^{222} \mathrm{Rn}$ half lives is $1 / 5940$ (i.e., $55.6 \mathrm{~s} / 3.82 \mathrm{~d}$ ), yielding $1 / 77$ as the diffusion length ratio. This indicates the relative depth cleared per characteristic time, which is given by the half lives. Since the activity concentration in the soil is similar for the ${ }^{232} \mathrm{Th}$ and ${ }^{238} \mathrm{U}$ series, the relative rate at which ${ }^{220} \mathrm{Rn}$ and ${ }^{222} \mathrm{Rn}$ activity escape from the surface of the source material is the relative thickness cleared $(1 / 77)$ divided by the relative time scale $(1 / 5940)$, yielding a factor of 77 . Thus much more ${ }^{220} \mathrm{R} n$ activity is expected to escape than ${ }^{222} \mathrm{Rn}$ activity! (However, because of the difference in half lives, this greater activity is supported by oniy $1 / 77$ th as many atoms.) In contrast, it is easy to show that - if the transport mechanism is pressure-driven flow of air through the source material and into the open air - the rate at which activity leaves the surface is the same for the two isotopes. (In this case, only $1 / 5940$ th as many ${ }^{220} \mathrm{Rn}$ atoms escape.)

For a typical ventilation rate of $1 \mathrm{~h}^{-1}$, the assumption of pure diffusion (with no barriers between the source and the air) can therefore be shown, using Eq. 1 of Section 1, to imply about twice as much ${ }^{220} \mathrm{Rn}$ activity in indoor air as ${ }^{222} \mathrm{Rn}$ (since the ratio of activities entering is similar to the ratio of the ventilation rate to the ${ }^{220} \mathrm{Rn}$ decay constant). As we will see, the observed ratio is not usually this large. However, for outdoor air, the correspondence is reasonably good. For example, a review by Schery (1986) of ${ }^{220} \mathrm{Rn}$ and its decay products indoors cites typical fluxes from open soil of $2 \mathrm{~Bq} \mathrm{~m}^{-2} \mathrm{~s}^{-1}$ for ${ }^{220} \mathrm{Rn}$ and $0.016 \mathrm{Ba} \mathrm{m} \mathrm{m}^{-2} \mathrm{~s}^{-1}$ for ${ }^{222} \mathrm{Rn}$, a ratio that is only slightly larger than the factor of 77 suggested above. Based on a simple eddy diffusion model to represent the transport of radon upward into the atmosphere, he concludes that the concentrations of ${ }^{220} \mathrm{Rn}$ and ${ }^{222} \mathrm{Rn} 1 \mathrm{~m}$ above the ground ought to be 9 and $6 \mathrm{~Bq} / \mathrm{m}^{3}$, respectively, compared with his (limited) measurement results of 16 and $6 \mathrm{~Bq} / \mathrm{m}^{3}$, respectively. He notes that his tentative result of $4.6 \mathrm{~nJ} / \mathrm{m}^{3}$ for $\overline{P A E C}{ }_{212}$ (the PAEC from ${ }^{212} \mathrm{~Pb}$, the primary ${ }^{220} \mathrm{Kn}$ decay product: see Appendix $\mathrm{A}$ on 


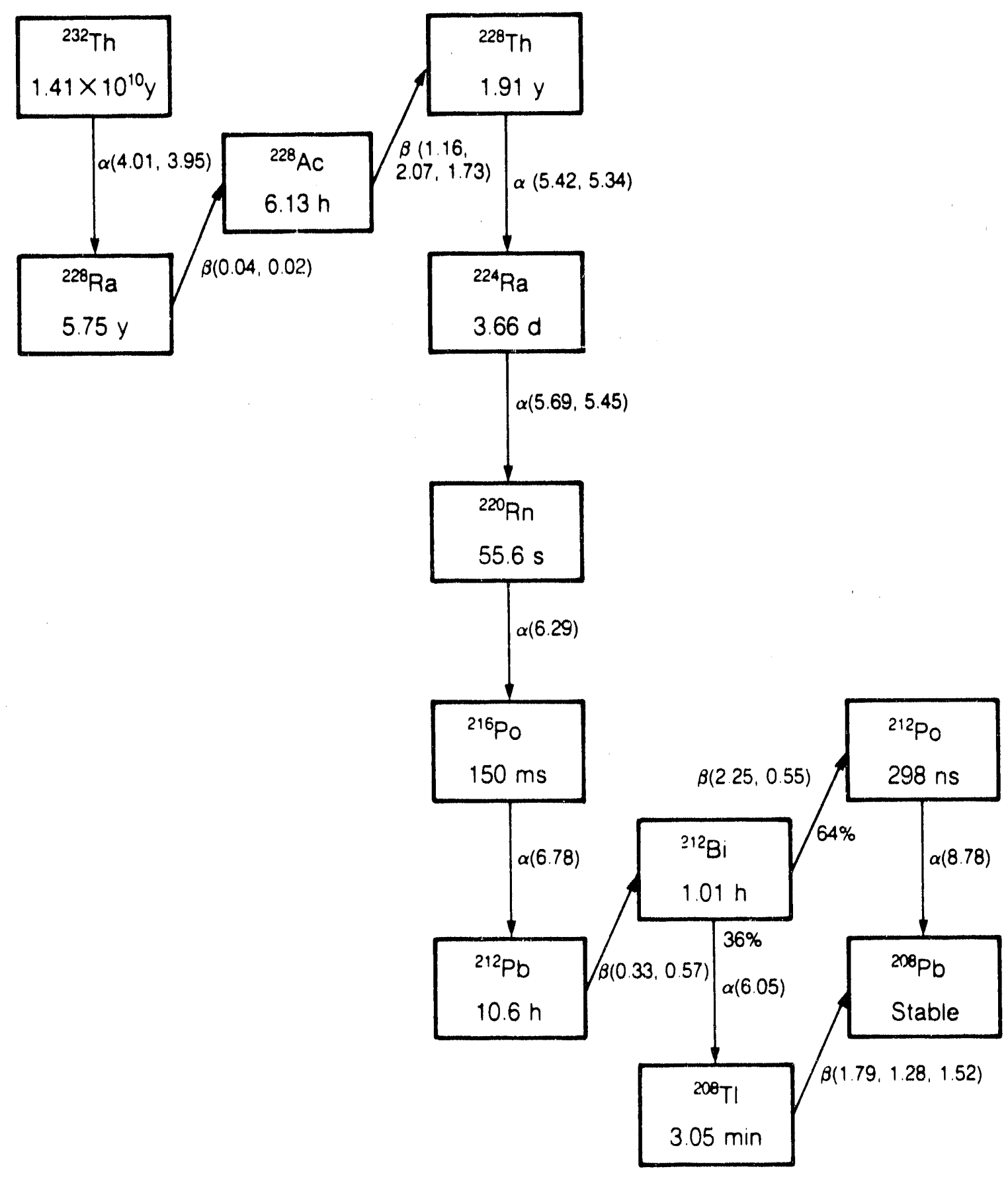

XBL 871.9208

Figure 24. Thorium 232 decay chain, including ${ }^{220} \mathrm{Rn}$ and its decay products.

Radon 220 and its parent, ${ }^{224} \mathrm{Ra}$, are members of the ${ }^{232} \mathrm{Th}$ decay series. Airborne concentrations of ${ }^{212} \mathrm{~Pb}$ and ${ }^{212} \mathrm{Bi}$ are of prime radiological interest due to their potential for retention in the lung, leading to subsequent irradiation by the alpha decay of ${ }^{212} \mathrm{Po}$. Cf. Figure 2 for ${ }^{238} \mathrm{U}$ decay chain. Half-lives and alpha energies (in MeV) taken from Browne and Firestone (1986); beta end-point energies (in MeV) are from NCRP (1985). 
quantities and units), versus $20 \mathrm{~nJ} / \mathrm{m}^{3}$ for $\mathrm{PAEC}_{222}$ (the PAEC from the ${ }^{222} \mathrm{Rn}$ decay products), compares reasonably with more extensive measurements made in the Federal Republic of Germany (Keller et al. 1982). (These results imply an equilibrium factor for ${ }^{220} \mathrm{Rn}$ decay products that is extremely small -- not surprising considering the relative half lives of ${ }^{220} \mathrm{Rn}$ and ${ }^{212} \mathrm{~Pb}$-- and an equilibrium factor of ${ }^{222} \mathrm{Rn}$ decay products greater than 0.5.)

The data on indoor concentrations are limited and more difficult to interpret. A number of workers cite results for the ratio of $\mathrm{PAEC}_{220}$ to $\mathrm{PAEC}_{222}$ centering around 0.5 . See, for example, European data ranging from 0.3 to 0.8 , summarized in Toth (1984) and North American results from Elliott Lake, Canada, of 0.3 (Gunning and Scott 1982) and for the United States of 0.6 (Schery 1985). These results indicate the potential significance of the ${ }^{220} \mathrm{Rn}$ series indoors. However, our understanding of the factors affecting the ${ }^{220} \mathrm{R} . \mathrm{n}$ concentrations, both absolutely and relative to ${ }^{222} \mathrm{Rn}$, is tentative at best.

For example, if entry of both radon isotopes is diffusion dominated, as has been supposed in some classes of European housing (particularly apartment structures), and concentrations in the source materials are similar, then indoor radon concentrations ought to be similar for the two isotopes (as noted earlier), the activity concentration of the decay products ought to be a factor of 5 or so less for ${ }^{220} \mathrm{Rn}$ than for ${ }^{222} \mathrm{Rn}$ (because the long half life of ${ }^{212} \mathrm{~Pb}$ causes most of it to be removed by ventilation or deposition rather than by decay), and the PAEC $_{220}$ ought to be greater than the PAEC ${ }_{222}$ by a modest factor, e.g., 2 or 3 . Even in European housing the ratio is closer to 0.5 , suggesting significant differences in the source concentrations or generation parameters for the two isotopes, barriers to the presumed diffusion process, other mechanisms for radon entry, or unaccounted-for differences in the decay-product behavior.

One difficulty is obvious, i.e., at the concentrations typical of European apartment structures, the ${ }^{222} \mathrm{Rn}$ entering the indoor air from structural materials can almost be equaled by that entering from the outdoor air. In contrast, the ${ }^{220} \mathrm{Rn}$ half life is so short compared with ventilation time constants that no comparable contribution arises for this isotope.

Another factor to be considered is the more interesting one that pressure-driven flow has been shown to be the dominant contributor to indoor ${ }^{222} \mathrm{Rn}$ for many types of structures and may also be significant for ${ }^{220} \mathrm{Rn}$. For homes such as those that dominate thu U.S. housing stock, little diffusion from the floor and wall material is expected in any case (because of the use of wood and the painting of other types of materials), so that some other entry route is likely to dominate observed concentrations of the ${ }^{220} \mathrm{Rn}$ series. As indicated above, comparable concentrations in the source materials would imply similar activity entry rates for a pure flow process. Because of the relative size of decay rates and typical ventilation rates, this would imply ${ }^{220} \mathrm{R} n$ concentrations only about $2 \%$ of ${ }^{222} \mathrm{Rn}$ concentrations and (using the same argument as above) a similar ratio of PAECs, if we ignore the concentration gradients possible for such a short-lived isotope as ${ }^{220} \mathrm{Rn}$. Thus the concentration ratios expected differ by about two orders of magnitude, depending on whether diffusion or flow is the presumed mechanism, and the actual observations - in both European and American housing - lies approximately at the geometric mean. This suggests the dominance of different entry mechanisms for the two isotopes or a combination of diffusion and flow applying to both, a possibility that was inclicated in the early work on pressure-driven flow (Nazaroff et al. 1985).

However, the measurements of Schery in U.S. buildings (Schery 1985) also afford the possibility for an interesting comparison, indicated in Table 11. There are indicated the results of outdoor measurements cited above, as well as the average indoor PAEC $_{212}$ and PAEC $_{222}$ from Schery's 68 measurements in buildings in 21 states. The detailed data show a significant correlation between these two parameters (in contrast to the work of Gunning 
and Scott 1982), suggesting the possibility of common entry mechanisms (or, of course, common removal mechanisms). Also shown are estimates of the indoor radon concentrations, based on the PAEC ${ }_{222}$ for ${ }^{222} \mathrm{Rn}$ and on one of Schery's illustrations for the ${ }^{220} \mathrm{Rn}$. These concentrations are in good agreement with his average ${ }^{220} \mathrm{Rn} /{ }^{222} \mathrm{Rn}$ ratio of 0.23 , which is - consistent with the comments above - at the (geometric) midpoint between the expectations for purely diffusion and purely flow entry mechanisms.

However, the ${ }^{222} \mathrm{Rn}$ concentration estimate is considerably less than the average concentration in U.S. single-family homes suggested on the basis of the studies discussed in the body of this chapter, i.e., $55 \mathrm{~Bq} / \mathrm{m}^{3}$. This estimate may be so low because the equilibrium factor during Schery's measurements was considerably lower than the 0.4-0.5 assumed in converting his mean $\mathrm{PAEC}_{222}$ to a ${ }^{222} \mathrm{Rn}$ concentration (cf. Table 11). A mean equilibrium factor of 0.15 (Schery 1986) for these measurements yields, instead of 15-20 $\mathrm{Bq} / \mathrm{m}^{3}$, an estimated mean ${ }^{222} \mathrm{Rn}$ concentration near $50 \mathrm{~Bq} / \mathrm{m}^{3}$, consistent with other U.S. results. We are then left with the puzzle as to why the equilibrium factor for the ${ }^{222} \mathrm{Rn}$ decay products was so low compared with means found in other studies.

Furthermore, it is interesting to note the relative sizes of the PAECs cited in the table. The indoor PAEC P22 is clearly the largest, and the indoor $\mathrm{PAEC}_{212}$ is comparable to the outdoor PAEC $_{222}$. Since - as noted in Schery (1986) and discussed more throughly in James (1988) a given PAEC from the ${ }^{220} \mathrm{Rn}$ series is thought to have much less health significance than a comparable PAEC from the ${ }^{222} \mathrm{Rn}$ decay products, it appears that indoor concentrations of the 220 series are less significant even than outdoor radon concentrations. Nonetheless, the possible health significance is not to be ignored, and - indeed - understanding better the behavior of the ${ }^{220} \mathrm{Rn}$ series presents not only an interesting scientific problem, but even a tool by which to explore more fully the mechanisms for transport of the radon isotopes into the indoor environment.

Table 11. Comparative Concentrations of ${ }^{220} \mathrm{Rn}$ and ${ }^{222} \mathrm{Rn}$ and Their Decay Products

\begin{tabular}{|c|c|c|c|c|}
\hline & \multicolumn{2}{|c|}{$\begin{array}{c}\text { Outdoor } \\
\text { (1 m height) }\end{array}$} & \multicolumn{2}{|c|}{ Indoor } \\
\hline & $\begin{array}{c}\mathrm{Rn} \\
\left(\mathrm{Bq} / \mathrm{m}^{3}\right)\end{array}$ & $\begin{array}{c}\text { PAEC } \\
\left(\mathrm{nJ} / \mathrm{m}^{3}\right)\end{array}$ & $\begin{array}{c}\mathrm{Rn} \\
\left(\mathrm{Bq} / \mathrm{m}^{3}\right)\end{array}$ & $\begin{array}{c}\text { PAEC } \\
\left(\mathrm{nJ} / \mathrm{m}^{3}\right)\end{array}$ \\
\hline${ }^{220} \mathrm{Rn}$ Series & 16 & $5^{b}$ & $3-4^{c}$ & $21^{b}$ \\
\hline${ }^{222} \mathrm{Rn}$ Series & 6 & 20 & $15-20^{d}$ & 44 \\
\hline
\end{tabular}

a Based on Schery (1985).

b These are only the PAEC's for ${ }^{212} \mathrm{~Pb}$, the dominant contributor of the ${ }^{220} \mathrm{Rn}$ decay products.

C Estimated from Fig. 3 in Schery (1985).

d Estimated from PAEC (44 $\mathrm{nJ} / \mathrm{m} 3)$ assuming an equilibrium factor of $0.4-0.5$. 

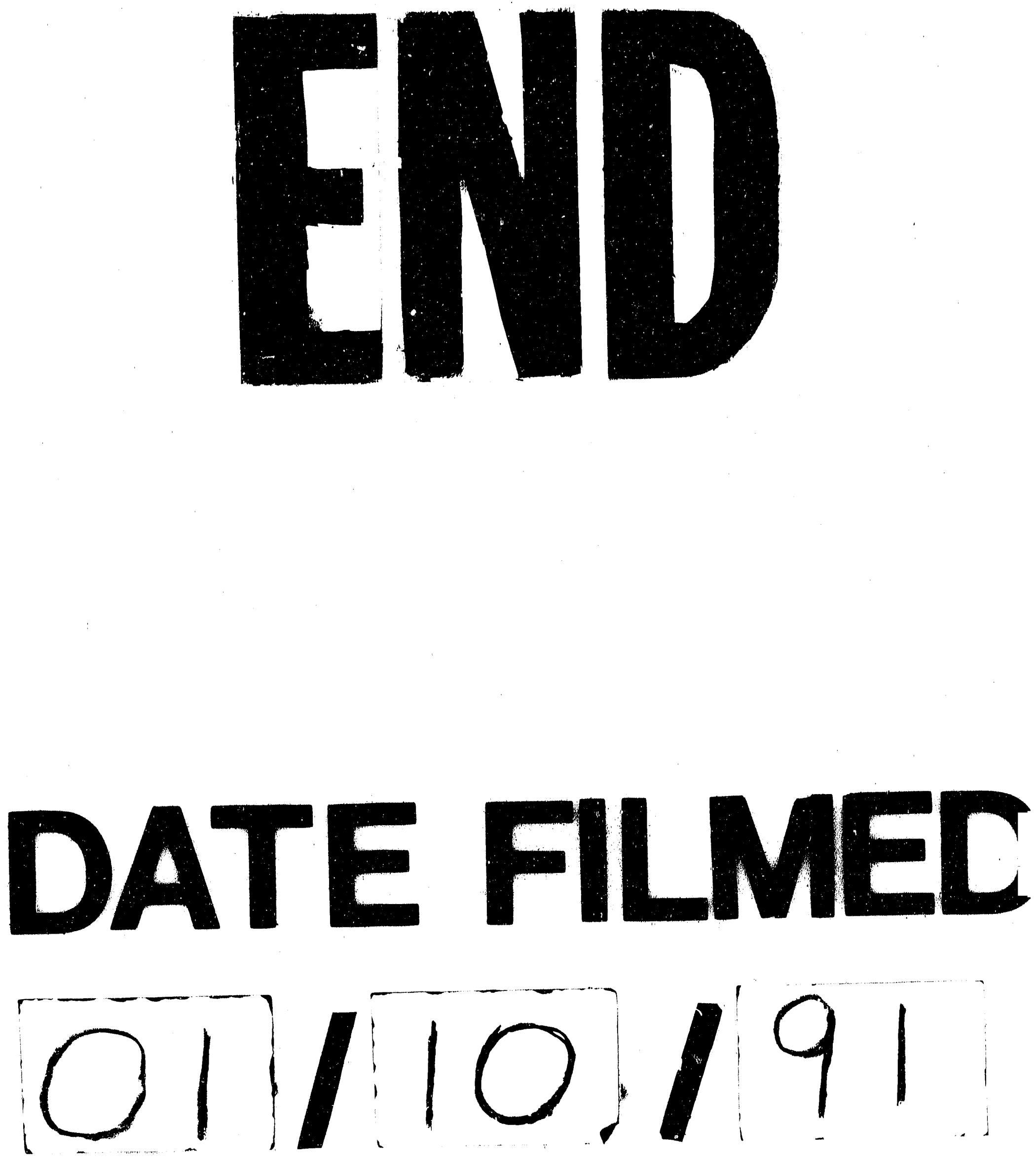
